


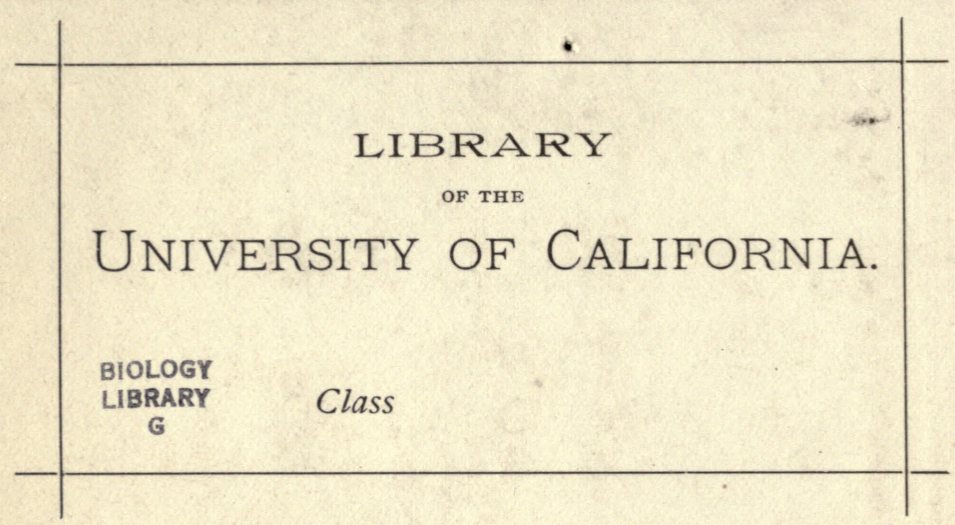



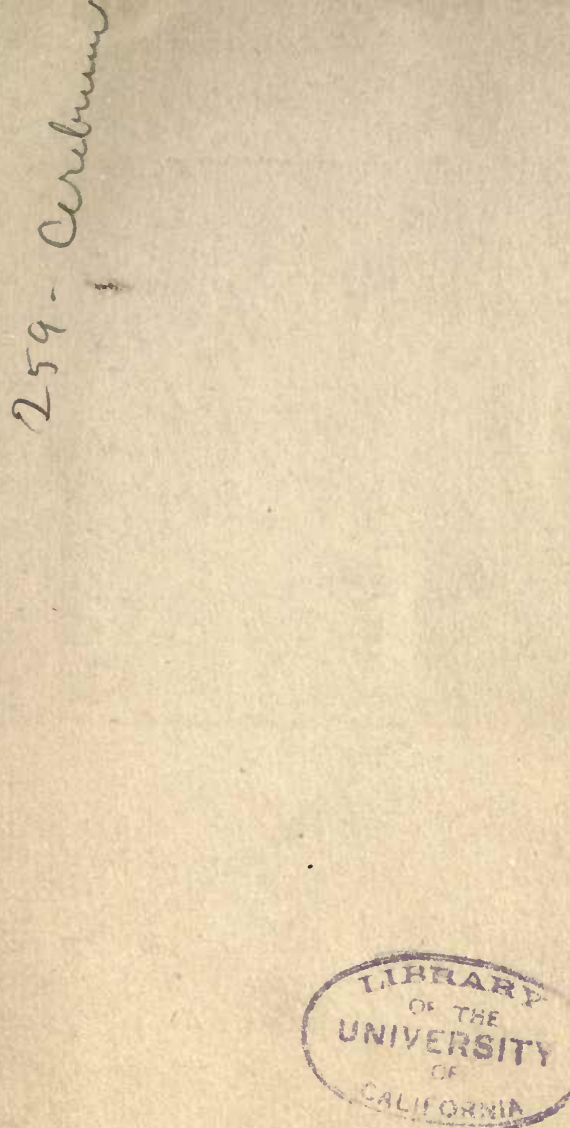
1 



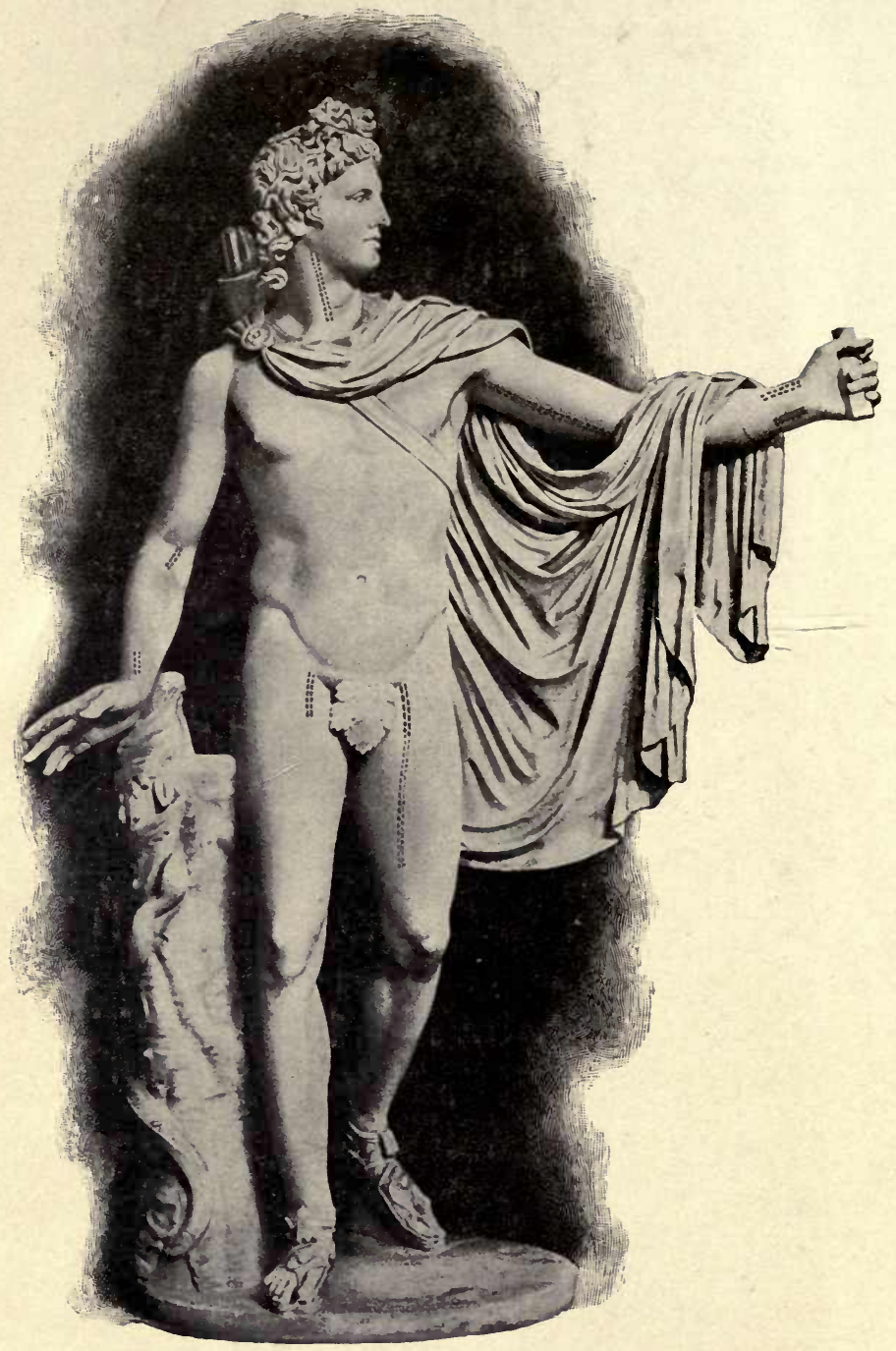

Exposed places of main arteries. 


\title{
PHYSIOLOGY
}

\section{EXPERIMENTAL AND DESCRIPTIVE}

\author{
BY
}

\section{BUEL P. COLTON, A.M.}

Author of "Practical Zoölogy," and Professor of Naturál Sclence in the Illinois State Normal University.

"What a piece of work is a man! how noble in reason! how infinite in faculty ! in form and moving, how express and admirable! in action, how like an angel! in apprehension, how like a god! the beauty of the world! the paragon of animals!"

BOSTON, U.S.A.

D. C. HEATH \& CO., PUBLISHERS 


\section{COLTON'S PHYSIOLOGIES}

PHYSIOLOGY : Experimental and Descriptive

For Normal Schouls and Colleges. 440 pages. Illustrated in colors.

PHYSIOLOGY : Briefer Course • . . . . $\quad .90$

For High Schools. 399 pages. Illustrated in colors.

PRACTICAL PHYSIOLOGY . . . . . . . 60

Laboratory Work in Physiology. I68 pages. Illustrated.

PHYSIOLOGY : Practical and Descriptive . . . 1.40

This consists of the Briefer Course and the Practical Physiology bound in one volume.

ELEMENTARY PHYSIOLOGY AND HYGIENE . . .60

For Grammar Schools. 320 pages. Illustrated.

GOOD HEALTH FOR GIRLS AND BOYS • • . .45

An Introduction to the Elementary Physiology, for Intermediate Grades, by Bertha M. Brown. I64 pages. Illustrated.

D. C. HEATH \& CO., Publishers, Boston, New York, Chicago

BY BUEL P. COL'TON. 


\section{PREFACE.}

We have all laughed at the ridiculous answers given to examination questions in physiology. But such absurd statements ought to arouse something more than laughter. They should give rise to serious thought. What do they signify? That children make gross blunders? But they do not make such wild statements about things which they have seen and handled. These absurd answers are a sad comment on the method of teaching. They point not to stupidity in the child, but to shortcoming in instruction.

Many years ago the writer became convinced of the utter futility of mere bcok-work in teaching physiology; he sought all available means of making the subject clear and full of meaning to his pupils. These illustrations, experiments, and dissections have multiplied; in the hope that what has been so useful to him may also prove helpful to others, the author presents them in this form to his fellow-teachers.

The whole of the manuscript has been critically read by Dr. G. W. Fitz of Harvard University. The manuscript has also been read by M. F. Arey, State Normal School, Cedar Falls, Iowa; E. R. Boyer, Hyde Park High School, Chicago, Ill.; H. Garman, State Agricultural College, Lexington, Ky.; N. A. Harvey, Normal School, West Superior, Wis.; W. S. Jackman, Chicago Normal School; A. J. McClatchie, Throop 
Polytechnic Institute, Pasadena, Cal. ; A. P. Ohlmacher, Medical Department, University of Wooster, Cleveland, Ohio ; and other well-known teachers of high standing.

The manuscript pertaining to the eye has been corrected by the well-known oculist, Dr. Casey A. Wood of Chicago, and that on the ear by the specialist, Dr. T. Melville Hardie.

To these the author wishes to express his sincere appreciation of their valuable assistance, and also to the following, who have read the proof, and whose criticisms and suggestions have been exceedingly helpful: A. C. Boyden, Bridgewater, Mass. ; Dr. C. F. Hodge, Clark University, Worcester, Mass.; Dr. Frieda E. Lippert, New York; Dr. L. B. Wilson, University of Minnesota, Minneapolis, Minn.; Dr. Robert H. Wolcott. University of Nebraska, Lincoln, Neb.; W. E. Wilson, State Normal, School, Providence, R.I.; Dr. Adolf Meyer, Clark University, Worcester, Mass. ; W. H. Chandler, State Inspector of High Schools, Madison, Wis.; Dr. H. B. Ward, University of Nebraska, Lincoln, Neb. ; J. E. McGi]vrey, Inspector of High Schools for the University of Illinois, Champaign, Ill.; George B. Aiton, Inspector of Higb Schools for the State of Minnesota, Minneapolis, Minn.; J. W. Crabtree, Inspector of High Schools for the University of Nebraska, Lincoln, Neb.

With this help it may be expected that the book will be fairly free from errors, yet for such as may be found the author alone is responsible.

The author acknowledges his dependence on the larger standard anatomies and physiologies, especially the Practical Physiology by Foster and Langley, the Practical Physiology 
of Stirling, and Wilder and Gage's Anatomical Technology; many hints have also been gathered from the various elementary physiologies, not only in the subject-matter, but also in the illustrations.

Thanks are due the following publishers for permission to make extracts from their books on the subject of Alcohol: D. Appleton \& Co., P. Blakiston, Son \& Co., F. A. Davis Co., Henry Holt \& Co., J. B. Lippincott Co., Longmans, Green \& Co., Macmillan \& Co., and W. B. Saunders. Especial thanks are due Mrs. Mary H. A bel for permission to use the extended extracts from the Lomb Prize Essay on Practical Sanitary and Economic Cooking.

All of the illustrations, with four exceptions, have been engraved expressly for this work. In all cases the label is placed as near as possible to the thing labeled. The author believes most strongly in this style of labeling, as he has learned that young students do not find the connection between the letters or figures of the ordinary illustration and the explanation below (usually in fine print). Diagrams have proved very useful, since many of the anatomical plates present so many details that the young student is bewildered, and does not seem able to distinguish between the important - and the unimportant features. In most cases the dorsal view is presented, so that the right and left of the organs are at the right and left of the reader. The ventral view, like a mirror, reverses right and left.

B. P. C.

NormaL, ILL., December, 1897. 



\section{TO THE TEACHER.}

The Equipment. - There should be a well-lighted room, with tables two feet wide and four and a half feet long, for two students to work together. The tables should be twentynine inches high, and provided with two drawers.

There should be an ample supply of water, soap, and towels, so that the pupils may wash their hands at the close of the work. It is very desirable to have warm water after dissecting, and a small gasoline stcve will heat water enough for several large classes. If there is no convenient sink, a long trough, made of tin or galvanized iron and stiffened by a board casing, will serve very well.

The teacher should take the utmost pains to have everything connected with the dissecting work as neat as possible. Remove all superfluous parts and scraps as soon as possible. Many of the dissections may be made on large sheets of brown paper, such as are used in the meat market. Each student should be provided with a dissecting set, which ought to include at least a scalpel, scissors, forceps, and blowpipe ; a cartilage knife is also desirable for the rough work, to save the edge of the scalpel. A more convenient form of blowpipe is made by inserting a foot of rubber tubing between the mouthpiece and the tip, as this is easier to manage, and if the student is working alone he can see what he is doing. $\mathbf{A}$ set of anatomical models is almost indispensable for good work, but these cannot take the place of dissection. Such models can be obtained of 
almost any of the school-supply firms or dealers in scientific apparatus.

Very good microscopes can be bought for twenty-five dollars and up, and no teacher should think of teaching physiology without at least one microscope.

Reference Books for the Teacher. - The teacher should have at hand for constant use one or more of each of the following sets of books :-

Avatomes. - Quain, Gray, Morris, Heitzmann.

Physiologies. - American Text-Book of Physiology, Howell; Text-Book of Physiology, Foster; Text-Book of Human Physiology, Landois and Stirling; Text-Book of Physiology, M'Kendrick; The Human Body, Martin; Human Physiology, Waller; Animal Physiology, Mills; Comparative Physiology, Mills; Hand-Book of Physiology, Kirke; TextBook of Chemical Physiology, Halliburton.

Practical Physiologies and Dissecting Guides. - Practical Physiology, Foster and Langley; Practical Physiology, Stirling; Anatomical Technology, Wilder and Gage; Physiology Practicums, Wilder; Dissection of the Dog, Howell ; A Laboratory Guide in Physiology, Hall ; Dissection of the Cat, Gorham and Tower; Zoötomy, Parker.

Works on Hygiene. - Treatise on Hygiene (2 vols.) Stevenson and Murphy; Text-Book of Hygiene, Rohé; Practical Hygiene, Coplin and Bevan; Ventilation and Heating, Billings; Practical Dietetics, Thompson; Care of the Sick, Billroth; Hand-Book of Nursing, New Haven TrainingSchool for Nurses; Text-Book of Nursing, Clara WeeksShaw; Nursing: its Principles and Practice, Isabel Adams Hampton.

The Use of the Microscope. - Practical Methods in Microscopy, Clark; The Microscope and Microscopical Methods, Gage; The Microtomist's Vade-Mecum, Lee. 
Dictionaries. - For the definition and pronunciation of technical terms consult The Century Dictionary, or Gould's Illustrated Dictionary of Medicine.

Methods of Instruction. - What we wish to have the pupil learn is the natural action of each organ, that he may understand how to keep it in good working order. Now a very common way of looking at the matter is as follows : to understand the action we must first know the structure. So the anatomy is first studied. But the child has little natural interest in anatomy, and, in fact, he needs to know comparatively little of it to understand the working of many of the organs. If we can rouse in his mind the question, "What work has this part to do, and how does it do that work?" we shall have a good starting-point, especially if we have really created in his mind a desire to get the true answer to the question. An older student may learn a good deal of anatomy patiently keeping in mind that it is the function of the organ he is seeking after all, but the younger mind has not this long foresight and patience. So, if we fail to interest him at the start, we are likely to fail in getting him to do the work in the proper way.

This method of presenting a series of problems for the student to work out under the guidance of the teacher is almost sure, with a teacher of any skill whatsoever, to give good results. Let us suppose the question to have been roused in the child's mind, "How do I raise my forearm, as in taking a mouthful of food?" we may proceed as follows:-

1. Observation of the body. Ask each pupil to feel the front of his upper arm. He observes certain changes there. (See directions in Chapter II.)

2. Dissection. A leg of a rabbit, or a sheep shank, may be brought into the class-room, and the form and general relations of a muscle seen. 
3. If a good model of the arm can be obtained, it wiil prove a fair substitute for the muscle of some other animal. show muscle action use a frog.)

4. In the absence of a muscle, or a model of a muscle, a drawing may be used. This is good as a review, especially as the parts, properly labeled, are all before the eye at one time. The teacher should always keep a supply of colored crayons; as color is a great aid, especially where there are several parts to be shown having different colors. Good physiological charts are very desirable, but often a rude drawing made by the teacher will serve a better purpose. The completed drawing, as shown in the chart, especially where many parts are shown, is apt to be confusing. Whereas, if the teacher makes the drawing, bringing out one point at a time, and labeling it as he draws it, the object seems to grow under his hand, and there is none of the confusion that comes when the whole thing is thrust upon the sight and mind at once.

5. Mechanical contrivances aid the teacher. The bones of the arm may be connected by rubber to imitate the action of the muscle. (See directions in Chapter II.)

6. The microscope may be used to show the minute structure of muscles.

7. The chemistry of many of the actions of the body may often be illustrated by very simple experiments.

The teacher and the pupil should work together to find the answer to the questions raised, to solve problems which the skillful teacher will make to appear to present themselves.

The above are some of the most helpful modes of procedure. In some cases one mode of procedure may prove more helpful, in another case another way.

So far as possible get the pupil to thinking, "How can I find out about this?" Probably some pupil in the class will suggest a good way of trying to find the answer. In this way 
the pupils are made to feel that they are doing the work. 'The wise teacher will generally endeavor to keep himself in the background, like a magnet under a paper making the particles of iron filings above take on definite arrangement, wholly unconscious of any power acting upon them.

The study of the functions of the organs is the keynote of interest in the study of all living things; and the divorcing of anatomy from physiology is proving bad for students in higher grades, and infinitely bad for elementary students. The young student will learn all the anatomy he needs to know in getting the answers to the questions about the functions of the different organs. We must take into account the psychological principle that when the mind is roused, where there is interest, there ideas may gain lodgment, and take root and grow. Of course we may continue to present the dry facts, or even continue to require the pupils to memorize the statements of a bcok, but this is not learning the actions of the body; it is merely "studying physiology" (to pass an examination), and will always furnish the absurd statements above referred to, - merely a jumble of words.

Of course the teacher, or some of the more advanced pupils, will prepare the more difficult dissections. If possible save the best dissections in alcohol. They will many times serve when fresh material cannot be obtained, and often in review when some point needs to be brought up anew.

If the physiology comes in the winter term the teacher should lay in a supply of frogs in the fall for demonstrating muscle action, the action of the nerves, reflex action of the spinal cord, circulation of the blood, etc. It is almost impossible to dispense with the frog, if these points are really to be made clear. The frogs may be easily kept in a box in a cellar, pouring a little water over them every day or two. They will not need food, for at this time of the year they are naturally 
dormant. The teacher should plan his work long beforehand, that he may have plenty of time to procure needed material.

Notes and Drawings. - The student should make careful notes of all his work in a note-book devoted to this subject. These notes should be accompanied by drawings of the dissections and copies of diagrams placed on the blackboard by the teacher, and of charts shown in the class-room. These drawings should be made in color, using water colors, where it will make the matter more clear, as in the subject of the circulation of the blood.

All the books named at the ends of the chapters should be in the school library, and each pupil should be required to read at least one in each subject. The anthor has required a review. of the books read to be placed in the note-book.

It is not necessary that all the pupils should be reading on the same subject at the same time, and in most schools it would be impossible to furnish enough duplicate copies to enable them to do so. Have the reading commence at the beginning of the term, and require each to read a book every week or two, according to a carefully made plan.

One convenient form of keeping the notes is in the style of a diary. The work of each day is entered under its date, whether this work be of practical work or of recitation.

Reform in Nomenclature. - The expressions "up" and "down," "front" and "back," are confusing when horizontalbodied animals, such as eats and rabbits, are used to illustrate human anatomy (and we must use such forms); we should use these terms: anterior meaning toward the head; posterior, toward the tail; dorsal, toward the region of the back; ventral, toward the region of the belly ; lateral, toward either side; proximal, toward the central axis of the body; distal, away from the central axis of the body. Right and left refer to the right and left of the animal and not of the student. 
The very common term "blood vessel" is exceedingly inappropriate. It suggests a receptacle, not a tube, as it really is. Especially with children we ought to employ terms that are significant, instead of terms that are either meaningless or actually misleading. So the term "alimentary canal," while less objectionable, is not so appropriate as digestive tube or food tube. These reforms are especially desirable when we introduce the study of the human body into the lower grades of school work. If the terms used are full of meaning they will help to fix the idea, instead of calling away the attention in effort of memory to keep its grip on a new word. A flood of new and meaningless terms tends to discourage the child, and to deaden his interest. Foster (one of the very foremost of physiologists) sets the good example of substituting spinal bulb for the long and cumbersome term medulla oblongata. Thoracic should be substituted for dorsal in such cases as "dorsal vertebræ;" we ought to say thoracic vertebræ. Precaval and postcaval veins we should say, instead of anterior vena cava, posterior vena cava.

Relative Value of Topics. - The relative value of the different topics ought to be considered. For instance, most of the elementary text-books of physiology pay an undue amount of attention to the bones, when we consider to what a slight extent a knowledge of bones aids in preserving health. It would seem sufficient to have the skeleton present (or some of the separate bones, if the whole skeleton cannot be obtained), and to refer to it in connection with the study of the more important organs. The bones, for the most part, play a wholly subordinate part in the work of the body, being passive in their action, serving as levers, for protection, etc. Therefore, when the muscles and motion are studied, let the bones be introduced, to show that they act as levers in applying the force of the muscle; when the brain is 
studied, look at the brain case, etc. The bones by themselves are a pretty dry subject; and the teacher must not allow the class to lose its interest for one day even, or he may never recover that interest.

Order of Topics. - The order of topies is a most important matter in the study of physiology. We should not only try to begin with the study in such a way as to get and hold the interest ; the topies ought to be arranged in such an order that beginning with what is simple we may proceed by an easy and natural gradation to that which is more difficult, so that the earlier topics shall prepare the way for the latter. Of course the study of any part of the body throws light upon the study of every other part. Still, it is possible to make such arrangement that the topic presented first be of such a nature as best to explain itself, to stand alone, so to speak, and to leave till later the study of actions that directly depend on many others, and that cannot be explained without introducing those others.

For example, suppose we were to begin with the digestion, which is often presented as the first of the fundamental, vital processes of the action of the body (the bones and muscles, perhaps, having had a little attention).

In the study of digestion we are met by the action of muscles in masticating the food, in swallowing, and throughout the whole digestive tube. Shall we leave the explanation of muscle action, or stop and take it up?

Saliva is poured upon the food in the mouth. Shall we stop and explain the action of a gland? This involves a knowledge of circulation. To understand how the gland gets more blood at this time, out of which to make the saliva, we must understand the action of the muscles in the walls of the arteries supplying the gland. To understand the control of these muscles we must know considerable of the nervous system. And so on throughout the whole process of digestion. 
We meet difficulties at every step if we have not studied the details of circulation and nervous and muscular action.

The nerves control not only the muscles, but every activity of the body. They are the key to the whole mechanism. Why should we leave the study of their work to the very last, especially since we know that whatever is last in a book is likely to be gone over hurriedly?

Throughout the book the natural sequence of topics has been studied with care, and the relation of one part to another kept in mind.

The order adopted is the result of twenty years' experience in teaching this subject. After trying almost every possible order this one seems to offer the "least resistance" to the progress of the pupil. The effort of the teacher should be so to arrange the topics that the easiest should come first and thus readily lead the way to the next, as the first step of the stair makes it more easy to reach the step above it.

There is no royal road to learning; it is up-hill work at best; but the teacher has no right to try to lead the pupils up the precipitous side of a mountain if there is a side where the slope is gradual, where a relatively easy and natural path may be made.

"Instruct us where the mountain low declines,

So that attempt to mount it be not vain.

For who knows most, him loss of time most grieves."

DANTE. 



\section{CONTENTS.}

CHAPTER I.

INTRODUCTION

CHAPTER II.

Motion

Experiments with the Muscles in our own Bodies. - Muscle and Nerve. - The Action of Muscle. - Structure of Muscle. - Importance of Muscles. - Laws of Muscle Action. - Symmetrical Development of Muscles. - Relation of the Muscles and the Bones. Levers. - Study of one of the Long Bones. - Joints. - Locomotion.

\section{CHAPTER III.}

'The General Functions of the Nervous System. - SensaTION AND MOTION . . . . . . . . . . .

The Brain and Spinal Cord of the Cat or Rabbit. - Structure of Nerves and Nerve Fibers. - Cross-section of the Spinal Cord. Reflex Action of the Spinal Cord of the Frog. - Nerve Roots and their Functions. - Functions of the Spinal Cord. - Voluntary Interference with Reflex Actions. - The Nature of a Nervous Impulse. - Harmony in Muscle Action. - Dependence of Nerves and Muscles.

\section{CHAPTER IV.}

Cimculation of the Blood . . . . . . . . . . . .

The Blood and its Work. - The Heart-beat and the Pulse. A Drop of Frog's Blood. - The Circulation of Blood in the Web of a Frog's Foot. - Heart and Lungs of a Pig, Sheep, or Calf. - Demonstration of the Action of the Heart. - Reason for Injecting the Arteries. - The Distribution of the Arteries and Veins in a Cat or Rabbit. - The Action of the Frog's Heart. - The Rate of the Heart-beat. - The Action of the Heart. - The Beat of the Heart. The Sounds of the Heart. - Action of the Large Arteries. - Ac- 
tion of the Medium-sized Arteries. - The Blood-flow in the Capillaries. - The Veins. - The Valves in the Veins. - Effect of Pressure on the Veins. - Rate of Blood-flow in the Arteries, Capillaries, and Veins. - Nourishment of the Walls of the Heart and Blood-tubes. - Dissection of the Sympathetic Nervous System. Control of the Heart and Blood-tubes by the Nervous System.Effects of Alcohol on the Circulation. - The Blood. - The Coagulation of Blood. - The Lymph. - The Spleen. - Massage.

CHAPTER V. Respiration
The Organs of Respiration.-The Movements of Respiration.
- Forces of Respiration. - Capacity of the Lungs. - Hygiene of Breathing. - Respiratory Sounds. - The Control of Respiration. The Chemistry of Respiration. - The Production of Heat and Motion in the Body. - The Two Breaths. - Heating and Ventilation. - Breathing through the Mouth.-Dead Dust.-Live Dust. . Bacteria.

CHAPTER VI.

EXCRETION

The Skin and its Functions. - The Structure of the Skin.The Sweat Glands. - Composition and Amount of Sweat. - Regulation of the Temperature of the Body by the Skin. - Distribution of Heat in the Body. - Regulation of the Production of Heat in the Body. - Regulation of Bodily Heat by Food and Clothing. - Sunshine.-The Kidneys. - Dissection of the Kidney. - Microscopic Structure of the Kidney.

\section{CHAPTER VII.}

\section{Digestion}

Foods and Foodstuffs. - The Proteids. - Proteid-containing Foods. - Cheese. - Fats. - The Carbohydrates and Carbohydratecontaining Foods. - Salts. - Necessity of a Mixed Diet. - Tea and Coffee.-Alcohol. - Tobacco. - The Digestive System.-Organs of the Abdominal Cavity. - The Organs of Digestion. - The Teeth. - The Kinds of Teeth and their Arrangement. - The Care of the Teeth. - Dissection of the Head of the Rabbit. - Experiments with the Digestive Liquids. - The Bad Effects of Imperfect Mastication. - The Process of Swallowing. - The Stomach. - The Intestine. - Absorption. - The Lacteals and Lymphatics. - Constipation. - Hygiene of Digestion.-A Good Dinner. - Taking Cold.-Bathing. - Exercise.-Forms of Exercise.-Boxing. - 
Ledger Account of the Body and its Organs. - A Living Eddy. Fat. - Glycogen. - Nutrition. - The Indestructibility of Matter. - The Indestructibility of Force.

\section{CHAPTER VIII.}

The Brain . . . . . . . . . . . . . . . . 253

Directions for Preparing the Brain of the Cat or Rabbit. Study of the Brain.- The Cranial Nerves. - The Functions of the Cranial Nerves. - The Cerebrum and its Functions. - Location of Brain Functions. - Functions of the Cerebellum. - The Spinal Bulb. - Blood Supply of the Brain. - Brain Work and Brain Rest. - Nerve Stimuli. - The Nature of Sensation. - The Relative Nature of Sensations. - Effects of Alcohol on the Nervous System.Moral Deterioration Produced by Alcohol. - Narcotics. - Opium. - Hashish. - Habits.

\section{CHAPTER IX.}

\section{The Special Senses}

General Sensations and the Special Senses. - What We Learn by Touching Objects. - Nerve-endings in the Skin. - The Sense of Touch. - The Pressure Sense. - The Localization of Touch Sensations. - Illusions of Touch. - The Temperature Sense. - The Muscular Sense. - Pain. - Hunger and Thirst. - The Sense of Taste. - The Sense of Smell. - The Sense of Sight. - The Muscles of the Eyeball. - External Parts of the Eye. - Dissection of the Eye.The Retina. - The Center of Distinct Vision. - Protection of the Eye.-Adjustment of the Lens for Seeing at Different Distances. - The Blind Spot. - Regulation of the Amount of Light Admitted into the Eye. - Color Sensations. - Color Blindness. - Stereoscopic Vision. - Duration of Impressions of Light. - The Care of the Eyes. - The Sense of Hearing. - The Equilibrium Sense. - The Care of the Ear.

CHAPTER $\mathrm{X}$.

The Volce . . . . . . . . . . . . . . . . 320

The Larynx of the Calf. - Dissection of Larynx. - Voice and Speech.

\section{CHAPTER XI.}

\section{ACCIDENTs .}

How to Stop Flow of Blood from Wounds. - Bleeding from the Nose. - Burns. - Fainting. - Broken Bones. - Sunstroke. Treatment of the Drowned. - Suffocation in Wells. - Poisons and 
PAGF

their Antidotes. - Wounds from Thorns, Rusty Nails, Bites of Cats, Dogs, etc. - Snake Bites. - The Sick-room.

CHAPTER XII.

The Skeletón . . . . . . . . . . . . . . . . . . 346

The Skeleton as a Whole.-Axial Skeleton. - Appendicular Skeleton.-Study of Vertebræ.-Microseopic Structure of Bone. - The Chemical Composition of Bone.-Hygiene of the Bones and Joints.

APPENDIX A.

Foods AND CoOKING. . . . . . . . . . . . . . . 357

APPENDIX B.

Antiseptics AND Disinfectants . . . . . . . . . . 378

APPENDIX C.

Tables of Statistics, Etc. . . . . . . . . . . . . . . 380

Glossary . • . . . . . . . . . . • • • . 389 


\section{PHYSIOLOGY,}

\section{EXPERIMENTAL AND DESCRIPTIVE.}

\section{CHAPTER I.}

\section{INTRODUCTION.}

Is it not a splendid thing to be well and strong? To be full of bounding health ? To "feel one's life in every limb" ?

Who does not desire to prolong, so far as possible, this condition characteristic of youth?

Is it not a pity that in so many persons health begins to break down in middle life? That when the mind is beginning to ripen, the body should begin to decay? Our later years ought to be our better years. Our mental strength and maturity should then enable us to give back to the world something for what it has given us; for in the earlier years the individual is constantly receiving, and making little return. But bodily strength and vigor are necessary in order that a rich, and ripe mind may exert its influence upon the world. If the days are filled with pain, if there is feebleness of body, one is likely to become a burden to the world, instead of a bearer of its burdens. Witness Holmes and Gladstone as examples of men who took excellent care of themselves and were able to continue their work in a ripe old age.

An animal, living in a state of nature, may keep well and live its natural period of life without knowing anything about the laws of health. But as students or indoor workers, many 
of us lead a sedentary life; we are not natural, but oftec highly artificial, in our mode of living. We move about but little, whereas the animal abounds in motion. We concentrate our energy upon mental effort, thus diverting a large share of our sum total of energy away from the process of nutrition. We often shut ourselves up in rooms too nearly air-tight. We eat poorly chosen and ill-prepared food. We devour it in haste, often when we are in a condition unfit for taking food. In short, we too frequently disobey the laws of Nature. Now, Nature punishes every violation of her laws. She never forgives, never forgets. Whether we trespass in ignorance or wittingly, she punishes. But while Nature punishes disobedience, she, on the other hand, gives rewards for obedience to her laws. Health and happiness are her rewards. Are they not sufficient? We may abuse her kindness; but she never sleeps, never fails to notice. We may, for a time, think we are to escape; but we find the punishment, though delayed, perhaps the more severe.

The out-of-door worker may not suffer so much from ignorance in these matters. From the character of his occupation, he to a certain extent is obliged to obey Nature. He gets enough fresh air. His bodily exertion generally brings a hearty appetite, vigorous digestion, active circulation of the blood. Still, he would greatly profit by knowing something as to the nature of his food, its wholesomeness or unwholesomeness. The fact that he gets along fairly well is no proof that he always does the best thing. His natural mode of life may keep him in tolerably good condition in spite of his violation of certain laws; but he could undoubtedly learn much as to economy in the purchase, preparation, and proper combination of foods.

But with those of us who strive to lead "the intellectual life," the price of our culture is high. We often do so at the 
expense of animal vigor. Unless we give care to the matter of exercise, the blood circulates too slowly, the appetite and digestion are likely to become somewhat dull, and the whole body suffers.

In regard to any machine of man's invention we know that we must keep it in good running order if we would have it do good work, or last long. We must keep a machine clean, well oiled, and not work it too hard. Are not our bodies worth equal care? If some part of a machine is broken, we may replace it at moderate expense; but none of the vital organs can be replaced. We may get a new mainspring for a watch, but we cannot get a new stomach or lungs.

In the deacon's "One-Hoss Shay," all the parts were equal in strength and durability, so that it went to pieces -

"All at once, and nothing first, Just as bubbles do when they burst."

In our bodies, usually some organ is weaker than the others. If this be true of one of the more important organs, - the heart, liver, stomach, lungs, kidneys, - then the whole organism must stop, because no substitution can be made. Since most of us inherit weakness of some organ, it behooves us to find out.what forms of disease have afflicted our ancestors, and then to guard carefully the weakest part. A person may be strong in all the organs but one, and yet break down; whereas a person apparently feeble, yet well balanced, may live longer, and have much better health. In fact, great strength is often a source of great weakness. If one has powerful muscles, and overworks them, he may overstrain the heart, and consequently break down.

The student ought to have his hours for exercise just as regularly as his hours for study; and it is a matter of congratulation that so many of our educational institutions insist 
on physical training as much as on any other part of the curriculum.

Left to himself, many a student becomes absorbed in his study, and neglects his body. Others may run to the opposite extreme. All should be under proper direction and control. 'There are so many cold and stormy days in this climate that the gymnasium is a necessity for educational institutions. It is a fine thing that athletic sports of all kinds are now much in vogue. They may be overdone, but time will right this. It is to be hoped that good health will never again be out of fashion for young women as well as for young men.

The student who "hasn't time" to play tennis, or ball, or something of the sort, is likely to lose the time at the other end of his life. The student or other indoor man who cannot take his hour a day for recreation is often extremely short-sighted. One-tenth of his time thus employed may double the length of his life, and make it very much more enjoyable.

The student who cannot take time to stop study for exercise is about as wise as the woodsman who has not time to stop and sharpen his ax. He persists in trying to learn his lesson with a dull brain. If he can be induced to sharpen his brain by exercise, he will find how much time he can save by "wasting" a little in play.

Aside from the above considerations, the human body is worthy of study for its own sake. Viewed simply as a mechanism, it is wonderful. Each organ is so well adapted to its work, and all the organs work so harmoniously through their connection and control by the nervous system, that we never cease to admire. We admire a doll, or other toy, so ingeniously constructed that it can move its eyes, or walk a short time after being wound up. We admire a mechanism that 
can rudely imitate the human voice. But this live mechanism, which is self-winding, self-regulating, self-repairing, selfdirecting, amazes us.

We take up the study of the human body mainly that we may learn how to preserve health; the science of health is Hygiene.

In order to keep the various organs in good order we must know what their natural work is, and how they do it; the science of the action of the body and its parts is Physiology. The work, or action, of each part, or Organ, is its Function.

In order to understand the working of each organ it is usually necessary to know something of its construction; the science of structure is Anatomy. But we do not need to go far into anatomy to get a fair knowledge of the manner in which our organs do their work. The surgeon, of course, must be able to locate accurately the various blood tubes, nerves, muscles, etc. All we shall need to know of anatomy is the general structure of the body and of some of the more important organs, such as the heart and lungs, the larynx, the eye, etc. It is fortunate for us that these organs in the sheep, pig, and cow are so nearly like our own that they serve admirably in enabling us to understand ourselves.

We know that any organ is composed of several different kinds of material. For instance, in a slice across a ham we see the skin on the outside, then fat and lean and bone. These "primary building materials" of the body we call Tissues. These different tissues we shall study as we come to them.

The whole body is made up of small particles called Cells, comparable to the bricks in a house. These cells are of various shapes in the different tissues. With the blade of a very dull knife, or the handle of a scalpel, gently scrape the inside of the cheek. Place a little of the white scraping on a slide 
in a drop of water, cover with a coverslip, and examine under a quarter-inch objective. Many cells will be seen, some of them showing nuclei. Compare these cells with the accompanying figure.

In the active tissues the cells are alive, and each cell may be considered as like the Ameba, a little mass of living jelly-like substance called Protoplasm. Within this is a small, rounded part called the Nucleus. Most of the cells of the body differ from the Ameba in having a distinct outer covering or Cell-wall. A grape serves very well to show what a cell is like. Now, the whole body is built up chiefly of such little cells, few of them large enough to be seen by the naked eye. Although the cells are closely

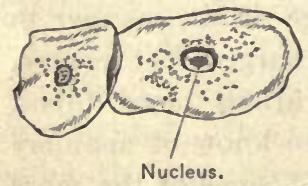

Fig. 1. Epithelial Cells from the Inside of the Cheek. packed together, each cell leads, in one sense, an independent life. But all work together to maintain the life of the body as a whole. The cell in the body is like the individual in a community. Each lives primarily for itself, yet all work together for the good of the whole. Each has its own kind of work to do, as have the individuals of the community.

The Physiological Division of Labor. - We are aware of the advantages of division of labor in a community. If each person learns to do one thing well, all work economically together for the common good, time is saved, and better goods of all sorts are produced. As a rule, civilization is at a low ebb where every individual is a "jack-of-all-trades" aud master of none. In the body there is a division of labor similar to that of a community. Each of the different organs has its own work to do, and all work together for the common welfare. Each of the tissues is composed of cells characteristic of that tissue. The cells of one tissue have certain properties 
and peculiarities of form differing from the form and properties of the cells of any other tissue. The general structure of all cells is essentially the same, just as all men are alike in their plan of structure. But one man learns to do one kind of work, and sometimes can hardly do anything else. Often the nature of his work can be judged from his appearance. With the cells, while they all have the same essential structure, and have certain properties in common, each has some one kind of work that it can do well, and to which work it devotes itself. The nerve cells receive impressions from the outer world, carry nervous impulses, and control the various activities of the body. The muscle cells have as their one work the production of motion. All the cells must take food for themselves and grow. Each has a birth, life, and death, as each individual in a community of men; and as the community endures, while the individual members who compose it are continually changing, so, in the body, while the form remains about the same from year to year, the cells are continually changing, some dying, and others taking their places.

In an animal made of a single cell, like the Ameba, the one cell must do everything for itself. The higher animals all begin their individual life as an egg, which is, in fact, a single minute cell. This grows and divides, forming two cells. By repeated division there accumulates a mass of cells. These take on the arrangement peculiar to the kind of animal from which the egg came. But as the cells increase in number one group of cells takes up one part of the work of the body, other cells another part of the work, and so on. An individual living alone (Robinson Crusoe) had to do everything for himself. Just as soon as this "community of one" became a community of two by the accession of Friday, there sprang up a division of labor.

In studying history (sociology) we have to deal with. the 
individual, the community, the state, and the nation. The individual is a cell, the community is a tissue, the state is an organ, and the nation is one body.

Let us proceed to study the nature of the individual cell, and the combined actions of these individuals in that community called the human body. 


\section{CHAP'TER Il.}

\section{MOTION.}

Motion is the most manifest sign of life. While we are sitting still, as we say, there are frequent slight motions of the head, body, and limbs. Even during sleep the movements of breathing may be seen; the hand laid upon the chest may feel the beating of the heart, and the finger detect the pulse in a number of places.

When an animal shows no motion whatever we conclude that it is no longer alive. We must move to get our food, or at least to eat and digest it. Motion is necessary for breathing, for circulating the blood, for getting rid of wastes. We often move to avoid injury.

Motion is necessary for seeing: we must turn the face toward the object and open the eyelids; we move the eyeballs; within the eye are motions to regulate the amount of light admitted, and to adapt the eye for seeing at different distances.

In feeling, we put forth the hand to touch the object. In tasting, we place the object upon the tongue, or touch the tongue to the object. In smelling, we sniff; and sniffing is a respiratory motion.

In hearing, we usually turn the head, and there is motion in the ear to adjust the parts to accurately distinguish sounds.

In speech there is motion. There is motion in facial expression; or we may communicate by signs, as in writing, or talking with the hands. 
In all these instances, both in the reception of knowledge through any of the senses and of the expression of knowledge through any kind of language, spoken or written, or facial expression, signs, or gestures, some motion is absolutely necessary.

How are all these motions, so essential to every process of life - how are they produced?

Experiments with the Muscles in our own Bodies. -

1. Clasp the front of the right upper arm; draw up the forearm strongly and as far as possible. Note what changes are felt in the Biceps muscle.

2. Repeat the experiment, and with the thumb and finger feel the cord, or tendon, at the lower end of the muscle, just within the angle of the elbow.

3. Place a weight in the hand, and repeat the act, noting the condition of the muscle during the experiment; also note the condition of the tendon.

4. Span the muscle, placing the tips of the fingers in the angle of the elbow, and the tip of the thumb as far as you can up the arm; again bend the arm. What change in the muscle does this show?

5. Any muscle that bends a limb, as does the biceps, is called a Flexor muscle.

6. Clasp the back of the right upper arm; forcibly straighten the arm. The muscle lying along the back of the arm is the Triceps muscle. It is called an Extensor muscle because it extends, or straightens, the arm.

7. Clasp the upper side of the right forearm near the elbow; clench the right hand quickly and forcibly; repeat rapidly.

8. Notice the thick mass of muscle at the base of the thumb; pinch the forefinger and thumb strongly together. What changes can be seen and felt? 
9. Place the hand on the outside of the shoulder; raise the arm to a horizontal position; repeat with a weight in the hand.

10. Stand erect with the heels close to each other, but not quite touching; let the arms hang freely by the sides; rise on tiptoes, without moving otherwise; repeat twenty times.

11. Place the tips of the fingers on the angles of the lower jaw; shut the teeth firmly on a piece of rubber, and note the bulging of the Masseter muscles.

12. Press the fingers on the temples; again shut the jaw firmly, and feel the action of the Temporal muscles.

13. Make a narrow band of paper that will snugly fit the forearm when the hand is open; now clench the fist strongly.

14. With a tape-measure get the circumference of the up-

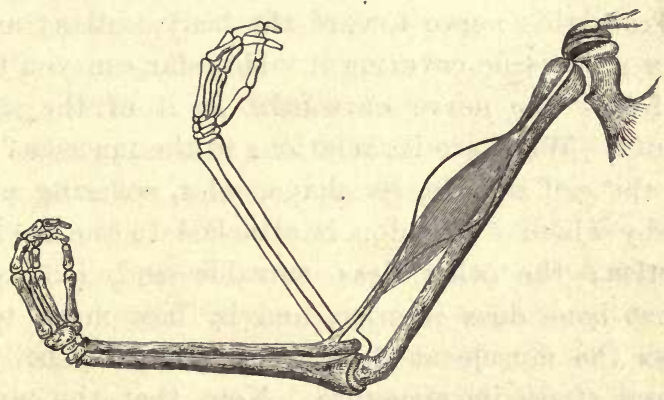

Fig. 2. The Shortening and Thickening of the Biceps Muscle in raising the Forearm.

per arm when the arm hangs free; again when the forearm is strongly flexed.

15. In the same way measure the forearm when the hand is open, and when the hand is clenched.

By these experiments we learn that when a muscle works it becomes shorter, thicker, and harder. 
In order to understand the subject better, let us look at the muscles of a rabbit's leg. 'The dressed rabbits found in the markets will serve very well, but it is better not to have the skin removed until just before studying the muscle. 'Two students may conveniently work together.

Muscle and Nerve. - In the hind limb of the rabbit observe the heel cord, or Achilles' tendon, passing upward from the heel along the back of the leg. The tendon is the termination of the calf muscle, which lies on the back of the shinbone. Trace this muscle toward the body, and note that it passes between two large, flat muscles, one on the inner, the other on the outer, back part of the thigh. Separate these two flat muscles, using mainly the handle of the scalpel. Remove any fat that may be in the way. Deeply imbedded between these muscles is a white cord, the Sciatic Nerve. Trace this nerve toward the body, cutting away any muscles or soft tissue covering it. How far can you trace it?

Now follow the nerve outward. Is it of the same size throughout? What are its relations to the muscles? Study carefully the calf muscle, its shape, color, covering, ends, etc. The end by which its tendon is attached to the heel-bone is the Insertion; the other, less movable end, is the 0rigin. From what bone does it arise, and by how many tendons? Cut across the muscle at its thickest part, the belly of the muscle, and study its structure. Note that the tendons at the ends of the muscle are continuous with the muscle sheath and with the partitions running through the muscle.

Pull the tendon toward the body: this straightens, or extends, the foot; the calf muscle is therefore called an Extensor muscle. With the handle of the scalpel loosen the muscle on the front of the shin-bone; prove that its action is to bend, or flex, the foot. It is a Flexor. Find its origin and insertion. 
We have now seen the muscles themselves, not covered by skin. Our muscles are very much like those of the rabbit, only larger. But if we would learn more fully the action of muscle, we need to go to another animal, - the frog.

The Action of Muscle. - Kill a frog thus : Into a fruitjar of water put a teaspoonful of ether; immerse the frog in it and cap the jar. As soon as the frog is motionless cut off its head and run a wire down the cavity of the spinal column, to destroy the spinal cord. Cut through the skin around the base of one of the thighs, and strip off the skin from the whole of the limb. Note that the muscles are of a pale color. The muscles of a frog's thigh are nearly the same in number and arrangement as in man. Examine more thoroughly the calf muscle; the end by which it is attached below is its insertion, and the upper attachment is its origin.

Sever the limb from the body at the hip joint. Separate the muscles along the outer back part of the thigh, and find the white, thread-like Sciatic Nerve. The nerve must be handled with great care; it must not be pinched or dragged. Carefully separate it from the surrounding muscles, and turn it down upon the calf muscle. Cut away all the muscles of the thigh, being careful not to touch the nerve where it runs down by the knee. Sever the heel cord below the heel, and separate the calf muscle from the rest of the leg, leaving undisturbed its attachment above; just below the knee cut away the shin-bone, with all the muscles of the leg except the calf muscle.

There should now remain the Thigh-Bone, with the Sciatic Nerve running to the Calf Musele suspended below. Fasten the thigh-bone to some support, such as a clamp or ring on a retort stand. Attach a small hook to the tendon, and suspend from it a slight weight, such as a small key.

Such a preparation is called a Nerve-Muscle Preparation. 
It should frequently be moistened with a .7 per cent solution of common salt in water, called Normal Saline Solution.

Now take a sharp pair of seissors, and snip off the shortest possible portion of the upper end of the sciatic nerve. If the muscle is closely watched at the time when the nerve is cut, it will be seen to thicken and shorten, and to lift the weight. If the muscle be held between the thumb and finger while the nerve is pinched (and the scissors are the surest pincher's) it will be felt to harden.

This experiment should be repeated, varying the weight, until it is made very clear that when the nerve is stimulated

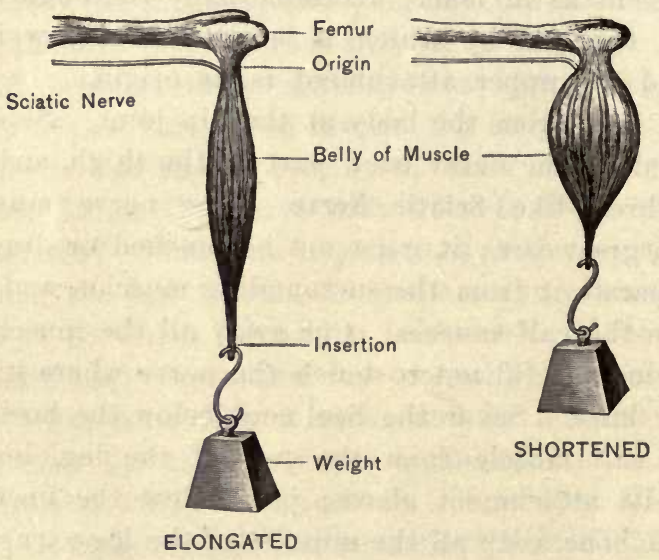

Fig. 3. Action of the Calf Muscle of the Frog, showing the Relations of the Sciatic Nerve.

the Muscle Shortens (which is the most important fact dbout the action), thickens, and grows harder.

Structure of Muscle. - Chipped beef shows well the structure of muscle. Looking at a piece closely we see 
work of white substance; this is the Connective Tissue. In the meshes is the red muscular tissue. The partitions which run all through the muscle, and the cross-sections of which we see in a piece of chipped beef, are continuous with the Muscle Sheath, and both are continuous with the tendons at the ends of the muscle. In fresh muscle the sheath and the partitions are nearly transparent, and are not easily seen. When the meat is cooked or salted the connective tissue becomes opaque and is white.

In frog's or rabbit's muscle observe the thin, trans-

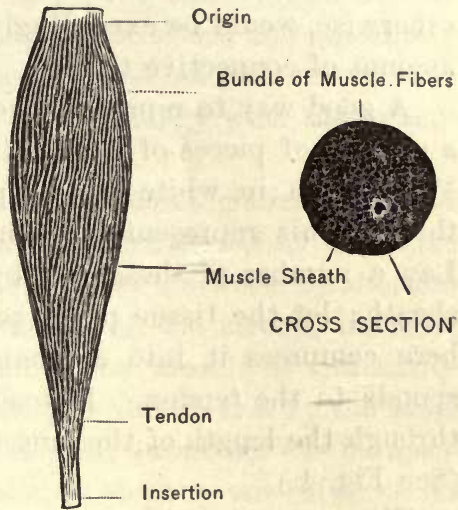

LONGITUDINAL SECTION

Fig. 4. The Structure of Muscle. parent membrane covering the muscle, the muscle sheath.

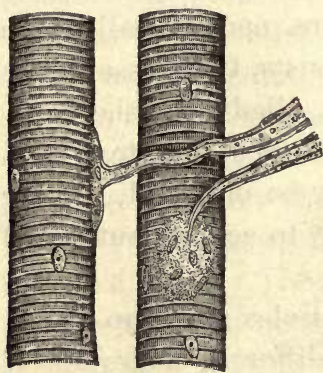

Fig. 5. Two Muscular Fibers, showing the Terminations of the Nerves.
With forceps tear away part of the muscle sheath. Tear the muscle to pieces, and note its fibrous structure. Put a shred of muscle in a drop of normal saline solution on a slide, and examine with low power of the microscope; again examine with a higher power. The cross-markings, or striations, will be seen.

Such muscle is called Striated or Striped Muscle. All of the muscles used in ordinary motions are of this kind.

In well-cooked corned-beef the connective tissue is thor- 
oughly softened, and the muscle fibers are easily separated. Thorough cooking, especially slow boiling, will soften the connective tissue, and may render palatable meat that, cooked otherwise, would be exceedingly tough on account of the large amount of connective tissue.

A good way to represent the structure of muscle is to take a number of pieces of red cord to represent the muscle fibers. Wrap each in white tissue paper a little longer than the thread; this represents the individual fiber with its sheath. Lay a number of these side by side; wrap all in a common sheath; let the tissue paper project beyond the threads, and here compress it into a compact cylinder; this last corresponds to the tendon. Instead of one long fiber continuous through the length of the muscle, there are many short fibers. (See Fig. 4.)

The connective tissue forms a framework for all the tissues of the body; and if their working cells were removed, the connective tissue would remain like the framework of a squeezed lemon, or like the skeleton of a sponge after its soft tissues are removed, and showing more or less completely the form of the part. Connective tissue, therefore, may be called the skeleton of the soft tissues. Muscle consists, then, essentially of a collection of soft, transparent tubes, filled with the semifluid muscular fibers. By scraping the surface of a steak with a dull knife the muscular substance may be obtained, leaving the connective tissue. This is a good way to get the nutritious part of beef for an invalid.

Importance of Muscles. - The muscles make up nearly half of the weight of the body. The different materials of which the body is built up are called Tissues. Thus we find muscular tissue, bony tissue, nervous tissue, etc. Now, the muscles weigh nearly as much as all the other organs of the body taken together. This fact, of itself, should lead 
us to consider the muscles of high importance. Add to this the facts above noted, that the muscles are so largely concerned in the nutrition of the body, the chief agents for its protection, essential for the reception of ideas, and absolutely indispensable for the expression of ideas, and we can see the reason for beginning the study of physiology with the examination of the muscles and their action.

Laws of Muscle Action. - The chief characteristic of muscle is its ability to shorten; incidentally, it at the same time thickens and hardens. But it does its work by shortening, pulling on the bones by means of the strong, inelastic tendons, thus producing motion. The muscle is ordinarily said to "contract;" but as it occupies almost exactly the same amount of space at all times, the word "contract" is inappropriate. A muscle may be made to shorten one-third of its length, but probably never shortens that much in the living body.

A muscle cannot be kept shortened for any great length of time. If one holds his arm out horizontally as long as possible he soon feels fatigue, later pain, and he may feel soreness in the muscle for several days. The law of muscle action is to alternate periods of rest with periods of action. In many exercises, as in walking, the limbs act alternately, one resting or recovering position while the other works.

If we consider the biceps and triceps of the arm, we see that they are compelled to act alternately if they would do effective work. They might both shorten at the same time, and are made to do so in such an attempt as that of holding the arm rigidly bent at a right angle; as, for instance, in wrestling "square hold," in which case one wishes to prevent his opponent from either pushing or pulling him. But while the two muscles act no motion is produced. When the flexor shortens the extensor lengthens, and vice versa. 
The muscles are always slightly stretched, as shown by the fact that when a cut is made into a muscle the wound gaps open; the tension of the muscle is further shown by the fact that when a bone is broken, as in the upper arm or thigh, the ends of the bones slip by each other, and the limb has to be strongly stretched to bring the ends back together. Muscles act better when slightly stretched, and probably need a slight resistant action of the opponent muscle.

Symmetrical Development of the Muscles. - The muscles of the two sides of the body are the same in number and arrangement. At birth they are probably about equal in size, weight, and strength. Most persons early become righthanded, and the greater use of the right hand and shoulder makes the muscles of this side larger and heavier. The muscles pulling on the bones slightly modify them in shape. The whole body may become noticeably unsymmetrical. Most persons step harder on one foot than on the other, as shown by the sound of the footstep, and as shown by the constant wearing of one shoe sole or heel faster than the other. This is often caused by a tight shoe, or a peg in the sole, or other irregularity, that for the time made it.painful or uncomfortable to bear the whole weight on that foot. So the habit may be formed for life. The shoes of children should be carefully examined. Better throw away a pair of shoes than make a limper for life. The majority of persons limp, though not lame.

In very many persons one shoulder is habitually carried higher than the other. Symmetrical development should be carefully sought, and any tendency to a one-sided development should, so far as possible, be avoided.

We should use the left hand more. It should not be so often "left." Surgeons and barbers are usually ambidextrous. Probably it would not pay to try to do everything equally 


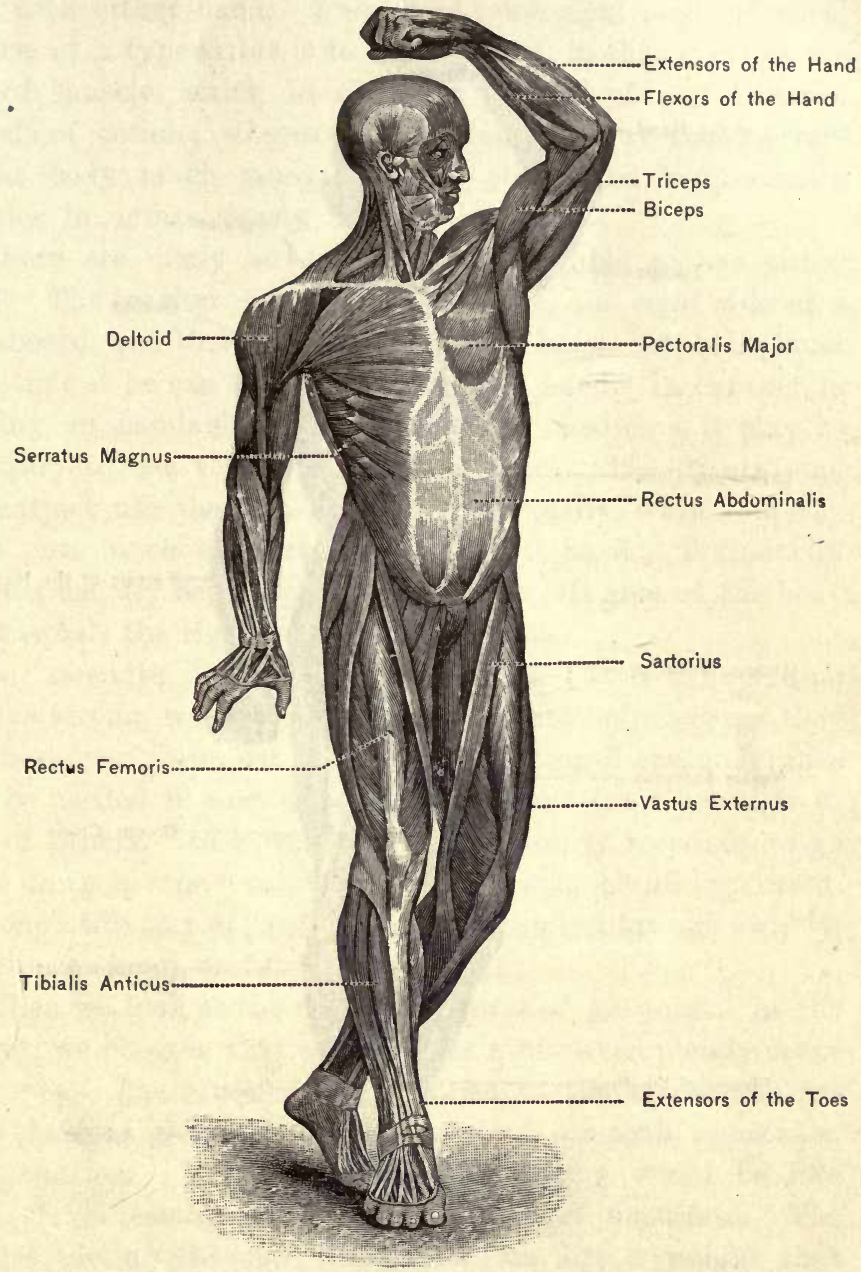

Ventral View of the Superficial Muscles.

To face page 18. 


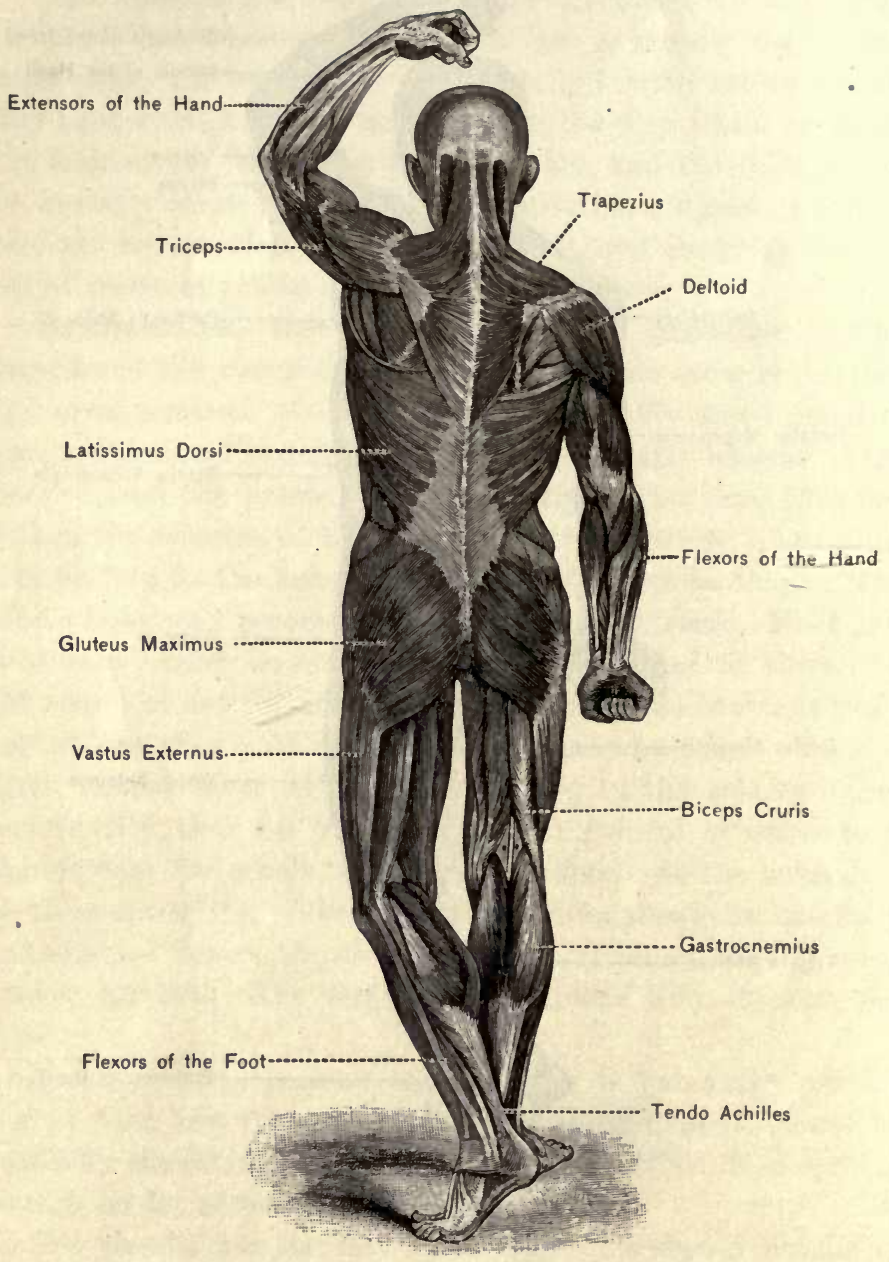

Dorsal View of the Superficial Muscles. 
well with either hand. From a physiological point of view, the use of a typewriter is to be preferred, in that it obeys the law of muscle action in allowing periods of rest between periods of action; whereas, in holding a pen we use one side of the body much more than the other, and keep certain muscles in action nearly all the time.

There are many advantages in being able to use either hand. The teacher may need to stand on the right side of a blackboard, and therefore to use his left hand. A ball-player loses time if he can throw with only one hand. In carving, in shaving, in bandaging, in administering medicine, it may be necessary to use the left hand skillfully. The pianist and the harpist use the two hands about equally, while the violinist puts much more skill into his left hand. Trainers of athletes usually begin by developing the left side of the body till it equals the right in size and strength.

Our strength depends on our muscles. It is a fine thing to have strong, well-developed muscles, not only because they give beauty of form, but because extra strength and endurance may be needed in case of accident, to save one's own life or that of others. In a case of fire, the ability to climb, to go up or down a rope "hand over hand," may be all-important. Any one's life may depend on his ability to run far and swiftly, to swim, to jump, to lift a heavy weight.

When we look at the skinned carcass of an animal in the market, we observe that the muscles almost completely cover the bones. The muscles are attached to the bones, and act upon them as levers, giving our motions strength, quickness, and precision. Without bones our motions would be like those of an earthworm or slug, slow and uncertain. The muscles, acting through the bones, can lift a weight that would crush the muscles if laid directly upon them; while a bone, able to support a heavy weight without being crushed, 
has no power in itself. The muscles have active strength, the bones have passive strength.

Since there is such an intimate relation between the muscles and the bones, let us turn to the study of the latter.

Relation of the Muscles and the Bones. - Suspend the skeleton from the ceiling in the most open space in the room. Let the pupils study it, book in hand; not to learn the names of all the bones, but to get a general idea of the forms and relations of the different parts. It is well to have the skeleton constantly at hand, to show the location of the various organs as they are taken up one at a time during the term. If possible, supply the class with separate bones from another skeleton, and let the pupils place each separate bone alongside the corresponding one in the complete skeleton.

Pass to the skeleton, and locate the biceps muscle. After examining Fig. 2, show the points of its origin and insertion. Feel the biceps of your arm. Note that its thickest part is opposite the most slender part of the bone. But at the enlarged end of the bone the muscle has narrowed to a slender tendon, which passes over the joint to be attached to the next bone, thus giving more slenderness, flexibility, and freedom of motion to the joint.

Take the bones of the arm that are articulated (if there is not an artificial hinge at the elbow, one can readily be made of wire); put a strong rubber band in place of the biceps muscle; fasten this to the head of the humerus by cords, and by the lower end to the radius and ulna, where the rough places, an inch or so from the elbow joint, show the insertion of the tendons. Have the rubber stretched so that when not held it will flex the forearm. This will serve to show the action of the biceps; though we must be careful to bear in mind that the muscle does not pull the arm up because it has been stretched, as is the case with the rubber. In the case 
of the muscle, we know that the live muscle has the power of shortening when stimulated, and in this respect is totally unlike the rubber.

The action of the bones of the forearm as a lever (we say lever, not levers, because the two bones of the forearm move as one in flexing and extending the forearm upon the arm) may perhaps be better understood by the following considerations: If the arm consisted merely of the biceps, suspended from the shoulder, it is evident that its only action would be a straight pull. Suppose the biceps, thus hanging alone from the shoulder, had a hook at its lower end, it could, when it shortened, lift a weight just as far as it shortened, and no farther. It could not swing the weight outward, or push it upward. But from the way in which the biceps is attached to the bones of the forearm, when the muscle shortens an inch it may move the hand a foot. Of course the hand moves much faster, and we have a great gain in speed by reason of this lever arrangement. Of course we must see that we cannot lift so heavy a weight at this faster rate as we could at the elbow. For instance, suppose one were to carry a heavy basket with a bail handle by slipping the arm through the bail up to the elbow. Now, it is evident that the biceps is supporting the weight. If it is as heavy as can be held here, we know that we could not hold the same weight in the hand with the elbow bent at a right angle.

Levers. - The essentials of a lever are, a point about which the lever turns, which in the body is some joint; the Power as it is called, which in the body is a muscle pulling, and a weight to be moved (not always to be lifted). The point about which a lever turns is called its Fulcrum. 'The place where the power is applied is called the Power (where the muscle is attached), and the part to be moved is the Weight. In flexing the forearm, the weight is the hand, or the 
hand and what is in it; the fulcrum is the elbow joint; and the power is the point where the tendon of the biceps is attached to the radius. This kind of a lever is what the books call a lever of the third class. The triceps, on the back of the arm, pulls on the projection of the ulna back of the elbow. The elbow is here, also, the fulcrum, and the hand (or the object to be pushed by the hand) is the weight. This kind of lever, where the fulcrum is between the power and the weight, is called a lever of the first class. In raising the weight of the body, by standing on tiptoe, we use a lever of the second class. Here the ball of the foot is the fulcrum. The weight is the weight of the whole body, resting on the ankle joint, while the power is the calf muscle. We may find many examples of levers in the body if we look for them.

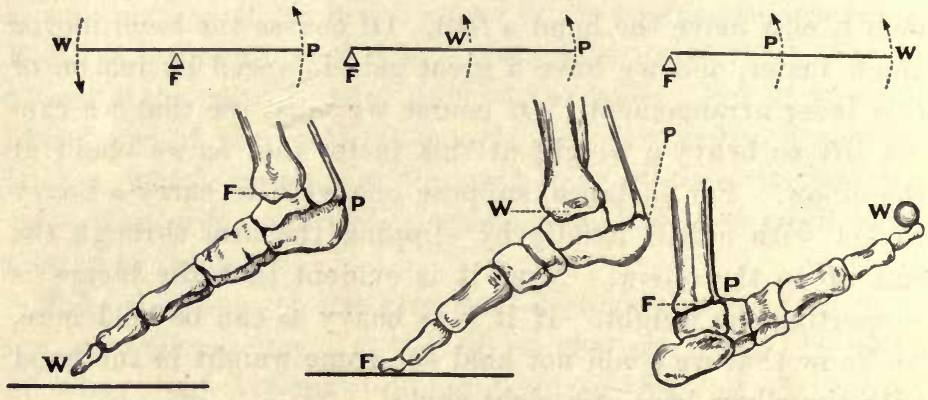

$\begin{array}{ll}\text { (1.) TAPPING ON FLOOR } & \text { (2.) RISING ON TOE }\end{array}$

(3.) LIFTING WEIGHT

Fig. 6. Three kinds of Levers as shown by the Foot.

P, Power. W, Weight. F, Fulcrum.

The different classes of levers may be further illustrated. by different motions of the foot. In tapping the toes on the floor while the heel is lifted, or in pressing down the ball of the foot while running the treadle of a sewing-machine, we have an example of a first-class lever. In raising the weight 
of the body on tiptoes, or as the foot is used in taking each step, the foot is used as a lever of the second class. When one lifts a weight with the toes, the foot is used as a lever of the third class. These three classes of levers are illustrated in the accompanying figures.

In the earthworm we have an example of what sort of motions we could execute without bones. And even the earthworm has spines that help it in crawling. But we see how slow and relatively feeble are its movements. Compare these with the rapidity, force, and precision of our muscular actions.

In the experiment with the frog's muscle we saw what we have mentioned as the action of a muscle by itself. We need levers through which the muscles can act.

Study of One of the Long Bones. - For this take, preferably, a femur or a humerus. Let us suppose we have a femur.

1. Observe its shape, - cylindrical, somewhat curved, en-

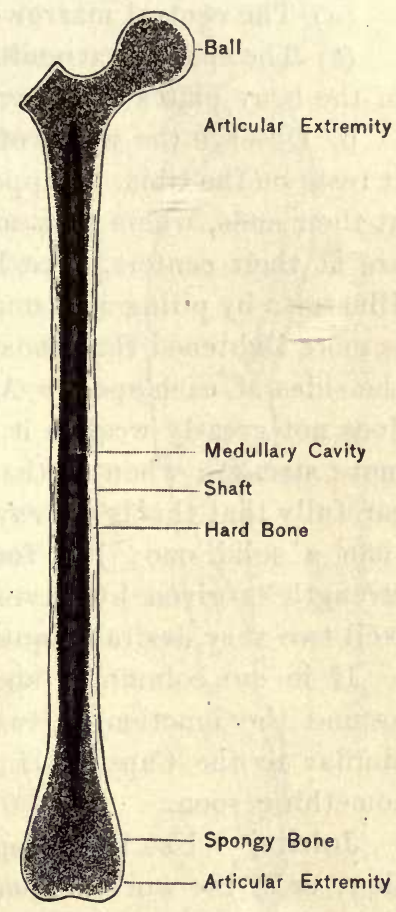

Fig. 7. Longitudinal Section of Femur. larged at the ends.

2. The ends have smooth places, where they fitted other bones.

3. Along the sides, especially near the ends, are ridges and projections, where the muscles were attached. 
4. There are small holes in the bone, where blood tubes passed in and out.

5. Saw a femur in two, lengthwise, and make a drawing showing:-

(a) The central marrow cavity.

(b) The spongy extremities, noting especially the directions of the bony plates and fibers.

6. Observe the width of the lower end of the femur, where it rests on the tibia. Suppose these two bones were as narrow at their ends, where they meet to form the knee joint, as they are at their centers, what kind of a joint would they make? Illustrate by piling up a number of spools on end; the column is more lightened than it is weakened by the hollowing out of the sides of each spool. And the central hollow of the spool does not greatly weaken it. A given weight of material has more strength when in the form of a hollow cylinder. Note carefully that this is not saying that a hollow pillar is stronger than a solid one; but for the same weight of bone more strength is given by having it hollow. The bones combine well two very desirable qualities, lightness and strength.

If in our column of spools we place a wide rubber band around the junction of two spools, we have something very similar to the Capsular Ligament, of which we shall learn something soon.

Joints. - Use beef joints or sheep shanks for demonstration; or, if the work is done by the individual members of the class, supply them with the legs of rabbits.

1. Cut into the knee joint. Observe the liquid Synovia that lubricates the joint. Rub a drop of it between the thumb and finger.

2. Note the white, glistening bands, the Ligaments, that hold the ends of the bones together. Carefully study their arrangement. The ligaments are composed of a form of connective tissue. 
3. Observe the thin layer of Cartilage over the ends of the bones. Feel it. Cut off a thin slice. Consider how its smoothness and elasticity give ease of motion, and diminish the shocks that the body receives.

4. Thoroughly clean one of the long bones, and study its form and parts. Its tough covering is the Periosteum.

Locomotion. - By continuing such observations as we made when we began to study our motions, we can analyze and understand many of the common movements which we habitually make.

Although we are not ordinarily conscious of the fact, when we are standing still we are using many muscles. The accompanying figure illustrates how some of the muscles act in keeping the body upright. Our weight, or, we would better say, the force of gravity, is continually trying to pull us down to the ground. The joints are all freely movable, and hence as soon as the muscles cease to act properly, in balancing against each other, we lose our equilibrium, and fall if we do not quickly regain it.

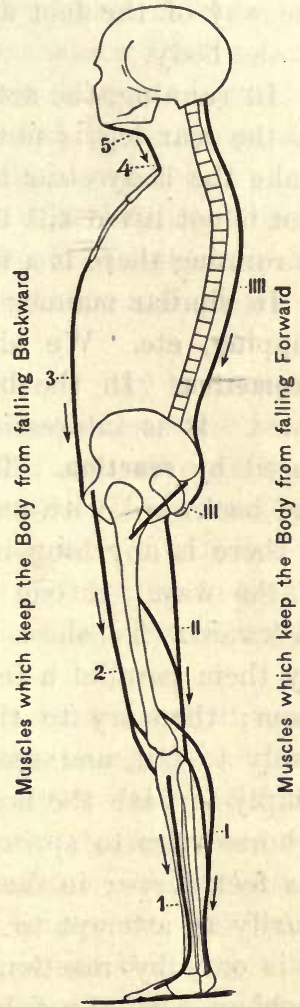

Fig. 8. Action of the Muscles in Standing.

In walking, we lean forward, and if we take no further action we fall. But we keep one foot on the ground, pushing the body forward, while the other leg is flexed, and carried forward to save us from the fall. We 
catch the body on this foot, and repeat the action. To show how we are really repeatedly falling and catching ourselves, recall how likely one is to fall if some obstacle is placed in the way of the foot as it moves forward to catch the weight of the body.

In running, the action is more vigorous. The propulsion by the rear leg is now greater. It gives such a push as to make the body clear the ground, whereas in walking the rear foot is not lifted till the front foot touches the ground. But in running there is a time when both feet are off the ground.

In similar manner we may explain the action in jumping, hopping, etc. We should distinguish between motion and locomotion. In the latter we move the body from place to place. It is interesting to note that our locomotion is produced by reaction. The direct result of pushing downward and backward with one leg is simply to extend the leg. Or, if there is anything in the way, to push that something out of the way. If one walks on a barrel the barrel is rolled backward. To show this better, take two broomsticks, and lay them parallel a few feet apart; lay a long board across them; then try to run along the board. Of course one is likely to fall, and sees that the direct effect of the effort is simply to push the board out from under one's self. When a horse tries to spring forward in the mud, he only plunges his feet deeper in the mud. Our effort in progression is primarily an attempt to push the earth out from under us, and it is only by reaction that we go forward. It is the same problem with the fish swimming forward by striking backward and sideways against the water, and in the case of the bird, beating downward and backward in the air. In each case progression comes only through the mechanical principle of reaction.

The muscles, then, make use of the bones as levers We 
do not ordinarily carry handspikes and crowbars with us; we get them when we need them : but we carry these levers with us all the time. Hence the desirability of having them as light as is consistent with the requisite degree of strength. The body follows the same law of mechanics that we use outside of the body. A pillar or hollow tube has a greater strength than the same amount of material in the form of a solid cylinder. The long bones of the limbs are hollow; and near their ends, where we have found that they need to be enlarged, we find a spongy appearance, where lightness and strength are secured by the interlacing fibers and plates of bony material.

As we have seen, the part that the bones play is of a passive nature; they support the other organs, protect some parts, and serve as levers on which the muscles act. We may not call the bones dead organs, for they receive blood and grow. But the active muscles use them as a man uses a crowbar, as a mere tool. It will therefore be more interesting to return to the muscles, and try to learn the causes and conditions of their activity.

Have you ever seen two persons, each using the right hand, try to sew, one holding the cloth, the other using the needle? Would they get along well? Suppose one were to hold the needle, and the other were to try to thread it, each using one hand? In dissecting, suppose one holds a delicate object with forceps while the other uses the scissors? Or that A holds the violin and does the fingering, while $\mathrm{B}$ manages the bow? Or in so comparatively simple a matter as the use of the knife and fork in eating, how if one holds the piece of meat with the fork while the other tries to cut it?

Why is it that the right hands of two persons cannot work so well together as the right and left hands of one person? What connection is there between the two, that one 
knows just what the other is doing and when it does it? Why can two individuals never, with any amount of practice, work so in unity as the parts of the individual?

Let us seek the answers to these questions in the following lessons.

Reading. - How to Get Strong and How to Stay So, Blaikie; Sound Bodies for Our Boys and Girls, Blaikie; Physiology of Bodily Exercise, Lagrange. 


\section{CHAPTER III.}

\section{THE GENERAL FUNCTIONS OF THE NERVOUS SYSTEM. - SENSATION AND MOTION.}

We have seen that the muscles have the power of shortening; that in shortening they act on the bones as levers to produce our varied motions. But what makes the muscles shorten?

Some motions we will to make. We will to sit, to stand, to walk, to run, to stretch out the hand, to take pencil and paper, to write. Such motions, originating in a brain activity, are called Voluntary. Other motions are Involuntary. The will does not control the heart-beat. Most persons cannot keep from winking when a quick motion is made toward the face, even if they know they will not be hit. But all of these motions, whether voluntary or involuntary, are dependent upon the nervous system. In the experiment with the frog's muscle we made it shorten by pinching the sciatic nerve. Let us look at the nervous system of the rabbit, which is larger than the frog's, and essentially like our own.

The Brain and Spinal Cord of the Rabbit. - It will be found helpful to have at hand a well-mounted skeleton of a cat or rabbit. Note carefully (a) the cavity of the cranium, (b) the cavity in the spinal column, and $(c)$ the sides of each neural ring where the bone is to be cut by the bone forceps, as indicated in Fig. 10.

It is best to remove the skin completely before beginning the work, as the fur is likely to be troublesome.

Cut away the muscles from the back of the neck and along 


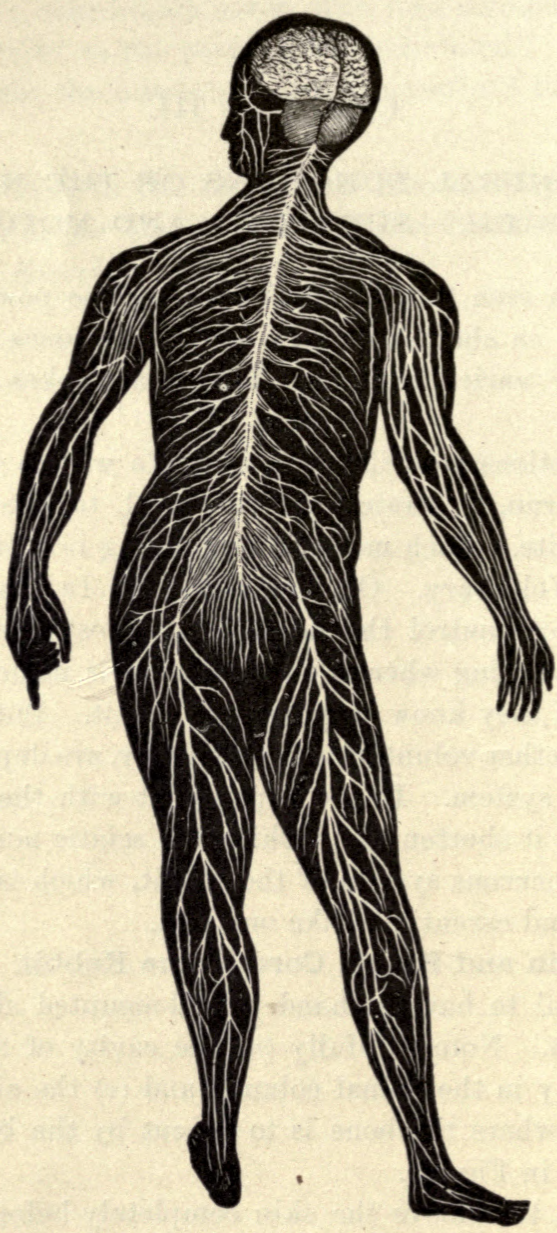

Fig. 9. Diagram Showing Arrangement of Nervous System. 
the sides of the backbone. This can be done rapidly by making long, deep cuts with the cartilage knife along the sides of the backbone, in the planes indicated in the accompanying figure.

Between the skull and the first vertebra is a space covered by a thin membrane, through which the Spinal Cord may be seen. Carefully cut through this membrane, and insert the point of one blade of a pair of bone forceps at one

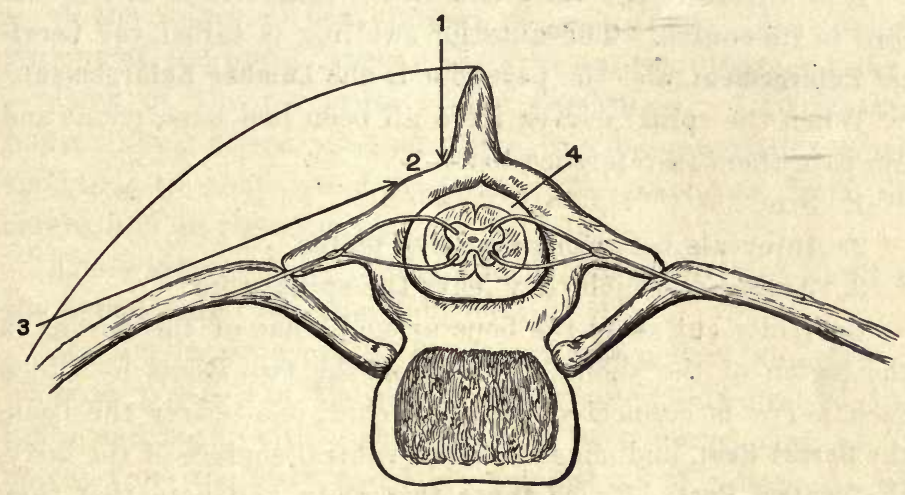

Fig. 10. Diagram for Dissecting Spinal Cord.

1. Cut along 1.....2 with cartilage knife.

2. Cut along 3 ....2 with cartilage knife.

3. Cut along 4 with bone forceps.

side of the spinal cord. Cut through this side of the arch of the vertebra; repeat this on the other side, and so on, through the whole length of the spinal column, removing the dorsal parts of the vertebræ, held together in one strip by the connective tissue. The bony cavity in which the spinal cord lies is the Neural Cavity.

The work may be more easily done if the rabbit is supported on the edge of a brick, or, better, on the edge of a 
short piece of "two by four" scantling nailed to a base-board eight inches wide and a foot and a half long.

Now look for the Spinal Nerves, which leave the spinal cord in pairs, right and left, between the successive vertebræ. It will probably be necessary to cut away considerably more bone to expose the nerves. The whole of this work requires the utmost care and patience, and involves a good deal of hard muscular exertion.

Note carefully the variations in the diameter of the spinal cord in its course. The anterior swelling is called the Cervical Enlargement, and the posterior is the Lumbar Enlargement.

When the spinal nerves have all been laid bare, count and compare them in reference to:-

1. Size.

2. Intervals between successive pairs.

3. Angles at which they leave the spinal cord.

Carefully cut away the bone around some of the nerves in the region of the shoulder, and find the two Roots by which each nerve is connected with the cord, one nearer the back, the Dorsal Root, and one nearer the ventral surface of the body, the Ventral Root. Trace these two roots, and note that they unite and form a spinal nerve.

On the dorsal root, just before it joins the ventral, is a small swelling, the Ganglion of the dorsal root.

In the region of the shoulder earefully trace several of the nerves as they unite to form the Brachial Plexus, from which nerves supply the fore limb.

In the region of the hips trace several of the spinal nerves to their union in the large Sciatic Nerve, which runs down the thigh.

Turn now to the head, and cut through the bone between the eyes. Cautiously working backward, the whole of the brain may be unroofed. Great care must be exercised, for 
here we have one of the softest of the tissues of the body lying very closely beneath one of the hardest. It is possible to do this work with a strong knife, but the bone forceps save a vast amount of extra work. The bone must be broken away bit by bit.

Compare the color of the brain with that of the spinal cord. The tough membrane covering the brain is the Dura Mater.

The fore part of the brain is the Cerebrum. Note the groove separating it into the right and left Hemispheres. Observe the ridges, or Convolutions, of its surface. The prolongations of the brain between the eyes are the 0lfactory Lobes.

Back of the cerebrum is the Cerebellum. Look at the human skull to see whether there is a bony partition corresponding to that which separates the cerebrum from the cerebellum in the rabbit.

The widening part of the spinal cord within the skull is the Spinal Bulb (Medulla Oblongata).

Make a drawing of the brain and spinal cord, showing as many as possible of the points above noted. If desired, the brain and cord, with a short part of each nerve, may be removed from the body, and laid on a cushion of cotton in weak alcohol.

Structure of Nerves and Nerve Fibers. - When we trace the sciatic nerve outward, we find that it is continually subdividing. This dividing goes on until the branches are too small to be seen by the naked eye. Microscopic examination shows that a nerve is made up of a great number of fibers bound together in a common sheath of connective tissues very much the same as in a muscle. When the nerve divides it is found that, ordinarily, there is no true branching, or forking, but that certain of the fibers have simply separated from the rest, as in the separation of the fibers in floss silk. 
An individual nerve fiber is too small to be seen by the naked eye, being only about one two-thousandth of an inch in diameter. 'There are two kinds, white and gray. A White Nerve Fiber consists of the following parts :-

1. A central strand, or core, of semi-transparent, grayishlooking material, called the Axis-cylinder. This is the essential part of the nerve fiber, and does the work of the nerve, which is to convey Nerve Currents, or Nerve Impulses.

2. Around the axis-cylinder is a layer of white, oily material, known as the Medullary Sheath.

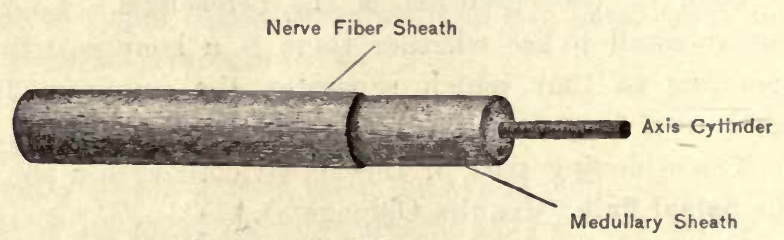

Fig. 11. Structure of a Nerve Fiber. (Diagram.)

3. Outside this is a thin, transparent sheath of connective tissue, essentially like the muscle-fiber sheath, the Nerve-Fiber Sheath.

The sole function of the nerve fiber is to convey nerve im. pulses.

Gray Nerve Fibers have no medullary sheath, but consist simply of the axis-cylinder and the nerve-fiber sheath. They are found principally in the sympathetic nerves.

Cross-section of the Spinal Cord. - If a thin slice of the spinal cord be made as shown in Fig. 12, it will be seen that the central portion is of a darker color than the outer part.

The central part is known as the Gray Matter, in distinction from the rest, which is called the White Matter. The white 
matter of the nervous system is made up of nerve fibers whose structure and use we have just considered. But the gray matter has a different structure and a different function. Instead of being made up mainly of fibers, it is composed of cells, rounded masses, some of the forms of which are represented in Fig. 13. Some of the branches of these cells are continued, and become the axis-cylinders of nerves, and it is asserted

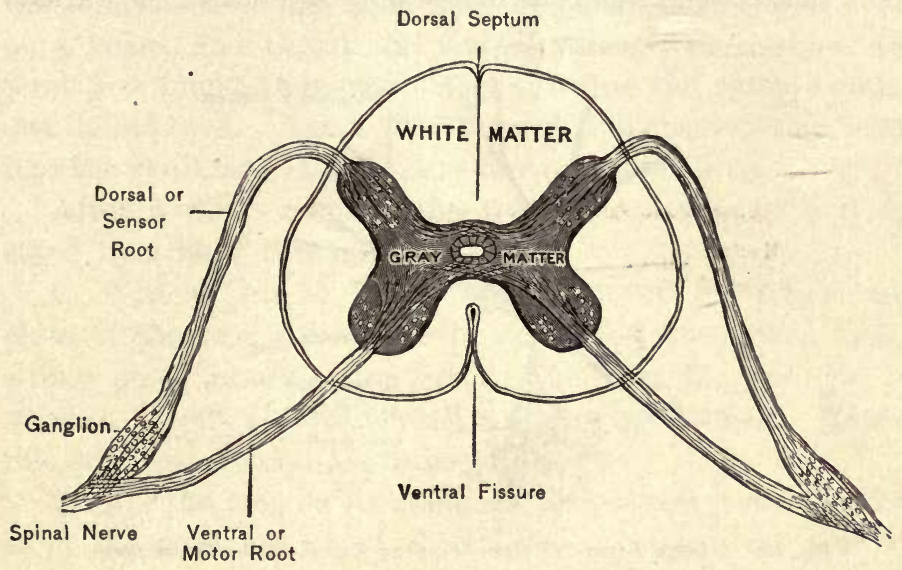

Fig. 12. Cross-section of Spinal Cord.

that every nerve fiber begins as a branch of some nerve-cell. One of the best places to see these nerve-cells is in the gray matter of the spinal cord, near where the ventral root of the spinal nerve arises. This part of the gray matter is called the Ventral Horn of the gray matter. If this portion be examined under a moderately high power of the microscope, there may be seen a number of cells with radiating branches. 
Masses of nerve-cells make up Nerve Centers, or Ganglia, such as we saw on the dorsad roots of the spinal nerves. These also would show under the microscope that their chief constituent is a collection of nerve-cells which give off one or more branches.

The gray matter of the spinal cord is considered a collection of ganglia. We see that the outer layer of the brain is grayish in color. Within is white matter, consisting of nerve fibers that connect the cells of the gray layer with the various

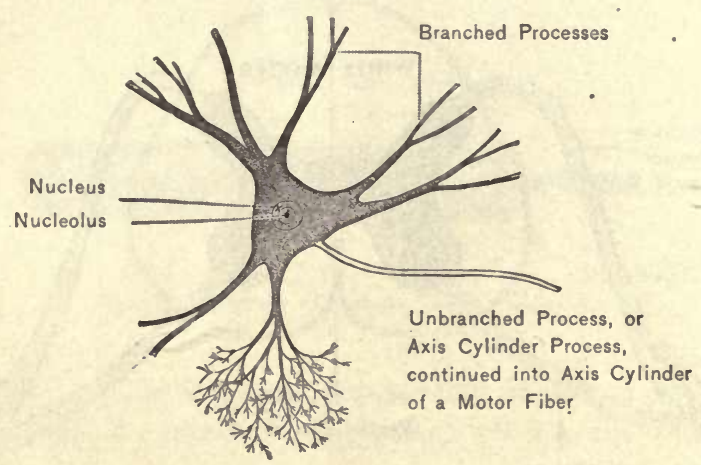

Fig. 13. A Large Nerve-Cell from the Gray Matter of the Spinal Cord.

parts of the body through the base of the brain, the spinal cord, and spinal nerves.

Reflex Action of the Spinal Cord of the Frog. - Before we experiment on the frog, let us recall some facts that we all know. After a fowl's head is cut off it "flops" around for some time, and it may even jump clear from the ground. If one takes hold of its feet to pick it up, it may begin to struggle as if it were trying to get away.

Now, we know that the bird cannot feel anything after its head is cut off, not even if a pin were thrust into its body, 
nor if its feet were held in fire. So with the frog. After its head is cut off, or, what amounts to the same thing, its brain destroyed, it cannot feel anything, and, of course, cannot have any pain. So, if in any of the experiments with the frog after its head is cut off it seems to be suffering or attempting to get away, remember, it cannot feel.

Kill the frog as directed on page 13. As soon as it is motionless, take it out of the jar. By bending its head, find the joint between the head and the backbone. Lay the frog on a board, and thrust the scalpel through the body at the joint just found, thus completely severing the spinal column and spinal cord. Run a wire through the opening thus made into the skull, and stir about to destroy the brain.

After a while suspend the frog from a ring of a retort stand by a hook through the jaw.

1. With a pair of forceps pinch one of the frog's toes. Now, if the frog were alive it would feel the pinch, and a strong pinch would cause pain. Now that the brain is destroyed, it feels nothing. Still it draws up the foot. Repeat the experiment with the other foot.

2. Lay the frog on its abdomen, and dissect away the skin from the back of one of the thighs. Separate the muscles along the noticeable groove in the posterior dorsal part of the thigh, using for this work the handle of the scalpel. There should be found the white, thread-like Sciatic Nerve. Carefully loosen this from the surrounding tissues, and pass one blade of a pair of sharp scissors under it. Steady the hand, so as not to give any motion to the suspended frog from the act of cutting, and, while closely watching the foot, cut the nerve. The muscles of the leg shorten, and jerk the foot.

3. Again pinch the toes of the limb whose sciatic nerve is cut. It does not move. Pinch the toes of the other foot; the leg draws up as before. 
We can now understand, that when anything touches our toes it starts what is called a Nerve Current or Nerve Impulse, which runs up the nerve to the spinal cord, and through this to the brain. If the brain is entire and awake, we have what we call a sensation of touch.

The feeling seems to be in the foot; but there is no feeling in the foot. Without the brain there is no sensation. If the nerve has been cut no sensation is produced, because the nerve current fails to reach the brain. It cannot pass the break in the nerve any more than an electric current can pass if the wire be cut.

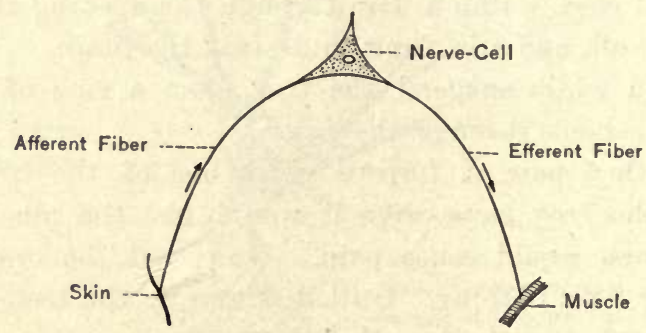

Fig. 14. Scheme of Reflex Arc. (After Landois \& Stirling.)

But our frog has lost his brain; he neither feels nor wills to do anything. How is it that he draws up his leg when his toes are pinched?

4. Run a wire down the cavity of the spinal column. This destroys the spinal cord. During this operation it will be seen that the muscles of nearly the whole body are set into violent action. This is because nerve impulses are produced by this irritation of the motor nerve-cells of the cord which pass out to the muscles, causing them to shorten.

5. Pinch the toes again. The foot is no longer drawn up.

The muscles and nerves are still able to act, as is seen on pinching the end of the nerve still connected with the foot. 
Such actions as the struggling of the chicken whose head has been cut off, and of the brainless frog in drawing up the foot when the toes are pinched, are examples of Reflex Action of the Spinal Cord.

When the toes are pinched, the nerve current, or nerve impulse, runs up the nerve to the spinal cord. The gray matter in the central part of the spinal cord receives the message; and sends back a nerve impulse to the muscles to make them shorten, and pull the foot away.

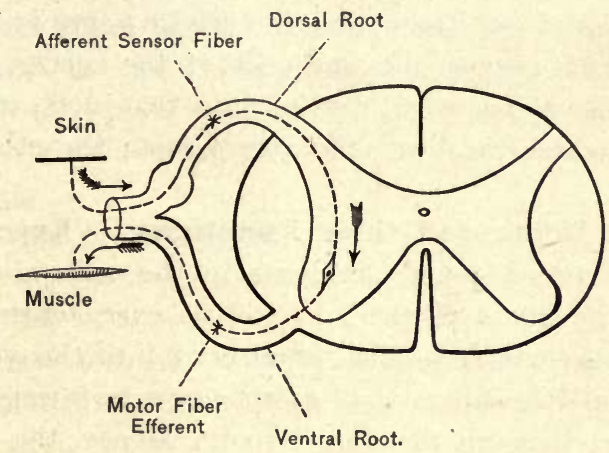

Fig. 15. Diagram of Reflex Action of the Splnal Cord. (After Landois \& Stirling.)

The sciatic nerve is composed of many fibers. If this nerve is traced outward, it is found to be continually subdividing, and sending small branches to the muscles, and finally in the muscles one fine nerve fiber goes to each muscle fiber. Many fibers go on past the muscles to the skin. We can feel in any part of the skin, and we can tell just where we are touched. These fibers from the skin, then, carry nerve impulses inward, as those going to the muscles carry impulses outward.

In skinning an animal we see many bands of white or transparent tissues connecting the skin and the sheaths of 
the underlying muscles. This is connective tissue, as is the sheath of the muscle. We also see blood tubes running out to the skin. And if we were to look very closely, we might see fine nerve fibers passing from the skin to join the nerve trunk in the legs and body. To skin an animal alive, then, would mean terrible torture; because all these nerve fibers would be cut or torn across, and every injury to the skin, or to the nerve fibers running inward from it, sends to the brain nerve impulses that, in the normal, live animal, arouse sensations of pain.

We have already seen that the sciatic nerve is made up of several spinal nerves, and that each of the nerves, just before entering the spinal cord, divides into two roots, one entering the cord nearer the back, the dorsal root; the other the ventral root.

Nerve Roots and their Functions. - Experiments on the lower animals, and accidents in the case of man, show that all the fibers of the nerves that carry currents to the muscles pass out from the spinal cord into the ventral root, and that all the fibers that carry currents inward enter the spinal cord through the dorsal root. Hence, the dorsal root is often called the Sensory root, and the ventral the Motor root. Since the dorsal root always carries currents inward, it is also called the Afferent root, while the ventral root, always carrying currents outward, is called the Efferent root.

Experiments have shown that if, in an unirijured animal, a nerve, or more properly a Nerve Trunk - as the sciatic nerve, - be stimulated, for instance, by a suitable electric shock, two effects are produced: first, rotion in the parts whose muscles are supplied by the nerve; second, sensation, which is referred to the parts of the skin supplied by the branches of the nerve.

If, instead of simply stimulating the nerve, the nerve is 
severed, the same two effects will be produced. We have seen that if we now stimulate the end of the nerve still connected with the limb, we get action of the muscles in that limb. If we stimulate the end of the nerve connected with the body a sensation will be produced, and this sensation will be referred to the parts from which the nerve fibers arise, probably in the skin of the limb.

If we now turn to the roots of the nerve, and try similar experiments, we get the following results: Stimulating the dorsal root causes sensation referred to some outer surface, and no other effect is noticed. Cutting the dorsal root also causes sensation. Stimulating the end of this root still connected with the spinal cord causes sensation; but stimulating the end of the root connected with the nerve gives no appreciable result.

Stimulating or cutting the ventral root causes motion in the parts whose muscles are supplied by fibers from this root. After severing this root, if the end connected with the spinal cord be stimulated, no effect is noticed; but stimulating the end still connected with the nerve is followed by shortening of the muscles supplied.

Severing all the spinal nerves destroys all power of sensation and voluntary motion in all parts of the body except the head. After severing all the dorsal roots, no sensation would be produced by stimulating any part of the body, and after severing all the ventral roots no act of the will can cause any of the muscles of the body to act. Severing all the nerves, or severing all the roots, cuts off all communication of the brain and the body, and so far as motion and sensation in the body generally are concerned, has the same effect as severing the spinal cord below the head.

The parts essential to reflex action of the spinal cord are:- 
1. A sensitive surface (the skin, for instance).

2. Afferent nerve fibers.

3. A nerve cell, or cells, in the center of the spinal cord.

4. Efferent nerve fibers (usually many).

5. Working organ, as muscle or gland.

In the experiment performed the steps in order were:-

1. Stimulation of the nerve endings in the skin of the toe.

2. Passage of a nerve impulse up the afferent fibers to the spinal cord.

3. Reception of the impulse by a cell, or cells, of the gray matter in the cord.

4. Sending back nerve impulses

5. Along efferent fibers of several nerves, to

6. Muscles which shorten and move the foot.

It is important that we understand the nature of reflex action, for very many of the processes of the body are regulated by it. Not only the more manifest motions, such as winking when anything comes quickly toward the eye, dodging, jumping when suddenly touched by anything hot or when pricked by a pin, when startled by a sudden loud noise, making a quick effort to regain one's balance when stumbling or slipping; when asleep, brushing away a fly or drawing up the foot when it is exposed to the cold - not only are all these reflex actions, but it is through reflex action that there are brought about the necessary continual adjustments of the essential processes of life,-circulation, respiration, digestion.

Cramp is a spasmodic shortening of the muscles, attended with pain.

Tetanus (a word also used for lock-jaw) is a spasmodic and continuous shortening of the muscles, causing rigidity of the parts they supply. It is due to the disordered and excessive stimulation of the muscles through the nerves. 
Functions of the Spinal Cord. - The spinal cord has two main functions:-

1. Its Conducting Power, by means of the white fibers which make up the outer part of the cord. These fibers may be regarded as connecting the gray matter of the brain with all parts of the body.

2. The gray matter is the Center of the Reflex Actions of the cord.

In looking at the cross-section of the spinal cord, we see that the dorsal and ventral grooves separate it into right and left halves, connected by a comparatively narrow isthmus. In this narrow part are fibers connecting the two halves. The brain also consists of right and left hemispheres connected by white fibers. Now, the gray matter of the outside of the cerebral hemispheres is considered the chief seat of both sensation and voluntary motion.

Further, most of the fibers which connect this gray matter of the cerebral convolutions, cross over to the other half of the spinal cord on their way to that part of the body with which they connect. Thus, when we wish to move the right hand the action starts with certain changes taking place in certain cells of the gray matter of the left cerebral hemisphere. Certain fibers extend from these cells down to the base of the brain, cross in the spinal bulb to the other half of the spinal cord, and run down in the right half of the spinal cord to the nerve leading to the muscles to be controlled.

Similarly, the sensory nerves cross on their way to the brain, only they are believed to cross soon after they enter the spinal cord. For instance, the fingers of the right hand touch an object. The nerve impulse thus started runs up the afferent fibers of the nerve of the arm. As it reaches the spinal cord it passes along the fibers of the dorsal root, and enters the dorsal part of the cord. It then crosses, prob- 
ably very soon, to the left half of the cord, and thence up on that side to the left half of the brain, rousing certain cells of the cerebral convolutions to an activity that we call a sensation of touch.

Voluntary Interference with Reflex Actions. - We have seen that the jerking of the hand away from a hot object is due to reflex action of the spinal cord. But it is possible, by act of will, to prevent, to a certain extent, the execution of reflex acts. One might, by a powerful effort of the will, keep the hand on an object that is hot enough to burn the skin. But we frequently see examples that are not so extreme as this. One may command the foot to remain quiet when it is tickled; he may keep himself from scratching a part of the skin which is irritated; but as soon as the person is asleep the same stimulations would be followed by the reflex actions such as we have considered.

In these cases of interference it is understood that the brain sends a nerve impulse down to the centers of the reflex action, and stops or diminishes their operation. This retarding influence of a group of cells is called Inhibition. It is not always due to voluntary interference, but may be due to reflex interference, as we may see later.

The Nature of a Nervous Impulse. - Of the nature of a nerve impulse we know but little. It is convenient to compare the nervous system, with its conducting fibers and central ganglia, to a telegraph system. And electricity is the most convenient stimulus for exciting nerve impulses. Yet a nerve impulse is very different from an electric current. A nerve fiber is a poor conductor of electricity. An electric current may travel along a copper wire at the rate of between 100,000 and 200,000 miles a second, while a nerve impulse in a motor nerve travels only 170 feet in a second.

When a motor fiber is stimulated in the middle of its 
course we observe only one effect, - the shortening of the muscle at its lower end. But there is every reason to believe that the nerve current, or impulse, runs along the nerve in both directions from its starting-point. But while the action of the muscle at the peripheral extremity manifests the existence of the current, there is nothing at the central extremity to give such evidence.

Similarly, when a sensor nerve fiber is stimulated, we have a sensation in the brain due to the current brought by the afferent fiber, and which we refer to the outer ends of the nerve fiber. Probably a nerve impulse passed from the point of stimulation to the outer end of the fiber; but as there is nothing at the outer end of the nerve fiber to interpret it, we get no evidence of such impulse except by refined physiological tests.

Harmony in Muscle Action. - Let us try to see what takes place in our bodies when we throw a stone at a mark. Many processes are involved, such as seeing, holding the stone, etc. Let us confine our attention chiefly to the act of throwing. A number of muscles are used. Each one of these must shorten in the right way and at the right time or the throw will not be accurate. Each muscle shortens under the influence of a nerve impulse started by the brain and brought by a motor nerve. If some muscle shortens an instant too soon, or a little too strongly, the stone goes to one side.

When we listen to a fine performance on the piano, there are certain things that even those of us who are not musical understand. We know that the right keys must be struck; that each must be struck at the right time, with the right degree of force, and held for the proper length of time, or else we have a harsh jangle of sound instead of sweet harmony.

What the player is to the instrument, that the brain is to the body. 
The cells of the brain are sending messages to the muscles. There must be a distinct series of nerve impulses for the control of each muscle. The impulses must be in the proper sequence and of the right degree of strength to make each muscle shorten just at the right time, with the right force, for the right length of time, or we shall have muscular confusion instead of orderly and accurate movements.

In playing a game of temnis the brain is an organist with five hundred keys before him. And before he has finished the tume, he has had occasion to use nearly every one of them, especially if the player is not only dextrous but ambidextrous.

The precision and rapidity of these movements are most wonderful. Imagine an organist with so many keys, or a telegraph operator sending out so many messages over so many wires in so brief a space of time, and getting them all right. Yet we have learned all this. The new-born babe has little more than the power and the tendency to move. Most of his acts are not orderly. He gradually learns to hold up his head, to sit, to grasp objects, to walk, to throw, and execute all the co-ordinated movements that we now discover to be primarily under the management of the two Master Tissues (as Foster calls them), Nervous Tissue and Muscular Tissue.

Dependence of Nerves and Muscles. - It may have happened to you that after sitting long in one position you attempted to stand, but found that you could not do so. One leg failed to act at the bidding of your will. Pressing on the foot caused little, if any, sensation.

Or, perhaps, you may have awakened in the night and found one arm numb and unable to move.

When the foot is "asleep" we get little sensation from it; we hardly know whether it is touching the floor or not. Pressing on it with the other foot causes no pain. 
We try to stand when the foot is asleep, but we are unable to do so. The brain starts the nerve currents, and they run along the nerve as far as the compressed part; here they stop. They cannot reach the muscles of the leg below. Hence the muscles do not shorten, and we do not rise, no matter how strongly we will to do so.

Why is it that the nerves and muscles thus sometimes lose their ability to perform their natural activities?

This has been explained by saying that the nervè has lost, for the time, its power of conducting nerve currents, owing to external pressure. But is it not possible that some of the effect of pressure on the limb is indirect rather than direct? That something beside the nerve has been compressed, and that the apparent inability of the nerve to convey the nerve impulse is, in part, a secondary, and not a primary, effect? What process in the limb has been interfered with by the pressure due to the position in which one has been sitting or lying? What is the temperature of the benumbed limb?

On what are the nerves and muscles so dependent for the maintenance of their activity?

Reading. - Power through Repose, Call; The Technique of Rest, Brackett; Muscles and Nerves, Rosenthal. 


\section{CHAPTER IV. \\ CIRCULATION OF THE BLOOD.}

The Blood and its Work. - If the supply of blood to a muscle be shut off by tying or otherwise compressing the arteries leading to it, the muscle soon loses its power to act. If the blood supply is not too long checked, its readmission will restore the muscle to normal condition. If the blood is wholly shut off, say from one of the lower limbs, there would soon be loss, not only of muscular power, but of sensibility as well.

We know that if any animal is bled freely, it becomes weak, then unconscious, and soon dies, if the escape of blood is not stopped.

We observe the natural difference in color of different parts of our bodies; for instance, the lips and cheeks. We often note varying color, as in blushing and pallor.

We wish to understand these differences and changes; also to know what to do in case of fainting or bleeding from wounds. Our comfort, health, and often life itself, may depend on our knowledge upon this subject. We may prolong and make more useful our own lives and those of others by knowing, in a practical way, something about the causes, prevention, and remedies of the colds, congestions, and inflammations to which we are subject.

Nearly every part of the body bleeds when cut. There is no bleeding when we trim the nails or cut the hair, and the outer skin has no blood in it. But the inner skin, and almost every tissue within it, if pierced, even by the finest needle, 
yields blood. We see a little blood oozing from the surface of a fresh steak or roast.

What kind of a substance is the blood? Is it uniformly distributed through the tissues, like water soaked up into a cloth, or is it in distinct cavities? Why is it so essential to life? How does it do its work?

Let us seek answers to these questions.

\section{EXTERNAL INDICATIONS OF THE CIRCULATION OF BLOOD IN THE HUMAN BODY.}

The Heart-Beat and the Pulse. - 1. The heart-beat, felt at the left of the breast-bone.

2. The pulse, felt at the wrist and at various parts of the body. Perhaps the most convenient place to study it is at the temple. Lay the forefinger lightly along the cheek just in front of the ear. Count the pulsations for a minute.

Let one or two pupils who are quick at figures step to the blackboard and put down the number of pulsations of each pupil as they are called, and divide by the number thus reporting, to get the average.

1. Let all in the class count the pulse while sitting. Probably it will be best to discard the first trial, as there are likely to be several failures from one cause or another. Then, too, there is usually a slight excitement at the beginning of a wholly new experiment. Get the average of the class.

2. Find the pulse while sitting; rise quickly, and immediately begin to count the pulse. Compare with the pulse as taken while sitting.

3. Again find the pulse after sitting quietly for some time; rise quickly, begin counting immediately, and note the number at the end of a half-minute, but count on continuously to 
the end of the minute. Compare the number of pulsations in the first half-minute with that in the second half.

4. Stand and take active exercise for a few minutes, as if with dumbbells. Immediately count the pulse.

5. At home, count the pulse while lying on a lounge, after remaining in that position for half an hour.

6. Compare the pulse before and after meals.

7. With the thumb and finger lightly clasp the windpipe, well back. The pulse in the Carotid arteries will be felt.

Let all the pupils in the class stand. Let one arm hang freely by the side. Hold the other arm straight up as far as the clothing will readily permit. Observe:-

1. The difference in the color of the two hands.

2. The difference in fulness, both in the feeling of fulness and in the prominence of the veins.

3. The difference in temperature; place the backs of the hands against the cheeks.

The position largely determines the amount of blood in the hand, and the amount of blood determines the temperature, the size, and the color.

With the forefinger stroke one of the veins on the hand or wrist toward the tips of the fingers. The vein swells out. The blood meets resistance in the valves of the vein. Their location may be determined by their bulging out during the experiment.

Stroke a vein toward the body, and the blood is pushed along without resistance.

Let the left hand hang by the side. Note the large vein along the thumb side of the wrist. Place the tip of the second finger on this vein just above the base of the thumb. Now, while pressing firmly with the tip of the second finger, let the forefinger, with moderate pressure, stroke the vein up the wrist. It may be seen that the blood is pushed on freely, 
but comes back only part way. It stops where it reaches the valves, filling the vein full to this point, but leaving it collapsed beyond, as shown by the groove. Remove the second finger, and the vein immediately fills from the side nearer the tip of the fingers.

These experiments show that the blood in the veins moves freely toward the body, but cannot flow outward to the extremities.

With the finger-tips press on the back of the hand. Note the temporary white spot caused by the pressure.

Observe the color of the finger-nails in the closed (but not clinched) hand. Now straighten the fingers as far back as possible, and again note the color of the nails. Press the nail on its surface, ends, sides, and note the changes in color.

A Drop of Frog's Blood. - Kill a frog as directed on page 13. If blood enough is not obtained from the wound already made, open the body cavity and cut across the heart.

Place a small drop of blood on each of several slides and quickly cover with coverslips.

First examine with a low power, one inch, or half-inch objective. Then with a one-fifth inch objective. There will be seen :-

1. The Colored Corpuseles. They are yellowish pink in color, elliptical in shape. Some of them, seen edgewise, will be seen to be flattened, with a bulging center. This bulge is caused by the Nuclens.

2. The Colorless Corpuscles, few in number compared with the colored corpuscles, much smaller, ordinarily circular in outline, because they are usually spherical. They are almost always dotted in appearance. If watched closely for some time they may be seen to change their shape, like the Ameba. Watch these changes. Make drawings of the outline at intervals of ten seconds. 
The clear spaces between the corpuscles is filled with the liquid part of the blood, called the Plasma.

Our blood is very much like that of the frog, as we shall see a little later.

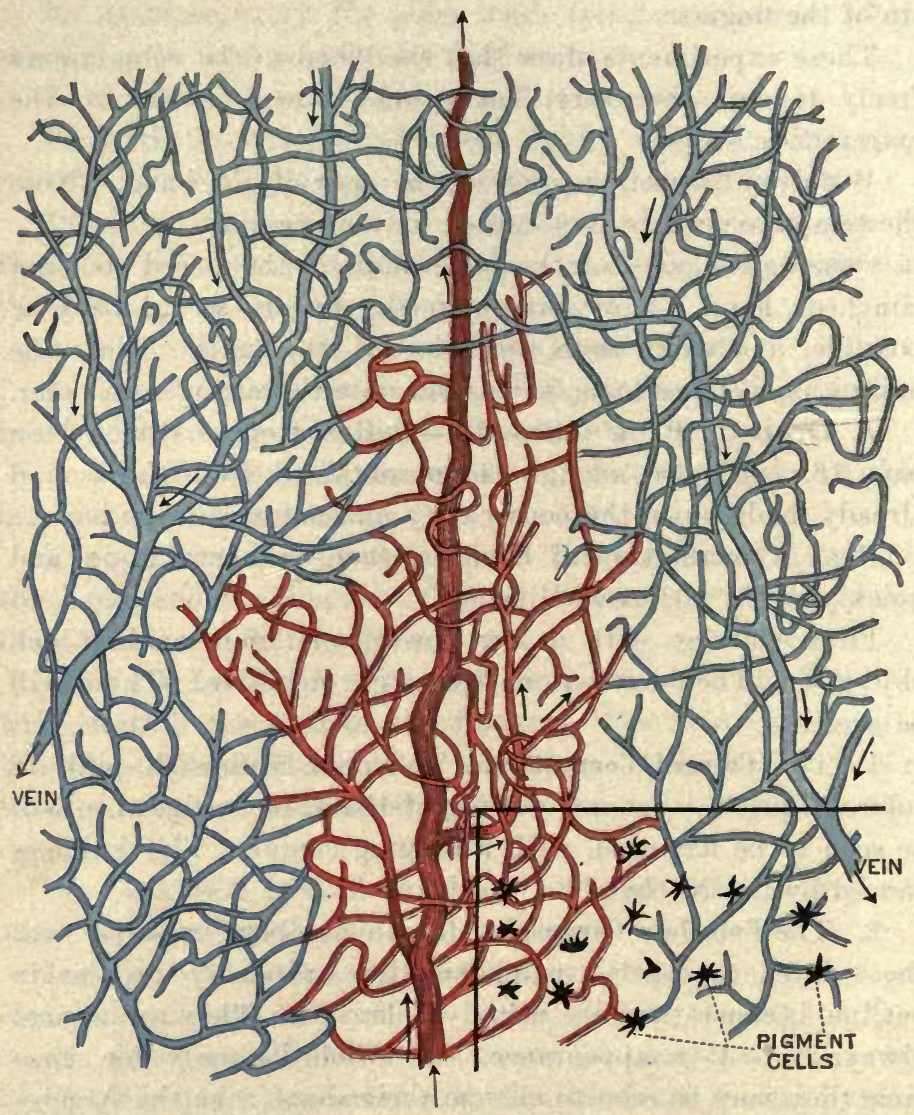

ARTERY

Fig. 16. Part of Frog's Web (low magnifying power). 
The Circulation of Blood in the Web of a Frog's Foot. - For this get a frog with a pale web. Take a piece of shingle six inches long and three inches wide. Cut a round hole, half an inch in diameter, near one end of it.

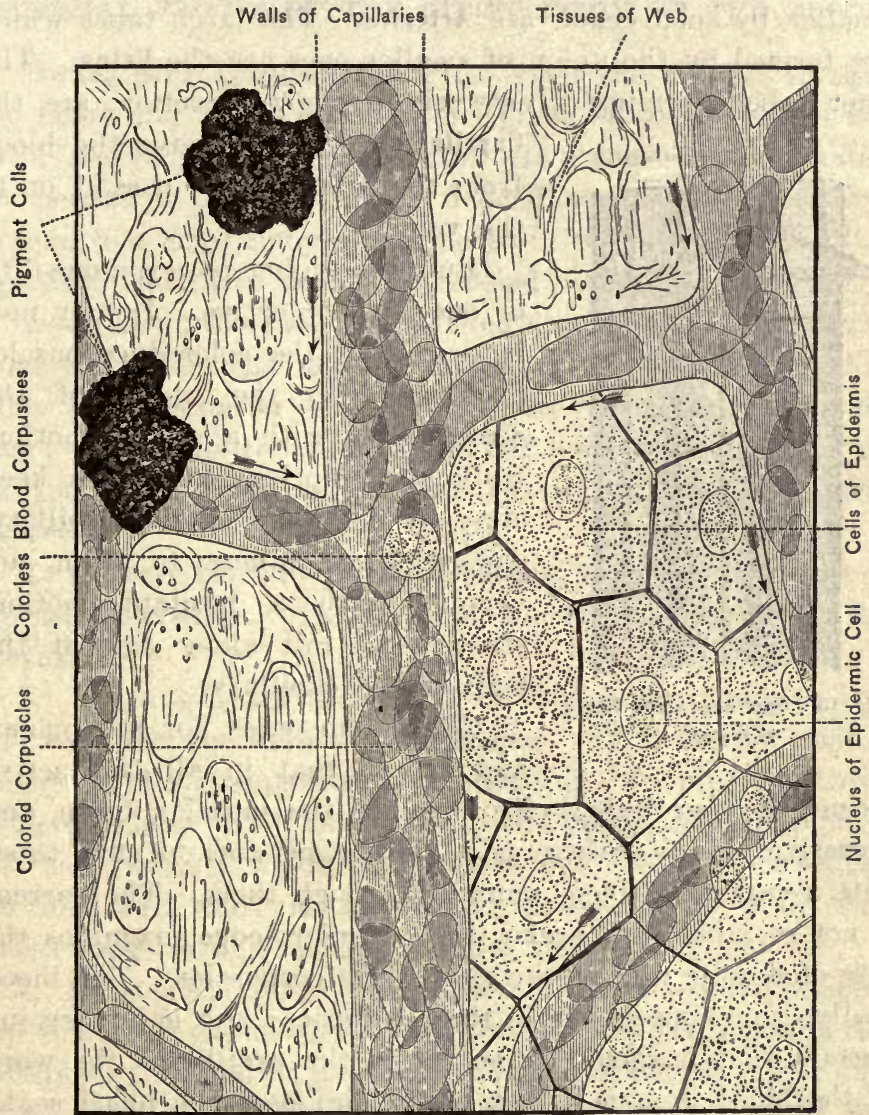

Fig. 17. Part of Frog's Web (highly magnified). 
Wrap the frog in a wet cloth, with one leg projecting, and tie it, thus wrapped, to the shingle. Tie threads around two of the toes, and stretch the web, but not too tightly, over the hole. Place the shingle firmly on the stage of a microscope. Examine first with a low power. The large tubes which grow smaller by subdivision are Arteries. The large tubes which are formed by the union of smaller ones are the Veins. The finer tubes, forming a network in every direction, are the Capillaries. They receive the blood

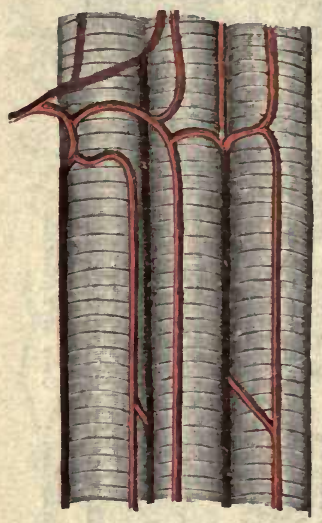

Fig. 18. Capillary Blood Tubes of Muscle. from the arteries, and pass it on to the veins.

Put on a higher power, a one-fifth or one-sixth objective. It may now be seen that the colored corpuscles float more in the center of the stream, and with a steady motion, while the colorless corpuscles keep close to the walls of the capillary, and seem to adhere to them, advancing with a hesitant motion, seeming to roll along against the wall of the capillary.

Close your eyes for a moment, and reflect that in all the active tissues of your body - for example, the muscles, brain, and digestive organs - there is a similar network of fine tubes with a current of blood running through them. The current is not so rapid as it seems, for the microscope magnifies the rate of flow as well as the size of the corpuscles. The blood really is moving slowly in the capillaries; and it is very important that it should be so, for in the capillaries the work of the blood is done. Part of the liquid of the blood soaks through the thin walls of the capillaries, and nourishes the 
surrounding tissues. All the other parts of the circulatory system exist for the purpose of sending a continuous, slow, and steady stream of blood through the capillaries.

INTERNAL PROOFS OF THE CIRCULATION OF THE BLOOD.

Heart and Lungs of a Pig. - Get the butcher to save the heart and lungs entire (the "pluck"), being careful not to cut the lungs or the heart case. If a considerable number are needed it is better not to depend on local butchers, but to send to a large slaughtering-house in the nearest city. The "plucks" should be thoroughly cooled before being shipped.

1. Hold up the mass by the wind pipe, with the heart away from you. The end now uppermost is the anterior end, that below is the posterior end ; the lung to your right is the right lung; the one to your left is the left lung; the surface nearest you is the dorsal surface, and that opposite is the ventral surface.

2. Observe the windpipe, or Trachea, with the stiff rings of gristle, or Cartilage. The thick part of the anterior end is the

\section{Larynx.}

3. Running along the dorsal surface of the windpipe is a soft red tube, the Gullet, or Esophagus. At about the middle of the windpipe separate the gullet and windpipe for three or four inches. Note that next to the gullet the windpipe is soft and yielding where the gaps of the C-shaped cartilages are filled with muscular and elastic tissue. Make a slit two inches long in this soft membrane.

4. Inflate the lungs as follows: Take a wooden faucet, slip the small end of the faucet into the slit just made in the windpipe, and hold or tie firmly, but do not cut off either gullet or windpipe. Inflate through the spout, then shut off the air; if the lungs have not been punctured they should now 
remain distended. In holding up the lungs, take hold of the windpipe above where it is tied, and not of the faucet. Note (a) the conical shape of the whole; compare this with the chest cavity, as shown in a skeleton; (b) how the lungs nearly surround the heart; $(c)$ the concave posterior surface of the lungs where they fitted the convex anterior surface of the diaphragin; $(d)$ the groove between the dorsal surfaces of the lungs in which the spinal column fitted; $(e)$ the smooth, undivided dorsal surface of the lungs, and their division ventrally into Lobes ; $(f)$ the relative lengths of the dorsal and ventral surfaces of the lungs. The anterior end of the lung is the Apex; the posterior end is the Base. Open the valve of the faucet. What makes the air go out? Again inflate. Does it require effort to do so? Why? Cut off the end of one lobe and again inflate. Does the air escape? Throw a piece of lung on water. Pinch a piece of lung, holding it near the ear. The smooth, moist, glistening membrane covering the lung is the Pleura.

5. Observe a large whitish or yellowish tube running in the groove between the dorsal surfaces of the two lungs. It is usually covered with fat, and if it has been cut off short its open end is easily seen near the windpipe. This is the main artery, the Aorta. Take hold of its free end, and separate it from its attachment to the other tissues, cutting close to it with the scissors, so far as where it arches over the root of the left lung. Now turn the free end forward.

6. Find where the gullet is cut off posteriorly; slit it open for an inch or two, and note its whitish lining, the Mucous Coat. The thick red coat is the Muscular Coat ; try to distinguish an inner layer of circularly arranged muscle fibers and an outer longitudinal layer. Beginning posteriorly, separate the gullet from the windpipe, cut off the windpipe about the middle, and entirely remove the gullet and larynx. 
7. Examine the windpipe; insert a finger, and stretch it; note its C-shaped cartilages. Its lining is a Mucous Membrane.

8. Lay the heart and lungs on their ventral surface, with the posterior end nearer you. Using the handle of the scalpel as a chisel, clear away any tissue covering the windpipe, and trace it to the lungs; its branches are the Bronchi. How many bronchi are there? Here are often found small, oval, brownish masses, the Lymphatic Glands, imbedded in connective tissues. Scrape these loose with the scalpel handle.

9. Lay the lungs on their dorsal surface, with the anterior ends toward you. Note how easily the heart may be moved about in its ease, the Pericardium. Slit the pericardium along its ventral side, and note the smoothness of its lining and of the surface of the heart. Observe the Pericardial Fluid.

10. Carefully compare the right and left sides of the heart. Running obliquely across the surface of the heart is a groove in which are blood tubes, often covered with fat. The part at the right of the groove is the Right Ventricle; at the left is the Left Ventricle.

11. At the base (anterior end) of the heart on each side are the right and left Auricles.

12. Tip up and toward you the apex of the heart. Compare its width and thickness; compare the ventral and dorsal surfaces as to length, convexity, etc. Compare its width and thickness; press the two ventricles, and compare them in firmness.

13. Turn the heart to the left, and examine the right auricle; find a large, flabby, red-lined tube entering its anterior surface, the Precaval Vein. Prick a small hole in it near the pericardium and insert the blowpipe; hold firmly around the opening and inflate the right auricle; the Postcaval Vein should now be discovered entering the auricle from the posterior 
region. Look for it outside, and on the dorsal side of the pericardium, where it runs anteriorly from the diaphragm.

14. Turn the heart to the right, and observe a large, lightcolored tube arising from the base of the right ventricle between the two auricles; this is the Pulmonary Artery. Again turn the heart to the left, and raise the right auricle; find the Aorta arising from the center of the base of the heart. Carefully separate the aorta from the pulmonary artery, and trace the aorta as it arches over the left bronchus, and runs down between the two lungs by the side of the gullet. Clear away any fat or other tissue adhering to it.

15. From the arch of the aorta arise the branches running to the head and forelimbs.

16. In the same way trace and clear the pulmonary artery.

17. When the fork of the pulmonary artery has been reached, lay the heart and lungs on their ventral surfaces, with the postericr end toward you; turn the windpipe back toward you, and with the scalpel handle trace the branches of the pulmonary artery into the lungs. Cut them off close to the lungs.

18. Keeping the parts in the same position, trace the Pulmonary Veins. These are to be found on the ventral side of the bronchi; their general outlines may be shown by inflating as follows: Cut off the first branch of the aorta as close as possible to the arch. Insert one blade of the scissors in this opening, pointing away from the heart, and make a slit two inches long. Insert a cork toward the heart. It should fit snugly, so that air may not escape. For a pig's heart a cork about three-fourths of an inch in diameter at its larger end is about right. Make a very small hole in the tip of the left auricle, insert the blowpipe, holding firmly around it, and inflate. This should distend the left auricle and the pulmonary veins. With the haudle of the scalpel scrape away 


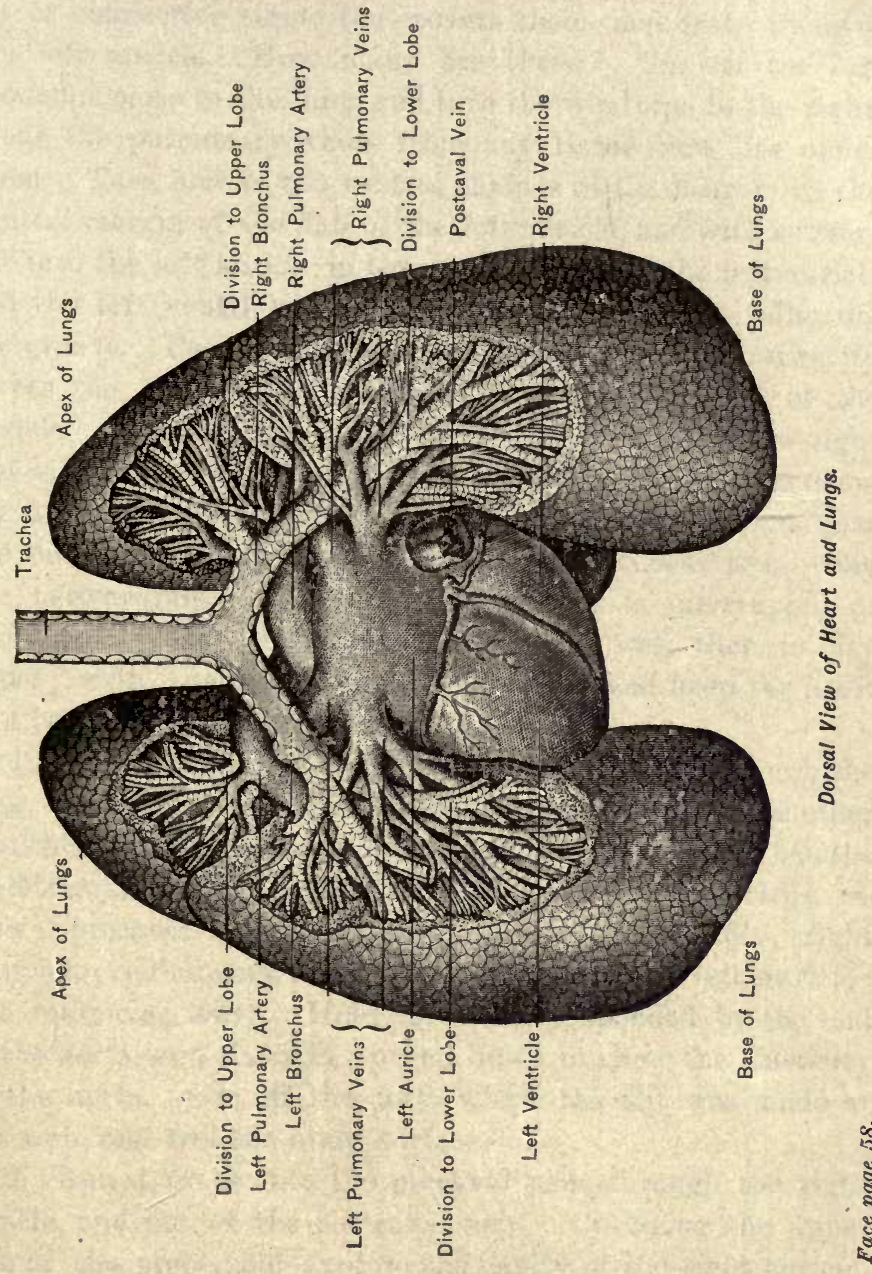



fat or connective tissue that covers them, and trace them to the left auricle. How many are there? Cut off the left bronchus close to the lung, and turn the windpipe to the right. Clear the pulmonary veins from any tissue that lies under them. Turn now to the ventral surface of the heart; lift the ventral margin of the flap of the left auricle, and with scissors cut into the left auricle in the groove between the left auricle and the left ventricle. Make a slit an inch long, following the groove. Pass a probe through the opening, then directly across the cavity of the auricle, to the dorsal wall of the auricle. Here are the entrances of the veins from the right and left lungs. Use the finger as a probe, enlarging the opening a little if necessary. Cut off the pulmonary veins near the lungs, cautiously avoiding other blood tubes; trim away the pericardium. If the preceding work is interrupted at about this point, wrap the heart first in wet, then in dry paper; write your name upon the wrapper, and keep the heart in a cool place for later study.

19. With the scissors slit down one bronchus through the lung, noting its branches. Follow the outside of another bronchus, tearing away the lung tissue with the scalpel handle.

Structure and Action of the Heart. - 1. Briefly review: precaval vein; postcaval vein; right auricle; right ventricle; pulmonary artery; pulmonary veins ; left auricle; left ventricle; aorta. Hold the heart suspended by the end of the aorta, and dance it up and down to show the elasticity. of the aorta. Cut off the aorta where the slit was made at the arch, and feel the inner surface.

2. Run a probe into the precaval vein, through the right auricle, and out of the postcaval vein. Cut along the upper side of the probe, and explore the cavity of the right auricle. Feel the inside of the auricle and veins. Observe that the cavity of the auricle extends farther into the heart than the 
notch between the auricle and ventricle. At the extreme left of the right auricle is the mouth of the Cardiac (coronary) Vein, which, running around between the left auricle and left ventricle, brings blood from the ventral wall of the heart. Near the mouth of this vein also empty the veins seen in the dorsal wall of the heart. Pick out any clots that may be found. Slit the anterior wall of the auricle, being careful not to cross the groove between the auricle and ventricle, and note the muscular columns within the appendage of the auricle.

3. Cut away the whole of the appendage of the right auricle. Remember that the pulmonary veins from the right lung run very close to the right auricle, and be careful not to cut into them. Pin down the flap of the left auricle, so that water may not enter the left half of the heart in the next experiment. Hold the heart in the left hand, with the ventral surface in the palm, and the tips of the fingers against the right ventricle. Hold the heart under a faucet, or pour from a pitcher, and let the water run first gently, then strongly, through the right auricle into the right ventricle. Watch the Tricuspid Valves as they float up and separate the auricle from the ventricle. Empty the heart and fill it again, and as soon as the valves rise, press with the fingers on the outside of the ventricle. What effect has this pressure? Let the nozzle of the faucet project down between the valves, and again turn on the water. Where does the water escape?

4. Empty the heart and examine the valves. They will be found lying close against the walls of the ventricle. Note the white cords, Chordæ Tendineæ, attached to the valves.

5. Push the finger past these valves to the very bottom of the ventricle; from the outside cut through the wall of the ventricle at this point, and cautiously cut upwards in both directions along the border of the ventricle. Raise the outer 
wall of the ventricle, and study the valves more thoroughly; with the scalpel handle raise them from the walls of the ventricle. How many flaps are there? How are they arranged? The conical elevations of the muscle to which the chordoe tendinece are attached are the Papillary Muscles. How are the valves held in place? How are they acted on, and how do they act?

6. Find the connection between the right ventricle and the pulmonary artery; pass a probe up into the pulmonary artery. Cut away enough of the wall of the ventricle to show the beginning of the artery. Cut off the pulmonary artery just before it forks to the two lungs; slip over the faucet the end of the artery connected with the heart, and turn on a little water. Watch closely the base of the artery; turn on more water, and look from below at the base of the artery, to see the filling of the pocket-like Semilunar Valves. Note their number, shape, and arrangement. What is the effect of the stream of water upon them, and what is their effect upon the stream of water?

7. Examine the left auricle, and find where the pulmonary veins enter it. Cut away the lobe of the left auricle; examine its inner surface, and find the openings of the pulmonary veins. Hold under a faucet, and prove the action of the Mitral Valve, between the left auricle and the left ventricle. Insert the nozzle of the faucet between the valves, and again turn on the water. Where does it escape? Cut off the aorta half an inch from its base, and repeat the last experiment with the water, meanwhile closely watching the semilunar valves of the aorta.

8. Above the pockets of the semilunar valves look for the openings of the Cardiac (coronary) Arteries, which supply the walls of the heart. Probe them. How many are there?

9. Pass the handle of the scalpel between the semilunar 
valves of the aorta into the left ventricle; it passes back of one flap of the mitral valve.

10. Cut open the left ventricle. Note the strong muscular columns, the strong papillary muscles; the mitral valve, though ending in two main flaps below, is continuous at the top. Compare the walls of the right with those of the left ventricle. Why this difference? Note the partition between the ventricles. Is there any direct communication between the right and left halves of the heart?

11. Slit open the aorta between two of the semilunar valves, and study the valves more closely. In the middle of the free border of each valve note the little thickened point, the Corpus Arantii. When the valves close, these three little points fill up a small three-comered opening that would otherwise be left between the valves. Again examine the cardiac arteries.

12. In another heart, carefully cut around the base of the pulmonary artery, tie its outer end tightly over the end of a glass tube or spool, and show the action of the semilunar valves, by blowing suddenly and foreibly into the tube. To keep the glass tube from slipping out, slip an inch of thick rubber tubing on the end of the glass tube, so that the rubber tube is even with the end of the glass tube. The valves work better when moist and flexible; therefore keep the preparation standing in a jar of water until it is to be used. Slit open the artery, and study the valves.

13. Longitudinal and cross sections of a frozen heart are instructive.

Demonstration of the Action of the Heart. - Get the heart and lungs entire. Dissect out the aorta as before. Clear the pulmonary artery, and cut off both branches close to the lungs. Carefully trim away the pericardium, and clean the precaval and postcaval veins. Turn the heart back, and find 
one of the larger pulmonary veins; cut a hole in it near the lung, and slip a glass tube into it toward the heart. This tube should have a groove, made by drawing it out in the flame. Another, and perhaps easier, way to keep the glass tube from slipping out, is to slip over the end of the tube a piece of rubber tube an inch or two long; but it should not project beyond the end of the glass tube. If this fits snugly, it will not slip on the glass tube, and the blood tubes will hold firmly when tied over the rubber. It is much better to tie the tube into the pulmonary vein before the vein is cut off; otherwise there will be difficulty on account of the shortness of the pulmonary vein. Tie the tube firmly in, and ligature the other pulmonary veins without stopping to trace them. Tie all connections with the heart now remaining, and cut beyond the ligatures. Get a retort stand and two large glass funnels, or have made a more convenient piece of apparatus (as shown in Fig. 19), consisting of a sheet-iron pan eighteen inches square and two inches deep, with a fixed bail handle twenty inches high, made of iron rod of the size of a retort-stand rod. Attach retort rings and clamps to the rod, as shown in the figure. This whole apparatus, with the heart attached according to the directions given, can easily be carried, and any overflow of liquids will be caught by the pan. Place the funnels in the rings. Lay the heart, now wholly severed from the lungs, on its ventral surface. Connect one funnel by rubber and glass tubing, with the left auricle by the tube already in the pulmonary vein; connect the other funnel with the right auricle, through the precaval vein; ligature the postcaval vein. Lay the heart in a basin, and pour water into the funnels; hold the heart with the two hands, and compress it, repeatedly adding water. In this way the clotted blood usually present in the right ventricle may be washed out. If this remain, it may interfere with later experiment. Connect the aorta 
with the funnel which leads to the right auricle by means of a glass tube which bends over the edge of the funnel, thus holding itself in place by the hook, or held above by a clamp or ring, and emptying into this funnel any liquid which escapes from the tube.

In like manner have a bent glass tube, from the pulmonary

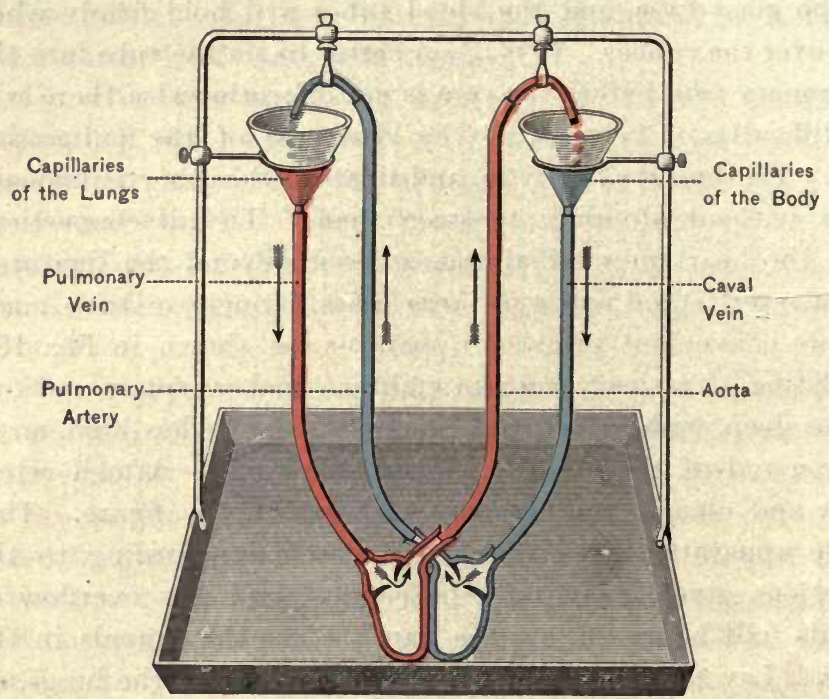

Fig. 19. Demonstration of the Action of the Heart (Heart Diagrammatic.)

artery, held by a clamp above the funnel leading to the left auricle.

If possible, let the heart soak in water over night before showing it to the class. This will loosen the clots, and make the valves more flexible.

Pour water into one of the funnels, and compress the heart to imitate its natural contraction; observe where the liquid 
next appears; add more water, and follow it around to its starting-point. A little ink may be poured into one of the funnels, and traced around, as the heart is worked, to its starting-point.

That there is no direct connection between the two halves of the heart may be shown by letting the liquid from each artery empty into the funnel connected with the auricle of the same side of the heart. Different-colored liquids may be used in the two funnels.

In order to illustrate more fully how the heart is composed of two pumps fastened together, and each pumping its own stream, but worked by the same power, try the following :-

Take the two funnels supported as in the preceding experiment; connect each funnel with the supply tube of a common bulb syringe; connect the delivery tubes with the bent tubes used with the heart.

1. Let each bent tube empty into the funnel from which it gets its supply. There are now two distinct circuits.

2. Now cross the delivery tubes so that each discharges into the funnel from which the other gets its supply. Now, on working the bulbs, we have a circuit like a figure 8 , really one circuit, but the two streams cross each other.

3. Again, place the two bulbs side by side, and work the two with one hand.

4. Wrap a cloth around the two bulbs, so that what is contained in the cloth cannot be seen. We have now a structure like the heart, but we know its structure. We know that it consists of two pumps wrapped together and working together; that is, by the same stroke, but with two wholly independent currents.

Reason for Injecting the Arteries. - The arteries and veins, unless distended with blood, are so nearly of the same 
color as the surrounding tissues that it is difficult to distin. guish them. Hence it is very desirable to fill them with some colored substance.

The following starch preparation, recommended by Wilder and Gage in their admirable work, "Anatomical Technology," has been found very satisfactory :-

STARCH INJECTION MSAS.

Dry starch ("Laundry" is good) . . . . . . 100 c. c. Water, or a $2 \frac{1}{2}$ per cent aqueous solution of chloral hydrate $100 \mathrm{c}$. c. Alcohol (95 per cent) . . . . . . . . . 25 c. c. Color mixture (as given below) . . . . . . 25 c. c.

"After thoroughly mixing the mass, it should be filtered through one or two thicknesses of moistened paper cambric. To prevent the starch from settling, the cloth should be tilted from side to side, or the mass may be stirred during the filtration. If the mass is not freshly prepared for every injection, the stock mass should be filtered occasionally, to remove hair or any other object that might clog the cannula.

"Since almost any animal injected may afford some organ worth preserving, it seems better to employ permanent colors in tingeing the mass. Among these which are available, the following, probably, are preferable : vermilion, red lead ultramarine, Berlin blue, chrome orange, yellow, or green.

\section{PREPARATION OF THE COLOR.}

Dry color . . . . . . . . . . . . 100 c. c.

Glycerin . . . . . . . . . . . . 100 c. c.

Alcohol (95 per cent) . . . . . . . . 100 c. c.

"To avoid lumps, which would clog the cannula or small blood tubes, the color should be thoroughly ground in a mortar. It should be stored in a well-stoppered bottle, and is prepared for use by simply shaking. If permanent preparations are 
not to be made, the mass may be stained by aniline of the desired color."

Excellent results have been obtained by the use of carmine in coloring the mass for injecting the arteries, and Berlin blue or Prussian blue for the veins.

Kill a cat or rabbit with ether or chloroform, by putting the animal into a tight box or jar with a sponge containing a teaspoonful of the anesthetic. When the animal is dead, open the thorax by eutting across the posterior ends of the breast-bone, and through the costal cartilages on each side, being careful not to cut the mammary artery which runs along the inside of the breast-bone on each side. The mammary artery should be ligated just under the anterior end of the breast-bone. Now cut away the breast-bone. The breastbone may be simply turned forward, and in this case it will not be necessary to ligate the arteries.

Find the aorta, and clear away any tissues that may obscure its base. Pass a ligature under the aorta here, but do not tie until the cannula is inserted. Cut a small slit in the apex of the left ventricle. Have in readiness several cannulas (or nozzles of a brass syringe) of different sizes, made by drawing out glass tubing. Each cannula should have a distinct neck, so that it may be tied in firmly. Insert the cannula through the ventricle into the

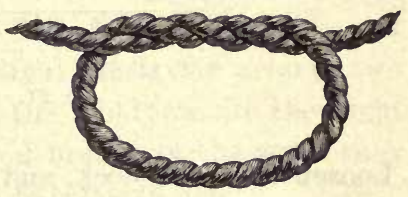

Fig. 20. Surgeon's Knot. base of the aorta. Now tie the cannula firmly by the surgeon's knot, made by crossing the two ends of the thread twice instead of once, as in the ordinary knot; draw firm with a slight sawing motion, but do not tie again. (See Fig. 20.)

For injecting, use a good brass syringe, if it can be had; a 
white metal syringe does fairly well, or the following method may be employed: Place a good-sized glass funnel in a large ring of a retort stand. Slip a piece of black rubber tubing over the tube of the funnel, and fasten it on firmly with cord or small wire. Put a pincheock on the lower end of the tube. Now pour the starch injection mass into the funnel.

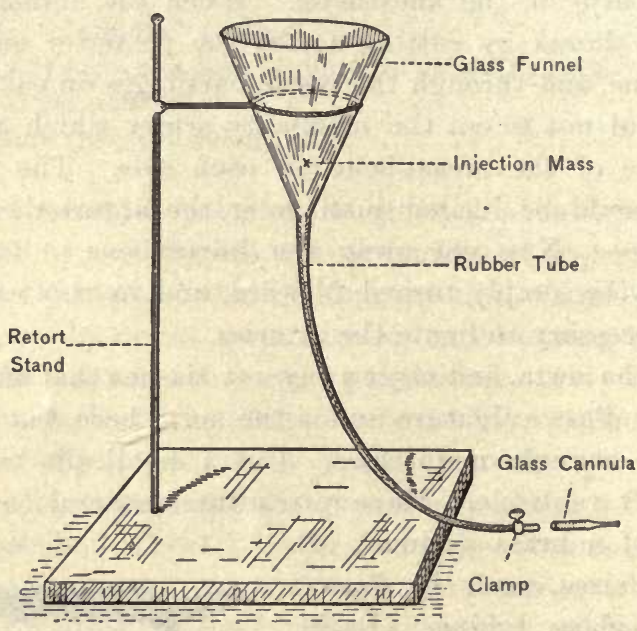

Fig. 21. Injecting Apparatus.

Loosen the pincheock, and let a little of the mass run out, to be sure that the tube is full. Now slip the end of the tube on the cannula, loosen the pincheock, and inject by "stripping" the rubber tube; or the process might well be described by the term "milking," as it is like that process. Hold the upper part of the tube with one hand, and with the other the mass may be driven into the aorta. One great advantage of this method over the use of a syringe, is that the operator 
constantly feels the degree of resistance; for the pressure is given directly by the fingers, instead of through a piston. Another advantage is that the retort stand holds itself; and if it becomes necessary to stop the pressure at any time, the pinchcock may be applied to the tube, and both hands are free for any needed adjustment, while everything is held in readiness to go on with the work after simply loosening the pinchcock. If a number of specimens are to be injected at one time, a separate cannula may be used for each one, and the lower end of the tube simply transferred from one to the other. If desired, the mass may easily be stirred, or emptied from the funnel. The cannula may be removed after ligaturing the aorta, or a piece of rubber tubing slipped over the cannula and tied. In any case, it is necessary to prevent reflow of the mass until it hardens.

The Distribution of the Arteries and Veins in the Cat or Rabbit. (Injected.) - 1. The main artery, the Aorta, is a thick-walled tube, springing forward from the center of the base of the heart. It soon arches over to the left, and runs along the middle of the dorsal wall of the chest cavity.

2. At the bend, or Arch, the aorta gives off two branches (three in man). The first of these soon subdivides, giving off a branch to the right forelimb, the Right Subclavian artery; two branches running along the side of the windpipe are the Right and Left Carotid arteries. The second branch of the aorta runs to the left forelimb, and is the Left Subclavian artery.

3. During its course through the thorax the aorta is called the Thoracic Aorta. Trace it to the point where it runs through the diaphragm. It then becomes the Abdominal Aorta. Turn the stomach and intestine over to the right, and observe the abdominal aorta running along the dorsal wall of the abdomen. Just posterior to the diaphragm, a branch is given off which subdivides, and gives branches to the stomach, liver, and spleen. 
Farther back a large branch is given off to the small intestines. Follow it as it branches through the Mesentery. This is the Anterior Mesenteric Artery. Find the branches of the aorta that lead to the kidneys, the Renal Arteries. Some other branches may be seen; and finally the aorta divides into two large branches, the Common Iliaes supplying the two hind-limbs.

4. Turn the stomach and intestines to the left, and observe the two veins running forward from the two hind-limbs. These are the two External Iliac Veins. By their union they form the Postcaval Vein (formerly called the Vena Cava Inferior).

5. Observe the veins from the kidneys, the Renal Veins.

6. Trace the postcaval vein to the liver. Observe the vein that gathers the blood from the intestine, the Mesenteric Vein. This vein is joined by a vein from the stomach, the Gastric Vein, one from the spleen, the Splenic, and one from the pancreas, the Pancreatic; together these form the Portal Vein, which empties into the liver. Unlike other veins, the portal vein subdivides, distributing the blood through the liver. The blood thus distributed through the liver is recollected, and by the Hepatic Veins joins the postcaval vein, close to the diaphragm, and almost wholly concealed by the liver.

7. The postcaval vein passes by the liver, through the diaphragm, and on to the right auricle.

8. On removing the skin of the neck, there should be found on each side the large Jugular Vein. Each of these is formed by the union of the Internal and External Jugular veins.

9. Just before each jugular vein enters the chest eavity it is joined by a vein coming from the corresponding fore-limb, the Right and Left Subclavian Veins. The union on each side forms the Innominate Vein. The two innominate veins uniting make the Precaval Vein, which enters the right auricle. In the rabbit there are two precaval veins. 


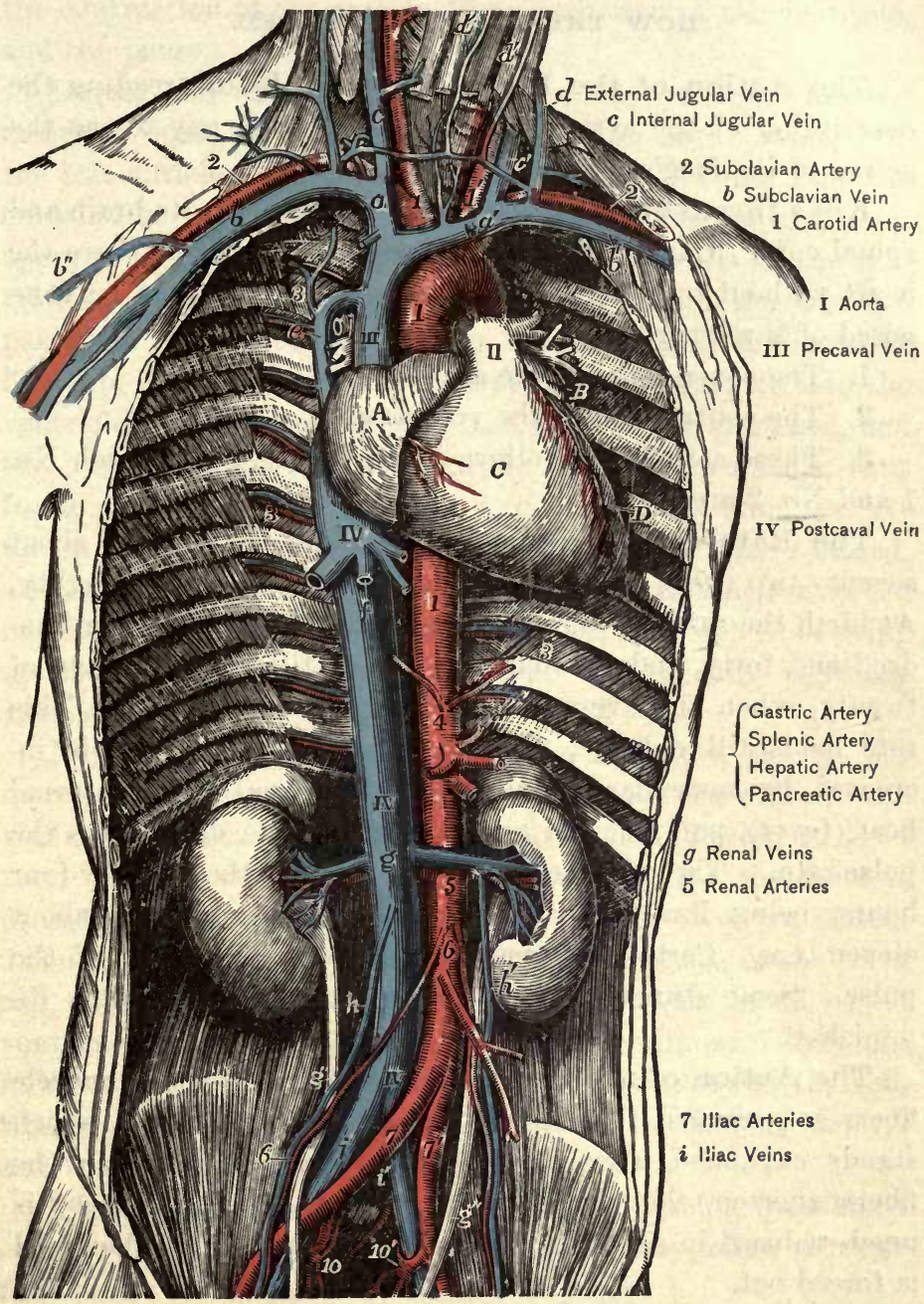

Fig. 22. Distribution of Arteries and Veins, 
HOW THE BLOOD IS PUMPED.

The Action of the Frog's Heart. - Before reading the description of the action of the heart, it will pay to see the action of the frog's heart.

Kill a frog as directed on page 13, and destroy its brain and spinal cord. Carefully open the body cavity, and lay bare the heart without injuring it. Its beats will be seen to be com. posed of three parts :-

1. The contraction of the auricle.

2. The contraction of the ventricle.

3. These actions are followed by a pause, after which No. 1 and No. 2 are repeated.

The Rate of the Heart-Beat. - The heart beats about seventy-two times a minute in men. In women, about eighty. At birth the rate is from one hundred and thirty to one hundred and forty, and gradually decreases till about the age of twenty, when the average of seventy-two is reached. This rate holds till old age, when it increases. The rate is increased by muscular activity, food, external heat, internal heat (fever), pain, mental excitement. Music accelerates the pulse-rate. The pulse-rate varies during the twenty-four hours, being lowest during the night, and highest about eleven A.M. Certain diseases increase the frequency of the pulse. Some drugs quicken the pulse-rate, and others diminish.it.

The Action of the Heart. - The heart consists of muscle fibers so arranged that they form a thick-walled bag, which stands expanded when the muscles relax. But when the fibers shorten, the whole heart contracts, and the cavity is much reduced in size, if not entirely obliterated, and the blood is forced out.

The complete action of the heart consists of three parts, 
the contraction of the auricle, the contraction of the ventricle, and the pause.

During the pause the blood is steadily pouring into the auricles; into the right auricle from the caval veins, into the left auricle from the pulmonary veins. At this time the curtain-like valves between the auricles and the ventricles are open, and their flaps hang loosely beside the walls of the ventricles. The blood, therefore, as it passes into the auricles, passes on into the ventricles. As the ventricle fills, the valves float up, as seen in the experiment of pouring water into the ventricle. When the ventricle is full, but not stretched, and the auricle partly full, the auricle suddenly contracts, thus forcing more blood into the ventricle, and distending it. At the same time the valves, which were already nearly closed, are tightly closed by the pressure of the blood which is forced up behind them. The flaps of the valves are kept from going up too far by the chordae tendinece and by the papillary muscles.

Next comes the contraction of the ventricle, slower, but more powerful, than that of the auricle. As the walls of the ventricle are drawn together, the blood is subjected to pressure. It cannot go back into the auricles, for the more it presses against the valves, the more tightly they are closed. The semilunar valves are closed by back pressure in the aorta and pulmonary artery. But the pressure of the blood in the ventricles is so much greater that the semilunar valves are forced open, and nearly all the blood is driven out of the ventricles; from the right ventricle into the pulmonary artery, and from the left ventricle into the aorta.

While the ventricles are contracting and forcing their blood out, the auricles are slowly filling by the steady inflow through the veins.

As soon as the ventricle has completed its contraction, it 
dilates, and most. of the blood that has accumulated in the auricle simply falls into the ventricle. The dilating ventricle exerts a slight suction, so the blood is in part drawn into the ventricle. During the remainder of the pause the blood accumulates in the auricle and ventricle till the auricle again contracts, and the cycle is repeated.

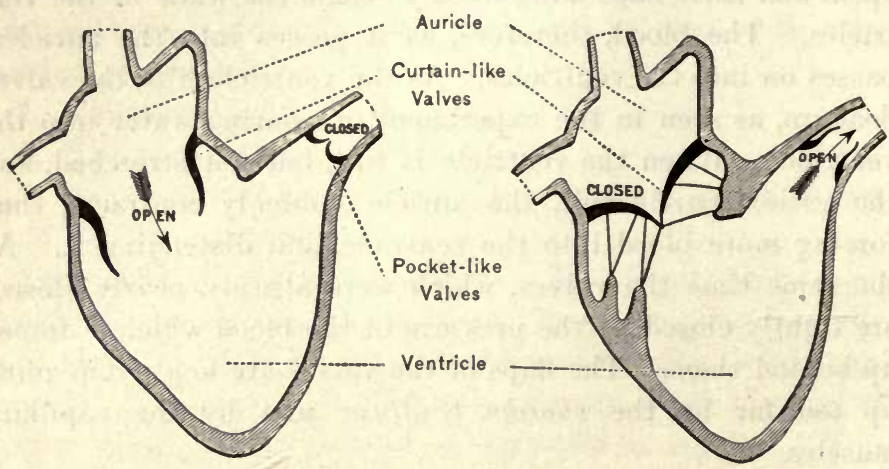

Fig. 23. Diagram of the Heart, Showing the Action of the Values.

The time taken by the different parts of the heart-beat, as near as can be measured, are about as follows :-

Since the heart beats seventy-one or seventy-two times each minute, each beat takes about eight-tenths of a second (.8); of these eight-tenths the auricle takes for its contraction oneeighth of the time; that is, one-tenth of a second (.1); the contraction of the ventricle occupies three-eighths of the time, or three-tenths of a second (.3), while the pause fills the rest of the time; that is, four-eighths, or one-half, of the whole time of the entire heart-beat, or four-tenths of a second (.4). Let us call the time required for the complete heart-beat the heart's day. Of course this day is very short, less than a second. If we suppose it to be twenty-four hours, we can 
more easily see how much of the time the heart is actually at work, and how much of the time the heart is resting:-

Auricle contracting (working) $\frac{1}{8}$ of the time $-3 \mathrm{~h}$, , resting $21 \mathrm{~h}$. Ventricle contracting (working) $\frac{3}{8}$ of the time $-9 \mathrm{~h}$., resting $15 \mathrm{~h}$.

No part of the heart, therefore, is working longer than a man would who only works nine hours a day. Some observers state that the resting period is even greater than these figures would show.

Since the contraction of the ventricles immediately follows that of the auricles, one-half of the time is occupied by the whole beat of the haart, and during half the time the whole heart is resting. This is different from our usual statements regarding the work of the heart. We hear it said that the heart never rests. Its work and rest follow each other at such short intervals that we do not appreciate the interval of rest that comes between the successive impulses that we feel. Suppose a policeman had the power of sleeping at will, and that he slept thirty minutes of each hour, and that in the remaining thirty minutes he made the rounds of a block. If we saw him passing regularly once an hour, every hour of the twenty-four, we might suppose that he did not sleep at all during the entire time.

The Beat of the Heart. - The apex of the heart is always in contact with the chest wall. Consequently, it never strikes it. At each beat it pushes hard against the chest wall. This push may be felt and seen, and is called the heart-beat.

The Sounds of the Heart. - There are two sounds of the heart: -

1. A short, sharp sound made by the closing of the semilunar valves.

2. Just preceding this sound a longer, duller sound may 
be heard, during the contraction of the ventricles. This is supposed to be due to the vibrations of the walls of the ventricles, and of the large valves.

Action of the Large Arteries. - The large arteries have in their walls a yellow elastic tissue. When the blood is forced into them they are stretched. As soon as the ventricle ceases to contract, and sends no more blood into the arteries, they "stretch back." We should not say contract, for it is simply an elastic reaction. As the artery reacts it presses on the blood, and hence the blood tries to escape in every possible way. It cannot go back, for it fills the pockets of the semilunar valves, and closes them with a click. A rapid wave is sent forward that gives the pulse, and a slower but still rapid strean flows along the arteries, through the pulmonary artery to the lungs, and through the aorta and its branches to all the other parts of the body.

The elastic reaction of the arteries thus makes steady the flow of blood which is intermittent as it leaves the heart.

Action of the Medium-Sized Arteries. - Each organ requires a supply of blood in proportion to

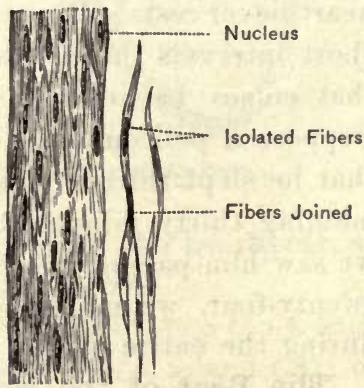

Fig. 24. Plain (Unstriated) Muscular Fibers from the Bladder. its activity. An actively working organ, like the brain, demands much more blood than bone practically inactive. Further, working tissues, such as the brain and muscles, need a great deal more blood while they are at work than when they are resting. An organ needing a constant large supply of blood might secure this by having a large artery. But how can the supply be regulated so that an organ may receive, now more, now less, according to its needs? 
This is regulated by the medium-sized arteries leading to the parts. In the walls of the small and medium-sized arteries are muscle fibers of a different kind from those of the skeleton. These fibers are spindle-shaped, as shown in Fig. 24, and do not have the cross-markings of the fibers of the skeletal muscles; they are in consequence called Non-striated, Smooth

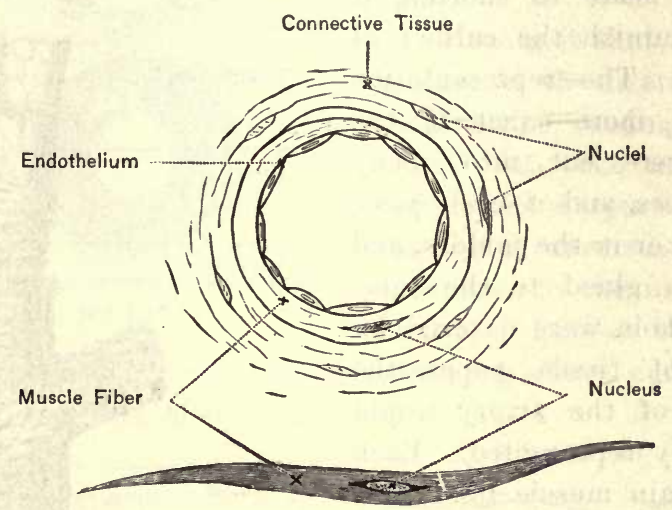

Flg. 25. Plain Muscle Fiber. Isolated and in wall of Artery.

or Plain Muscle Fibers. They are arranged circularly in the walls of the arteries. These fibers have, in common with all muscle fibers, the power of shortening, but at a much slower rate than the striated fibers. When they shorten they reduce the size of the artery, and, therefore, for the time, less blood can flow through the artery. When the muscle fibers cease to shorten, the artery widens, and allows more blood to pass through it.

To illustrate the action of the muscles in the walls of an artery, let the water run through a hose or large rubber tube. Now, if a row of persons take hold of this tube, the grip of their hands is like that of the muscles. When the hands 
tighten their grip, the caliber of the hose or tube is diminished, and less water is allowed to flow through it. When the hands relax, the tube, being elastic, allows more liquid to flow through it.

To represent a small artery, take a small thin-walled rubber tube, and wind a red thread around it. Now, if the thread could be made to shorten, it would diminish the caliber of the tube. The representation would be more exact if the thread were cut into many short pieces, and if each piece were thicker in the middle, and were then glued to the tube. If the whole were covered by a layer of tissue paper the structure of the artery would be roughly represented. Each of the plain muscle fibers has a Nucleus near its center.

These plain muscle fibers are further like the skeletal mus-

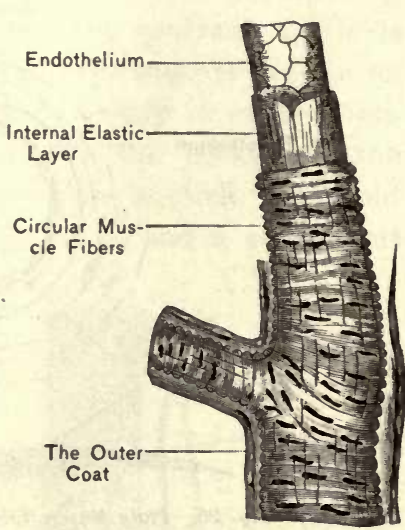

Fig. 26. Coats of a Small Artery. (After Landois \& Stirling.) cles in that they are under the control of the nerves, but they are involuntary in their action. We cannot interfere with the action of these muscles, no matter how strongly we may will to do so. Without our thinking about it, more blood goes to the muscles of the legs when we walk, more to the brain when we are studying, to the digestive organs after eating, etc.

The Blood-Flow in the Capillaries. - The arteries divide and subdivide, and become capillaries, which have connecting branches, forming a close network of tiny thin-walled tubes. These penetrate and pervade nearly every tissue of the body. 
The blood cannot do its work till it gets into the tissues, and to reach the tissues it must soak through the walls of the capillaries. The work of the heart and arteries is to keep a

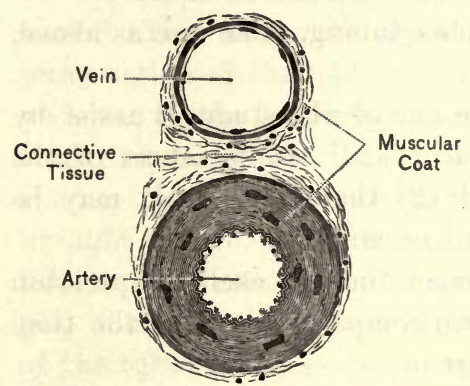

Fig. 27. Cross Section of Small Artery and Vein.

steady flow of blood through the capillaries, that the tissues may be constantly supplied.

How is it that the jerky action of the heart, at each contraction sending a jet of blood into the arteries, shown by a spurt when an artery is severed, and also indicated by the intermittent pulse, - how is this intermittent flow converted into the steady, uniform current that we have seen in the capillaries?

A few experiments may make this matter more clear.

Material:-

1. A common rubber syringe.

2. A glass tube three feet long and seven-sixteenths of an inch outside diameter.

3. Four inches of the same size glass tubing, for making connections.
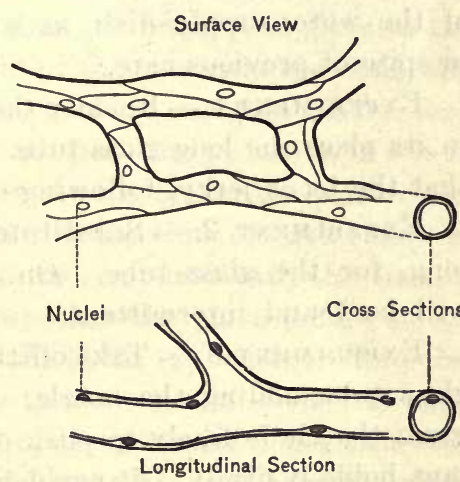

Fig. 28. Capillaries, composed of a single layer of cells.

4. Several nozzles, made of the same size glass tubing, all fine, but of varying degrees of fineness. 
5. India-rubber tubing, twelve feet, three-eighths of an inch inside diameter. This should be black, pure gum, rubber which is more highly elastic than the other kinds.

6. Three feet of rubber tubing, same size as above.

7. Four inches of white rubber tubing, same size as above, for making comnections.

In all the experiments, have one of the students assist by holding the outlet tube, so that (1) all the members of the class may see the stream, and (2) that the stream may be suitably directed, as into a pail or sink.

Count aloud, to mark the exact time of each compression of the bulb, so the students can compare this with the time and duration of the jets of water.

Be very careful to use perfectly clean water, as any fine particles of sediment drawn into the tube are likely to clog the fine outlet of the nozzle. And it is well to take the further precaution not to let the supply tube touch the bottom of the water-supply dish, as some fine sediment may get in in spite of previous care.

Experiment 1. - Remove the nozzle of the syringe, and put in its place the long glass tube. Work the syringe, and note that the jet is jerky, following each contraction of the bulb.

Experiment 2. - Substitute the rubber tube, three feet long, for the glass tube. On working the bulb the stream will be found intermittent.

Experiment 3. - Take off the rubber tube and replace the glass tube, adding the nozzle. Here the pressure will be so great that it is likely to push off the nozzle unless the assistant holds it firmly. It could be tied on, but this takes more time. On working the bulb, greater effort must be made on account of the resistance caused by the narrower outlet.

Experiment 4.-Once more substitute the rubber tube, this time with a glass nozzle in its end. Now, on working 
the bulb, resistance will be felt, and the stream will be constant, or nearly so, and will continue for some time when the bulb is no longer worked. This is, clearly, because the rubber has been stretched, chiefly laterally, and is now "stretching back." That is, by the elastic reaction of the rubber tube the jerky action of the bulb is converted into the steady flow that we see. In the first experiment we had a rigid tube and practically no resistance. In the second, although the tube was elastic, there was no resistance, so the elasticity was not brought into play. In the third, there was resistance, but the tube was inelastic. In the fourth, the resistance brought into play the elasticity of the rubber tube, and the elastic reaction of the tube continues (so to speak) the action of the bulb between two successive strokes. In this experiment the pulse can be felt in the tube.

Experiment 5. - Repeat the last experiment, except with the change of taking the rubber tube ten or twelve feet long. Double this along a table so that the nozzle lies close to the bulb. Let each pupil take hold of the tube near the bend, and then advance toward the bulb, holding one part of the loop in each hand, noting the time at which the pulsation is felt in the two parts of the loop. Let the teacher or the person who works the bulb count aloud to give the time of each compression of thc bulb, so that it can be compared with the time of the pulsations. Observe (1) whether the pulse occurs at the instant of the compression of the bulb; (2) whether the pulse is felt at the same instant by the two hands when one hand holds the tube near the bulb and the other hand near the nozzle. Compare with this the intervals between the heartbeats and the pulse as felt at (1) the temple, (2) the wrist, (3) the ankle.

The pulse in the artery and in the rubber tube must not be confounded with the blood-flow itself. The pulse is a wave 
running much faster than the blood current. To prove this point, make the following experiment: Pour a little red ink into a dish of water, and while working the bulb of the syringe as in Experiment 4, quickly transfer the supply tube to the colored liquid. The pulse will continue unchanged, but it may take several strokes before the colored liquid issues from the nozzle.

The pulse may be compared to the shock that runs through a freight train. When the engine bumps against the first car, this car strikes tho next, and so on through the whole length of the train. The shock has gone the length of the train, but no car has moved more than a few feet.

The Veins. - The capillaries, after penetrating the tissues, reunite to form small veins, till finally two great veins, the Caval Veins, Precaval and Postcaval, return the blood to the heart. The veins, like the arteries, are smooth inside and elastic (though less elastic than the arteries). They are thinner than the arteries, and, in consequence, collapse when the blood flows out of them, whereas the larger arteries stand open, after they are emptied of blood.

The Valves in the Veins. - The only valves in the arteries are those which we have seen at the beginning of the aorta and pulmonary artery. Many of the veins have similar pocket-like valves, though loss strong than those of the arteries. Dissect back the skin from the throat of the cat or the rabbit, till the Jugular veins are well exposed. Let the head of the animal hang over the edge of the table, and observe that as the blood passes toward the head it causes a marked bulging at certain points. With the handle of the scalpel gently stroke the vein toward the head, watching these swellings. Dissect out the vein from the head to the shoulder. Insert the nozzle of a syringe, first into one end, then into the other, and note the effect of sending a stream 
of water in the two directions. Cut the vein open along one side, and pin it to a piece of shingle, with the inner side out. The thin, pocket-like valves ought now to be seen. They are usually in pairs, but sometimes single or in threes. It is important to note that they all have the mouths of the pockets toward the heart, so that the blood flows freely toward the heart, but is prevented from flowing the other way on account of the filling of the valves by the reflow of the blood stream. When the blood is flowing through the veins toward the

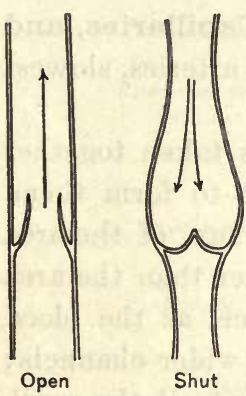

Flg. 29. Venous Valves. heart the valves lie against the walls of the veins.

The valves are most numerous in the medium-sized veins, and especially in the veins of the extremities; more abundant in the leg than in the arm. Valves are absent from the Caval and some other veins, and from the very small veins. While experimenting with the rein, to see the valves and their actions, note also the smoothness of the lining. Test also the elasticity of the veins.

Effect of Pressure on the Veins. - Since the valves in the veins open toward the heart, any intermittent pressure on the veins helps to push the blood on toward the heart. The valves are most numerous in the superficial veins and those of the muscles. The pressure of the muscles during their action (thickening while shortening) produces pressure on the veins; and as the muscles act for a short time only, and then relax, this alternate compression and release aids very considerably in moving the blood on toward the heart. It is worthy of remark that this effect is more pronounced at the time the 
muscles need the most active circulation; namely, when they are in action, and are using the most blood. The heart has power enough to pump the blood clear around from each ventricle to the auricle of the other side of the heart; but this outside aid comes in good play to relieve the heart at a time when it has an unusual amount of work to do, as when one is using a large number of muscles vigorously.

"Every active muscle is a throbbing heart, squeezing its blood tubes empty while in motion, and relaxing so as to allow them to fill up anew."

Rate of Blood-Flow in the Arteries, Capillaries, and Veins. - The blood flows most rapidly in the arteries, slowest in the capillaries. Why is this ?

When an artery divides, the two branches taken together are larger than the one artery that divided to form them. Stated more exactly, we would say that the sum of the area of the cross-sections of the branches is greater than the area of the cross-section before branching. Hence, as the blood flows on, it is continually entering wider and wider channels; and we are told that the united cross-section of all the capillaries fed by the aorta is several hundred times that of the aorta itself.

If we walk along a stream we see that the channel varies considerably in width and depth. Where the channel is large, whether from increased width or depth, there the current is slower; but wherever the channel is reduced, the current is more rapid. So the stream in the relatively narrow artery is swift. In the capillaries, although any individual channel is small, these channels all together are wide; the result. is the same whether a river widens out into a single lake, or divides into a great number of channels running past innumerable islands. All the tissues of the body may be regarded as so many islands lying between the capillary streams. 
When the blood re-collects in the veins it is entering narrower channels, and its rate is quickened; but as the veins are wider than the arteries, the stream does not enter the heart with the velocity with which it left that organ. The veins hold more blood than the arteries, and in dissecting the cat

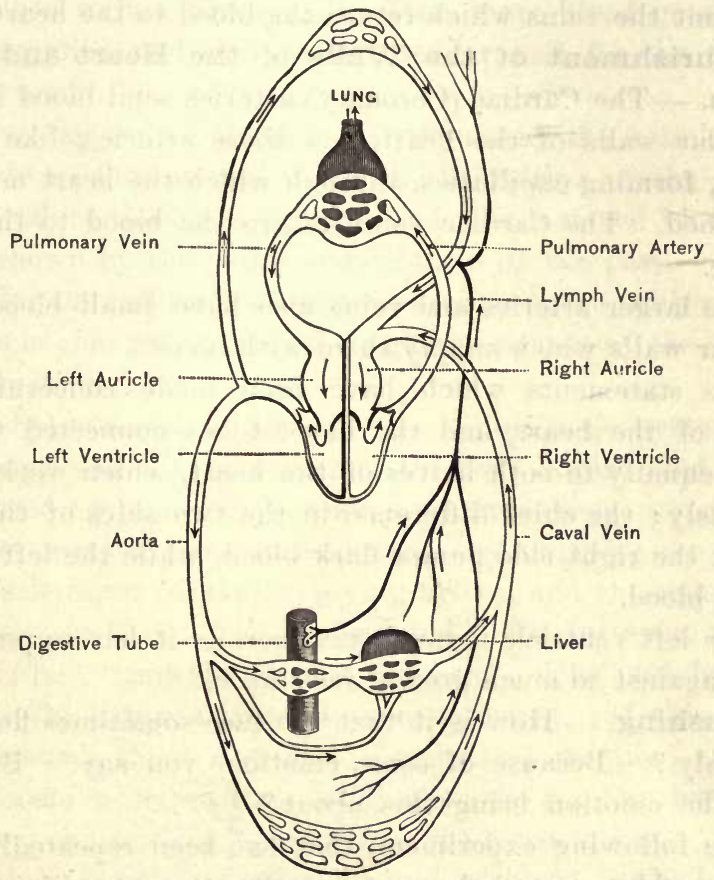

Fig. 30. Plan of Circulation. (Dorsal View.)

or rabbit it will be noticed that the arteries are emptied of blood; that the tissues of most of the organs are fairly free from blood; but that the great veins, such as the caval veins, are full. 
If the blood tubes leaving the heart could all be united, they would be best represented by a funnel with its tube connected with the heart. If another funnel were placed with its mouth to the mouth of the first, their point of union, the widest point, would represent the capillaries; and if the second funnel had a wider tube than the first, it would fairly represent the veins which return the blood to the heart.

Nourishment of the Walls of the Heart and Blood Tubes. - The Cardiac (Coronary) arteries send blood into the muscular walls of the heart, and these arteries, like others, divide, forming capillaries, through which the heart muscle is nourished. The Cardiac veins return the blood to the right auricle.

The larger arteries and veins also have small blood tubes in their walls which supply them with blood.

The statements which have been made concerning the action of the heart, and the blood tubes connected with it, apply equally to both halves of the heart, which work simultaneously; the chief difference in the two sides of the heart is that the right side pumps dark blood, while the left pumps bright blood.

The left ventricle is much stronger, as it has to pump the blood against so much greater resistance.

Blushing. - How is it that the face sometimes flushes so suddenly? Because of some emotion, you say. But how does the emotion bring this about?

The following experiment, that has been repeatedly made on the rabbit, may help us to answer the question. If the sympathetic nerve, in the neck, which sends branches to the head, be cut, the ear of that side at once grows red; i.e., it blushes. But if the end of the nerve connected with the ear be stimulated, the ear becomes pale. We have already learned about the muscles in the wall of the arteries, and their action. 
We are now prepared to understand that in the normal condition nervous impulses are acting on these muscles, keeping them partly shortened, and so keeping the arteries of a moderate size. When the nerve is cut, these nervous impulses are interrupted, and the muscle fibers, being no longer stimulated, relax, and allow the artery to widen, and so permit more blood to flow through it. Thus more blood goes to the parts supplied, and they become redder, and, for the time at least, warmer.

When the end of the nerve is stimulated, the muscle fibers are made to shorten and diminish the caliber of the artery, and to reduce the blood supply; and this lessened blood supply is shown by the pallor and coldness of the part.

Under the influence of varying emotions, the caliber of the arteries is changed, largely through the nerves of the sympathetic system.

In the same way, through the sympathetic system, the blood supply of all the organs of the body is regulated. Any organ needing more blood sends a message (nerve impulse) to some nerve center; and in response nerve impulses are sent to the muscle fibers of the supplying artery, and the amount of blood sent to that organ is regulated. For instance, a piece of ice is laid upon the skin of the hand. The part becomes pale, as the arteries have become narrowed. If this action be continued, there may set in a decided reaction, and the part become more red than usual when the reaction has made the artery wider than it was before the constriction.

As there is only a certain amount of blood in the body, it is evident that if one organ receives an extra supply, some other organ or organs must, for the time, receive less. For instance, one begins to walk vigorously. The large muscles of the lower limbs and trunk become active, and they need more blood. They therefore send messages to some nerve 
center (probably in the spinal cord); and by reflex action the arteries supplying the lower limbs are widened, and these muscles receive more blood. But these muscles make up a very considerable part of the weight and bulk of the body. While in action they take the lion's share of the blood. The brain, at such a time, would receive less; and it would be folly to expect the brain to work at its full capacity while the blood was called away to other organs.

When we exercise vigorously the heart beats faster, and this of itself would tend to increase the blood supply to all organs. But this mechanism of widening the channel leading to the working organs, while the arteries to the other organs are made smaller, or at least are not enlarged, solves the problem of supplying each part according to a greatly varying need, while not sending too much to a part not needing it.

When we sit quietly intent upon study, the brain has a larger supply of blood, and other parts now have less; in such condition the feet may easily grow cold.

If we wish to draw away some of the blood from the brain after prolonged study, we take a little gentle exercise to better prepare for sleep.

Dissection of the Sympathetic Nervous System. - In a cat or a rabbit open the chest cavity, and pull the heart and lungs to one side. Close to the spinal column there may be seen a white nerve with a ganglion for every rib. This is the 


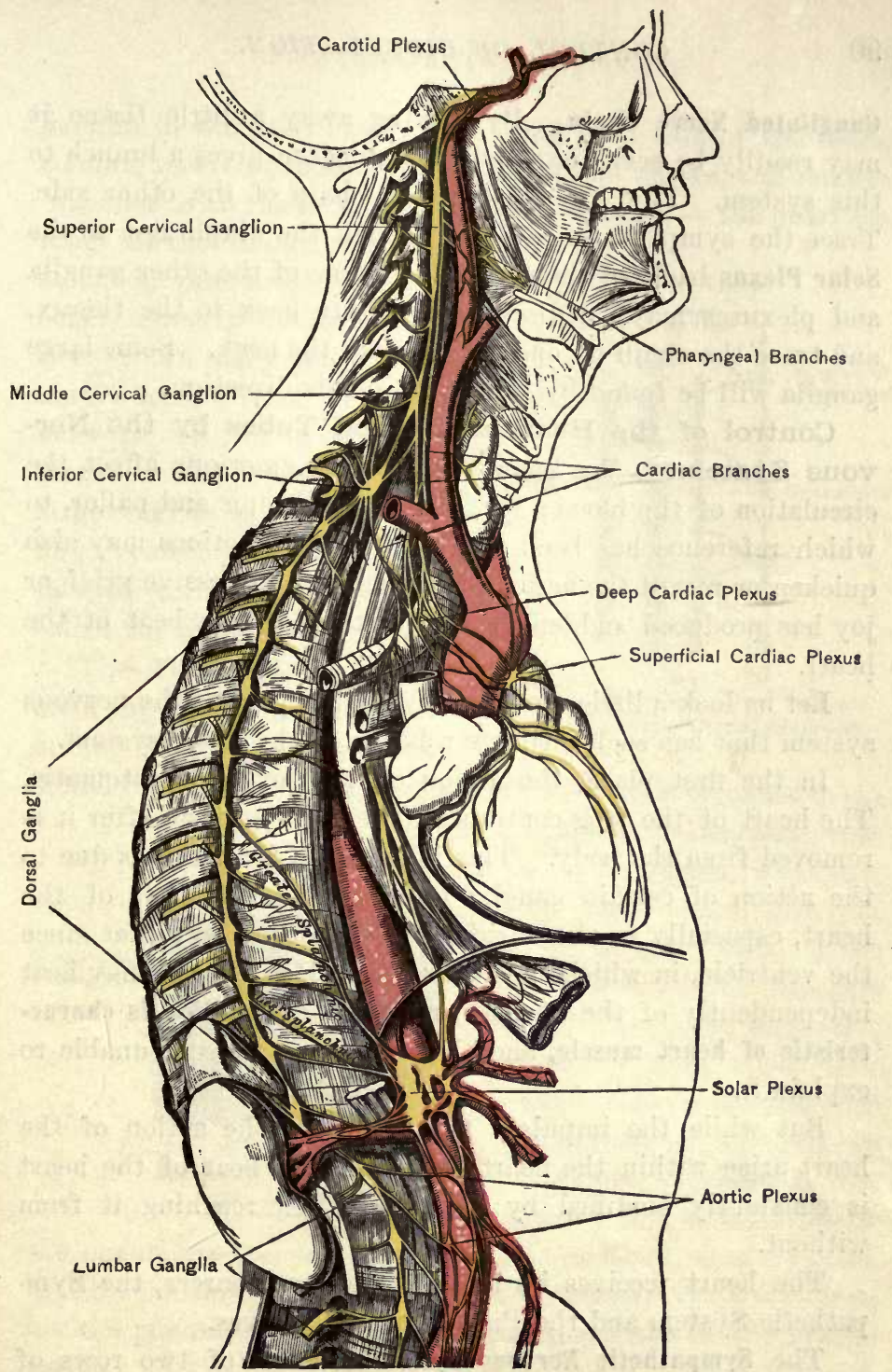

Flg. 32. Vertical Section of Body, showing Sympathetic Nerves and Ganglia of Right Side and their Connection with the Cerebro-Spinal Nerves. 
Gangliated Nerve Chain. By picking away a little tissue it may readily be seen that each spinal nerve gives a branch to this system. Find the sympathetic chain of the other side. Trace the sympathetic system through the diaphragm to the Solar Plexus back of the stomach. Some of the other ganglia and plexuses may be discovered. Turn back to the thorax, and trace the chain on one side up into the neck. Some large ganglia will be found by following in this direction.

Control of the Heart and Blood Tubes by the Nervous System. - We know that certain emotions affect the circulation of the blood; for instance, blushing and pallor, to which reference has been made. Certain emotions may also quicken or retard the action of the heart. Excessive grief or joy has produced sudden death by stopping the beat of the heart.

Let us look a little more closely at that part of the nervous system that has such intimate relation to the blood system.

In the first place, the action of the heart is automatic. The heart of the frog continues to beat a long time after it is removed from the body. This is regarded by many as due to the action of certain ganglia imbedded in the walls of the heart, especially in the auricles; while others say that since the ventricle, in which no ganglia have been found, may beat independently of the auricles, rhythmic contraction is characteristic of heart musele, and that we are at present unable to explain it.

But while the impulses that originate the action of the heart arise within the heart itself, still the beat of the heart is constantly modified by nerve impulses reaching it from without.

The heart receives its nerves from two sources, the Sympathetic System and the Pneumogastric Nerves.

The Sympathetic Nervous System consists of two rows of 
ganglia in the body cavity, one along each side of the spinal column, receiving branches from the spinal nerves, and sending branches to all the internal organs of the body, - the heart and lungs in the thorax, and the stomach, intestines, and the other organs of the abdominal cavity. In many places these nerves form a thick network called a plexus. One very large plexus is on the posterior surface of the stomach, and is called the Solar Plexus.

The Pneumogastric Nerves are a pair of the cranial nerves arising from the sides of the spinal bulb; and, passing downward, they give branches to the pharynx, gullet, and stom-

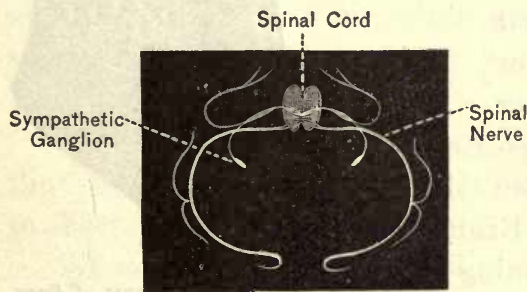

Fig. 34. Ideal Cross Section of the Nervous System. (After Landois \& Stirling.)

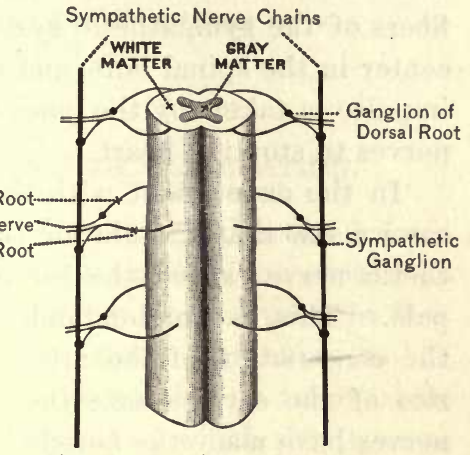

Fig. 33. Relation of Spinal Cord and Sympathetic Nervous System(Diagram). ach, the larynx, windpipe, and lungs, and the heart. Now, whatever other function the pneumogastric nerves may have, they seem to have the power of retarding, or stopping altogether, the beat of the heart; and stimulation of the pneumogastric nerves may make the heart pause in a relaxed condition. Other nerves may quicken the heart-beat, but the pneumogastrics are regarded as a brake on the heart's action. 
This is a case of Inhibition. It is well known that a severe blow over the stomach may cause one to faint by stopping the heart. This is due to Reflex Inhibition of the heart. The blow sends a nerve impulse by fibers of the sympathetic system to the center in the spinal bulb, and thence an impulse is taken by the pneumogastric nerves to stop the heart.

In the experiment with the rabbit's ear we saw that stimulating the sympathetic nerve caused the ear to become pale. This, we understand, is due to the constriction of the arteries of the ear, because the nerves have made the muscle fibers of these arteries shorten. Such nerve fibers are called Constrictors, or Vasoconstrictors. They run in the sympathetic nerve, but have their origin and center in the spinal bulb. Other fibers may cause the opposite effect, namely, dilation, and are therefore called Vaso-dilators. Examples of these may be found running to the arteries of the limbs. We have seen

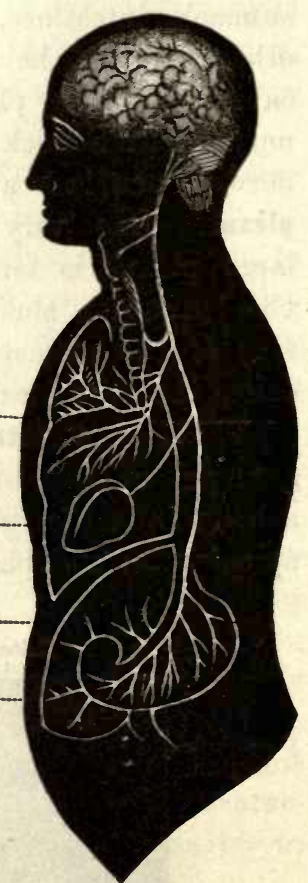

Flg. 35. Diagram of Pneumogastric, Nerue. that when the muscles of any organ, say the legs, act, they need a greater supply of blood. Now, at the same time that nerve impulses are sent to the muscles of the legs to make the muscles shorten, impulses are sent along other fibers of the same nerves to make the arteries dilate, and allow more blood to flow to these muscles. 
The vaso-constrictor nerves and the vaso-dilator nerves taken together are called Vaso-motor nerves.

There is no evidence that the sympathetic ganglia are centers of reflex action.

Let us be alert to discover the action of these nerves as we study other parts of the body.

\section{EFFECTS OF ALCOHOL ON THE CIRCULATION.}

"Alcohol stimulates the heart, producing increased force and rapidity of the cardiac beat. It thus tends to increase the blood-pressure by acting on the heart, and to increase the flow of blood from the arteries into the veins. The effect on the blood-pressure is, however, partly counteracted by a coincident dilatation of the blood-vessels of the skin, which thus become flushed, and tends to produce more sensible perspiration." Treatise on Hygiene, Stevenson and Murphy.

"The warm and flushed condition of the skin which follows the drinking of alcoholic fluids is probably, in a similar manner, the result of an inhibition of that part of the vasomotor center which governs the cutaneous arteries." - Text Book of Physiology, Foster.

The control of the muscles in the walls of the arteries being thus interfered with, the circular muscles are no longer made to shorten, and the artery dilates, and receives more blood.

We may thus account for the flushing of the skin of the face, which in many individuals quickly betrays indulgence in alcoholic drink. If this flushing is too often repeated, the arteries gradually "lose tone," and the condition becomes permanent. The circulation in the whites of the eyes may be affected, making them "bloodshot."

Similar congestion occurs in the mucous membrane of the stomach from the presence of alcohol, which may become a 
permanent inflammation, followed in time by very extensive changes in appearance and function. It is said that most of the alcohol swallowed is absorbed directly from the stomach, and hence the intestines are not so directly affected.

Virchow says, "Alcohol poisons the blood, arrests the development of the corpuscles, and hastens their decay." - "It is said to change the form, lessen the number, and diminish the size of the corpuscles. A degenerate or ill-conditioned blood, circulating in all parts of the system, induces abnormal nutrition. Abnormal nutrition is always followed by abnormal or perverted actions, physical, mental, and moral."-CuTter.

The Blood. - To get a drop of blood from the finger, wind a cord around the finger, beginning at the base, drawing the cord moderately tight, until the last joint is reached. By this time the end of the finger is usually well distended with blood. With a clean needle make a quick, sharp, light puncture near the base of the nail; this ordinarily brings a small amount of blood. Put a small drop on each of several slides and quickly cover with coverslips. Examine with a high power.

1. The Colored Corpuseles. These are often called the Red Corpuscles. But while in the mass they give the blood a red appearance, individually they are faint yellowish red. In shape they are seen to be circular disks, hollowed on each side like a sunken biscuit. As they are hollowed on both sides they are more accurately described as biconcave. These corpuscles tend to gather side by side, in rolls, like coins. They have no nuclei.

2 . In the open spaces between the rolls of colored corpuscles may occasionally be found some of the spherical corpuscles. They are usually called the White Corpuscles, but are better designated as the Colorless Corpuscles, since the others have only a slight color, and these have none. They usually 
have a dotted appearance. It is not so easy to distinguish the two kinds of corpuscles as in the case of the frog's blood, for the two kinds are more nearly of the same size in the human blood; and, further, when the colored corpuscles of human blood are seen flatwise they present a circular out-

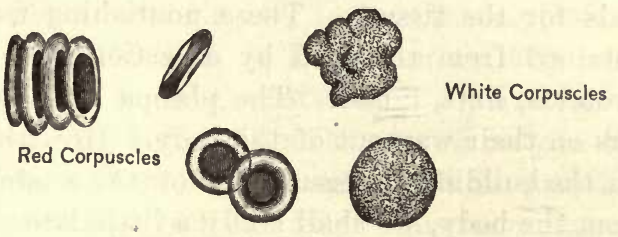

HIGHLY MAGNIFIED

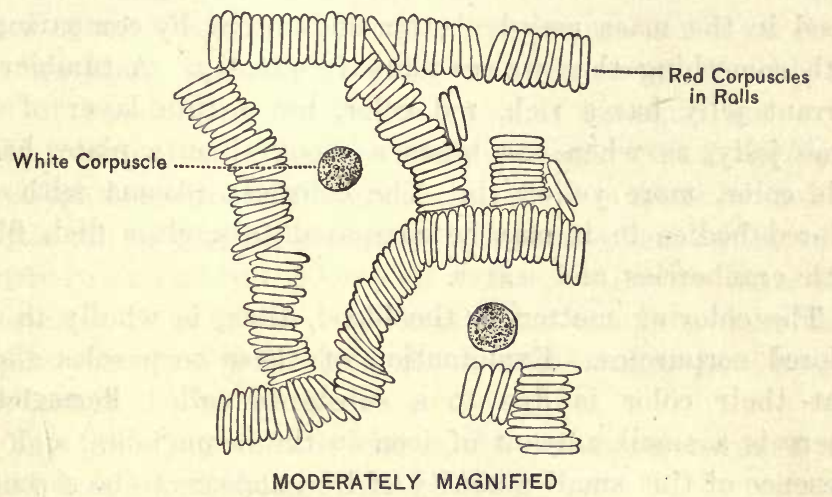

Fig. 36. Red and White Corpuscles of the Blood.

line; while the frog's colored corpuscles are elliptical. But with a little study the two may be distinguished. As in the frog's blood, the colorless corpuscles have ameboid movements, though they are not very marked unless the blood be warmed to about the temperature of the human body.

The blood is composed of a clear liquid, the Plasma, and 
the Corpuseles. In the drop of blood examined under the microscope, the Plasma occupies the clear spaces between the corpuscles. The corpuscles make up one-third of the bulk of the blood, and the plasma the other two-thirds. The plasma consists chiefly of water, having in solution various salts, including common salt; it also contains the nourishing materials for the tissues. These nourishing materials have been obtained from the food by digestion, and consist chiefly of Proteids, Fats, Sugar. The plasma also contains waste matters on their way out of the body. How the food is prepared for the building of tissue, and how the waste matter is removed from the body, we shall study a little later.

The difference in color of an individual corpuscle and the blood in the mass may be better understood by comparing it with something that we see more frequently. A tumbler of currant jelly has a rich, red color, but a thin layer of the same jelly, as when one takes a spoonful on a plate, has a pale color, more yellowish. The colorless plasma with the colored bodies in it may be compared to a glass dish filled with cranberries and water.

The coloring matter in the blood, then, is wholly in the colored corpuscles. Examination of these corpuscles shows that their color is due to a substance called Hemoglobin. There is a small amount of iron in the hemoglobin, and the presence of this small quantity of iron appears to be essential to give the blood its color. When we come to the study of respiration we shall see that the hemoglobin in the corpuscles is the chief agent in picking up the oxygen from the air in the lungs and carrying it to the tissues in the body.

It will be well here to examine again the flow of blood in the capillaries of the frog's web. It will be seen occasionally that when one of the colored corpuscles is pressed against an angle at the forking of the blood stream, it is sometimes bent, 
and that as soon as the pressure is discontinued the corpuscle springs back to its former shape, showing that it is elastic.

The Coagulation of Blood. - When the blood escapes from its natural channels it usually changes from a liquid to a jelly-like condition. This is known as Coagulation. It is due to the formation of the threads of Fibrin from the plasma. These threads of fibrin entangle and inclose the corpuscles, and the two constitute the Clot, or Coagulum as it is more technically termed. The liquid that afterward separates from the clot is the Serum, and differs from the plasma only in the removal of the fibrin, which is exceedingly small in quantity, though of great importance in its action. Many experiments have been made, and much has been written about the coagulation of the blood, and perhaps its real cause is not yet clear. But we know that the coagulation often serves to stop the flow of blood from wounds, and this is its main use.

If freshly drawn blood be stirred rapidly with a bundle of wires (perhaps the most convenient stirrer is made by making a little roll of wire screen), there will soon collect on the wires a stringy substance. Thorough washing will soon leave this colorless. It is Fibrin. If the stirring has been done thoroughly the blood will no longer clot, no matter how long it may stand.

The following scheme shows the difference between the liquid blood and the coagulated blood:-

Liquid Blood $\left\{\begin{array}{l}\text { Plasma } \ldots .\left\{\begin{array}{l}\text { Serum } \\ \text { Fibrin } \\ \text { Corpuscles.......... }\end{array}\right\} \text { Clot } \ldots\end{array}\right\}$ Coagulated Blood.

Amount and Distribution of the Blood. - The blood constitutes about one-thirteenth of the weight of the body. It is alkaline in reaction, and is a little heavier than water. 
The blood is divided among the organs about as follows:-

1. One fourth is in the heart, arteries, and veins (including those of the lungs).

2. One fourth in the liver.

3. One fourth in the skeletal muscles.

4. One fourth in the other organs.

The Lymph. - We have seen that the capillaries have very thin walls. Through their walls part of the plasma of the blood soaks out, and is then called Lymph. It gets into irregular cavities in the tissues called Lymph Cavities, or Lymph Spaces. Most of these lymph spaces are minute chinks or crevices in the connective tissue of the different parts of the body. Opening out of the lymph spaces are irregular passageways called Lymph Capillaries, and these lymph capillaries are continuous with thin-walled tubes called Lymph Vessels (they would better be called Lymph Tubes, for the same reason that we prefer the term "blood tube" to "blood vessel," because the expression "vessel" is naturally misleading). These lymph tubes might be called the Lymph Veins, since they join still larger tubes, closely set with valves, similar to those of the veins. But, unlike the blood veins, the lymph veins do not gradually increase in size by confluence. They suddenly form a large tube, the Receptacle of the Chyle, beginning in the upper part of the abdomen. This tube soon narrows and passes through the diaphragm, close to the spinal column, and up along the column near the aorta, and empties into the veins of the neck at the junction of the left jugular and left subclavian veins. This tube is the Thoracic Duct, but would better be called the Lymph Duct. It has numerous valves, and, like some of the smaller lymph veins, it presents a beaded appearance, due to the filling and bulging out of the valves. In the right side of the neck is a short Right Lymph Duct which receives lymph from the right side of the head, neck, and thorax, and from the right arm. 
In dissecting the frog, the looseness of the skin is very noticeable. The large spaces under the skin are lymph spaces. Sometimes considerable lymph is found here, so that in holding up a frog the sagging of the skin from the weight of the lymph may be easily seen.

There are valves where these lymph ducts empty into the veins, which prevent any reflow of liquid into the ducts, but allow the lymph to pass freely into the veins. There are plain muscle fibers in the walls of the lymph ducts. It will now be seen that while the blood leaves the left ventricle by one tube, the aorta, it returns to the right auricle, not merely by the two eaval veins, but that a part of the blood (i.e., of the liquid part of it) does not return by blood veins at all, but having left the blood system proper through the thin walls of the capillaries, it is brought back to the heart by the lymph veins, which, however, join the blood veins just before they empty into the heart. There is, in other words, only one set of distributing tubes, but there are two sets of collecting or returning tubes. (See Fig. 37.)

In its course the lymph passes through many kernel-like masses, the Lymphatic Glands. Lymph contains corpuscles which are considered identical with the colorless blood corpuscles. It is thought that these corpuscles may be formed in the lymphatic glands.

The flow of lymph is partly due to the blood pressure in the capillaries; this pressure is caused by the heart. (In the frog there are two small hearts, - not, however, near the bloodpumping heart, - and these pump the lymph along.) In our bodies the flow of lymph is largely aided by any pressure that may be brought to bear on the lymph veins; for, on account of the valves, as in the blood veins, any pressure must push the liquid toward the heart. Thus the action of the muscles in the limbs, and in the chest and abdomen, the movements of 
breathing, and in the bending of the body, etc., all help in this flow, which is always, probably, very much slower than that in the blood veins.

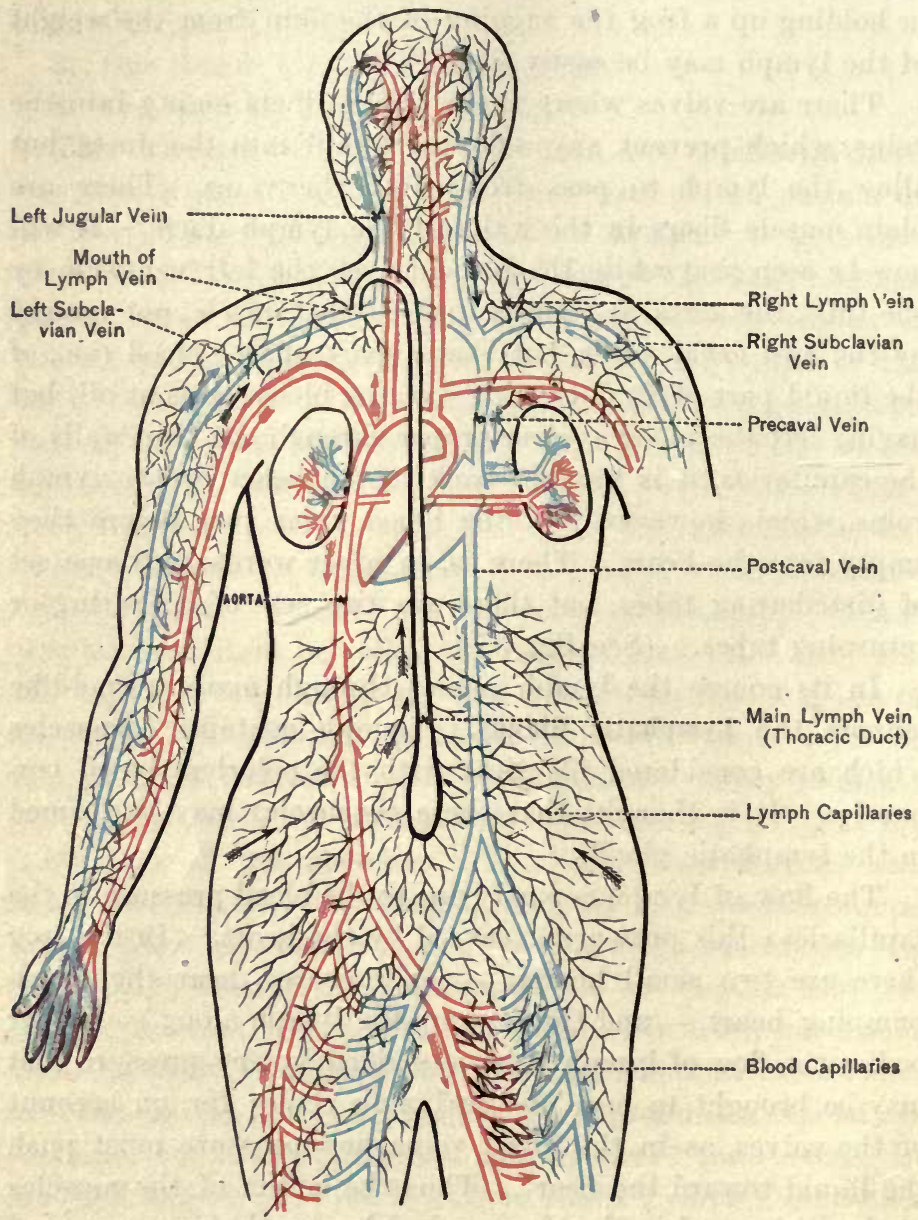

Fig. 37. Dlagram of the Clrculation of Blood and Lymph. (Dorsal Vlew.) 
Lymph is a clear liquid. (Chyle and the lacteals will be considered when we study digestion.) It is more watery than the blood plasma, but contains a share of all its nutritious substances. Lymph may. be defined as diluted blood minus red corpuscles. The blood proper never reaches the tissues. The cells of the tissues are bathed in the lymph which fills the spaces in the connective tissue (and we have seen that the connective tissue pervades nearly all the tissues of the body),

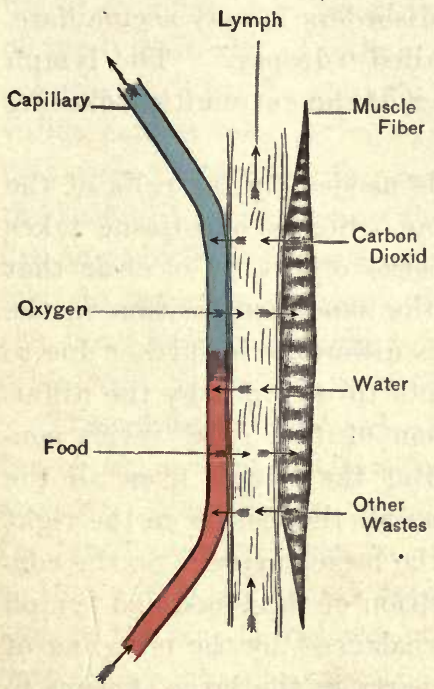

Fig. 38. Relation of Blood and Muscle.

(Lymph being middleman.) as water may fill the spaces left between stones built into a wall. The cells get all their nourishment from the lymph, and into the lymph they throw all their waste matter. Each cell may be compared to an individual ameba, which lives in water, and takes all its nourishment from that water, and throws all its waste product into the same water. As water is the medium in which the ameba lives, so we may say. lymph is the medium in which the cells of the body live.

The cells of the body, i.e., all the active, working cells, may, therefore, be said to live an aquatic life, and only dead cells, as of hair, epidermis, etc., are in air. We might also say that not only the human body, but all animal life, is aquatic.

We can see that the movement and renewal of the lymph 
is as necessary as the circulation of the blood itself; is, in fact, the most important part of it.

We have noticed the pericardial liquid. There is also a small quantity of similar liquid around the lungs in the pleural cavities, and in the abdominal or Peritoneal Cavity, around the digestive organs; also in the cavities of the brain. The liquid in each case is lymph; and these cavities, often called Serous Cavities, are Lymph Cavities. They communicate with the lymph tubes. In health the amount of the liquid in these cavities is small, but in certain disorders it may accumulate. In general, such affections are called "dropsy." The lymph may also accumulate in the tissues of the extremities, causing swelling of the limbs.

It is evident that the materials needed by the cells of the different tissues are not the same. So, as one tissue takes certain materials and another tissue others, it is clear that the lymph will not be of quite the same composition in the different parts of the body. This difference is further due to the difference in the waste products thrown out by the different cells. Hence the composition of the blood varies considerably in different regions. But the lymph from all the tissues unites with the blood from all the tissues in the right heart, and on their way to it in the larger veins. So the constant slight differences in composition of the blood and lymph in the various tissues are counterbalanced by the mingling of the currents from these various parts in the large streams to and from the heart that we call the arteries and veins.

The Spleen. - The function, or functions, of the spleen are not well understood. It is believed to have something to do with the renovation of the blood, perhaps forming colorless corpuscles and destroying colored corpuscles. At any rate, the physiologists generally call it a blood gland. It is unlike true glands in that it has no duct, and forms no secretion to 
be poured into any cavity, like the glands of excretion and secretion. It has been found, in the case of accidents to man: and by experiment on the lower animals, that life may continue after this organ has been removed.

Massage. - A system of pressing, rubbing, and kneading the muscles is known as massage. It helps the flow of the blood and lymph, thus aiding in washing out the waste products from the muscles and other parts of the body that are to be reached by pressure. We have seen that one of the benefits of exercise is to promote the circulation of the blood and of the lymph, and so to help get rid of the waste matters that are produced by the activity of the various organs. Many invalids cannot take active exercise. So this passive exercise may very fairly take its place, and assist in the nutrition of the tissues by accelerating the flow of blood and lymph, bringing new nourishment and carrying away wastes. For students who do not take sufficient exercise it is a good thing to rub the body thoroughly and briskly, not only after a bath, but often with the hands or with a dry towel.

Transfusion of Blood.-Transfusion of blood is the transfer of blood from the blood-vessels of one animal to those of another. Transfusion may be direct or immediate, as when the blood-vessels of the two animals are connected by tubing, so that the blood passes from one to the other without exposure to the air; in indirect or mediate transfusion, the blood is first drawn into a receptacle. In indirect tranfusion, the blood is often defibrinated before transference. The blood may be introduced either into an artery or a vein; if into a vein, it is sent in the direction of the natural flow, i.e., toward the heart; if into an artery, in either direction. Soon after the discovery of the circulation of the blood, the operation of transfusion began to be practiced, and high hopes were indulged in as to its value. But it was soon found to be attended by so much 
danger that it is now seldom used. It is resorted to (1) after great loss of blood; (2), after some forms of poisoning, part of the blood is withdrawn and replaced by fresh blood; and (3), in certain disordered conditions of the blood. The chief dangers are, (1), the introduction of air, which forms minute bubbles, and stops the blood-flow in the capillaries; (2), the introduction sometimes causes coagulation within the bloodvessels, and (3), the serum of the introduced blood sometimes destroys the corpuseles of the blood to which it is added. In the earlier practice, lamb's blood was employed; but now, when transfusion is practiced on man, only human blood is used. It has been found safer and better, after great loss of blood from hemorrhage, to introduce a salt solution of about the natural degree of saltness of the blood; this restores the normal volume of circulating liquid, and avoids most-of the dangers except that of introducing air. The numerous fatal results of this operation have shown that it should not be resorted to except in cases of extreme necessity.

For directions about stopping the flow of blood from wounds see chapter XI. and the books named below.

What other process keeps pace with the coursing of the blood through the body, being its running mate, so to speak?

ReAding. - Prompt Aid to the Injured, Doty; Emergencies, Dulles; Emergencies, Howe; First Aid to the Injured, Lawless; First Aid to the Injured, Morton; First Aid in Illness and Injury, Pilcher; Sickness and Accidents, Curran. 


\section{CHAPTER V. \\ RESPIRATION.}

Is it not a very striking fact that we take one breath for every four heart-beats? That whatever quickens the breathing also quickens the heart, so that the two always keep in almost the same ratio? Let us learn what are the many intimate relations of the blood pump and the air pump, the blood system and the air system, of Circulation and Respiration.

The Organs of Respiration are :-

1. The Lungs and Air Tubes.

2. The structures which increase and diminish the size of the chest, principally the diaphragm, and the muscles acting on the ribs.

The lungs consist of :-

1. An immense number of small sacs, the Air Vesicles, which communicate with the

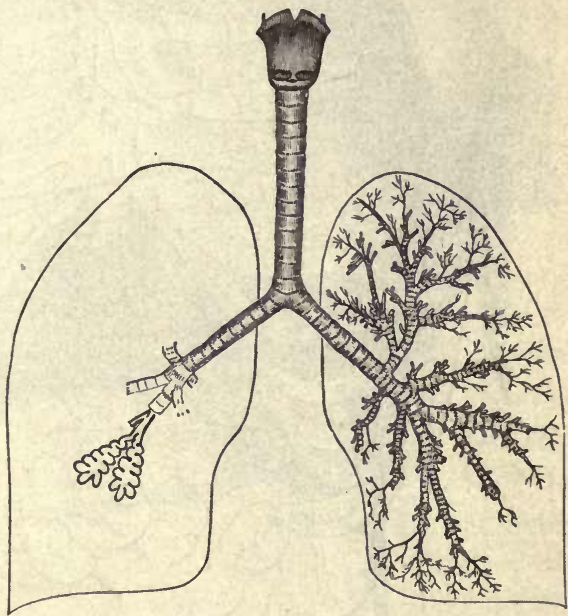

Fig. 39. The Trachea and Bronchial Tubes, Showing Two Clusters (Alveoli) of Air Vesicles. outer air by the Bronchial Twigs, Bronchi, and Trachea.

2. The Pulmonary Capillaries, forming a thick network 105 
around and between the air sacs. These capillaries receive their blood from the pulmonary artery, and return it to the heart by the pulmonary veins.

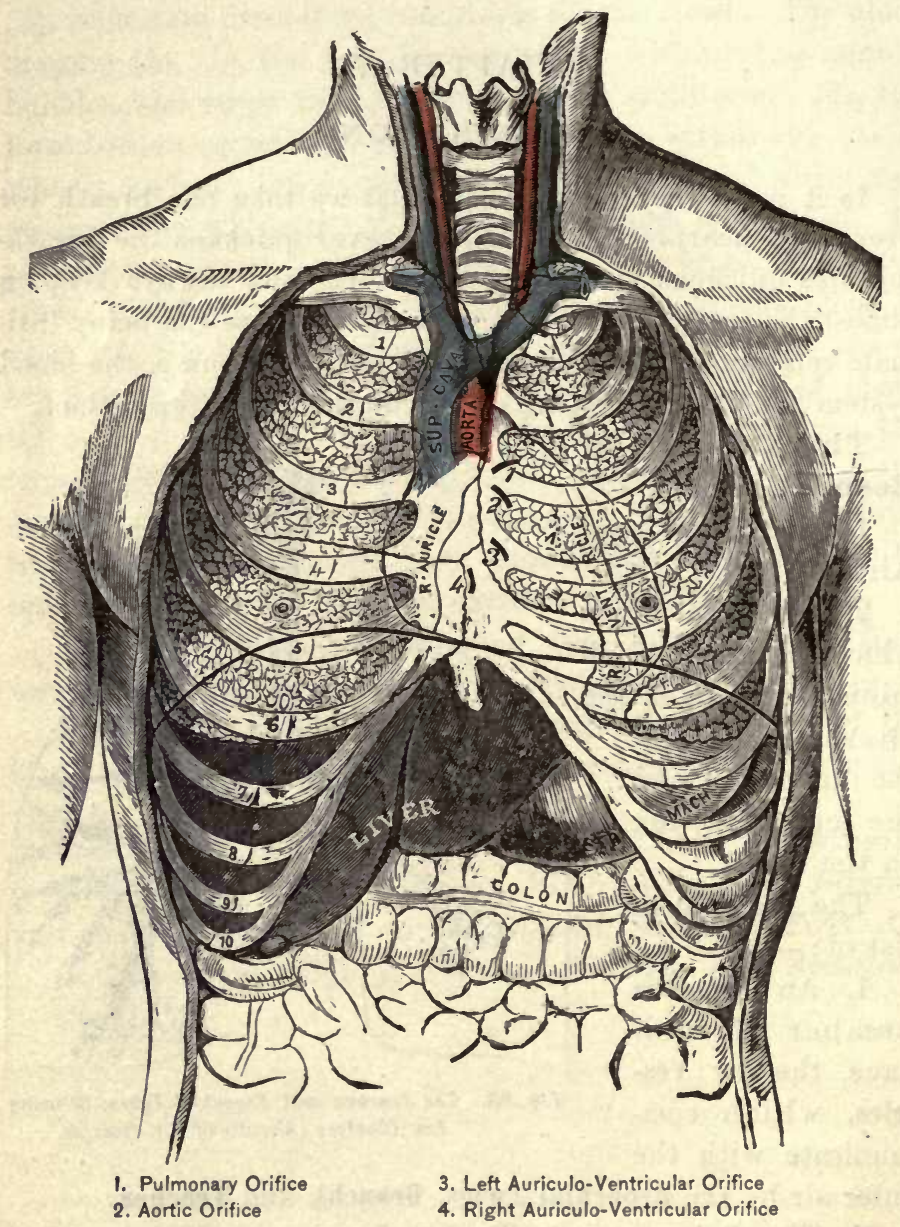

Flg. 40. Front View of the Thorax. The Ribs and Sternum are Represented in Relation to the Lungs, Heart, and other Internal Organs. 
The air vesicles, with their supplying air tubes and their surrounding blood tubes, are bound together by elastic tissue, which fills up most of the intervening space.

The windpipe has in its walls C-shaped cartilages, with the open part of the $\mathrm{C}$ on the dorsal surface. These cartilages continue in the bronchi, and so on until in the smaller twigs they finally disappear.

The cartilages are held together, and the dorsal gap of the cartilages (the gap would be like that of a series of horseshoes piled on top of each other) bridged, by tough fibrous tissue, with much elastic tissue, and with plain muscle fibers; these last-mentioned structures are very abundant in the smaller air tubes.

The lining of the trachea is a Mucous Membrane. It pours out on its surface a substance somewhat like white-of-egg, called Mucus.

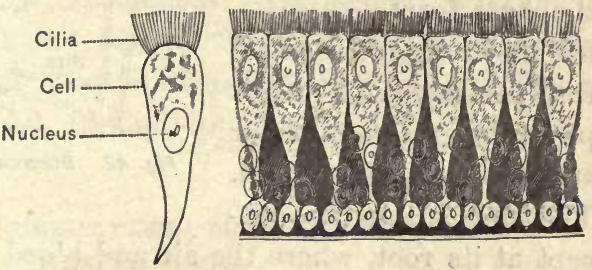

Eig. 41. Ciliated Cells Lining the Air Tubes. This keeps the air moist, and catches particles of dust, etc., that are in the inspired air. There is a' constant slow current of mucus toward the throat, whence it is, from time to time, hawked up. This current of mucus is caused by the Cilia projecting from the lining cells of th 3 trachea. They are little hairlike projections, in countless numbers, like a field of grass, each stalk having the power of bending back and forth, making a quick stroke toward the throat, then a slower recover stroke. Thus the united wavelike action of the myriads of lashing cilia paddles the mucus headward. (See page 388.)

All the cavities and passages in the body to which the air 
has access, such as the digestive and respiratory passages, etc., are lined by mucous membrane (not all ciliated).

The outside of each lung is covered by a thin adherent membrane, the pleura, which completely invests it, except at the root of the lung, where the bronchus and blood tubes enter. Here the pleuraturns toward and adheres to the inner wall of the chest, forming its lining (still called the pleura), and below passes over the anterior surface of the diaphragm. (Exaggerated) The lung is thus free, except at its root, where the air and blood tubes enter. A very small quantity of liquid moistens the contiguous surfaces of the pleuræ on the outside of the lung and the inside of the chest wall, so they move easily one upon the other during respiration. As the lungs are always distended enough to fill the chest cavity, these two surfaces are always in contact. In pleurisy (inflammation of the pleuræ), pain is felt in breathing from friction or adhesion of these surfaces.

In studying respiration, let us constantly keep in mind these facts :-

1. The lungs are highly elastic, and

2. Highly porous, each air vesicle being in direct communication with the outer air by means of :-

3. Air tubes that always stand open, 
4. And are always moist internally.

5. The pulmonary capillaries closely invest each air vesicle.

6. The lungs are always expanded enough to fill all the space in the chest not occupied by other organs, and

7. Freely movable, except at the place of entrance of the bronchi and blood tubes.

8. 'The smooth, moist pleuræ.

The diaphragm is a thin muscle making a complete partition between the abdominal cavity and the chest cavity. It is convex anteriorly, concave posteriorly; its ventral border is attached to the inside of the chest wall about opposite the lower end of the breast-bone, thence obliquely along the border of the ribs (as felt in front), and the dorsal attachment is posterior to the ventral attachment. Its general position is shown in the ventral and lateral views, showing the action of the diaphragm and chest wall during inspiration and expiration. (See Figs. 40, 42, and 45.)

\section{APPARATUS ILUSTRATING THE MOVEMENTS OF RESPIRATION.}

To Show the Action of the Diaphragm and I,ungs. Material. - Bell jar with stopper, sheet of rubber large enough to cover the mouth of the jar, toy rubber balloon, cork (rubber preferred), glass tube, strong rubber band (such as boys use for slung-shots), marble.

Preparation. - Lay the marble on the center of the sheet of rubber, double the rubber over it, stretching the rubber strongly over the marble, and tie the marble firmly in its place. Stretch the sheet of rubber over the mouth of the jar, with the projection made by the marble on the outside, and fasten with rubber band. Bore a hole in the cork, and fix the glass tube snugly in it, so that the lower end of the tube will 
extend about half-way down the jar. Tie the balloon on the lower end of the glass tube.

Experiment 1. - Inflate the balloon. Consider that it requires some expenditure of energy to do this. When the

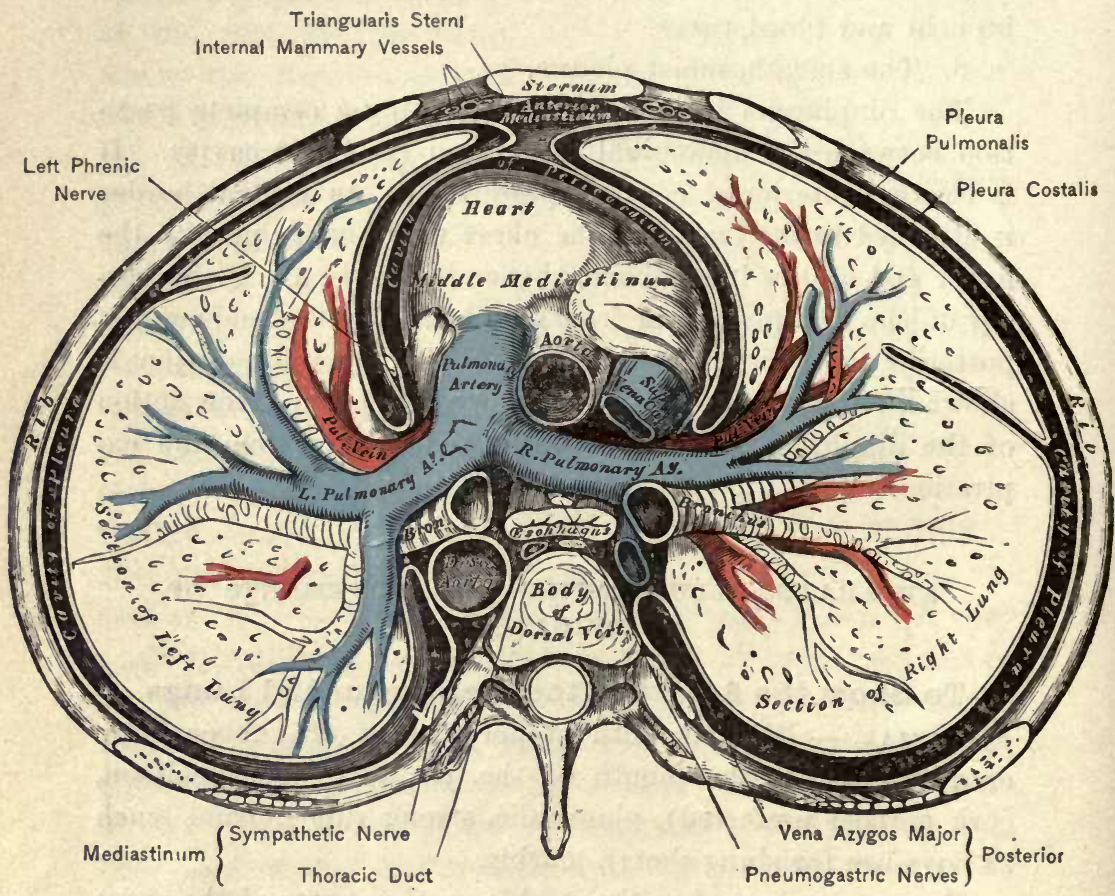

Fig. 43. A Transuerse Section of the Thorax, Showing the Relative Position of the Viscera and Reflections of the Pleurce.

mouth is taken away from the tube the balloon immediately collapses.

Experiment 2. - Insert the balloon and tube into the jar, but do not cork, and repeat Experiment 1. The same results 
as before are noticed, and it will further be seen, or rather heard and felt, that when the balloon is inflated, some air comes out of the jar around the tube, and when the balloon collapses air again enters the jar.

Experiment 3. - Again inflate the balloon, and while it is inflated tightly cork the jar. If all the parts fit well, the balloon should now remain inflated. This may at first seem strange, as the mouth is taken away from the tube, and the tube left entirely open to the air. But it will be seen that to just the extent that the balloon contracts, so much more space is left in the jar outside the balloon. This means diminished pressure; and the pressure of the outer air presses the diaphragm up, and keeps the balloon partly distended, maintaining equilibrium.

Experiment 4. - Pull the diaphragm down, using the marble as a handle. This shows the expansion of the lung by the pressure of the external air when more space is given by the depression of the diaphragm. On releasing the diaphragm, it springs upward, and the balloon becomes reduced in size, driving out part of the air that was in it. This shows how expiration is accomplished, so far as the diaphragm is concerned.

If a bell jar be not at hand, a quart bottle may be used, after cutting off the bottom, as follows: File a deep notch across near the bottom; heat an iron rod, and apply the end of it to one end of the notch, and slowly draw the rod around to the other end of the notch (the rod may need to be reheated). After cracking off the bottom of the jar, file the edges so they will not cut the rubber.

Let each pupil make a drawing, showing the position of the parts in Inspiration and in Expiration.

Action of the Walls of the Chest. - To show the action of the chest walls, take a pair of bellows, and stop the 
hole in the side. Now, when the handles are separated, air enters the nozzle, and when the handles are brought together air is driven out. If a rubber balloon be tied to a glass tube tightly corked in the nozzle of the bellows, and then the bellows are worked, air will enter and inflate the balloon when the handles are separated, and air will rush out when the handles are brought together. If the sides of the bellows have a tight window, the changes in the balloon can be seen.

To illustrate the minute anatomy of the lung, take a rubber balloon, a glass tube, two rubber tubes, one dyed red, the other blue, a bag of netting, with one side dyed red and the other

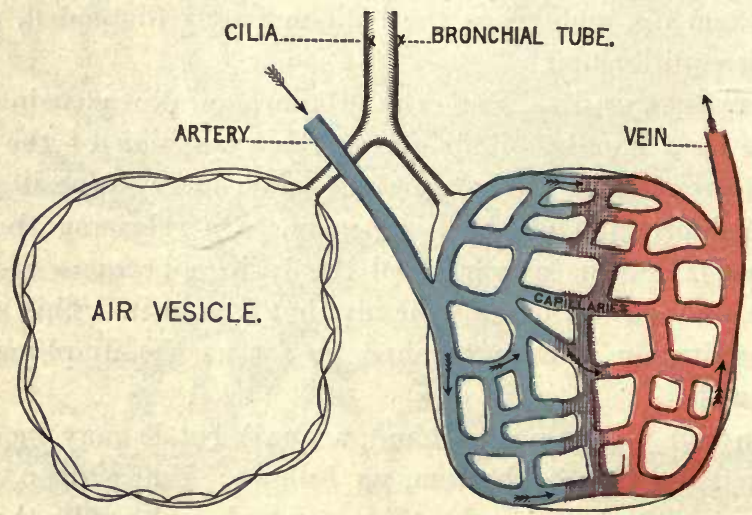

Fig. 44. Minute Structure of the Lungs, Showing Air Vesicles and capillaries.

side blue. Tie the balloon on the end of the glass tube, slip the bag of netting over the balloon, and tie it, with the ends of the rubber tubes on the corresponding sides of the bag. Slip a short piece of the rubber tube on the end of the glass tube, and when the balloon is inflated shut the air in by means of a pinchcock. The balloon represents an air vesicle, the glass 
tube a bronchial twig, the blue tube a subdivision of the pulmonary artery, the netting the capillaries around the vesicle, and the red tube one of the branches of the pulmonary veins.

That the lungs do not collapse after death may be shown in a cat or rabbit. Open the abdominal cavity, and pull back the liver and stomach. The pink lungs may be seen through the thin diaphragm. Pull the diaphragm back to see that the lungs follow it, keeping in contact with it all the time. Now lightly puncture the diaphragm on one side. The lung on this side collapses, but the lung of the other side remains expanded, showing that the two pleural cavities are separate.

The Movements of Respiration. - The process of respiration consists of two acts, inspiration and expiration. In inspiration the principal active forces in the body are, first, the diaphragm; and second, the muscles which elevate the ribs.

The diaphragm is a muscle, and when its fibers shorten, the diaphragm is pulled down. In moving down it presses on the abdominal organs, and makes the abdomen protrude laterally and ventrally. This lowering of the diaphragm increases the space in the chest; the air already in the chest expands to fill this greater space. When expanded it exerts less pressure than before, and the air outside, having greater pressure, enters till equilibrium is produced. The air enters through the trachea, presses on the inside of the elastic lungs, and makes their bases extend, following the diaphragm in its descent. The bases of the lungs remain in contact with the upper surface of the diaphragm all the time.

Certain muscles of the chest wall elevate the ribs and sternum. This act widens the chest; and the air, as before, presses in through the open trachea, and keeps the sides of the lungs in contact with the inner surfaces of the chest walls.

Inspiration requires considerable effort, because the dia- 
phragm in its descent presses upon the elastic organs of the abdomen (stomach, liver, etc.), and these organs, in turn, are pressed against the elastic walls of the abdomen. It is somewhat as if one pressed a pillow down into a rubber bag; the
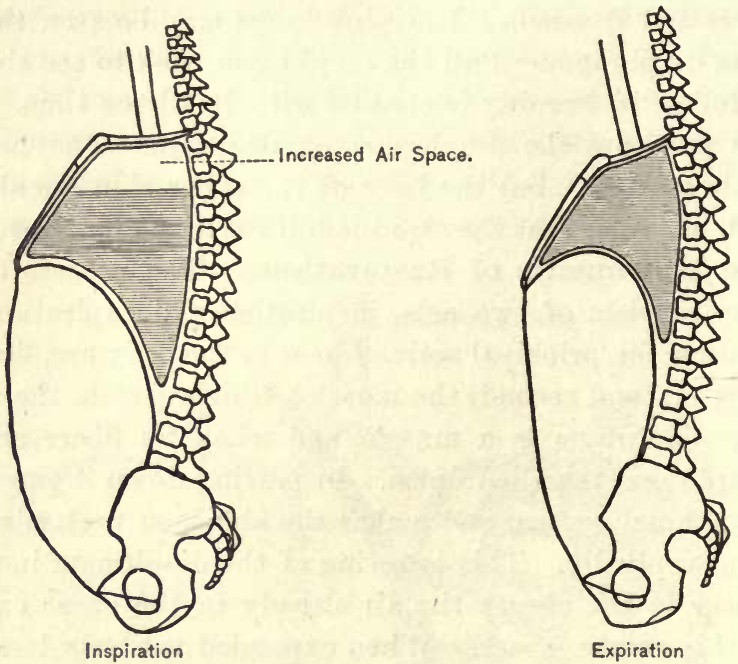

Fig. 45. Diagrammatic Sections of the Body in Inspiration and Expiration.

pillow would spring up as soon as the pressure was stopped, because of its own elasticity as well as that of the bag. Therefore, as soon as the diaphragm relaxes, the elastic walls of the abdomen retreat, and the abdominal organs rise to their former place.

When the ribs are elevated, the cartilages which connect the ventral ends of the bony parts of the ribs with the breastbone are slightly bent. When the muscles relax, the elasticity of the rib cartilages helps to bring the ribs back to their former position, thus reducing the chest to its former width. 
Thus we see why expiration is easy; in fact, "does itself" (in ordinary respiration), by the elastic reactions mentioned. But inspiration is harder than it would be if it were not for the fact that the descent of the diaphragm meets resistance, and the ribs, in rising, have to overcome resistance in bending the costal cartilages, and in raising the weight of the chest walls and shoulders.

When one opens a door that has a spring to shut it, he has to expend more energy to open the door than he would if he did not have to bend (twist or compress) the spring at the same time. But no effort is needed to shut the door. The door was opened and shut at the same time; i.e., when the door was opened, force was stored in the spring (in the form of what is called potential energy), and this stored energy shuts the door while we pass on. We can better afford to employ more energy while opening the door, than to take the extra time to shut it. If, then, a door with such spring were fastened open, it might remain open for a long time. When released it flies shut. If one, in this case, asks, "Who shut the door?" the answer is, "The person who opened it."

So in the act of inspiration we perform a double work in storing energy by which the expiration is performed without active muscular effort.

Reviewing these points we have:-

\section{FORCES OF INSPIRATION.}

1. Depression of the diaphragm.

2. Muscles elevating the ribs.

3. Pressure of the external air.

RESISTANCES TO INSPIRATION.

1. Compression of the abdominal organs, and stretching abdominal walls. 
2. Bending the rib cartilages, and lifting the chest.

3. Stretching the lungs.

\section{ELASTIC REACTIONS OF EXPIRATION.}

1. Elastic reaction of the abdominal walls and contents.

2. Elastic reaction of the rib cartilages.

3. Elastic reaction of the lungs.

Thus far we have been speaking of ordinary respiration. In forced respiration, as in shouting, many muscles are brought into play to expel the air rapidly and forcibly. In such an act as coughing there is vigorous action of the abdominal muscles.

The main part of respiration is performed by the diaphragm; and the more common mode of respiration is therefore called abdominal, or diaphragmatic, respiration. In women of the civilized races, respiration is more largely accomplished by the action of the thoracic muscles, and is called thoracic, or costal, respiration. In children the respiration is of the abdominal type.

Ordinary inspiration takes slightly less time than expiration.

The rate of respiration in the adult varies from sixteen to twenty-four per minute, the average being about seventeen times a minute; about one respiration for every four heartbeats. Light is favorable to respiratory activity.

The rate is affected by the position of the body, state of activity, temperature, digestion, emotions, age, disease, etc.

Coughing is a forcible expiration, usually directed through the mouth, and for the purpose of getting rid of some foreign substance, or caused by irritation. In sneezing there is first a deep inspiration, and then the current of air is forced out, chiefly through the nose. Sneezing may be prevented ky pressing firmly on the upper lip. Crying, laughing, sobbing, 
are modifications of respiration connected with certain emotions. Yawning and sighing are deeper breathings, caused by ennui, depressing emotions, or a deficient ventilation. Hiccuping is sudden inspiration, produced by spasmodic action of the diaphragm, accompanied by sudden closure of the glottis, and is often caused by some disorder of stomach digestion. Snoring is caused by breathing through the mouth, and setting the soft palate into vibration. Sniffing is sudden inspiration: the diaphragm is suddenly pulled down, the air in the nasal cavity is thus drawn downward, and the air we wish to test, or the odor we wish to inhale, is thus drawn into the upper nasal cavities; whereas in ordinary inspiration most of the air passes along the lower part of the nasal passage. In hawking, the air is forced out through the narrowed passage between the root of the tongue and the soft palate to remove mucus. Gargling is forcing air up through liquid held between the tongue and the soft palate.

Capacity of the Lungs. - Have the class stand, and each pupil hold up his right hand.

1. Let all breathe together, at the ordinary rate and depth, and let the hand rise about three inches during inspiration, and fall again during expiration. The amount of air taken in at an ordinary breath is from 20 to 30 cubic inches, or about a pint. This is called the Tidal Air.

2. As before, let the hand go up and down with the breathing, but at the end of the third inspiration, instead of stopping with the usual amount, keep on breathing in as much as possible, letting the hand rise accordingly. This air that can be taken in above the ordinary breath is called the Complemental Air, and it is estimated to be, on the average, about 120 cubic inches.

3. Begin as before, and at what would be the end of the third expiration continue to drive out as much air as possible, 
indicating the degree by correspondingly lowering the hand. This air that can be breathed out beyond the ordinary expira-

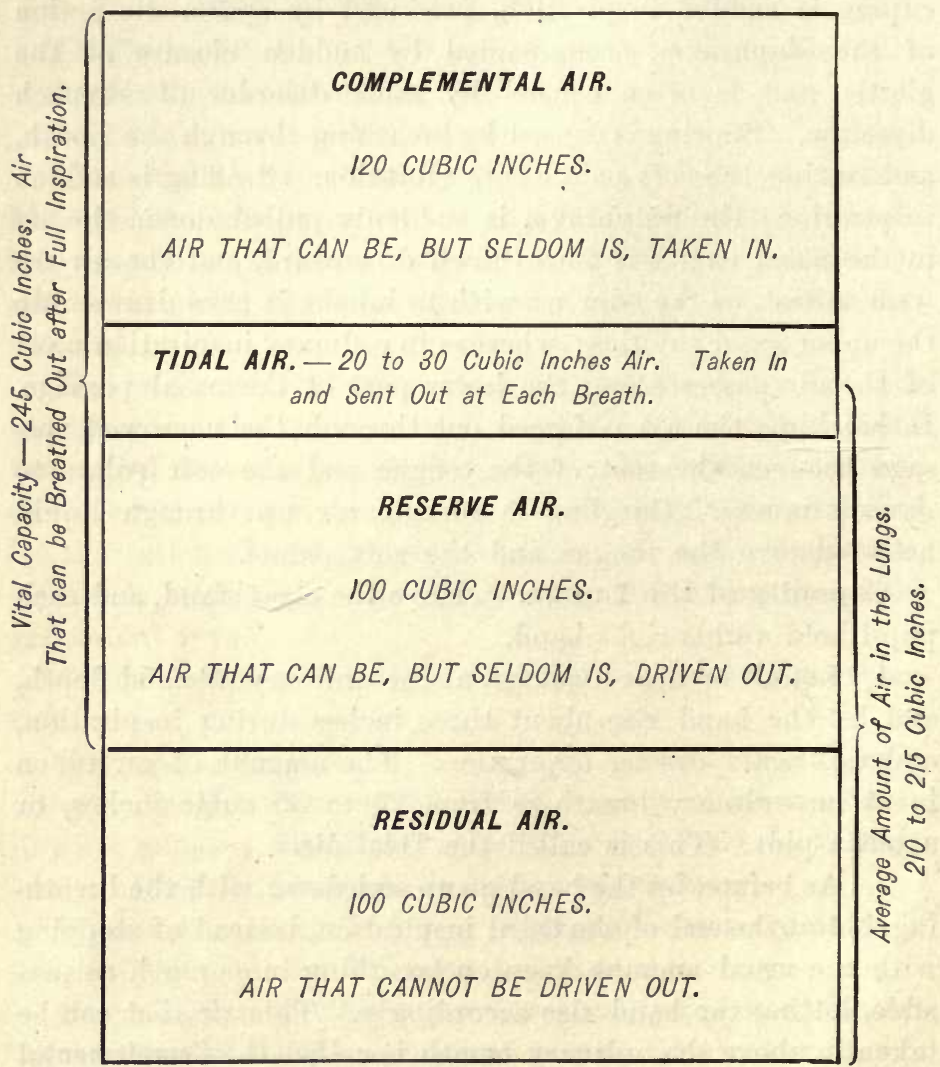

Fig. 46. Diagram of Lung Capacity.

tion is called the Reserve Air, and is reckoned at about 100 cubic inches. 
4. The air cannot all be breathed out. The remainder is called the Residual Air, and is computed to be about 100 cubic inches.

All the air that can be breathed out after a full inspiration, i.e., the sum of the complemental, tidal, and reserve air, would be about 240 to 250 cubic inches, and is called the Vital Capacity.

Of course these figures represent only the average of certain experiments and observations. By practice any one can considerably increase his vital capacity.

A simple method of measuring these stages of respiration is to graduate a gallon bottle carefully to pints by pouring in water and marking on the outside with a file. Then invert the bottle in a trough of water, and inhale from it by means of a rubber tube. Or fill the bottle, invert in water, and exhale into it.

It will be observed, since the same tube that takes in the fresh air also sends out the bad air, that when the current is reversed the first air to be sent out is the last air that came in, which is, therefore, the best air in the lungs. But the amount taken at each inspiration is three or four times as great as the capacity of the trachea and bronchi, so that at each breath two-thirds or more of the fresh air is taken directly into the air vesicles. In addition to this the better air in the trachea is mixing with the poorer air in the air vesicles by diffusion; the oxygen being more abundant in the trachea, passes down into the deeper parts of the lung, while the carbon dioxid, so abundant in the vesicles, passes up into the trachea.

At each inspiration the air in the lungs receives about oneeighth of its volume of fresh air, and at each expiration about one-ninth of the air is sent out. But it must be remembered that this one-ninth sent out is not the worst, but the very best, 
air that is in the lungs, which has lately entered the air tubes, and part of which can only by diffusion reach the air vesicles, where the exchanges are taking place between the air and the blood.

Hygiene of Breathing. - If one had a barrel of water with a lot of mud in the bottom, and something kept stirring up the mud, of course the water would be muddiest near the bottom. Now, suppose a cupful of the water from the top were dipped out and thrown away, and a cup of pure water were poured in. This method of renewing the water would be like the renewal of the air in the lungs, in that the least bad material is removed, while the addition of fresh supply is made at the point where the material is least vitiated. We see that here we might help matters by dipping faster, or by using a larger cup. We cannot well breathe faster, unless we are exercising, but we can take more air at a breath; and this we ought to do as often as we can, not only full inspirations, but deep expirations as well. Frequently during our study hours it will pay us to stand erect and take a number of full breaths, with the window open (if the weather will permit), so that the quality of the air may be as good as possible. The position while sitting ought to receive careful attention, for a cramped position will reduce the quantity of air taken in at each breath. It is a good general rule to sit with the lower part of the back against the lower part of the chair-back. If one forms this habit, and resists the temptation to slide down in the seat, thus curving the body and cramping the lungs, it will be of great benefit. In walking, it is of great value to the lungs to have an erect carriage, as well as greatly improving the personal appearance. To hold the chest well forward, and the chin well in, is of the utmost importance to every one, and should not be left to the military cadets. It will aid the health and appearance of other students as much 
as it does in the case of these young men, whose health and carriage are universally admired.

Those persons who take constant exercise in the open air are not likely to suffer much from deficient respiration. But persons following sedentary occupations, such as that of the student, not calling for deep breathing (and often the air taken in is of poor quality), need to pay especial attention to the matter.

The fact has been noted that breathing directly aids the circulation of the blood. This is due to the way air pressure is made to affect the large veins. Breathing also may very considerably aid the flow of lymph. Every deep inspiration brings pressure to bear on the main lymph duct as the diaphragm descends. Every forced expiration has the same effect. Now, we must keep in mind that the tissues are fed directly by the lymph that surrounds them; that while the lymph is continually fed by the blood, there is not a great pressure given in this way. The lymph stream is largely dependent on the pressure of the surrounding organs. When one takes a good deal of muscular exercise the lymph is renewed with rapidity enough to supply the tissues with food, and to carry away their wastes. But for those who sit quiet a large share of the day, taking no more exercise than is necessary to take them to and from their places of business, it is well to give this matter especial attention. Deep breathing, regularly practiced several times a day for a few minutes at a time, is very beneficial.

It is a grateful relief to the whole system to stand, stretch, inhale deeply and slowly several times, and to repeat this every hour or so. Every one engaged in office work or studying should form this habit, especially if he does not give an hour daily to exercise in a gymnasium, or otherwise.

Respiratory Sounds. - During respiration sounds are 
produced by which the skilled physician can tell much as to the condition of the respiratory organs.

The Control of Respiration. - Breathing is an involuntary act. Still we can modify it. We can hold the breath for a time; but it is stated that one cannot hold the breath long enough to produce death by suffocation.

The muscles of respiration are under the control of nerves. The center of respiratory control is believed to be in the lower portion of the spinal bulb. This Respiratory Center is one of the most vital points in the body; for if it is destroyed, breathing is completely stopped, and death ensues. This center is affected by the condition of the blood. For instance, if the blood going to this center has not enough oxygen, the center hastens the process of breathing by nerve impulses sent to the muscles of respiration.

The diaphragm is under the control of the phrenic nerves, which arise from the third, fourth, and fifth cervical nerves. If the neck is broken above the point where these nerves are given off, death almost always immediately follows, because the connection of the respiratory center and the diaphragm is broken.

\section{COMPOSITION OF THE AIR.}

Dry air contains, by volume, about as follows :-

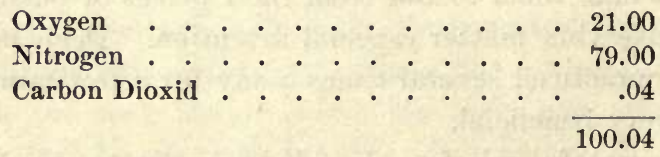

Various gases are found in the air in small quantities. About one per cent of what is classed as nitrogen in the above table is now known to be a distinct element called Argon.

If we would understand the results of respiration, how the air does its proper work in our bodies, and how our bodies 
produce the changes in the air that passes through the lungs, we must know something about the gases that are the constant components of that which we breathe.

\section{EXPERIMENTS ILLUSTRATING THE CHEMISTRY OF RESPIRATION.}

Materials. - Soup-plate or basin, two quart fruit-jars, piece of phosphorus half the size of a pea, chalk crayon, strip of sheet-lead one inch wide and eight inches long, fine wire one foot long, pail of water, eup, matches, watchspring, magnesium ribbon four inches long, two horse-radish bottles, four tumblers (two should be kept in a cool place), lime-water (prepared a day or two beforehand, by putting a piece of quicklime about the size of a hen's egg into a quart of water; pour off the clear liquid for use in experiments), wax tapers, rubber tubing (two feet), bellows, splints, corks, apparatus for generating oxygen and carbon dioxid (consult any chemistry).

Caution. - Always handle phosphorus with forceps, not with the fingers. Cut it under water, in a plate.

Preparation. - Hollow out the large end of the crayon, and wire it to one end of the lead strip. Bend the strip so that the crayon cup will be held at half the height of the jar, and set it on the plate. Lay the piece of phosphorus in the chalk cup made of the crayon; pour a little water into the plate.

EXPERIMENT 1. - Ignite the phosphorus, and lower the inverted jar over it. Keep the mouth of the jar covered with water, adding more if some of the water is drawn up into the jar.

Explanation. - The phosphorus in burning unites with the oxygen of the air, forming a white cloud of phosphoric oxid. This is gradually absorbed by the water, and the pres. 
sure of the outside air pushes water up into the jar to take the place of the oxygen that has been removed. The clear gas left when the cloud disappears is nitrogen. What part of the jar is occupied by the nitrogen? What part of the air is oxygen? Of course this experiment will not show these proportions very accurately.

Experiment 2. - After the gas remaining in the jar has become clear, carefully lift the plate and jar, and lower them into a pail of water until the mouth of the jar is well under the water. Hold the jar with one hand, and let the plate and lead sink. Slip the palm of the hand over the mouth of the jar, and invert the jar and set it on the table, keeping the hand firmly over the mouth of the jar. Slowly lower a lighted taper into the jar. The flame is extinguished. Quickly cover the jar and repeat the experiment. The nitrogen does not support combustion. If an animal were put into a jar of nitrogen, it would soon die; not because the nitrogen injures it in any way, but simply because nitrogen does not support respiration.

Nitrogen makes up four-fifths of the air and oxygen onefifth.

Experiment 3. - Into a jar of oxygen lower a splinter with a live coal at the end (left after blowing out the flame). The coal is kindled into flame. This shows the chief property of oxygen; that is, its power of supporting combustion.

Experiment 4. - Into a jar of carbon dioxid lower a lighted taper. It is at once extinguished.

Carbon dioxid may easily be generated by such means as are at hand in every kitchen. Baking-powder liberates carbon dioxid freely when water is added to it. Put some bakingpowder into a tumbler, and pour water upon it. The carbon dioxid may be poured into another tumbler and tested. Better put the baking-powder into a bottle, or Florence flask, fitted 
with a cork having a tube (glass and rubber), so the gas can be collected. Or the baking-powder may be placed in a saucer, a funnel inverted over it, and a rubber tube slipped on the end of the funnel for a delivery tube. Collect two jars or tumblers of earbon dioxid.

Experiment 5. - Into a jar or tumbler of carbon dioxid pour a little lime-water, and shake it vigorously, holding one hand over the top of the jar. The lime-water is turned milky. This is the test of carbon dioxid.

Experiment 6. - Pour a little lime-water into a jar, and breathe through it by means of a glass tube. The lime-water is rendered milky, thus showing the presence of carbon dioxid in the expired breath.

EXPeriment 7. - Invert a jar over a burning taper. The light is soon extinguished. Pour a little lime-water into the jar, and shake it actively. Is carbon dioxid produced by the burning taper? The oxygen of the air unites with the carbon of the taper and forms carbon dioxid.

Experiment 8. - Over a burning taper invert a clean, cold jar. A film of moisture is seen on the inside of the jar. This water vapor was produced by the burning of the taper.

Experiment 9. - Breathe into a cold glass jar. The moisture from the breath is condensed on the inside of the jar.

Experiment 10. - Burn a watchspring in oxygen. Iron oxid, or iron-rust, is the only product, while much heat and light are given off.

EXPERIMENT 11. - Place a piece of watchspring in a glass of water. The next day we shall find it has rusted. This rust. is iron oxid, the same as the iron oxid produced by the rapid oxidation which we called combustion.

Experiment 12. - Burn a piece of magnesium ribbon. It gives an intense white light, and the only product of the combustion is a white ash, magnesium oxid. 
Experiment 13. - Scrape a piece of magnesium ribbon bright, and place it in a glass of water. In a day or so it will be found to be coated with a white substance, which is magnesium oxid, the same as was produced by the rapid oxidation, or combustion. In the latter experiment we should probably call it magnesium rust.

Experiment 14. - Exhale slowly through a tube extending to the bottom of a fruit-jar. Continue exhaling for a long time, to be sure to drive out the air that was in the jar. Cap the jar tightly, and set it in a warm place for twenty-four hours. It will have a bad odor from the putrefaction of the organic matter thrown out in the wastes from the tissues.

Experiment 15. - With a pair of bellows force the air of the room through a small quantity of lime-water. By continuing the process for a long time it may be shown that there is carbon dioxid in the air, but not nearly so much as in the expired breath.

Experiment 16. - Exhale for a long time through a small quantity of sulphuric acid (chemically pure), using a pipette with a large bulb, so that the acid could not be drawn up into the mouth (the acid is very strong). The acid will gradually grow dark colored, indicating the presence of organic matter.

Experiment 17. - Hold a thermometer at arm's length. It indicates the temperature of the air - of the air that you are breathing in. Breathe for a few minutes upon the bulb of the thermometer, and the fact is clearly shown that the air we breathe out is much warmer than the air that we breathe in.

These experiments show that breathed air has gained : -

1. Heat.

2. Water vapor.

3. Carbon dioxid.

4. Waste products, or impurities, having no definite name, 
because not well known, highly putrescible, often cailed by the general name of "organic waste matter."

Let us compare the inspired and expired air in its composition.

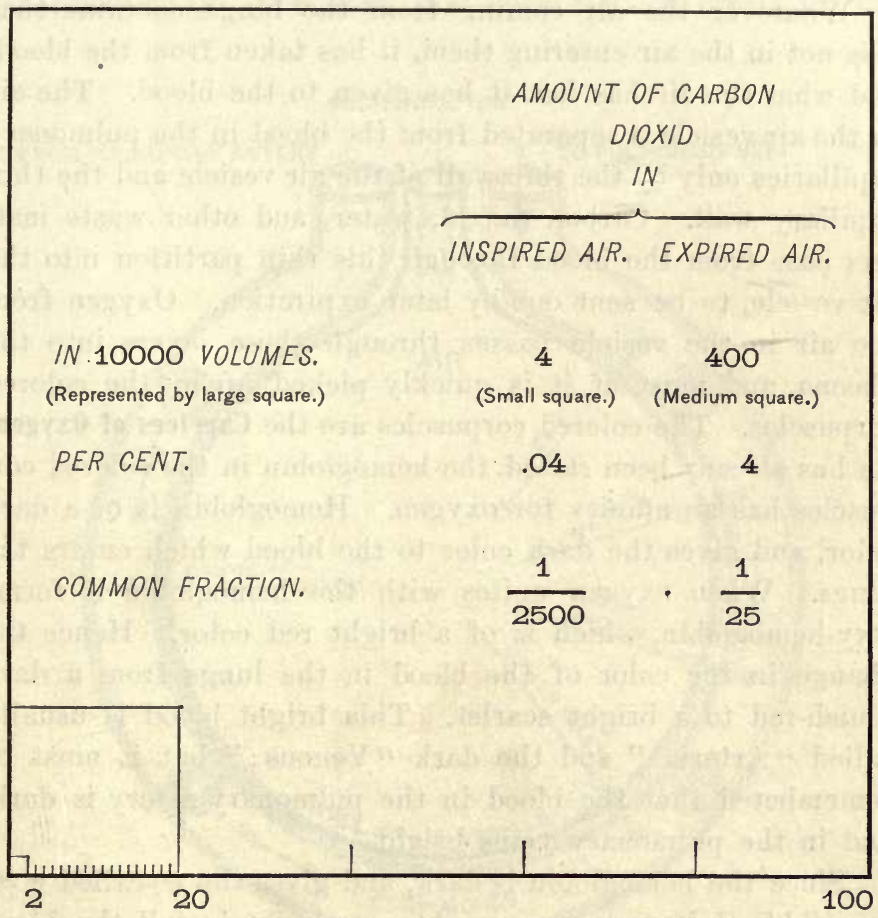

Fig. 47. Amount of Carbon Dioxid in Inspired and Expired Air.

Inspired air contains in one hundred parts, as compared with expired air:

Oxygen. Nitrogen. Carbon Dioxid.
Inspired air . .
21
79
.04
Expired air . . 16
79
4.00 
While the amount of nitrogen remains about the same, some oxygen has disappeared, and its place is taken by carbon dioxid, while the amount of carbon dioxid has increased a hundred-fold.

Whatever the air coming from the lungs contains that was not in the air entering them, it has taken from the blood, and what the air has lost it has given to the blood. The air in the air vesicle is separated from the blood in the pulmonary capillaries only by the thin wall of the air vesicle and the thin capillary wall. Carbon dioxid, water, and other waste matters pass from the blood through this thin partition into the air vesicle, to be sent out by later expiration. Oxygen from the air in the vesicle passes through these layers into the plasma, and most of it is quickly picked up by the colored corpuscles. The colored corpuscles are the Carriers of 0xygen. As has already been stated, the hemoglobin in the colored corpuscles has an affinity for oxygen. Hemoglobin is of a dark color, and gives the dark color to the blood which enters the lungs. When oxygen unites with the hemoglobin it forms Oxy-hemoglobin, which is of a bright red color. Hence the change in the color of the blood in the lungs from a dark bluish-red to a bright scarlet. This bright blood is usually called "Arterial," and the dark "Venous;" but it must be remembered that the blood in the pulmonary artery is dark, and in the pulmonary veins bright.

Since the hemoglobin is dark, and gives the so-called "venous" blood its color, we might appropriately call the blood that enters the lungs "hemoglobin blood." Since the hemoglobin takes oxygen, becomes bright red, and gives the blood its bright color as it leaves the lungs, no more suitable name can be given to it than "oxy-hemoglobin blood." The only good objection to these terms is their length; but since they are exact, while the commonly used terms are inexact, these, 
or similar ones, should be adopted. We might say "oxygenated blood," and "de-oxygenated blood;" but here, again, is a chance for misunderstanding, for the blood still has considerable oxygen when it enters the lungs (unless of an animal suffocated). We have just seen that in breathing we

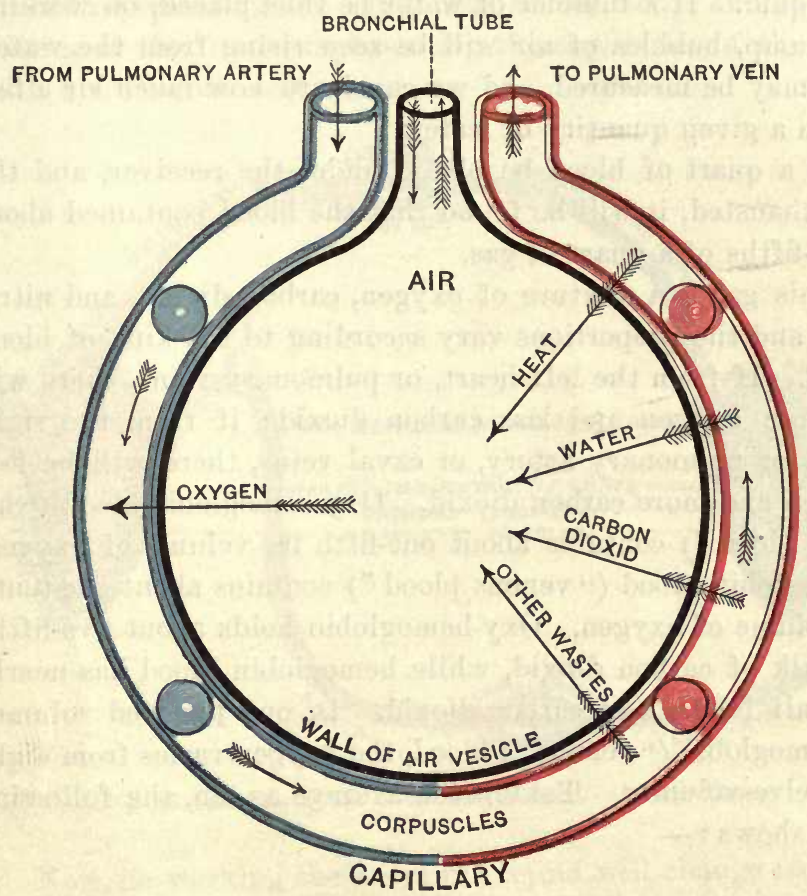

Fig. 48. Exchanges between the Air and the Blood In the Lungs.

only take into the blood about one-fourth of the oxygen of the air that passes through the lungs. In like manner the blood, passing through the tissues, gives up to those tissues 
(in ordinary circumstances) only about half the oxygen it contains (perhaps holding the remainder as a reserve).

How can the amount of oxygen and other gases in the blood be determined? Or how can we find how much air is dissolved in water exposed to air? By placing the liquid under the receiver of an air-pump, and removing the air above the liquid. If a tumbler of water be thus placed, on working the pump, bubbles of air will be seen rising from the water. This may be measured, and we can learn how much air a fish has in a given quantity of water.

If a quart of blood be placed under the receiver, and the air exhausted, it will be found that the blood contained about three-fifths of a quart of gas.

This gas is a mixture of oxygen, carbon dioxid, and nitrogen ; and the proportions vary according to the kind of blood taken. If from the left heart, or pulmonary veins, there will be more oxygen and less carbon dioxid; if from the right heart, or pulmonary artery, or caval veins, there will be less oxygen and more carbon dioxid. Oxy-hemoglobin blood ("arterial blood") contains about one-fifth its volume of oxygen. Hemoglobin blood ("venous blood") contains about one-tenth its volume of oxygen. Oxy-hemoglobin holds about two-fifths its bulk of carbon dioxid, while hemoglobin blood has nearly one-half its bulk of carbon dioxid. In one hundred volumes of hemoglobin ("venous") blood, the oxygen varies from eight to twelve volumes. Taking the average as ten, the following table shows :-

THE GASES IN THE BLOOD.

From 100 volumes of -

May be obtained

Oxygen. Carbon dioxid. Nitrogen.

Oxy-hemoglobin (arterial) blood 20 vols. Hemoglobin (venous) blood . . 10 "

40 vols.

46 "
1 to 2 vols.

1 to 2 " 
The changes that take place in the color of the blood, both in the lungs and in the tissues of the other parts of the body, may be illustrated as follows: Prepare a heart as directed on page 64. Use for the liquid a strong solution of litmus, neutralized or slightly alkaline; place in the throat of each funnel a small sponge. Saturate with ammonia the sponge in the funnel representing the capillaries of the body, and saturate the one in the funnel representing the capillaries of the lungs with hydrochloric acid.

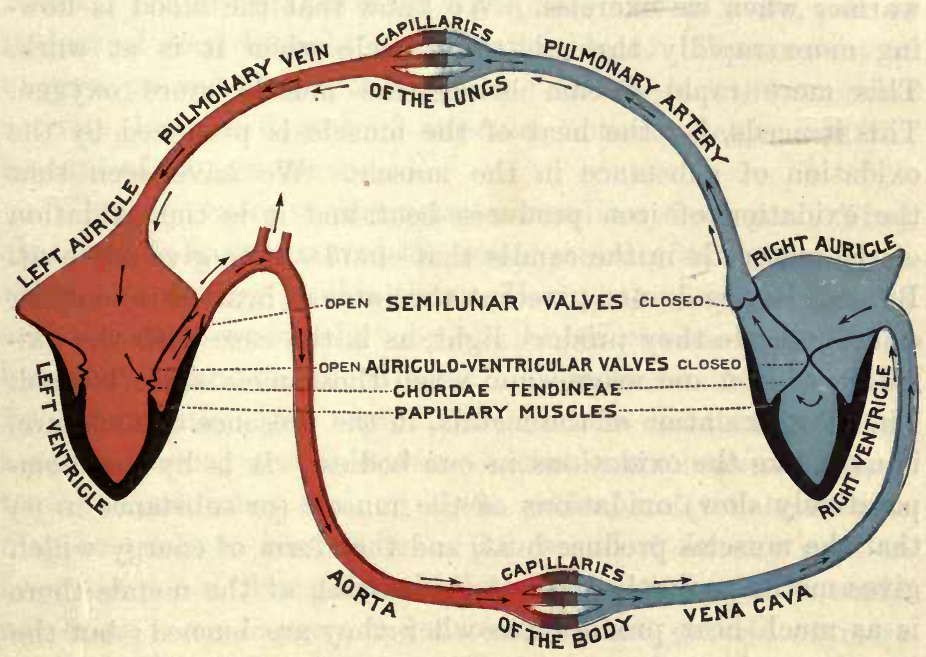

Fig. 49. Diagram of the Circulation of Blood. (Dorsal View.)

Now, on working the heart the liquid will change from red to blue in the funnel representing the body, and from blue to red in the funnel representing the lungs.

"Anatomically there are two lungs, and the heart lies between them; physiologically, the lungs form a single organ, which is interposed between the two hearts." - WILDER. 
What does the blood do with the oxygen that it gets in the lungs? and where did it get the carbon dioxid and other impurities that it brings to the lungs? Let us follow the blood and see. From the pulmonary veins the blood goes to the left heart, and is pumped to all the tissues except the lungs. Let us follow a branch of the aorta that leads to a muscle.

The Production of Heat and Motion in the Body.When a muscle works it becomes warmer. This has been repeatedly proved by experiment. We know that we feel warmer when we exercise. We know that the blood is flowing more rapidly through the muscle when it is at work. This more rapid stream brings the muscle more oxygen. This it needs, for the heat of the muscle is produced by the oxidation of substance in the muscle. We have seen that the oxidation of iron produces heat, and it is the oxidation of the materials in the candle that enables it to give out heat. But our bodies do not give out the intense heat of a burning candle, nor do they produce light, as is the case with the oxidation of iron and magnesium when those metals are burned. The slow oxidation of the metals, in the presence of moisture, is more like the oxidations in our bodies. It is by the (comparatively slow) oxidations of the muscle (or substance in it) that the muscles produce heat, and that form of energy which gives motion. In the case of the rusting of the metals there is as much heat produced as when the $y$ are burned; but the heat is so slowly generated that it is given off about as fast as it is produced, and we do not notice it. The oxidation produces the waste matters, just as the burning of the various substances produces waste.

In our experiments with oxygen we see that substances which burn in air will burn still more actively in oxygen. But we must not infer from this that in our bodies the oxidation of the tissues would be faster in pure oxygen. This is 
not the case. The tissues take as much oxygen as they need (if they can get it); and they will not take any more than they need, no matter how much is offered them. It does not injure the body, nor any part of it, to breathe pure oxygen. It does not make one feverish, it does not produce any more heat, nor make one "live faster." This point should be specially noticed, as it was formerly supposed that the oxidation of the tissues of the body was just like any combustion of dead material. But the tissues are alive. They know their own needs. Each cell takes what it requires and no more, just as it does of food brought to it by the blood. The amount of oxygen present does not determine the degree of muscular activity, but the degree of muscular activity determines the amount of oxygen consumed.

When we exercise, the muscles need more oxygen. They also need to have removed the waste matters that they are so rapidly producing at this time. How is the oxygen brought and the waste removed? By the blood, you answer. True; but what makes the blood come and go faster at this time? By reflex action, you reply. The muscles send a message to a nerve center; and this nerve center sends back a message to the blood tubes, making them widen, and the heart also may be made to beat faster. But would it do any good to have the blood flow through the muscles faster, if it could not bring more oxygen, and take away and get rid of more wastes? You will say no. To give the extra oxygen, and take out the carbon dioxid, the lungs cannot, of themselves, take in and send out air. The work of pumping air depends on the muscles of respiration, the diaphragm, and the muscles that elevate the ribs. These will not work faster unless they are ordered to do so. A message must be sent to these telling of the need in the muscles that we are considering, say one of the large muscles of the lower limbs. Thus, by a 
series of reflex actions, all these processes are kept in harmonious relation to each other. It must be borne in mind that increased blood-flow is the consequence, and not the cause, of the increased activity of the tissues.

The tissues of the body are oxidizing all the time. But when they are in vigorous action they oxidize very much more rapidly.

Insert the bulb of a thermometer into the mouth, and keep it there three or four minutes to find the temperature of the inside of the body. For this it is better to use a clinical thermometer, if one can be obtained. The average temperature of the tissues within the body is about $98.5^{\circ} \mathrm{F}$.

The body may be compared to a stove. Into one we put fuel and produce heat. In the other we get heat from food. But the body is not like the stove in burning the fuel (food) directly. The food is first made into tissues, or "storage compounds" in the tissues. It is as though we were to build a stove entirely of coal, and then start a fire in it. In that case it would produce heat not merely by burning in one place within, but would be burning throughout the whole of its substance. This is the case with the body.

We have seen that the muscles constitute nearly half of the weight of the body. We know, too, that muscular tissue is more active than most of the tissues. We would now naturally infer, as indeed is the fact, that it is the chief source of the heat produced in our bodies.

Next to the muscles, in importance as a heat producer, is the liver, which is the largest gland in the body, and, as we shall soon see, one of the most active. The blood, as it leaves the liver by the Hepatic veins, is hotter than anywhere else in the body.

But it will be better to compare the body to a locomotive, as we produce not only heat, but motion as well. 
If a visitor from another planet, unfamiliar with such creatures as we are, were to observe closely a man and a locomotive, he would see several points in common:-

1. Both are warm.

2. Both move.

3. Both use fuel (food and coal).

4. Both take in air, and (if it were a winter day)

5. Both give off smoke (which is essentially the same in the two, carbon dioxid and water vapor being the chief constituents).

By a closer examination he would find out some of the differences that we have noticed:-

1. That the body does not get hot enough to burn; i.e., the oxidation is relatively slow, and is not combustion.

2. That the oxidation of the body never produces light.

3. That the oxidation here is always in the presence of moisture.

When the breath is held for some time, the carbon dioxid in the expired air may reach 7 or 8 per cent.

During violent exercise the amount of carbon dioxid given off may be from two to two and a half times as much as when we are at rest.

The amount of carbon dioxid given off is increased in cold weather, and by taking food; is from one-fifth to one-fourth less during sleep.

Oxygen is carried chiefly in the corpuscles, but the carbon dioxid is carried in both plasma and corpuscles.

The activity of the tissues from their oxidation does not. necessarily mean that the oxidation is direct; that is, that the oxygen is used as soon as it is brought to the tissue. For instance, in the muscles it is believed that the oxygen is stored in some form, probably in combination, so that it can be used when needed, perhaps much more rapidly than could be sup- 
plied by the respiration at the time. If we study the chemistry of explosion, we learn that it is a very rapid combustion. In the explosives are materials that unite instantaneously, instead of slowly burning, as in the case of ordinary combustibles.

Now, many physiologists hold that a sort of explosive compound is formed in the muscles, and that when the muscle acts it does so as the result of the explosion, so to speak, of this material. And, to carry out the figure, the nerve is compared to the match that ignites the explosive. A little heat is enough to cause the most violent explosion. So the force that passes along a nerve fiber is slight. But it rouses a great amount of energy that lay dormant in the muscle. It would seem to have "touched off" a lot of explosive material that was already there, rather than merely started an action that depends on the comparatively slow process of respiration at the time. We cannot follow this theory farther, as it takes us too deep into the study of chemistry in its most difficult branch, - physiological chemistry.

We may thus sum up respiration:-

The tissues need oxygen; air is pumped into the lungs; this air gives oxygen to the blood; the blood carries it to the tissues.

In oxidizing, the tissues produce energy (heat and motion), and give off waste matter (water, carbon dioxid, etc.); these the blood carries to the lungs, the lungs give them to the air, and the air carries them out of the body.

The pumping of the air in and out may be called mechanical respiration. The changes between the air and the blood in the lungs we will call the ventilation of the blood, and the interaction of the blood and the tissues the real, or internal respiration. (Compare Figs. 38, 48, and 49.)

The Two Breaths. - "Every time you breathe you 
breathe two different breaths; you take in one, you give out another. The composition of these two breaths is different. Their effects are different. The breath which has been breathed out must not be breathed in again."-KINGSLEY.

The air in the vesicles receives from the blood carbon dioxid, water vapor, and other impurities above mentioned. It has been believed for a number of years that the organic impurities constitute the most dangerous element in expired air. Carbon dioxid, though to some extent a poison, is not very injurious in such quantities as ordinarily exist in the air, even in poorly ventilated rooms; while the headache and drowsiness that one experiences in a close room where there are a number of people is due to the reabsorption of these organic matters. It is not due to lack of oxygen, for the oxygen may be reduced to 13 per cent without causing discomfort. A person may breathe air containing one per cent of carbon dioxid, with a corresponding reduction of oxygen, when the carbon dioxid is generated by ordinary chemical processes (as in a small room with a large kerosene lamp, or a gasoline stove); but air having one per cent of carbon dioxid produced by breathing is highly injurious, because it contains the organic impurities above noted, and the term "crowd-poison" has been employed for this material. Later investigators, however, maintain that there is nothing poisonous in the freshly expired breath.

\section{HEATING AND VENTILATION.}

We often hear the expression "Free as Air." While air is free, and there is an abundance of it, still the expression is practically untrue. In the temperate and colder parts of the world, and for many indoor workers, especially those follow- 
ing a sedentary pursuit, it is necessary to heat the air for a considerable part of the year. When one is actively exercising his muscles he may keep warm outdoors through our winter days. But, as we see, the heat of the body depends on its internal fires, the oxidation of its tissues. If we are inactive, these fires burn feebly, and we need outside heat. While air is free, it really costs a good deal of money to have it properly warmed. We must have a constant renewal of air or we suffer greatly. One serious trouble is that many cannot afford (or think they cannot) to heat the air properly, and many others are ignorant concerning these matters.

Lung diseases are rare in the regions where the windows and doors may be kept open most of the days of the year. It is from shutting ourselves in so closely that we suffer. 'This is especially true where many people are housed in a comparatively small space, as in many public buildings. But in our private dwellings, even when the owners are amply able to secure the most sanatory appliances, defective apparatus is often put in. Any system that does not provide for a constant renewal of the air is defective.

Grates will aid largely in renewing the air. Although in themselves they merely have provision for sending radiant heat out into the room and much air up the chinney, yet, without any special provision for inlet of air to the room, they draw air in through every crack and crevice. It would probably be very much better to have special ducts for the admission of air which is suitably warmed while on its way into the room, and to make the doors shut snugly, and to have double windows, as then both the admission of fresh air and the regulation of heat will be better secured. But it is a serious question whether, with all our modern appliances and conveniences and luxuries, uniform heat, electric and other regulators - whether, on the whole, we have better air in 
our houses, and take cold less frequently, than in the days when we depended more on the fireplace, even if we did "roast on one side while we froze on the other." Fireplaces are expensive as mere heaters, but they are excellent ventilators. If small ventilating flues could be built around the flue of the main heating apparatus, and connected with the various rooms of the house, air could be drawn from these rooms by ascending currents created by the heat of the central smoke flue. Such flues surrounding smoke flues would have the added advantage of protecting the house from fire through the too common "defective flue."

The General Principles of Ventilation. - 1. Diffusion. Gases tend to mix. We know that if a bottle containing an odorous substance be opened in a room where there are no air currents the odor tends to spread equally through the room. So if a person is in one corner of a large room, where there are no inlets or outlets, and no currents, as he uses the oxygen immediately around him the oxygen farther away will diffuse toward him, so that he will continue to get oxygen till the amount of oxygen in the room is nearly exhausted. So, too, the gases that he breathes out will not remain confined to the space directly about him, but will spread nearly evenly throughout the room. The same takes place in the open air, without wind. So, then, if the windows and doors are open, the air of the room will, by diffusion, be renewed.

2. Wind. Motion of the air renews faster than mere diffusion. Strong wind forces its way through the cracks around windows, and when windows are open on opposite sides of a room there is usually enough breeze to renew the air. But during the greater part of the year this cannot be done.

3. Artificial renewal of the air. The renewal of the air in most cases depends on the fact that heated air rises. Heat expands air. It is then lighter, bulk for bulk, than cooler 
air. The heavier surrounding air presses the lighter air upward. If there are outlets above and below, the heavier, colder air will press in at any opening left below, and push the lighter, warmer air out above. 'Thus, in the case of the common stove we very well know that there are currents of heated air rising above the stove. Children make whirligigs and various toys to place in these up-currents above stoves. Air is, at the same time, flowing toward the stove along the floor and lower part of the room. Cold air can usually be detected entering around the windows and doors, which presses downward and toward the source of heat. The stove does not do much to renew the air in the room except in this general way; some heated air escapes at openings. in the upper part of the room, and some is passed out through the stove, taken in as a draft. But in the main, the action of the heat of the stove is to make a current of warm air up from the stove, which current passes along the ceiling to the more distant corners of the room, then descends, joining the cold air, and repeating the round.

In some cases a jacket is placed around a stove, and a duct from the outer air connects with the lower part of the space inside of the jacket and outside of the stove. Then as the air heated by the stove rises, fresh air is drawn in from outside to be warmed. In this case the direct heat from the stove is shut off from the room. Heat radiates in straight lines. When one holds out his hands beside a stove the heat he receives is radiant heat. Most of the heat from a grate is radiant heat. But in a jacketed stove the heating by air currents is called heating by convection. Now a furnace is practically a jacketed stove (almost always placed in a basement). Furnaces have this good feature that they are all the time sending fresh air into a room.

Although in private dwellings heated by furnaces there is 
no special provision for the escape of foul air, there is ordi. narily sufficient renewal of the air. But in public buildings there should be escape flues for foul air.

Frequently in later years a large foul-air shaft is built in some central part of the building, and a small stove placed in it to create a sufficient up-current.

In many public buildings the currents created by heat are insufficient to renew the air properly. Fans are used, which force the air, properly heated, into the room.

In heating by steam or hot water, if the radiators are placed in the room they give direct, or radiant heat. This system is called "Direct Heating." In itself it has no provision for renewing the air. It gives direct heat, and produces air currents within the room; and any change in the air is wholly incidental, from escape of heated air in the upper parts of the room and corresponding suction of outside air through such openings as the carpenters have left below.

In "Indirect" heating, coils of steam or hot-water pipes are placed in air shafts which lead up to the rooms above, and also have ducts to the outside. As the air is heated by the heat of the pipes, it is forced into the rooms above, by fresh, cold air pressing in through the ducts, to be, in turn, heated and sent up. If there is at the same time a proper escape for the foul air, this makes an excellent system. In many situations the direct and indirect may be advantageously combined. Where there is a grate in a room it serves very well as a foulair shaft, especially when there is a fire in the grate. It is well to have the flue from the grate in the same chimney with that from the smoke-pipe, as then the heat from the smoke will cause a constant up-draft in the grate flue, whether there is a fire going in the grate or not.

With a grate, in private houses, there is ordinarily no need of other foul-air shaft for any room. But it is very desirable 
to have at least some indirect heat, so that the fresh air introduced will be sufficiently heated.

If the introduction of air is thus provided for, it is then safe to put on double windows and make the cracks around the door very tight. Without any special provision for the renewal of the air, these cracks are the means of safety.

In houses heated by furnaces, steam, or hot water, the floor is likely to be warmer from the escape of heat from the heater itself, and from pipes or air ducts under the floor.

There is a very common misunderstanding as to the cold felt near a window in cold weather. It seems that air is entering; but a little reflection will show that even if the window were air-tight this effect would be produced, for the air near the window is cooled by losing heat to the outer air. The air next to the window, thus cooled, is heavier, and falls to the floor; and if there is any source of heat in the room, this cold air will pass along the floor to that source of heat, up from the heating body to the ceiling, and across the ceiling, and so on around again. There may thus be currents without any appreciable change in the quality of the air. It is economy to use double windows, and prevent the loss of heat through the glass. So both economy and comfort suggest to us that we reduce as much as possible cracks around doors and windows, use double windows, make vestibules at entrances, and build special ducts by which fresh air may enter, and heat it properly on its way in.

Breathing through the Mouth. - We should breathe through the nose, and not through the mouth. The nasal passages are fitted for the introduction of the air (1) by being narrow, but with an extended area; (2) the lining membrane is richly supplied with blood and (3) secretes an abundant supply of mucus. The air, coining through this narrow channel, is warmed, and a large part of any dust it may contain is caught 
by the sticky mucus that covers all the walls of this passageway. If we breathe through the mouth (especially out-ofdoors in cold weather), the air may not be sufficiently warmed before entering the lungs, and much more dust would be carried into the lungs. Then, too, the air has a drying effect on the throat, whereas the mucus of the nasal passages will moisten the air as it enters. The cilia, which extend from most of the cells lining the respiratory passages, are constantly causing the mucus to slowly flow toward the external opening, so a good share of the dust is gotten rid of. A further advantage of breathing through the nose is that we detect odors, and can thus judge of the quality of the air.

\section{DEAD DUST.}

Every one will recall how delightfully refreshing the air is after a rain or a snowstorm. This is not due merely to the fact that the air is cool. It is clean because it has been washed. The rain and snow absorb a considerable amount of the various impure gases that are in the air. But raindrops and snowflakes bring down with them many particles of dust that were floating in the air. Take some of the snow that has fallen in a town. It looks pure in its almost dazzling whiteness. But melt some of it, and you will usually find a decided tinge darkening the water, showing that as the flakes sifted down through the air they caught myriads of particles of dust. Where soft coal is used to any large extent it is one abundant source of this dust. In summer dust has many sources. The dust that blows into your face, and perhaps into your mouth, may be made of dry soil. Take a dry clod and drop it; it falls quickly to the ground. Pulverize it in your hand before dropping it, and considerable of it floats in the air for some time. Any substance that is easily dried 
and pulverized may form part of the common dust. The dust that you wipe from your eye, or is caught by the mucus of the nasal passages, may, instead of being made of clean soil, be from the excreta of horses, decayed leaves, wood, grass, etc. Indoors we are constantly making dust by wearing out our clothes. Many of the tiny particles that we see floating in the sunbeams are bits of cotton or woolen fibers. Shake any. garment in a beam of light to see how much dust is given off, and how easily. The worn-off particles of our shoes, books, floors, all contribute to the ever-present dust.

Now, this dust (so far as it is mere dead, dry matter, not considering it as a poison) is irritating to the lungs and respiratory passages. There is provision, as we have seen, for catching and getting rid of a good deal of it.

But still much is taken into the lungs. Examination shows that the lungs have many black specks from particles of carbon, etc., that have become lodged, and are of no benefit, to say the least.

\section{LIVE DUST.}

Bad as this dead dust is, the injury from it is slight compared to that from live dust. We know that certain seeds float in the air, carried along by the wind. But these are comparatively heavy, and soon sink to the ground.

We all know pollen. At certain seasons it forms, in the vicinity of cornfields for instance, a considerable part of the dust. This is alive. It will grow if it falls on the right kind of a surface, the stigma of the right plant at the right time. Such dust will not grow in our bodies. We do not furnish a soil in which it can grow. It merely adds to the amount of irritating dust.

We have seen puff-balls give off a cloud of dust when they are crushed. Here, again, this dust is composed of live Spores, 
as we call them, that will grow in suitable places and conditions. So, too, from a patch of mold, when brushed, we often see a little cloud of dust. These are a few-instances of kinds of living dust that simply act on us like so much dead matter.

If we set a tumbler of cider on a table in a warm room, in a few days it ferments. This is due to yeast that has gotten into it. Boil the cider to kill any yeast that is already in it, and cork it securely so that air cannot get at it, and it will not ferment. Dried yeast germs float in the air, settle into this exposed cider, and cause it to ferment. Cider is a good soil for yeast. But there are floating in the air many kinds of spores that may grow in our bodies. We know that many of our contagious diseases are due to the growth in our bodies of some of these spores. Our bodies are a good soil for certain germs. The germs that cause consumption, typhoid-fever, Asiatic cholera, erysipelas, diphtheria, and some forms of bloodpoisoning, are well known. Microscopists know them when they see them as readily as we know peas from beans. And it is proved beyond all doubt that these germs get into our bodies by being breathed in, or by being eaten in food, or in drinking water, or by introduction into the blood in wounds. We have reason to believe that small-pox, yellow-fever, measles, and scarlatina are caused by germs; but these diseases have not been studied so successfully.

How can we avoid or get rid of dusts of these kinds?

To exterminate any plant, we try to keep the seeds from ripening, and to kill all that do ripen. Let us take a case that, while not pleasant to contemplate, is too terribly true to allow of being called an imagined case.

A consumptive expectorates on the pavement. In this sputum are probably hundreds, if not thousands, of germs known as bacilli (Bacillus tuberculosis). They are alive. 
Now, so long as they remain on the pavement they do no harm. The sputum dries. But the bacilli are not killed by drying. With other dry material from the pavement they form part of the common dust. Any one of us may breathe some of this kind of matter any day, for there are persons afflicted with this dreaded disease in every community. Our bodies furnish the very best soil for them to grow in. We do not need to go into the street to be exposed. Who knows what he brings into the house adhering to his clothing? These germs may be brought into the most cleanly houses in these ways, or by the wind.

Now, of course, all such material known to be highly dangerous ought to be destroyed. If those suffering from such diseases were careful to burn all such matter, most of the seeds of this disease would be killed. Thus in time we might stamp out the disease, as a scourge of Canada thistles. But so long as people expectorate upon the floors and pavements, it will be difficult to prevent the spread of such germ diseases.

In hospitals such matters are now looked after with the greatest care, and in private houses where there is intelligence on these subjects. And it is encouraging to note the awakening of the public to the significance of the teachings of modern science on this subject, as shown by the fact that many of the railroad and street-car companies now prohibit spitting on the floors of cars, not merely because it is uncleanly, but on the express ground that it is a means of spreading infectious diseases.

These disease germs are the smallest and simplest of living things. They are plants; and while all of them that are well known have their scientific names, just as the larger plants have, they are all included in one general group designated as Bacteria. 



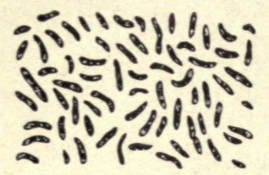

Bacillus of Diphtheria (x 1000).

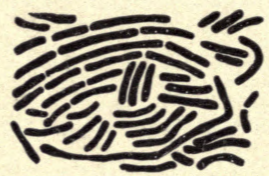

Bacillus of Typhoid Fever (x 1200).

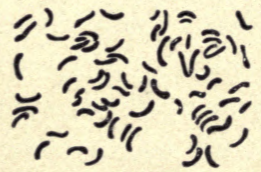

Bacillus (Spirillum) of Asiatic Cholera.

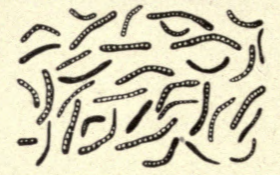

Bacillus of Tuberculosis ( $x$ 1000).

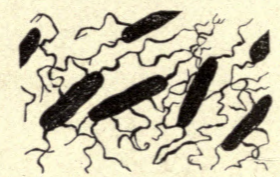

Bacillus of Typhoid Fever (x 1200), showing flagella.

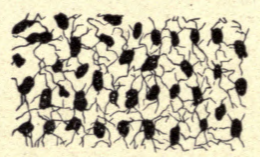

Bacillus of Hog Cholera (x 1000).

TYPES OF BACILLI,

Showing Morphologic Characters and Arrangement.

To face page 147 . 
We need to learn a good deal more about avoiding and destroying dust, and the things that make dust.

Towns and cities need more sprinkling to keep the dust down. Much more of the refuse and street sweepings and cleanings ought to be burned. The dust of a house should always be burned, as we know not what germs of disease may be in it. If we burn it, we shall surely not have to sweep up that dust again. If we send it out-of-doors it may come back, and we may have to handle it again and again.

So far as possible let us avoid things that make dust. When we sweep a carpet, a considerable share of the dust comes from the carpet itself, especially if the carpet is old. Curtains and tapestries of nearly all sorts not only hold dust, but contribute a good deal to it. Those who write on such subjects recommend hard-wood floors with rugs instead of carpets. The rugs can be taken out-of-doors and shaken, and the floors wiped with a moist cloth, so that little dust is left floating in the air of the room. Compare this with the condition that holds after the ordinary sweeping of a carpeted room with the common broom. The dust fills the air, only to settle back on the floor and furniture. Then comes the whisk broom, the so-called dusting. Well, it is dusting! It fills the air once more with dust. But do we get rid of it? Wiping off the dust with a moist cloth takes most of it away on the cloth. For those who cannot have hard-wood floors, a most excellent substitute (and in some respects better) is oilcloth or linoleum. With rugs over these, a house may be kept clean. Not waiting for housecleaning time (that reign of terror), rugs can be taken out, and the floors gone over with a moist cloth.

The improved carpet-sweepers are not only convenient, but sanatory.

Many a well-meaning person will sweep a carpet in a sickroom with an ordinary broom, when the patient is suffering 
from lung disease, thoughtless of the fact that a little dust in sight, and perhaps on the shoes, is of much less significance than dust in the air we breathe. No one likes dust on the floor, but better a thousand times there than in our lungs.

Statistics seem to show that one-seventh of the deaths among the civilized races are due to lung diseases. 'The best authorities are now agreed that consumption is not hereditary. But it appears that there may be inherited a tendency to this disease, so that, if exposed, such persons are more likely to contract the disease than those not so predisposed.

Probably anything that lowers the general vitality makes the system more ready to succumb to any of these contagious diseases. We have all noticed what a difference there is among individuals in the readiness with which they "catch" contagious diseases.

It is believed by some physiologists that the colorless blood corpuscles may take these germs of disease into their substance, and destroy or change them so that the disease is warded off. In other words, they may be compared to a cat that catches and eats the mice which invade a house.

A good general condition of the body helps greatly to ward off diseases of this nature. A cheerful condition of mind and body should be cultivated. In times of widespread contagious disease, if one is terrified into the belief that he is going to have the disease, he is more likely to get it.

'Thorough cleanliness, plenty of direct sunshine, care in diet, and the keeping of the body in good tone, all these reduce the chances of "taking" contagious diseases.

An open-air life, abundant nutritious food, freedom from anxiety, are probably the best restoratives for incipient consumption. The Wilderness Cure is a very interesting book, by Marc Cook (published by Harpers).

Besides the disease-producing bacteria, there are others 
that cause decay and putrefaction of various kinds. They cause our richer foods to "spoil," milk to turn sour, butter to become rancid, etc.

While these bacteria do not cause disease in the human body, they often make food poisonous. The cases frequently reported of poisoning from eating ice-cream, cheese, sausage, etc., are in many cases due to bacteria in them. We should, in the first place, be careful to get good, fresh foodstuffs. In the second place, it should be so kept as to prevent the introduction and development of bacteria in them. Bacteria need heat for their growth (as we so well know is the case with the higher plants). They also need moisture.

So our principal modes of keeping foods from spoiling are to keep them in a cold place, or to dry them. Or we heat them, and then shut them away from the air, as in our various modes of canning and preserving foods. Salting and smoking meats, etc., preserve them by preventing the growth of bacteria. Cold does not usually kill bacteria. So, milk that has been kept in a refrigerator, and that seems sweet, may have in it a stock of bacteria that develop after we drink the milk, from the heat of our bodies. There are now known ways of killing the bacteria in milk and other liquids, known as "sterilizing," that make us safe from this danger.

When we awaken on a cold winter morning, we are likely to find that the fire in our hard-coal stove has burned low. Not enough heat is given out. What is the trouble? Is it merely that more coal is needed? We put another hod of coal in the magazine (though some usually remains). Does this bring the desired result? No. We open the draft. Is this sufficient? It is not. We must shake down the grate and clean out the clinkers. The removal of waste is often more necessary than the addition of a fresh supply of material. It is often a more serious matter to have the waste pipe 
leading to the sewer clogged, than to have the water supply cut off. It is often more to be desired that the garbage cart take away decaying matter, than that the bread wagon arrive. The demands of nature for the expulsion of excreta are imperative, while we can withstand the cravings of hunger for a while. So we shall turn our attention for the present to the immediate demand for the removal of wastes, and later consider the equally important, but less importunate, question of supply and renewal.

Reading. - (1) Bacteria, (2) Dust and Its Dangers, (3) Drinking Water and Ice Supplies, Prudden; Ventilation and Warming of School Buildings, Morrison; Sanitary Conditions of School-Houses, Lincoln (American Public Health Association); Disinfection and Individual Prophylaxis against Infectious Diseases, Sternberg (American Public Health Association); Micro-organisms and Disease, Klein. 


\section{CHAPTER VI.}

\section{EXCRETION.}

\section{THE SKIN AND ITS FUNCTIONS.}

WE have seen that the energies of the body - heat and motion - are produced by the oxidation in its tissues. During this oxidation waste products are formed, which if retained in the body would cause very injurious effects.

How does the body get rid of these substances? We have already learned that the lungs throw off carbon dioxid, water, and certain putroscible organic matter.

The skin is constantly throwing off wastes, collectively called Sweat, or perspiration. Let us first study the structure of the skin.

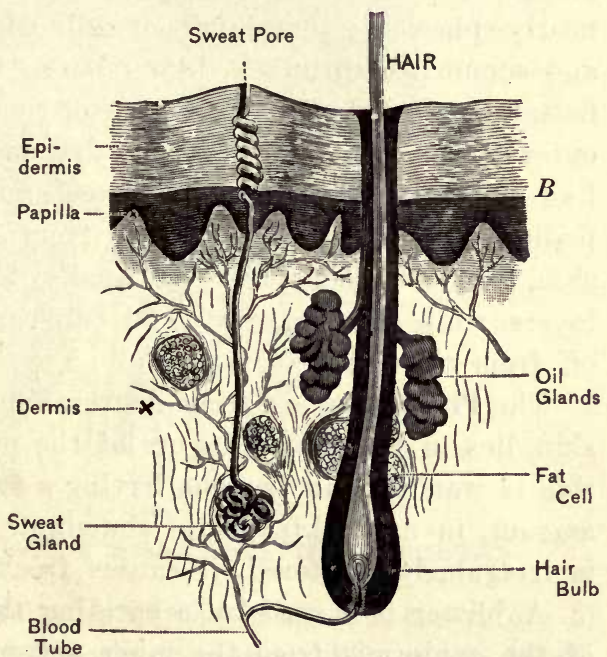

Fig. 50. Vertical Section of the Skin.

The Structure of the Skin. - The skin has two layers, the inner, or Dermis, and the outer, or Epidermis. A bruise 
often loosens or breaks off a piece of the epidermis, but seldom removes the dermis. The epidermis is thick over the palms of the hands and soles of the feet; elsewhere it is thin. Not often seeing the whole thickness of the skin, we do not easily get an idea of its real thickness. The skin constitutes about one-fifteenth of the body's weight, and if tanned, makes a moderately firm and thick leather very much resembling the pigskin used for covering footballs, striking-bags, etc.

The epidermis consists of many layers of cells packed closely together. The deepest cells may be compared to grapes with their cell walls plumply filled out by the liquids of the cell. Suppose, for the inner layer, grapes set on end, and so closely packed together as to press each other into elongated prisms. Then layers less closely pressed, more nearly spherical ; then layers of cells with less liquid in them, and somewhat shrunken, like raisins; then still dryer cells, flattened parallel with the surface of the skin; and last, in the outer part, layers of cell walls, dry and empty, pressed flat like empty grapeskins. The flat cell walls come off in flakes (called dandruff from the scalp) from all the surface of the skin, and new cells are continually formed in the deeper layers, while the old, dry, dead cells are continually thrown off from the outer surface.

The Pigment, or coloring matter, which gives color to the skin, lies in the deeper layers of the epidermis. In albinos this is wanting; in persons having a fair skin it is small in amount, in dark skins more abundant. Where the pigment is irregularly scattered, it causes freckles, etc.

A blister is cansed by separating the outer, harder layer of the epidermis from the inner, softer, darker layer of the epidermis, as shown at $B$ in Fig. 50. Serum, or blood, fills the space between the separated layers.

The dermis consists chiefly of tough fibers, interlacing in 
all directions, somewhat like felt, hence the strength and durability of leather, which is the dermis preserved and prepared in various ways. The epidermis is usually removed in tanning.

The outer surface of the dermis has numerous conical elevations, Papillæ, which fit into corresponding depressions in

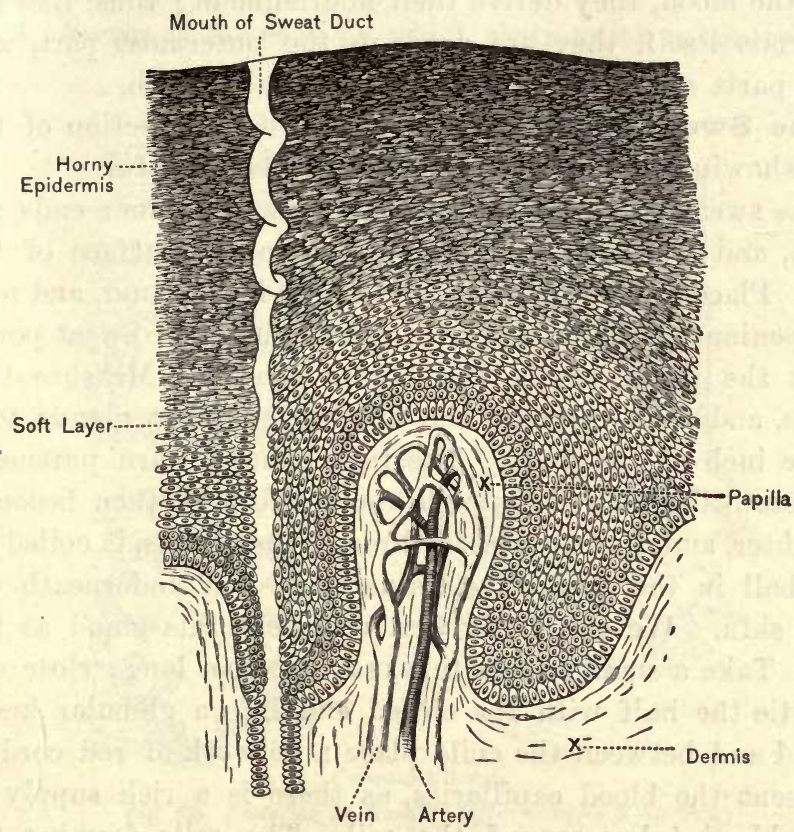

Fig. 51. Section of Epidermis, Showing Papilla. (Highly Magnified.)

the epidermis. In the palm of the hand, etc., the ridges of the epidermis correspond to the rows of papillæ of the dermis underneath. But over most of the dermis the papillæ are irregularly placed, and there are no corresponding elevations of the epidermis. 
The dermis is richly supplied with blood capillaries and with lymph capillaries, but the epidermis has neither. Each papilla is abundantly supplied with blood, having its own network of capillaries.

Hairs and nails are outgrowths of the epidermis. Their . deeper parts are embedded in the dermis, through which, from the blood, they derive their nourishment; thus, like the epidermis itself, they are dead in the outermost part, and these parts are supplied by growth from beneath.

The Sweat Glands. - A model of a cross-section of the skin, showing the glands, hairs, etc., is very helpful.

The sweat glands are minute tubes whose inner ends are closed, and whose outer ends open upon the surface of the skin. Place a linen tester on the palm of the hand, and note the openings of the ducts of the sweat glands, or sweat pores. Count the pores within the square shown. Measure this square, and then estimate the number of sweat glands to a square inch of the palm. The tube going inward pursues a corkscrew-like course through the epidermis, then becomes straighter, and, having passed through the dermis, is coiled up in a ball in the connective tissue lying just underneath the inner skin. We can fairly well represent this gland as follows: Take a small rubber tube, say one foot long; close one end; tie the half with the closed end into a globular knot; around and between the coils place a network of red cord to represent the blood capillaries, as there is a rich supply of these blood tubes around the coil. The cells forming the walls of the coiled part differ from those of the Duct, or straighter part of the tube. As the blood flows around the coil, it gives off lymph; and from the lymph the cells of the gland take certain waste matters, which are passed out to the surface of the skin. There is also some muscular tissue around the walls of the gland. 
The sweat tube is the first example we have had of a Gland. The essential features of a gland are:-

1. Cells lining a cavity, the cells having the power of taking something from the blood (or lymph).

2. Blood supply or lymph supply.

3. A tube or Duct to pour out on some surface the liquid taken from the lymph.

4. Nerves to the cells by which their action is controlled.

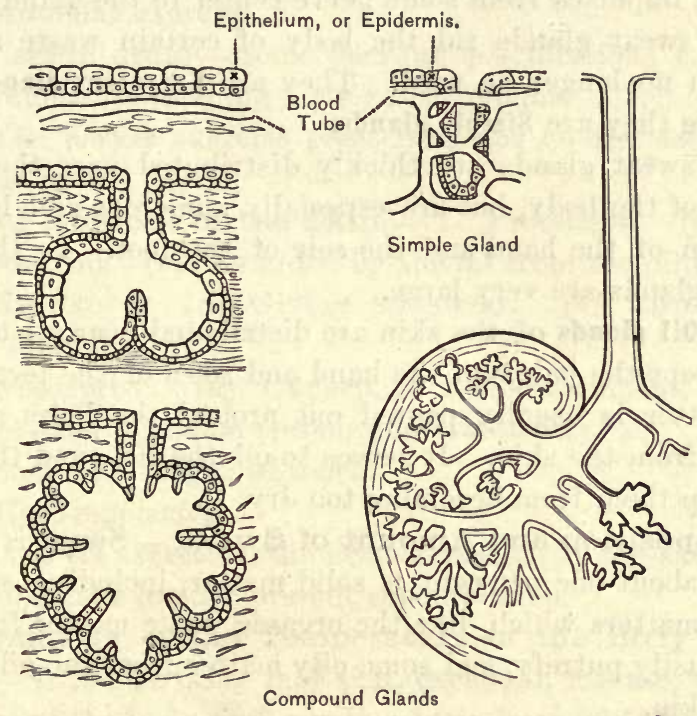

Fig. 52. Evolution of Glands. (After Landois \& Stirling.)

5. (Probably) Special nerve centers controlling the various glands. The cells of the glands in many cases so alter the substances taken from the blood that what is produced by the gland differs from anything found in the blood. The gland may be said to manufacture the liquid it gives off.

The sweat glands, like all glands, are largely dependent on 
the amount of blood supply. In exercising, the skin is usually redder from the greater blood supply, and at the same time the glands are nore active, as they should be; for, during exercise, and immediately after it, there is more waste matter to be thrown out. But the activity of the gland is not a mere filtering process, due to the greater blood pressure. There may be a cold sweat; i.e., when the skin is pale. Here is evidence that the activity of the glands is primarily due to nerve impulses from some nerve center to the gland cells.

The sweat glands rid the body of certain waste matters that can no longer be used. They are Excretory glands. In structure they are Simple glands.

The sweat glands are thickly distributed over the whole surface of the body, but are especially numerous and large on the palm of the hand and the sole of the foot. In the armpit the glands are very large.

The 0il glands of the skin are distributed over all the surface except the palms of the hand and soles of the feet. The oily matter is usually poured out around the hairs as they emerge from the skin. It serves to oil the hair and the skin, and keep them from becoming too dry.

Composition and Amount of Sweat. - Sweat is mostly water; about one per cent is solid matter, including salt and certain matters which, like the organic waste matter from the lungs, easily putrefy, and some oily matter from the oil glands of the skin.

Thrust the hand into a glass jar, preferably a jar that has been in a cool place. Note the moisture that soon gathers on the inside of the jar from the insensible sweat of the hand. A common fruit-jar will do for a small hand; but a candy-jar is better, having a larger mouth and clear glass.

Ordinarily the sweat is evaporated as fast as it is poured out; in distinction from this insensible perspiration it is 
called sensible perspiration when it accumulates enough to be perceptible.

The amount of perspiration is about one quart in twentyfour hours.

The amount varies with : -

1. Temperature, dryness, and rate of renewal of air.

2. Condition of the blood; e.g., if watery from drinking much water.

3. Muscular exercise.

4. Certain drugs - some exciting perspiration; e.g., camphor: others diminishing it; e.g., belladonna.

5. The nerves exercise great influence on the activity of the cells of the gland.

The Functions of the Skin. - 1. Protective. (a) As a tough covering. (b) As aided by the oil from the oil glands.

2. Excretory. (a) Giving off sweat. (b) Also a little carbon dioxid.

3. Absorptive. (a) Oxygen, to a slight amount, may be taken, $(b)$ and medicine (rubbing in ointments).

4. Sensory - organ of touch.

5. Heat-regulating.

Next to its excretion, the heat-regulation by the skin is the most important for our present consideration.

Regulation of the Temperature of the Body by the Skin. - It is a striking fact that, except in disease, the temperature of the body varies only a little from $98.5^{\circ} \mathrm{F}$. in summer and winter, during exercise and rest. The rate of heatproduction varies greatly. The rate of giving off heat must therefore vary accordingly. Otherwise the temperature of the body would soon reach the boiling-point (in thirty-six hours); and, as the body is largely composed of water, it wouid be cooked, and part of it disappear as invisible gas. But long before reaching $212^{\circ} \mathrm{F}$. the tissues would be killed. 
In considering the regulation of the body's temperature, we must bear in mind that the body is surrounded by air almost always considerably cooler than itself. The body is, therefore, almost always giving off heat. Our clothes do not warm us: we warm them, and they keep us from warming the air too fast ; i.e., keep us from losing too much heat. Indoor air in winter in the cooler parts of the United States is kept at about $70^{\circ} \mathrm{F}$. by artificial heat. This air does not warm us. We being about $30^{\circ} \mathrm{F}$. warmer, are warming it.

The skin gives off heat by:-

1. Radiation: heat is given off in every direction.

2. Conduction: whatever we touch that is cooler than our bodies is warmed. We warm chairs, beds, clothing, etc.

3. The air in contact with the skin is warmed and rises. Our body's heat is thus carried off by Convection.

4. The sweat leaving the body is warm; i.e., it takes away heat with it.

5. But the Evaporation of the sweat is a much more important factor in heat-regulation. Any liquid, in evaporating, absorbs heat. The cooling effect of alcohol or ether on the skin is due to the fact that heat is taken from the body in converting the liquid into a gas.

Let the teacher, with a medicine-dropper, place a drop of ether or cologne on the back of the hand of each pupil.

When we put cologne (or alcohol or ether) on the hand or face, we notice two facts: (1) It produces a cooling effect. (2) The liquid soon disappears. To prove that it is not merely that the liquid is cool, try the following: Tie a piece of cheesecloth around the bulb of a thermometer; dip the bulb into a dish of alcohol or ether, and note its temperature (if these are not at hand, gasoline serves very well, or even water, though the evaporation is slower); then lift the bulb out of the liquid, and note any change in temperature. The 
evaporation of the liquid takes heat from the bulb, and causes the thermometer to register a lower temperature. We sponge the face and hands of a feverish patient to reduce the amount of heat. We sprinkle the floor in hot weather, and, by the absorption of heat in evaporating the water, cool the air of the room.

When we exercise, we produce more heat; we sweat more; more heat is taken from the body to evaporate this greater amount of sweat. If we are not exercising, and are in cooler air, we sweat less, and less heat is given off.

This should also be observed: When we exercise, more blood is in the skin, and more heat is given off in the other ways mentioned; when we exercise less, the skin, especially in a cool air, becomes paler, - i.e., has less blood in it, and heat is economized.

Of the total amount of heat given off by the body, eightyseven hundredths are given off by the skin; most of the rest is given off by the lungs; a small amount is given off by the urine, feces, etc.

Distribution of Heat in the Body. - If more heat is produced in one part of the body than in the others, the circulation of the blood tends to equalize the temperatures of the different parts. So, too, if one part is cooled, - that is, is losing heat faster than the others, - the blood brings heat from other organs to that part. For instance, if one holds his hands in the snow, or puts a piece of ice on his wrist, the whole bloodstream is cooled. So if the hands and the feet are exposed to the cold, it may do little good to have the rest of the body covered. A pair of wristers and a pair of leggings or gaiters may often add more to one's comfort than a heavy overcoat.

Regulation of the Production of Heat in the Body. - We have just considered how the temperature of the body may be made uniform by regulating the amount of heat given 
off. But this is not the only fact to be taken into consideration. It seems highly probable that there is a nerve center controlling the amount of heat produced. We know that the heat is produced by oxidations in the tissues. Now, the effect of cold is to increase the amount of oxygen consumed and the amount of carbon dioxid given off, showing that more heat has actually been generated.

We call certain animals "cold-blooded." But the fact that they are less warm than birds and mammals is not so significant as the fact that these cold-blooded animals, instead of maintaining a constant temperature, vary greatly in temperature, being ordinarily only a little warmer than the surrounding air or water. Now, when these creatures are cold, they take less oxygen and give off less carbon dioxid.

Aside from these natural methods of keeping the temperature of the body uniform, we aid the process in more or less artificial ways.

Regulation of Bodily Temperature by Food and Clothing. - When subject to the influence of cold we choose more heat-producing foods, as fatty foodstuffs; we take more vigorous exercise; we put on more clothing, and especially of the non-conducting kinds, - woolens. In warmer weather we eat less fatty matter, wear less clothing, and are less disposed to exercise actively; we fan ourselves to help get rid of heat; we take ices and cold drinks. For most persons it seems better to wear woolen most of the time, as even in summer we are subject to sudden changes in the air, and with such covering one is less likely to take cold.

In getting the clothing wet, the greater loss of heat is not from the coolness of the water, but the loss of heat in evaporating the water from the clothing; and this goes on for a long time. Of course it is very desirable to put on dry clothing as soon as possible; but a person in good health is not very 
likely to take cold, except in very cold weather, if he continues active exercise till he can change the wet garments for dry ones. Children do not often take cold from wading in water so long as they are barefooted; but if they get the shoes and stockings wet, they are likely to take cold, for the reasons above given. Many persons make a mistake in changing their winter underwear for summer too early. In most of the Northern States it is not usually wise to do so until the first of June; but of course no definite rule can be laid down in this matter.

Sunshine. - For good health we need sunshine. Of course we get sunlight indoors, but so do many plants that drag out a sickly existence. We need direct sunlight (when it is not too hot), and many an invalid has been cured by sun-baths. Part of the beneficial effects of sea-bathing is due to sunshine. It is a good thing that it is now the fashion to take a vacation and get well tanned. One of the benefits of the resorts of Colorado (in addition to the climate's mildness) is that there are very few cloudy days during the year.

\section{THE KIDNEYS.}

One important part of the work of the lungs, as we have seen, is to throw out carbon dioxid. The skin also throws off certain wastes. The kidneys have the special task of excreting a waste product of the body called Urea. Urea is the nitrogen-containing waste.

The kidneys are attached to the dorsal wall of the abdominal cavity. The depression of the kidneys corresponding to the stem scar on a bean is called the Hilum. From the hilum issues a white tube, the Ureter, which conveys the urine to the bladder. Entering the kidney alongside the ureter 
is the Renal Artery, a branch of the aorta; and from near the same point the Renal Vein returns the blood from the kidneys, and pours it into the postcaval vein. Through the kidneys is pouring a continuous stream of blood, varying in amount at different times and in different conditions.

The kidney receives a very large amount of blood for its size, as compared with other organs. The flow to it is made easy by the fact that the renal arteries are relatively wide and short, and take the blood directly from the main current of the aorta.

When in active work the kidney is distended with blood. The dead kidney, as we study it, is smaller than during life, just as the dead heart is considerably smaller than when alive.

From the kidney, through the ureter, Urine is continually passing to the bladder. Urine is mostly water, containing urea, salt, and various other substances in small amounts. Urea is a waste matter brought in the blood. If the kidneys are stopped in their action, urea accumulates in the blood, and death soon results; to just the degree that the kidneys fail in performing their duty, just so far must the body suffer. Let us study the structure of the kidney, that we may, in part at least, understand its action.

For dissection, the sheep's kidney, being a little more simple in structure, is preferable; but a pig's kidney is more closely like that of man.

Dissection of the Kidney. - A class can usually be supplied by asking the butchers a week or two beforehand to save them; or, if near a large slaughtering-house, pigs' kidneys can easily be obtained. Butchers do not like to remove the kidneys from the sheep's carcass until it is cut up.

1. Observe the depression in the inner border of the kidney, the Hilum.

2. From the hilum trace the slender white tube, the Ureter, 
back to the bladder. Find also the renal artery and vein, branching as they enter the kidney through the hilum.

3. With a sharp knife split the kidney like a bean, beginning at the outer border, stopping the cut when the cavity, lined by a white membrane, is reached near the hilum. With forceps pry about to explore the cavity bounded by this white membrane. Note the branches of the cavity into the kidney. Note also the extension of the white membrane into these cavities. Make out that the blood vessels extend through these white branches to the outer part of the kidney. Count these branches.

4. In the center of the white membrane find the opening of the ureter, through which the urine is conveyed to the bladder. Pass a probe through this opening into the ureter.

5. Note the difference in color of the outer and inner parts of the kidney. At the line of change of color find where the blood tubes first branch into the real kidney substance. Examine carefully the cut surface of the kidney, to see its markings.

6. Make a drawing of one-half of the kidney as seen from the inside, showing the above points.

7. Cut across the middle of the kidney at right angles to its length, and make a drawing of the cross-section. The projection of the kidney substance into the cavity opposite the ureter is the Urinary Pyramid, and from its apex, through many fine holes, issues the urine which the kidney has secreted from the blood.

Microscopic Structure of the Kidney. - If microscopic sections of the kidney are at hand they should be examined; but the kidney is so complicated in structure that a diagram is needed in connection with the sections and the descriptions. The unit of structure in the kidney is a tube which takes material from adjacent blood capillaries. The relation of the 
capillaries to the tube is peculiar. The inner end of the tube is enlarged into a ball; this ball is deeply depressed opposite the point where the tube leaves it. Into this depression is fitted a globular tuft of capillaries. The arrangement may be illustrated by the common toy known as the "cup and ball." The handle of the cup should be hollow to represent the tube;

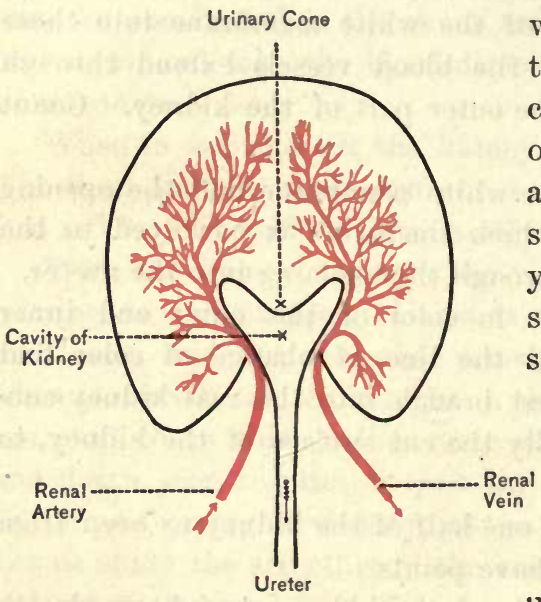

Fig. 53. Cross Section of Kidney. the cup should be doublewalled, the space between the inner and outer layers continuous with the hollow of the handle. Instead of a solid ball held by one string, there should be a yarn ball with two large strings attached to one side, one representing the artery, the other the vein; the yarn ball represents the dense cluster of capillaries. A still better illustration of the urinary tube and capsule may be made thus: Take a thistle tube (used in the chemical laboratory), let down into the bulb a rubber balloon or bag of sheetrubber or cloth, fastening the margin around the rim of the bulb; put a little ball of red yarn in the depression 'of the bag hanging in the bulb; have two ends of the yarn projecting to represent the artery entering and the vein leaving the capsule. The vein, soon after it emerges, breaks up into another set of capillaries which extend around the tube. A number of these primary tubes unite, and many of the common ducts open at the apex of each of the urinary pyramids, 
emptying their secretion into the cavity of the kidney. As the blood flows through the tuft of capillaries in the Capsule at the end of the tube, a good deal of water, together with salt and some other substances, passes through the thin partition into the cavity of the capsule, and thence down the tube. The walls of the tube are thicker than, and its cells are different from, those of the capsule. These cells take the urea and some other substances from the blood, and pass them into the tube to join the more watery material from the capsule.

Comparison of the Skin and the Kidneys. - The kidneys, then, are not very different from the skin. Imagine a pieee of skin rolled up with its outer surface turned inward. Its glands then would pour their secretions into a cavity where they might accumulate instead of evaporating as fast as they are poured out. Of course the kidneys have a somewhat different work from the skin, but in its general plan of working we might say they are skin turned outside in. The kidney unit (the tubular

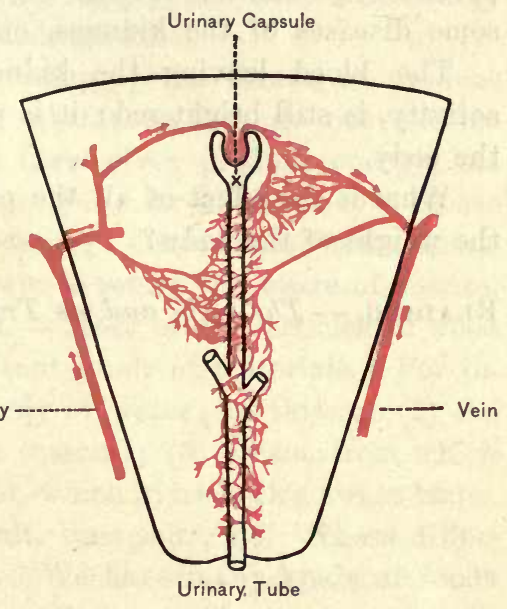

Fig. 54. Urinary Cone, Enlarged. (Diagram.) gland) has branches; i.e., is compound. The kidney is a compound gland of excretion, internal in position. Both skin and kidneys excrete a good deal of water, with salt and some other matter in common. There is a very immediate relation between the work of the kidneys and that of the skin. In 
warm weather, and when exercising actively, we perspire freely, and the amount of urine is reduced; when we exercise less, and especially in cold weather, we perspire less, and the urine is more abundant. Cold drives the blood from the surface. Consequently more blood goes to the kidneys (as well as to the other internal organs), and they throw off much more water, though probably little if any more urea. The average daily amount of urine is about three pints. The quantity is increased by high blood pressure, copious drinking, by cold air (driving the blood from the skin), nitrogenous food, certain drugs, etc. It is diminished by a lowered blood pressure, profuse sweating, diarrhea, non-nitrogenous food, some diseases of the kidneys, etc.

The blood leaving the kidney, especially when in full activity, is still bright red; it is probably the purest blood in the body.

What is the effect of all the processes thus far studied on the weight of the body?

ReAdING. - The Skin and its Troubles, D. Appleton \& Co. 


\section{CHAPTER VII.}

\section{DIGESTION.}

\section{FOOD AND COOKING.}

Thus far we have been studying processes by which the body's weight is reduced. We have studied the oxidation in the tissues and the removal of the wastes. Unless the tissues receive a corresponding supply, the heat and energy of the body cannot long be maintained.

All substances that go to make up the tissues or produce energy are Foods. Certain substances that do not become part of any tissues, nor in themselves produce energy, are useful in aiding the processes going on in the body. These may be called Accessory Foods, e.g., condiments ; some accessory foods, such as coffee, seem to retard the waste of tissues.

Foods and Foodstuffs. - Most of our articles of food consist of two or more different kinds of materials. For instance, milk consists (1) chiefly of water; in this are (2) the substance that makes cheese (casein); (3) cream, from which we get butter (fat); (4) sugar, which gives milk a sweet taste, (5) salts, such as common salt, lime salts, etc. These different materials are Foodstuffs. We have many kinds of foods but few foodstuffs, which we find occurring over and over again, in various forms, in the numerous things we eat.

Foodstuffs are also called "alimentary principles," "nutritive principles," "proximate principles," "food substances," etc.

The foodstuffs are classified into :-

1. Proteids (example, casein).

2. Fats. 
3. Carbohydrates (example, sugar).

4. Water.

5. Salts.

(6. Oxygen is by some authors called a food, but it is more convenient to treat of it elsewhere.)

The Proteids. - The chief substance in the white of an egg is Albumen, a typical proteid. Of the many proteids, some of the more commonly known are Casein (the curd of milk), Gluten (in grains), Legumin (in peas and beans), Fibrin (in blood), Myosin (in muscles). Gelatin (obtained from connective tissue and bones by prolonged boiling) differs considerably from the proteids in composition, but may be counted in with them. It is less valuable as a food than the true proteids, although in certain circumstances more desirable from the fact that it is very easily digested.

The proteids are -

1. Composed of carbon, hydrogen, oxygen, and nitrogen, with a little sulphur, and, in some, traces of phosphorus.

2. Jelly-like, and do not easily diffuse through animal membranes (a characteristic to be kept in mind when studying digestion).

3. Coagulable (usually) by heat, acids, alcohol, etc.

4. Easily putrefy when moist and warm.

The proteids are of special importance as foods because the most active tissues, muscular, nervous, and glandular, and the most important liquids of the body, e.g., blood and lymph, have proteid as a chief constituent. Proteid food, therefore, must be taken to make good the losses of these tissues during their oxidations.

Proteid-Containing Foods. - Lean meat has about twenty per cent of proteid, the rest being chiefly water. Beef and mutton are more easily digested than veal and pork. (For a comparison of fat and lean meat, see Appendix.) 
People who cannot afford to buy tenderloin may take some comfort in knowing that there is really more nourishment in a round steak.

Fish, when fresh, is a good food. Although, as a rule, salted meats are less easily digested than fresh, salted codfish is a nourishing and economical food.

Eggs contain considerable proteid, but their value as food has been overrated. The yolk has a large amount of fat. (See Appendix.) Although the egg has all the material needed to form a chick, it is not a perfect food for man.

Milk, as we have seen, is an ideal food in that it contains all the kinds of foodstuffs, and in the right proportion for the young mammal. But the proportions are not right for the adult. An adult would need four quarts and a half daily, and then he would not get enough carbohydrates (represented in milk by the sugar).

Cheese. - Cheese is very rich in proteid, much more so than lean meat. Yet, as it is rather difficult of digestion we do not use it largely as food; we regard it more as a luxury, while in many parts of Europe it is largely used as food, taking the place of meat. It is a cheap food, and might well be used more extensively, especially by laboring men. It is stated that when taken with milk it is more readily digested.

Vegetable Proteids. - Peas and beans (dried) contain as much proteid (legumin) as meat, and all the cereals contain some proteid (gluten).

Fats. - Fats are composed of carbon, hydrogen, and oxygen. The oxygen is small in amount, so these foods yield a great amount of energy by the oxidation of their carbon (forming carbon dioxid) and hydrogen (forming water). (For further properties of the fats and their importance as food, see Appendix). 
The Carbohydrates and Carbohydrate-Containing Foods. - Sugar and starch are the chief carbohydrates.

Wheat and the grains are chiefly starch (excepting water, which makes up the larger part of nearly all foods), with a small amount of proteid (gluten) and a very little fat.

In ordinary white flour nearly all the gluten has been re. moved with the bran or "middlings." While wheat, or breac made from the whole grain of the wheat, may support life, one would starve if he attempted to live on common white bread alone. It is almost entirely starch. In the "Entire Wheat Flour" it is claimed that all the gluten is retained, only the very thin outer husk of the grain being removed. It does not make so white a flour, but it is better adapted to use as a food. If we use white bread, having thrown away the nitrogenous part of the wheat, we need to take more proteid from other sources than if we used the entire wheat flour. This is not economy. And it is claimed that the entire wheat bread is more wholesome as well as more nutritious. The part thrown away has in it phosphates as well as the nitrogenous material. This flour is ground fine, so that it has not the coarse particles which are in Graham flour, and which are a source of irritation to the mucous coat of the digestive tube in some persons. (See Appendix.)

Water. - Water constitutes about two-thirds of the entire weight of the body. It constitutes the bulk of the liquids we have studied, blood, lymph, sweat, urine, etc. Water is the solvent and carrier of all the material of the body. Hence we need a large amount of it; of course we must remember that we get a good deal of water in most of our solid foods.

Water, as it comes from the clouds, is pure. After enough rain has fallen to wash the air, rain-water is pure, and if caught on a clean roof (especially a slate roof), and kept in a clean 
cistern, it makes good drinking-water. Falling upon the earth the rain-water soaks down until stopped by some impervious layer, such as clay. This water is the supply of our wells and springs. It always has more or less earthy matter in solution, and is therefore called more or less "hard." Unless a good deal of mineral matter or some special material is dissolved in it, it is ordinarily good drinking-water. Such water is not pure, in the strict sense of the word, but is pure for drinking purposes.

The great source of danger is from what are called "organic" impurities. Most bacteria will not live and grow in pure water. They must have something on which to feed and grow. But in water containing a good deal of decaying animal or vegetable matter they are likely to abound. And the most dangerous sources of contamination are cesspools and sewers. Water may be contaminated by such material and not have bacteria in it, but is very likely to harbor such foes. The ordinary cesspool is a grave source of danger. Because a well may be on higher ground than the cesspool does not give assurance that the water may not be polluted. Often when the surface of the ground slopes in one direction, the strata underneath may slope in just the opposite direction, and the well may be the reservoir into which the cesspool is drained.

Good authorities say that a cesspool should not be allowed within a hundred feet of a well.

But it is better and safer to have no cesspool. Where a sewer system is not to be had, it is better to allow no great accumulation of such material. A deep pit in which a quantity of semi-liquid matter gathers is not only a nuisance, but a source of danger. Privies should have a very shallow pit, or none, and should be cleaned often, and the contents disinfected before they are scattered abroad to endanger the lives 
of others. There should be a little dust sprinkled in each day, and occasionally some "chlorid of lime," or sulfate of iron.

Typhoid fever is now known to be usually caused by drinking-water. The dejecta of some one who has had the disease find their way into the source of the drinking-water. In many cases this has been clearly proved. Of course the dejecta of all such patients should be either destroyed or thoroughly disinfected.

Although bacteria will not develop in a cold place, they are not killed in the water when it turns to ice, as was formerly supposed. Further, ice, in forming, does not throw out all the impurities, as was formerly stated. So it is not safe to drink water formed from melted ice unless the water of which that ice was made was good water. The ice taken from ponds is not safe. If ice is made artificially from suitable drinking-water, of course the melted product will be essentially unchanged so far as the composition is concerned. Water may be cooled by placing any ice around it, and we may have the desired temperature without any admixture of a dangerous element.

When one cannot get good drinking-water, or when away from home where the water is of doubtful purity, it is better to boil the water before using it, either as a drink or in preparations of food that are not to be thoroughly cooked. It seems to be proved that it is better to heat the water twice to near the boiling-point than to boil hard once only. The first heating may start the resistant germs into more active life, eausing them to sprout (so to speak), and a second heating several hours later may easily kill them; whereas it has been proved that one hard boiling will not always kill the germs.

Water which has been boiled becomes a better medium than it was before for the growth of bacteria which may afterward get into it, or whose spores may have escaped death in 
the boiling. 'Therefore only enough for one day's use should be boiled at one time: This should be placed in a clean covered vessel, and set in the ice-chest while still warm.

Milk which is to be fed to infants and children should be heated till it steams, thus avoiding danger from tubercle bacilli.

Or if one uses tea and coffee, it is safer to content one's self with these, and not drink much water till that which is safe, as from deep wells, can be obtained.

In hot weather, and especially for those who are engaged in hard work, it has been found that a little oatmeal stirred in the water is beneficial. Do not drink ice-water.

When overheated avoid drinking much cold water. Repeatedly rinse the mouth with cool water, and swallow very little. This is the way trainers manage a horse at a race, and it is sensible to treat man as carefully.

Salts. - Salts include many substances besides common salt. They aid in the solution of various substances during digestion and in other processes. We cannot live without salt.

Lime in the form of calcium phosphate and calcium carbonate is essential, especially in the bones and teeth. Iron is associated with hemoglobin.

Necessity of a Mixed Diet. - Our experience, together with the results of the experiments on animals, teaches that we could not live long if fed on any one class of foodstuffs alone. We must take a representative of each of the groups. We have noticed that most of our foods already contain more than one foodstuff. We so combine them as to get suitable proportions. Thus we eat bread and butter (a small amount of fat with a large quantity of starch and a little gluten), meat and potato, crackers and cheese, pork and beans, egg on toast, bread and milk, rice and fowl, macaroni and cheese; they "go well together," chiefly because they are complementary.

In order to get enough nitrogen from bread alone, one 
would have to eat about four pounds.a day; meanwhile twice as much carbon as is needed would be taken, thus throwing an undue amount of work upon the digestive organs. Again, one would need to consume about six pounds of meat to get the requisite amount of carbon, and six times as much nitrogen as is needed would be taken; to get rid of this extra nitrogen would severely tax the kidneys and liver.

In cold climates a large amount of fat is consumed, while in the tropics starch is the chief food. Our appetites call for more of the fatty foods during the winter season.

While common experience has led people to adopt a mixed diet, the proportions of the different foodstuffs is not always what it should be. The proportions of the foodstuffs (exclusive of water) may be roughly stated as about 1 part of proteid, 1 part of fat, 3 parts of carbohydrates. But this will vary somewhat with the amount of work done, and other varying conditions. (See Tables of Dietaries in Appendix.)

The so-called "vegetarians" recognize the need of proteid food ; and most of them seek proteid in eggs, milk, and cheese. But these are animal products, and the name "vegetarian" is inconsistent. 'They are merely "anti-meat eaters." If they do actually succeed in getting enough proteids from the legumes and the grains, the complete digestion of which is diffcult, they are, as Professor Martin says, to be congratulated on having digestive powers that can stand such a strain. That we are adapted for using flesh as part of our food is indicated in at least two anatomical features: (1) we have canine teeth, though not so fully developed as in the carnivora; (2) the intestine in carnivora is very short, that of the herbivora very long, but in man intermediate.

Tea owes its stimulating effects to a substance called Thein. This is a stimulant to the nervous system, and if not too strong is not followed by a subsequent depression. Tea 
that is too strong is likely to produce nervousness and dyspepsia. Boiling the tea-leaves also brings out the tannic acid that they contain, and produces bad effects.

Coffee owes its stimulating effect to a substance called Caffein, which is considered identical with thein. Coffee acts as a restorative after hard labor, seeming to retard the wastes of the tissues and food. It is used in the army (also in penitentiaries), not as a luxury, but as a matter of economy in the matter of food supply. Coffee, used to excess, frequently causes palpitation of the heart.

Malted and peptonized milk makes a valuable drink for invalids and dyspeptics.

Cocoa contains a stimulant called Theobromin. But unlike tea and coffee, cocoa and the preparation from cocoa known as chocolate are true foods, by virtue of the fat contained.

Beef tea and various beef extracts are very beneficial. There is not enough nourishment in them to maintain strength without other food. Their nutritive value has been somewhat over-estimated. Their value is as much, if not more, in their stimulating as in their nourishing effect. But many of the soups and drinks made from these preparations are very beneficial. They refresh the tired system wonderfully. Many a man who takes a drink of liquor to "brace him up," as he says, would do far better to take a cup of hot bouillon. $\mathrm{He}$ would find himself braced up for the time, without any bad reaction, or permanent injury to the system.

Alcohol. - Alcohol is not a food. But because it is taken into the digestive tube, and produces its effects primarily through the digestive system, it is here presented.

If we eat a sufficient amount of bread to-day, we do not crave a larger amount to-morrow; but the appetite for alcohol grows; the law of its use is the law of increase, until the terrible alcohol habit is formed. 
Aside from the fearful effects of the habitual use of alcohol upon the individual himself, statistics show that a large share of the poverty and crime in the world is due to its use. Nearly every child has known of the effects in the family of some drunkard, how the father is feared, how all are ashamed of him, how the children are poorly clothed, often not sent to school, because not sufficiently supplied with clothes and books; all these, and the dirt and misery so well known in so many cases, are a sufficient warning not to make the slightest beginning of this habit. History is full of accounts of men who thought they could stop when they chose; the grip of the alcohol habit is almost as relentless as the grip of death. There is one safe rule. "Touch not, taste not, handle not."

Some of the best authorities state that alcohol, taken in small doses, is oxidized in the body, producing energy; but they do not class it with foods, nor do they recommend its use.

As to its power to produce heat, the fact is that, as ordinarily taken, alcohol lowers the temperature of the body. It is well known that the face is flushed as the result of taking alcoholic drink. This means that more blood has been sent to the skin. This sending of blood to the skin gives a sensation of heat; we feel hot when the skin is flushed from other cause, as some emotion. But if the temperature of the body be taken at the time when the body feels warm, in each case it may be found that the temperature is actually lowered; and we can see the reason for this, for the more blood there is in the skin the more heat will be given off, and thus the amount of heat in the body diminished.

Neither does alcohol as usually taken increase the energy of the body so far as muscular work is concerned. Experience shows that men can endure more cold and more hard 
labor without alcohol than with it. This has been repeatedly proved in arctic expeditions, in the army and navy, during the hardships and exposures of forced marches and deprivations in all climates.

Especially if one is to be exposed to severe cold is it dangerous to take alcoholic drink; many a member of exploring parties has lost his life by disobeying this rule.

It is a significant fact that men training for athletic contests (no matter what their ordinary habits or principles) let alcoholic drinks alone. One of the famous pugilists said, "I'm no teetotaler, but when I have business on hand there's nothing like water and dumbbells."

"Alcohol is a typical stimulant; it acts as a whip, causing a temporary acceleration of physiological activity. Such acceleration must subsequently be paid for, the extra expenditure brought about by alcohol entailing diminished capacity for further exertion. Alcohol is thus of service only for emergencies of short duration; it is eminently harmful when prolonged exertion and endurance are required. Like all rapid stimulants, alcohol is in large doses a direct depressant." WALLER.

Many prefer to call alcohol a narcotic. In large doses it seems to paralyze the mechanism regulating the caliber of the arteries; hence the flushing above noted. Now, when the arteries are relaxed, the heart works harder to fill the greater space offered, and is thus overworked.

Alcohol has a strong affinity for water, and extracts it from tissues. When we preserve animal tissue in alcohol, the alcohol abstracts the water, thus hardening and preserving the substance.

Alcohol should be classed with the poisonous drugs (e.g., arsenic, chloroform, belladonna, strychnin, etc.), the exact nature of whose effects it is exceedingly difficult to determine. 
We do know that they are very dangerous substances; and there is one rule that will apply to them all: Never use them except under the advice of a physician.

"The most serious and widespread derangement of the natural taste is that caused by alcoholic drinks. Alcohol has been demonstrated to be a poison. Its continued use, even in what is ealled moderate quantities, will pave the way for many diseases, some of which are sure to overtake those who have the habit of using drinks with alcohol in them.

"Examples of the effect of the excessive use of alcoholic drinks are numerous and revolting enough in most communities to make the strongest appeals against their use.

"When it is seen that by the use of alcohol an intelligent man may act without reason; that a kind-hearted man may become brutal to his most loved friends; that it may cause an honorable man to become a dishonorable one; that it may make a noble nature become one with the most depraved of tastes; when its use has over and over again been the cause of disappointment, of intense suffering, and of crime, - it would seem that vastly stronger reasons existed against its use than the mere fact that some slight changes in the tissues occur which might possibly be demonstrated. It is to avoid these serious results that the use of alcohol is to be shunned, and not simply to avoid a differently shaped liver.

"The physiological effects of poisons are generally much greater than the visible changes which they produce in the tissues would lead us to expect. Indeed, such effects can seldom be detected by changes seen in the tissue cells.

"Strychnin produces powerful spasms which end in death. It acts, it is said, on the spinal cord, but it would be hard to show any changes that it produces in the cells. And a knowledge of the changes it produces in the cells could not make us fear the poison any more than we do, who know that it results in suffering and death." - JENkins. 
Many well-meaning persons use the various preparations called "root beers," perhaps without realizing that most, if not all, contain yeast, and in their preparation undergo fermentation, producing alcohol, though not ordinarily in large amounts. By giving such drinks (often called "temperance drinks") to children, an appetite for alcohol may be cultivated and the beginning of a terrible habit made. (And it may be well here to note the real meaning of the word habit, that which holds us.)

Nor is it advisable to keep cider about a house where there are children. "It is perfectly sweet," you say. Yes, but unless it is all soon consumed it will ferment. It is unwise, to say the least, to put temptation in the way of those whose habits are not formed.

\section{ALCOHOL IN THE BODY.}

[GrahaM Lusk, Ph.D., Professor of Physiology in the Yale Medical School, from the chapter on "The Chemistry of the Animal Body," in Howell's American Text-Bool of Physiology.]

"Alcohol in the stomach at first prevents the gelatinization necessary in proteid for peptic digestion; but this difficulty is of no great moment, because the absorption of alcohol is rapid and complete. It makes the mucous membrane hyperemic, promotes the absorption of accompanying substances (sugar, peptone, potassium iodide), and stimulates the flow of gastric juice. In this matter it acts as do other condiments (salt, pepper, mustard, peppermint), but if there be too great an irritation of the mucous membrane there is less activity (dyspepsia). The rapid absorption gives to alcohol its quick recuperative effect after collapse, and its value in administering drugs, especially antidotes. Alcoholic beverages, combining alcohol and flavor, promote gastric digestion and absorption, but often stimulate the appetite in excess of normal requirement. 
Alcohol is burned in the body, but may also be found in the breath, perspiration, urine, and milk. Alcohol has no effect on proteid decomposition, but acts to spare fat from combustion. The addition of 50 to 80 grams of alcohol to the food has no apparent effect on the nitrogenous equilibrium. Alcohol in the body acts as a paralyzant on certain portions of the brain, destroying the more delicate degrees of attention, judgment, and reflective thought, diminishing the sense of weariness (use after great exertion furnished to armies in the last hours of battle), and raising the self-esteem; it paralyzes the vasoconstrictor nerves, producing turgescence of the skin, with accompanying feeling of warmth, and thereby indirectly aiding the heart."

\section{STIMULANTS.}

[William H. Howell, Ph.D., M.D., Professor of Physiology, Johns Hopkins University, American Text-Book of Physiology.]

"The well-known stinulating effect of alcohol, tea, coffee, etc., is probably due to a specific action on the nervous system whereby the irritability of the tissue is increased. The physiological effect of tea, coffee, and chocolate is due to the alkaloid caffeine (trimethyl xanthin) and theobromine (dimethyl xanthin). In small doses these substances are oxidized in the body and yield a corresponding amount of energy, but their value from this stand point is altogether unimportant compared with their action as stimulants. Alcohol also, when not taken in too large quantities, may be oxidized in the body, and furnish a not inconsiderable amount of energy. It is, however, a matter of controversy at present whether alcohol in small doses can be considered a true foodstuff, capable of serving as a direct source of energy, and of replacing a corresponding amount of fats or of carbohydrates in the daily diet. The evidence is partly for and partly against such a use of alcohol. 
When alcohol is taken in excess it produces the familiar symptoms of intoxication, which may pass subsequently into a condition of stupor or even death, provided the quantity taken is sufficiently great. So, also, the long-continued use of alcohol in large quantities is known to produce serious lesions of the stomach, liver, nerves, blood-vessels, and other organs. The effect of alcohol upon the body evidently varies greatly with the quantity used."

\section{EFFECT OF ALCOHOL ON MOUNTAIN CLIMBERS.}

[From The Nation.]

"Dr. Otto Snell issued a card requesting mountain climbers to forward their personal experiences and views to him. He received sixty communications, thirty-seven of which, or $62 \%$, condemn the use of liquors, wine, or beer, as an impediment rather than an aid. Twelve are for a moderate use of wine, but pronounce against brandy and beer. Three believe in taking brandy along, to be used, however, not as a stimulant, but in case of need as a medicine or to mix with glacier water. Only five of the sixty expressed their belief that alcoholic drinks are beneficial or harmless to climbers. The general conclusion drawn by Dr. Snell from these answers is that, while in exceptional cases alcohol may be harmless, or possibly useful, as a rule great moderation is desirable; while the majority of experts are for total abstinence until after the climb is over, and some even strongly urge abstinence, or great moderation, on the day before the expedition. One of the correspondents expressed his conviction that the bottled drinks taken along by climbers benefit no one but the tavernkeepers from whom they buy them."

In August, 1897, the writer climbed Pike's Peak, and spent the night there. The keeper of the house there testified that those who took alcoholic drink for the relief of giddiness, 
nausea, or other distress that often is felt as a result of the effort of climbing in such altitude, - that almost invariably such symptoms were aggravated instead of being relieved. Dr. Hodge, in an article in Popular Science Monthly (March and April, 1897), entitled "Experiments on the Physiology of Alcohol," makes the following quotations, "Hemholtz has said, in describing his methods of work, that slight indulgence in alcoholic drinks dispelled instantly his best ideas. Professor Gaule once told the writer, as an experiment during the strain of his 'Staatsexamen,' that he suddenly stopped his wine and beer, and was surprised to find how much better he could work. An eminent professor in Leipsic once said that the German students could do 'twice as much work' if they would let their beer alone. Dr. August Smith has found that moderate non-intoxicant doses of alcohol (forty to eighty cubic centimeters daily) lowered psychic ability to memorize as much as seventy per cent."

\section{[From Thомpsox's Practical Dietetics.]}

"... the following general propositions comprise the belief of many authorities who have devoted careful research to this exceedingly important topic :-

" 1 . The use of alcohol in any shape is wholly unnecessary for the use of the human organism in health. It does not exist as a natural product. The very lowest types of man Australian and many Polynesian savages - know nothing of it, and drink only water and fresh fruit juice, such as that of the cocoanut, although they speedily acquire a fondness for alcohol when it is given them.

" 2 . A large number of persons are undoubtedly better without alcohol, and may prolong their lives by total abstinence.

"3. The lifelong use of alcohol in moderation, as an occasional beverage with meals, does not necessarily shorten the duration of life or induce disease in some persons, while in 
others it undoubtedly produces gradual and permanent changes, chiefly of a cirrhotic character, in the blood-vessels and viscera, such as the liver and kidneys. These alterations, which may be slow and subtile in character, may not in themselves materially impair the health or cause an ultimately fatal result; but they tend to weaken vital organs and produce a condition of premature senility, so that if the patient be overtaken by any severe disease, as, for example, by an acute infection like pneumonia, or a chronic one like pulmonary tuberculosis, the resistance of the body to the force of the disease is materially impaired, and the danger to the patient is seriously enhanced.

"4. There are many persons whose constitutional inheritance is such that they should be particularly warned against the use of alcohol; and in some such cases, as, for example, among those who are subjects of well-marked gouty diathesis, it is better that the use of alcohol should be imperatively forbidden.

"5. The abuse of alcoholic stimulation is invariably injurious, although the extent to which evil influences become manifest depends upon the constitution of the individual, in connection with the two factors of heredity and environment.

"6. There are a number of diseases in which the temporary use of alcohol is of positive service, and there are a number of cases in which it becomes a necessity in order to prolong life.

“7. In many cases of malnutrition and malassimilation of food, alcohol is itself a food; and its consumption under proper direction results in an increase of body weight and strength, and inprovement of functional activity. These results are accomplished in part through the action of the alcohol as a definite food, and in part through its remarkable effect in force production. The latter is due to its own direct combustion, by which in chronic diseases and in critical, acute, and exhausting affections it spares that of the tissues of the body. 
"Although alcohol is such a strong force producer and heat generator, its effect in this direction is very soon counterbalanced by its stronger influence in lowering the general tone of the nervous system, and in producing positive degeneration in the tissues. In the condition of health more food is usually eaten and more force is developed than are actually necessary for the body, and there is constantly a reserve supply of energy on hand which may be utilized for any extraordinary exertion; and hence the constant use of alcohol as a food or stimulant in health is both unnecessary and unadvisable. When alcohol is consumed in health in addition to a normal or excessive quantity of solid food, by its more ready combustion it prevents the complete oxidation of the latter, and favors the accumulation of suboxidized waste products, which are always harmful in the system. Excesses in eating are thus doubly aggravated by the effects of alcohol. It is the almost universal testimony of army surgeons, and the experience of those who, like Greely, Stanley, and others, have led long and perilous exploring expeditions involving great fatigue and unusual endurance, that muscular overwork and climatic hardships are much better endured if alcohol is entirely abstained from.

"It has always been found in armies, that when good food was at hand the issue of alcohol with the regular ration produced an increased percentage of sick days and of incapacity for work. Col. A. A. Woodhull, surgeon U. S. army, writes me: 'I do not think that any of our medical officers would seriously advocate the issue of alcohol as a measure of health; but I believe that its habitual use during the Rebellion was prohibited for reasons of discipline, while it still might have been occasionally issued as if for health. On the rare occasions when it might serve a good purpose, as a temporary stimulant after a long and wet march, the wagons would be in the rear, owing to the same conditions that fatigued the men.' 
“While all this applies to prolonged effort of any kind, and to conditions where other food can be obtained and assimilated, it does not detract from the fact that alcohol is a most helpful food and stimulant in emergencies, when other food cannot be had, or when the body is temporarily endangered from acute disease and the higher rate of combustion in fever, or from failure to assimilate other nourishment.

"Captain Woodruff, assistant surgeon United States army, says, "Spirits can never be used in the army as a regular issue; the practice is thoroughly vicious, and was virtually abandoned sixty years ago. On extraordinary occasions of great fatigue they are allowable in moderation. Under such temporary stimulation the men will brace up and perform the necessary work of making earthworks, etc., when without it they would be too exhausted to do anything. Without such stimulation a man is not worth much after he has made a forced march of forty miles.'

"The problem whether the world as a whole is better or worse for the existence of alcohol, aside from all ethical questions, and viewed merely from the scientific standpoint of the influence of alcohol upon mortality, is difficult of solution; for to offset the numerous cases of fatal alcoholism, and the still larger number of cases of diseases which would not presumably be fatal without the existing condition of chronic alcoholic poisoning of the system, are very many cases, among both infants and adults, in which life is undoubtedly saved by the prompt resort to this food and stimulant, and its energetic use. So long as man is exposed to hardships and conditions arising from improper and deficient food supply, as well as to the numerous infectious diseases to which he is heir, alcohol must still be regarded rather as a blessing than a curse; for there is no form of stimulant and food combined, or stimulant alone, which, taken all in all, can be so completely relied upon 
in cases of emergency. Alcohol when taken alone will prolong life beyond the period at which it terminates from starvation."

\section{BEVERAGES CONTAINING ALCOHOL.}

[Rонє, Text-Book of Hygiene.]

"The physiological action of alcohol has been pretty fully worked out by Binz and his pupils, and by other experimenters. From these researches it appears that the first effect of taking alcohol, sufficiently diluted, into the stomach is to increase the flow of saliva and gastric juice. This effect is probably reflex, and results from a stimulation of nerve terminations in the stomach. The alcohol is rapidly absorbed, and is carried in the blood, without undergoing chemical change, to the nervous centers, lungs, and tissues generally. In the brain the alcohol probably enters into combination with the nervous tissue, modifying the normal activity of the various centers, either increasing the activity, if the alcohol is in small quantity (stimulating effect), or diminishing it, if in larger quantity (depressing effect), or entirely suspending the activity of the center's, if in sufficiently large quantity (paralyzing effect).

"Alcohol stimulates the vasodilator nerves, causing dilatation of the smaller vessels; in consequence of this the blood is largely sent to the periphery of the body, the blood-pressure diminishes, and heat radiation is increased. At the same time a portion of the alcohol is used up in the production of animal heat, thus economizing the expenditure of fats and proteids, and acting as a true respiratory food. Alcohol does not contribute nutritive material to the body; it only permits that which is stored up to be saved for other uses, by furnishing easily oxidizable (combustible) material for carrying on the respiratory process and supplying animal heat. 
"During the use of alcohol the excretion of urea is diminished. This shows that waste of tissue is retarded in the body.

"Regarding the statement of some authorities that alcohol does not undergo any change in the body, but is excreted unchanged, Binz asserts that alcohol appears in the urine only when exceptionally large quantities have been taken, and then in very small proportion. It is not excreted by the lungs, the peculiar odor of the breath being due not to the alcohol, but to the volatile aromatic ether, which is oxidized with greater difficulty, and so escapes unchanged.

"While alcohol produces subjectively an agreeable sensation of warmth in the stomach and on the surface of the body, the bodily temperature is not raised. The subjective sensation is due to the dilatation of the blood-vessels and the sudden hyperæmia of those parts.

"During fevers and other exhausting diseases alcohol is invaluable to prevent waste of tissue and sustain the strength. It does not act merely as a stimulant to the circulation and nervous system, but, as above pointed out, saves the more stable compounds by furnishing a readily oxidizable respiratory food.

"When taken in small doses by healthy persons alcohol diminishes the temperature by increasing heat radiation. When large quantities are taken the bodily temperature is reduced by diminishing heat production, as well as by increased radiation. This is shown in the condition known as deaddrunkenness, in which the temperature is sometimes depressed as much as $20^{\circ} \mathrm{F}$. below the normal. Cases in which the temperature sank to $75^{\circ}, 78.8^{\circ}$, and $83^{\circ} \mathrm{F}$. have been reported, with recovery in all cases.

"The constant use of alcohol produces in all the organs an excess of connective tissue, followed by fatty degeneration and the condition known as cirrhosis. The organs most frequently 
affected are the stomach, liver, and kidneys. Serious pathological alterations also occur in the circulatory, respiratory, and nervous systems.

"Alcohol is not necessary to persons in good health. Probably most persons, regardless of their state of health, do better without it. Its habitual use, in the form of strong liquors, is to be unreservedly condemned. The lighter wines and malt liquors, if obtained pure, may be consumed in moderate quantities without ill effects. Even in these forms, however, the use of alcohol should be discouraged, or perhaps prohibited, in the young.

"Neither in hot nor in cold climates is alcohol necessary to the preservation of health, and its moderate use even produces more injury than benefit. The polar voyager and the East India merchant are alike better off without alcohol than with it.

"It has long been a prevalent belief that the use of alcohol enables persons to withstand fatigue better than where no alcohol is used. A large amount of concurrent testimony absolutely negatives this belief.

"The predisposition to many diseases is greatly increased by the habitual use of alcohol. Sunstroke, the acute infectious diseases, and many local organic affections, attack, by preference, the intemperate. A recent collective investigation by the British Medical Association brought out the fact that croupous pneumonia is vastly more fatal among the intemperate than among those who abstained from the use of alcoholic liquors.

"A further investigation by Baer has shown that the average expectation of life among users and dealers in alcoholic liquors is very much shortened. The following table gives a comparative view of the expectation of life in those who abstained from and those who used alcohol:- 


\section{EXPECTATION OF LIFE.}

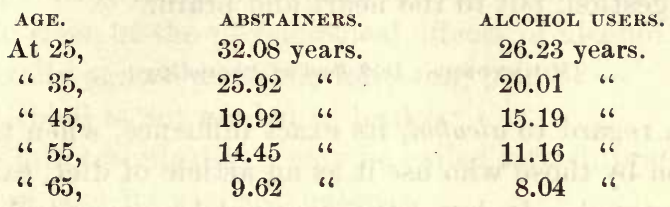

TABLE SHOWING THE INFLUENCE OF ALCOHOL UPON THE MORTALITY FROM VARIOUS DISEASES.

GENERAL MALE POPULATION. ALCOHOL VENDERS.

$\begin{array}{lrlrc}\text { Brain disease, } & 11.77 \text { per cent. } & 14.43 \text { per cent } \\ \text { Tuberculosis, } & 30.36 & \text { "6 } & 36.57 & \text { " } \\ \text { Pneumonia and pleuritis, } & 9.63 & \text { "6 } & 11.44 & \text { "6 } \\ \text { Heart disease, } & 1.46 & \text { " } & 3.29 & \text { " } \\ \text { Kidney disease, } & 1.40 & \text { "6 } & 2.11 & \text { " } \\ \text { Suicide, } & 2.99 & \text { "6 } & 4.02 & \text { " } \\ \text { Cancer, } & 2.49 & \text { "6 } & 3.70 & \text { " } \\ \text { Old age, } & 22.49 & \text { "6 } & 7.05 & \end{array}$

\section{ACCESSORIES TO FOOD.}

[Halliburton, Text-Book of Chemical Physiology and Pathology.]

"Alcohol. - Small quantities of the alcohol taken leave the body by the breath and urine as such, the greater amount is decomposed into simpler products (acetic, oxalic, carbonic acids, and water); the formation of these must give rise to a certain amount of bodily heat. It has been calculated that a man can burn off in his body two ounces of absolute alcohol daily. Alcohol is thus, within narrow limits, a food. It, however, lessens proteid metabolism by about 6 per cent, and thus ultimately leads to a diminution of the heat produced in the body. It is, moreover, a very uneconomical food; much more nutriment would have been obtainable from the barley or the grapes from which it was made. The value of alcohol used 
within moderate limits is not as a food, but as a stimulant not only to digestion, but to the heart and brain."

\section{[M'KENDRICK, Text-Book of Physiology.]}

"With regard to alcohol, its exact influence, when taken in moderation by those who use it as an article of diet, cannot be precisely stated. It has been asserted by several observers that alcohol is eliminated from the body as alcohol by the various excretory channels. The evidence of this is doubtful, and it is probable that it is split up into simpler compounds. ... A small part of the alcohol ingested no doubt is exhaled by the mucous membrane of the lungs and by the kidneys. The odor of the breath depends on the elimination of oxidation products, such as fusel oil. If oxidized even to a small extent, and the evidence, as already indicated, points to the oxidation of by far the larger proportion of it (95 per cent), alcohol must be regarded, in the scientific sense, as a food. No doubt also its ingestion diminishes the metabolism of proteids to the extent of about 6 per cent, as shown by the diminished excretion of urea. Its oxidation will also be attended by the production of heat; but as, on the other hand, it lessens the production of heat by interfering with the metabolism in proteid tissues, and also by diminishing the oxidation of carbohydrates and fats, the final result is an actual diminution of bodily temperature. While, therefore, alcohol must be classed technically as a food, it is in many respects an unsuitable food, and its place can be taken with great advantage by other substances. In small doses it acts as a local excitant of the digestive mucous membrane, and afterwards as a diffusible stimulant upon the circulation and central nervous system. In some cases it may aid the digestive process; but in a state of health it is not only not required, but its use, except in small doses, is positively prejudicial." 
These various authorities have thus been freely quoted, to show that while there is considerable divergence of opinion in regard to some of the physiological effects of alcohol, they are substantially agreed as to the following points :-

1. Alcohol is not needed in health.

2. While technically it may be called a food, practically it is a poison, and its use is dangerous.

The danger is especially great where there is a latent hereditary tendency to inebriety or insanity. The danger is also very great when the disease for which alcohol is prescribed is accompanied by melancholy and depression. Many individuals, on finding a drug which exhilarates and banishes the weight of oppression by which they are borne down, are tempted beyond their power of resistance, even though the reaction brings them into a worse condition than the one from which they sought relief.

The pressure of modern life, and the intensity of the struggle for a living, brings about a condition of nervous strain that is fraught with great danger. Every thinking man should see that to use alcoholic drink for the relief of such a condition is like venturing out in a boat above the Falls of Niagara, - he knows not when the rushing, mighty power will gain the mastery and dash him to destruction.

Tobacco. - The use of tobacco is needless. Man gets along well enough without it. It is injurious to many. It is an expensive habit. Many a man spends enough on tobacco to send a boy through college. With the excellent cheap printing of to-day, many of the very best books may be bought for the money that is paid for as many cigars. Even for those who can abundantly afford it, it seems extremely selfish, when it is needless, and there is so much good that might be done with the money. Another very selfish feature is, that so many men do not seem to consider the fact that the air is public prop- 
erty, and they have no right to fill the air with any gas or smoke that is offensive to others. Very likely many men derive great comfort from the use of tobacco after they have once formed the habit; but most of these were made sick in learning, showing that the use is unnatural.

It seems to be clearly proved that cigarette smoking is very injurious, especially to boys. And if men smoke cigars, the example is set for the boys to smoke cigarettes. Many of the cigarettes are said to be steeped in preparations of opium; so that the use of cigarettes is often subjecting the user, not only to the tryanny of tobacco, but to that of opium as well.

Perhaps Robinson Crusoe might have been excused for using tobacco, having no one to save money for, no unfortunates to aid, no children to educate, no one to whom he might set a bad example, no one whose breath of air he could contaminate, no one to smell his breath, no one to see the offensive results. But a man living in the society of so many to whom this habit, in all its features, is so disgusting and in every way offensive, ought seriously to consider whether he is doing right in continuing such a practice.

Many boys seem to think it is manly; they wish to do as others do. It is not manly to imitate any one. Do nothing simply because some one else does it. To do this is to be a slave, to be led. And one bad feature of the tobacco habit is that one makes himself a slave to the weed. For, like other narcotics, it has a powerful influence on the system; and the habit, once formed, is hard to break.

How many men have been heard to say, "I wish I had never formed the habit."

Has any one in middle or later life ever been heard to say, "I wish I had formed this habit"?

Cooking. - Cooking is designed to make food more palatable and more digestible. Some foods, such as eggs, are as 
digestible before they are cooked as after, often more so, as they are very frequently badly cooked. But many foods in the raw state are unattractive, or even repellent, whereas cooking usually develops an agreeable odor and taste. Cooking should soften the harder and tougher tissues, such as cellulose in vegetables and the connective tissue of animal foods.

If meat be put into cold water and the water gradually warmed, the soluble material of the meat may be extracted; and this is the principle followed in making soups. But if we wish to cook the meat itself, the juices should be retained instead of withdrawn. For this purpose boiling water is poured over the meat to coagulate the outer layer, and prevent the extraction of the juices. The same principle applies to baking and roasting and also to broiling. The outside is subjected to high heat at the beginning of the cooking, which forms a layer nearly impervious to the nutritious material inside. In these modes of cooking, it is often very desirable to reduce the heat applied after the first few minutes, so that the interior may be more gradually cooked; this is, perhaps, especially true in broiling.

Frying, as ordinarily done, is not a good mode of cooking, in fact, is often very bad, as the food is frequently penetrated with fat, and rendered very indigestible. But true frying, that is, by immersion in boiling fat, is a good mode of cooking. This coagulates the albuminous substance on the outside, keeps in the nutritious juices, and prevents soaking with the fat. Often the food to be thus cooked is first coated with white of egg, which is very quickly coagulated, and helps form a protecting outside crust.

Cooking starch causes the starch grains to swell and burst, and makes the starch much more digestible.

(For further information read, in the Appendix, the extended quotations from Mrs. Abel.) 


\section{THE DIGESTIVE SYSTEM.}

The tissues are worn out by their oxidations. They are built up again by the blood, and the blood is renewed by the food.

All food must be reduced to the liquid condition, if it is not already liquid.

The chief organ in this work of liquefying the food is the Digestive Tube, or "alimentary canal" as it is called. As the food passes through the digestive tube, it is subjected to various mechanical and chemical processes, which liquefy it, and bring it into such a condition that it can be absorbed by the mucous lining of the digestive tube and passed into the blood.

To take a special instance, a muscle is in part worn out by the oxidation during its activity; to replace the loss suppose we take a piece of steak. We cannot substitute this directly in the place of the worn-out tissue. In digesting the steak we must tear it all to pieces, and reduce it to a liquid form by the action of the teeth and by the various liquids from the glands along the digestive tube. In short, the muscle, as such, must be thoroughly destroyed; in the liquid produced by the digestion of the beef, there is no trace whatever of the structure of the beef. But the blood, taking this material, builds muscle which can hardly, if at all, be distinguished from the original beef.

If the food taken be all ready to build tissue, for example, certain forms of sugar, liquid, soluble, and of the proper chemical composition, it will not need to go through these changes.

In order to understand the process of digestion, let us first turn our attention to the anatomy of the organs of digestion. 


\section{Organs of the Abdominal Cavity. - (Rabbit or cat.)}

1. Slit the skin in the middle line from the breast-bone to the pelvis, and strip it well back to the sides. Observe the thin Abdominal Museles, which form the ventral wall of the abdomen. Carefully slit this in the middle line from the pelvis to the breast-bone; from the middle of this slit cut outward on each side, and turn back the flaps.

2. The lining of the abdomen is the Peritoneum; draw the tips of the fingers over it to learn its smoothness.

3. Observe the coiled Intestine, noting any variations in size, shape, or markings, but do not now move any part from its natural position. In a recently killed animal the intestines usually show a slow motion, like that of a mass of earthworms, coiled and crawling over each other. This is the Peristaltic motion, due to the shortening and relaxation of the muscles in the walls of the intestines. This is always a wave-like motion, proceeding from the anterior toward the posterior end of the intestine.

4. In the anterior part of the abdomen the dark-colored Liver may be seen, overlapping the Stomach; and in the hinder part of the abdomen the Bladder may be seen, varying greatly in size and appearance according to the state of distention.

5. Pull the intestine backward, and make out the shape, size, position, and color of the stomach. Care must be taken in handling the liver. Its tissue is so delicate that it is easily ruptured; and the organ is so full of blood that enough may soon escape to make the dissection unsightly, and to obscure some of the organs. Do not touch the liver with the dissecting instruments, not even with the handle of the scalpel; and in handling it with the fingers, take care not to lacerate it with the nails. Observe how the liver and stomach fit together; push the liver forward, and turn the stomach back to find a white tube entering its anterior surface; this is 
the gullet, or Esophagus. At the larger end (to the left) of the stomach is a deep red body, the Spleen.

6. Find now the connection between the stomach and the intestine. Make a drawing of the stomach, showing its shape and the connection with the gullet and the intestine.

7. Trace the intestine; that part which forms a long loop near the stomach is the Duodenum. Within this loop is an irregular, pinkish, or fatty-looking mass, the Pancreas. Find the Pancreatic Duct entering the intestine. This is more easily found in the dog.

8. Observe that the intestine is held by a thin membrane, in which are branching blood tubes; this is the Mesentery; find its supporting attachment. In tracing its course, drag the intestine out of the abdominal cavity, but do not tear the mesentery.

9. Observe that the Small Intestine runs into the Large Intestine at a right angle near the beginning of the large intestine. The blind end of the large intestine, back of the entrance of the small intestine, is the Cecum. In the rabbit it is long, in the cat short.

10. Turn the liver forward, and find, on its posterior surface, the dark Bile-sac. The Bile-duct, by which the bile is conveyed into the intestine, may be traced by cutting into the bile-sac, and probing with a bristle tipped with sealing-wax.

11. Pull the liver back, and examine the thin muscular partition, the Diaphragm, which extends across the body, separating the chest cavity, or thoracic cavity, from the abdominal cavity.

12. Note the passage of the gullet, aorta, and postcaval vein through the diaphragm.

13. Attached to the dorsal walls of the abdomen are the dark-colored, bean-shaped Kidneys.

Tie the gullet in two places half an inch apart, and cut 
through between them. Do the same with the hinder part of the large intestine, the Rectum, and sever it. Remove the stomach and intestines, carefully cutting the mesentery along the whole attachment to the intestines, and uncoil the latter. How many times is the length of the body, including the head, contained in the length of the intestine? Compare the lengths of the small intestine, cecum, and large intestine. Cut out about an inch of the sinall intestine in the middle of its course, slit it open lengthwise, wash it thoroughly, and with a lens examine its inner surface under water, to see the thread-like projections, or Villi. In the same way examine a piece of the large intestine. These points may be made out in the intestine of a dog, or, still better, from specimens of the calf's intestine obtained from the butcher.

To illustrate the relation of the mesentery to the intestine, suspend the arm in a sling made of a handkerchief; press the two thicknesses of the cloth together just above the arm to represent the two layers of the mesentery. A more

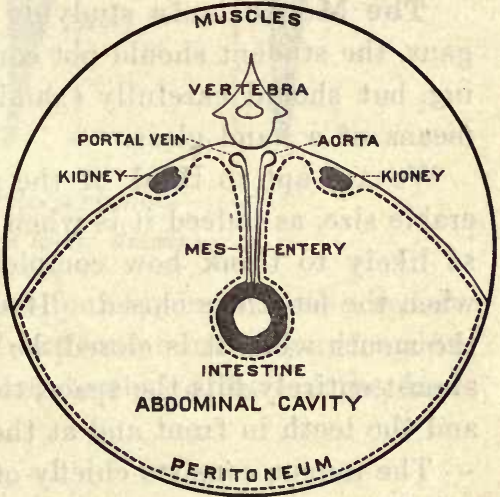

Fig. 55. Cross Section of Abdomen. complete representation may be made as follows: Material: piece of large (one inch or more in diameter) rubber tubing, eight inches long; sheet of thin white court-plaster, six inches by twelve inches; red, blue, and white cord; lay the tube across the middle of the court-plaster; gum the plaster snugly around the tube; between the two adjacent layers of the 
court-plaster, where they meet after passing around the tube, lay the three kinds of cord, each frayed out at one end, the frayed ends resting upon the tube. Moisten the court-plaster, and press the layers firmly together. The court-plaster should now adhere so closely to the tube as hardly to be seen; and the two layers should seem as one, in which appear the cords representing the arteries, veins, and lacteals.

The Organs of Digestion. - The organs of digestion are the digestive tube and the accessory parts, the masticatory organs, the glands in, and alongside of, the walls of the tube.

The parts of the digestive tube are the mouth, the pharynx, the gullet (or esophagus), the stomach, the small intestine, the large intestine.

The Mouth. - In studying the mouth and contained organs, the student should not content himself with mere reading, but should carefully examine his own mouth cavity by means of a hand glass.

We are apt to think of the mouth as a cavity of considerable size, as indeed it is when fully opened; but we are not so likely to think how completely the cavity is obliterated when the mouth is closed. If one notes the sensations from the mouth when it is closed, he will perceive that the tongue almost entirely fills the space, touching the roof of the mouth, and the teeth in front and at the sides.

The tongue consists chiefly of muscles, extending in different directions, thus giving the tongue a variety of motions. The tongue is the chief organ of taste, and is therefore (with the sense of smell) the gatekeeper of the digestive tube. The tongue has also a keen sense of touch (the keenest of any part of the body), and so is useful in detecting and removing any food particles that may remain on the teeth after a meal. During mastication the tongue, with the lips and cheek, keep the food between the teeth. When the morsel of food is 
sufficiently masticated, the tongue pushes it back into the pharynx to be swallowed.

The Teeth. - The teacher can easily get a lot of teeth from almost any dentist for the asking. These should be cleaned before using them in the elass. Use pearline or any washing-soda. If there be enough time, let each pupil make
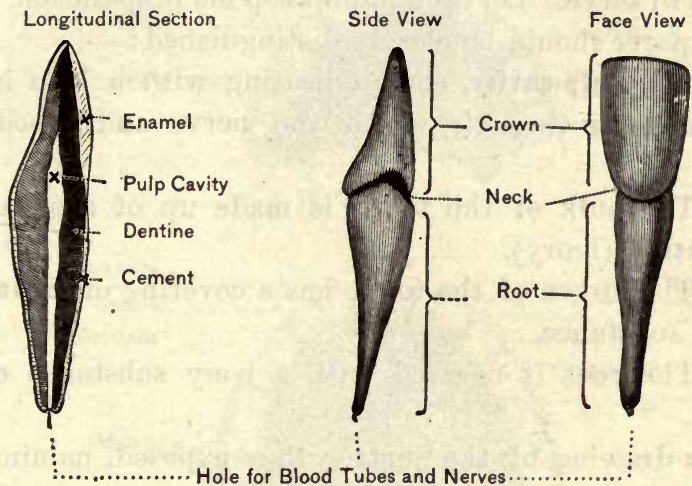

Fig. 56. Parts of a Tooth. (Incisor.)

a drawing of one of each of the four kinds of teeth; and it would be well to draw both a front (outer surface) and a side view (surface adjacent to another tooth), of each of the four kinds.

The study of a single tooth.

1. External features of a tooth.

Examine one of the front teeth. It has the following parts ·

1. The Crown, the part that is above the gum.

2. The Root, the part that was buried beneath the gum.

3. The Neck, a more or less constricted part, dividing the crown from the root; it is normally at about the surface of the gum.

4. A Hole at the tip of the root. 
2. Structure of a tooth.

Let each pupil prepare a longitudinal section of a tonth as follows: Imbed a tooth in a little sealing-wax on the end of a spool, cork, or block of wood. With a grindstone grind away one half, showing the, pulp cavity to the tip of the root as in Fig. 56. If human teeth cannot be obtained, almost any kind will serve. Let each pupil keep his preparation. The following parts should be clearly distinguished:-

1. The Pulp-cavity, communicating with a hole in the tip of the root, through which the nerve and blood-tube entered.

2. The bulk of the tooth is made up of a substance called Dentine (ivory).

3. The crown of the tooth has a covering of Enamel, a very hard substance.

4. The root is covered with a bony substance called

\section{Cement.}

Make a drawing of the surface thus exposed, naming the parts.

The Kinds of Teeth and their Arrangement. - Beginning at the middle of the front of the mouth, there are (in the normal adult) eight teeth in each half-jaw : two Incisors, one Canine, two Bicuspids (or Premolars), and three Molars.

The kinds and arrangement of teeth are often expressed by a Dental Formula, in which the numerators indicate the upper jaw and the denominators the lower, thus: $\mathrm{I}_{\frac{2}{2}}, \mathrm{C}_{\frac{1}{1}}$, $\mathrm{PM}_{2}, \mathbf{M}_{3}^{3}$ (for one side of the head).

The crown of an incisor is chisel-shaped; but the root is flattened in the opposite direction, i.e., at right angles to the jaw, instead of parallel to it, as is the case with the crown. If possible, have at hand also a skuli from which the teeth have been extracted, in order to see the cavities into which the teeth fitted. 
The canine tooth has a conical crowi, and a longer root than the incisor.

The bicuspid has two points.

The molar has a cuboidal crown, and usually two or three roots.
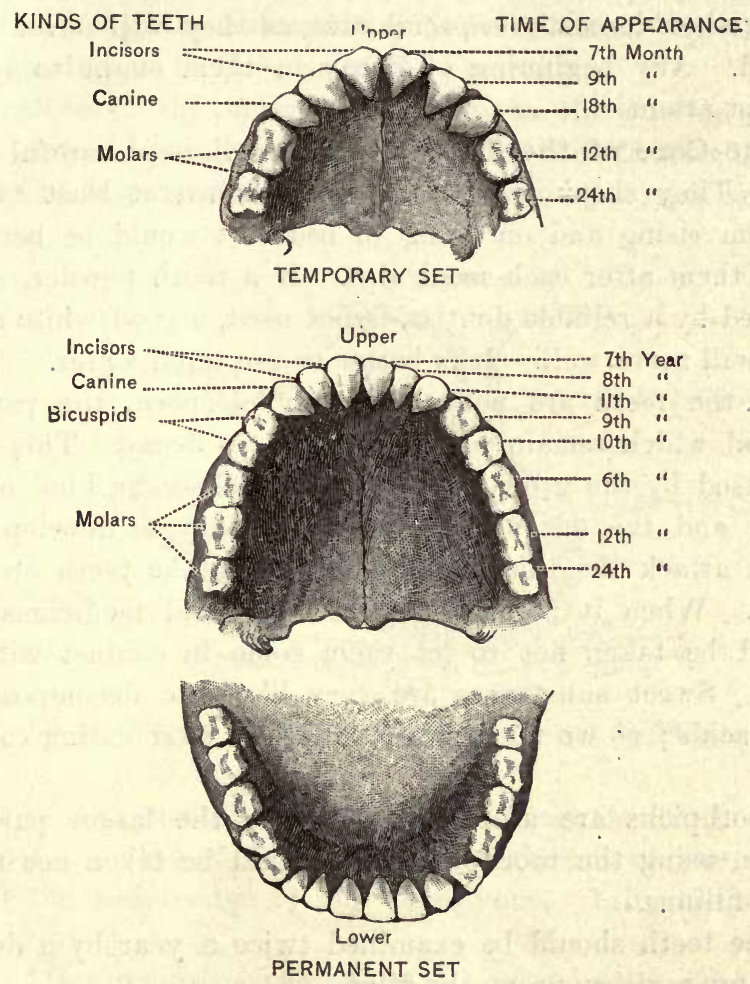

Fig. 57. TEETH : Kinds, Arrangements, and Times of Appearance.

The thirty-two teeth of the permanent set were preceded by a temporary set of twenty Milk Teeth.

Because the first set is temporary, it should not therefore 
be neglected. Cavities in these should be filled and the teeth kept clean.

Before the temporary set has gone, the first of the permanent set appear. The first of these, often called the "sixyear molars," are just back of the hindermost " milk molars." These should receive especial care, as they will never be replaced. Any beginning of decay in them ought to receive prompt attention.

The Care of the Teeth. - The teeth need careful attention. They should be thoroughly brushed at least twice a day, on rising and on going to bed. It would be better to clean them after each meal also. If a tooth powder, recommended by a reliable dentist, is not used, a good white castile soap will serve well. It is better to use tepid water.

If the teeth are not thoroughly cleansed, the particles of food which remain will soon begin to decay. This decay is caused by the growth of germs, usually some kind of bacteria; and the decay thus begun is likely to develop acids which attack the limy material of which the teeth are composed. When it is necessary to take acid medicines, care should be taken not to let them come in contact with the teeth. Sweet substances are very likely to decompose and form acids; so we must clean the teeth after eating candies, etc.

Toothpicks are useful in removing the larger particles. But in using the toothpick care should be taken not to dislodge fillings.

The teeth should be examined twice a year by a dentist, and any cayities promptly filled.

Dissection of the Head of the Rabbit. - Remove the skin from the head.

1. Below and back of the ear is an irregular pink mass, the Parotid Salivary Gland. The duct which conveys its secre- 
tion runs forward over the cheek and opens on the inside of the cheek. It is hard to trace in the rabbit. Find it in the dog, slit into it with fine scissors, and push a black bristle forward through it to find its opening in the mouth.

2. Just back of the angle of the lower jaw observe a roundish body, the Submaxillary Salivary Gland. In the dog trace its duct as in the case of the parotid.

3. Observe the muscle which covers the outside of the back part of each lower jaw. This is the Masseter Musele. Place the fingers on the angle of your own jaw, and note the

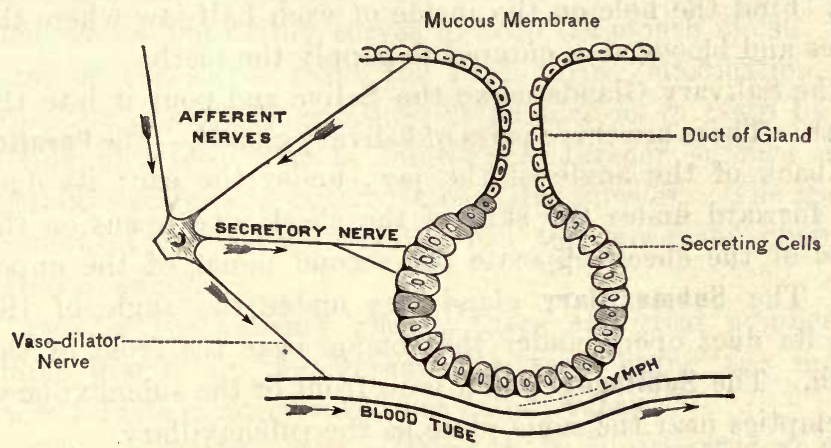

Fig. 58. Diagram of a Salivary Gland. (After Landois \& Stirling.)

action of the masseter muscle in shutting the teeth firmly together. In the rabbit note the attachinent of the masseter to the under edge of the cheek-bone. Trim the muscle entirely away.

4. After removing the submaxillary glands a muscle will be found on each side, having its origin on the inside of each half-jaw near their junction. These are the Digastric Muscles; they depress the lower jaw. Cut away all the muscles and other connection, and remove the whole of the lower jaw. 
5. In the human skull study the joint by which the lower jaw is articulated.

6. The masseter muscle passes from the angle of the jaw to the lower border of the bridge, or arch, of the cheekbone.

7. The temporal muscle is attached to the thin wing or process of the jaw in front of the hinge, and passes up inside of the arch of the cheek-bone to spread over the temple. Place the tips of the fingers on the temples, and shut the teeth firmly together. The hardening of the temporal muscle is felt.

8. Find the hole on the inside of each half-jaw where the nerves and blood-tubes entered to supply the teeth.

The Salivary Glands make the Saliva and pour it into the mouth. There are three pairs of salivary glands,- the Parotid, just back of the angle of the jaw, under the ear; its duct runs forward under the skin of the cheek, and opens on the inside of the cheek opposite the second molar of the upper jaw. The Submaxillary gland lies under the angle of the jaw ; its duct opens under the tongue near the front of the mouth. The Sublingual gland is in front of the submaxillary, and empties near the same place as the submaxillary.

Action of the Salivary Glands. - The salivary glands pour into the mouth a liquid which they manufacture from materials taken from the blood. In structure the gland may be compared to a bunch of grapes, the grapes representing the little cavities, with a wall of cells which make the saliva; from these cavities the liquid passes into the individual duct, represented by the stem of a single grape; many of these uniting form the main stem, which corresponds to the main duct. A rich network of eapillaries surrounds the gland; when the gland is at work it receives more blood; the liquid part of the blood (plasma) soaks out through the capillary 
walls and surrounds the gland; it is now called lymph; from the lymph the gland directly gets its material.

The glands are doubly dependent on nerve control :-

1. Through the control of the arterial muscles by the nerves the amount of blood sent to the glands is regulated.

2. Nerves also go to the cells of the gland to control their activity. When we taste, smell, see, or even when we think of, some delicious food the mouth may "water," as we say; i.e., the salivary glands are, by reflex action, stimulated to activity; on the other hand, some emotions, such as fear, check the flow of saliva. The saliva is mostly water, and, when we are not eating, serves to keep the mouth moist. The water of the saliva soaks the food during mastication, and helps the process of grinding; it enables us to taste by dissolving any food that is soluble; it further enables us to swallow what would otherwise be a dry powder. The special element of the saliva, Ptyalin, has the power of changing starch to sugar.

Besides the salivary glands, there are great numbers of simple glands in the Mucous Membrane lining the mouth. These secrete a glairy substance called Mucus.

The amount of saliva secreted daily is estimated at three pints. Of course the glands should be allowed to rest between meals. The habit of chewing gum, though supposed to aid digestion, undoubtedly does far more harm than good. During the resting period the glands accumulate material for the active work of secretion; for there is no sac in which to store the saliva, and it must be made as fast as it is needed.

"The character of action of salivary ferment is further defined by experiments showing: 1, that it is destroyed by boiling; 2, that its action is delayed or suspended at a low temperature, most pronounced at about body temperature $\left(37^{\circ} \mathrm{C}.\right) ; 3$, that it acts best in a neutral or in a faintly alka- 
line medium, not at all in an acid medium, or in too strong an alkaline medium; 4, that it has almost indefinite power, if the product of its own action (sugar) is not suffered to accumulate. In all these respects, with the exception of the third, the salivary ferment resembles ferments in general, which are destroyed by heat, delayed by cold, and are limited in their action only by the accumulated product of such action." - WALLER.

Ptyalin is a type of a group of bodies called Unorganized Ferments, or Enzymes. These ferments are the agents that produce the peculiar chemical changes that are the chief part of digestion.

\section{EXPERIMENTS WITH THE DIGESTIVE LIQUIDS:}

Label all the test tubes used in these experiments.

The action of the Saliva on starch.

1. Make a starch paste as follows: Place one gram of starch in a mortar, and rub up well with a little cold water. Add two hundred c. c. of boiling water, and boil for some time, stirring well.

2. Prepare a solution of grape sugar by crushing a few raisins and soaking them in water. To half a test tubeful of this solution add three or four drops of solution of caustic potash and two or three drops of a dilute solution of copper sulphate (blue vitriol); or, instead of using the caustic potash and copper sulphate, use "Fehling's test tablets," which can be bought of the druggist. Shake the liquid and boil. An orange-red precipitate indicates the presence of grape sugar.

3. Collect a teaspoonful of saliva in each of five test tubes; add water till the tubes are half full. Label the tubes A, B, C, D and E. Boil the liquid in test tube A.

4. Add a few drops of the starch paste to each of the 
test tubes. Set $D$ and $E$ in ice-water. To $B$ add a few drops of hydrochloric acid. Set $A, B$, and $C$ in water kept at $100^{\circ} \mathrm{F}$. After ten minutes, test $A, B, C$, and $D$ as in Exp. 2. In $A$ boiling destroyed the power of the saliva. In $B$ the acid prevents action as in the stomach. $C$ shows grape sugar. $D$ shows no change.

5. Transfer $E$ to warm water for ten minutes, then test to show that cold has not killed the saliva, but merely arrested its action. in $C$.

6. 'Test the action of saliva on raw starch, conditions as

7. Test both the saliva and the starch separately, to show that there is no sugar in them.

The action of the Gastric Juice.

Buy some pepsin of the druggist. Boil an egg hard, and thoroughly mince part of the white, best by rubbing it through a sieve. Add pepsin to a little of the egg in each of four test tubes half full of water. Label $A, B, C$, and $D$. To $B$ add bicarbonate of soda, to $C$ and $D$ a few drops of dilute hydrochloric acid. Set $A, B$, and $C$ in warm water, and $D$ in ice-water. Shake the tubes frequently.

Repeat $C$ with large pieces of egg to see the effects of imperfect mastication.

Try also pepsin that has been boiled.

Pepsin may be obtained by dissecting off the mucous coat of a pig's stomach, mincing, and soaking it in glycerin for a day or two, and straining.

Action of the Pancreatic Juice.

The pancreatic juice may be extracted from a fresh pancreas (taken from a pig) by mincing the pancreas, and soaking it in water for a few hours. But it will probably be more satisfactory to buy from the druggist preparations of Pancreatin and Amylopsin. 


\section{Emulsifying effects of Pancreatic Juice.}

Chop a pig's pancreas fine, and just cover with water. After two or three hours strain off the water. Take half a test tubeful of this extract, and add half its volume of oliveoil. Shake them thoroughly together. This will form a creamy mixture, an Emulsion.

Shake oil and water together in a test tube. They soon separate. Now add a little white of egg and shake again. A more permanent emulsion is formed.

2. The action of the Pancreatic Juice on starch.

Prepare starch paste as before. Add amylopsin now instead of saliva, and keep in a warm place, about the temperature of the blood or somewhat warmer.

Add hydrochloric acid and sodium bicarbonate as in the above experiment.

Test the result as in the case of saliva. It will be easier to use the amylopsin than to prepare the extract of pancreas for one's own use, as there are excellent preparations in the market.

3. The action of the Pancreatic Juice on Proteids.

Take. some hard-boiled white of egg and chop fine, or rub through a sieve. Put some of this into two test tubes; half fill with water; add pancreatin. To one add a little dilute hydrochloric acid, and to the other a little sodium bicarbonate. Set both in a warm place and shake frequently. The effect of temperature may be tested as before. If pancreatin is not at hand, make a glycerin extract of pancreas as follows : "Obtain a fresh pig's pancreas. Lay aside in a cool place twenty-four hours. Mince, and extract for two days in twice its bulk of glycerin. Strain off the glycerin extract. Dilute the glycerin extract with ten times its bulk of water."

Put into this some hard-boiled white of egg, and keep in a warm place. To one test tube add a little dilute hydro- 
chloric acid, and add to another some bicarbonate of sodium. Find also the effect of first boiling the pancreatic preparation.

In these experiments we find that saliva turns starch to grape sugar, if not in an acid solution and if at the proper temperature. That pepsin dissolves proteids in an acid (hydrochloric) at the right temperature. The proteid is turned to peptone, and becomes soluble and diffusible, capable of absorption through the walls of the stomach and intestine.

We find that the different elements of the pancreatic juice can, in alkaline solution, and at the right temperature, emulsify fats, turn proteid to peptone, and convert starch into grape sugar.

The Bad Effects of Imperfect Mastication. - If we swallow food before it is thoroughly ground and mixed with the saliva, the stomach and other parts of the digestive organs will require much more time to reduce the food to a liquid form. Further, when eating hastily, we are very apt to eat too much. Thus we may give the stomach a double amount of material to handle, and the material may not be half so well prepared as it should be. The work thus thrown upon the stomach may easily be made fourfold. Of course the organs suffer; and sooner or later, if this treatment is continued, they must break down.

Not only mastication, but the whole process of digestion, goes on better when the body and mind are at rest and in a peaceful and contented condition, as not only the salivary glands, but all the glands, are under the control of the nervous system, and are greatly influenced by the condition of the body. During a meal, and for a short time before and after, all thoughts of one's occupation, and especially all anxiety, should be absolutely dismissed from the mind. For those whose digestion is not strong, it is especially desirable to secure a period of rest after each meal, taking a lounge or 
easy-chair, closing the eyes, and, as nearly as possible, closing the mind; for some, even a short nap is very helpful.

During a meal there should be conversation on topies of general interest. "Chatted food is half digested."

It is said that the people of the United States are nervous, and eat, as they do nearly everything, hastily. Deliberation in eating adds to dignity as well as health, and properly may be considered an evidence of culture.

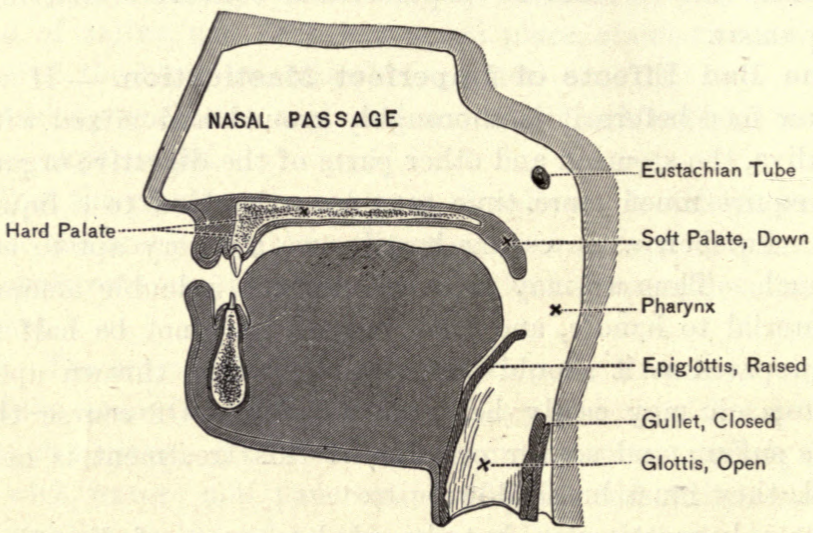

Fig. 59a. Diagram, Showing the Positions of the Organs of the Mouth and Throat During Breathing.

The Process of Swallowing. - The cavity back of the mouth, beyond the soft palate, is the Pharynx. The pharynx is a funnel-shaped cavity, communicating above with the passages from the nostrils; in front it opens into the mouth; below it connects with the windpipe, through the glottis, and with the gullet, which, as we have seen, lies just back of the windpipe. 
When the morsel of food is ready to be swallowed, the tongue pushes it back into the pharynx; the soft palate is raised to shut off the passage into the nasal cavity; the larynx is pulled upward and forward; the epiglottis is pulled down over the glottis, or opening of the windpipe; and the base of the tongue extends back over the epiglottis; thus the air passages, above and below, are shut off, and the food passes over the epiglottis into the gullet. The muscles of the pharynx

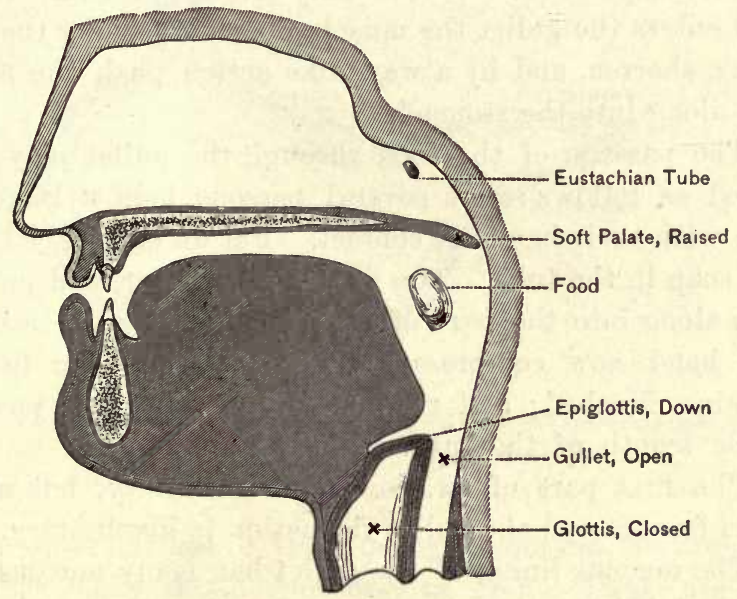

Fig. 59b. Diagram, Showing the Positions of the Organs of the Mouth and Throat During Swallowing.

also do their part in pushing the food along. As soon as the food has passed over the epiglottis, the epiglottis rises to its upright position, and the soft palate drops back to its place, leaving the air passages again open. As we are swallowing only a small part of the time, the passageway naturally stands open to the air; and when we swallow, the parts are, by muscular effort, temporarily adjusted for this work. It is to be 
observed that the food tube and the air tube cross, and that the pharynx is their crossing. There is a spring switch (to borrow a term from the railway) which keeps the track open for the air, which is all the time passing; but when the food comes along, the switch must be held open until it has passed.

As we saw when we examined the gullet, it has an outer muscular coat, and an inner mucous coat. The muscular coat has two layers, an inner with circularly arranged fibers, and an outer layer with longitudinally arranged fibers. When the food enters the gullet the muscle fibers, especially the circular fibers, shorten, and by a wave-like action push the mass rapidly along into the stomach.

The passage of the food through the gullet may be illustrated as follows: Let several persons hold a large rubber tube with their hands in contact. Put an egg-shaped piece of wet soap in the tube. The first hand is shut, and pushes the soap along into the part of the tube held by the next hand; this hand now compresses the tube, while the first hand remains clinched; and so, in turn, the object is pushed the whole length of the tube.

The first part of swallowing is voluntary, but after the bolus has entered the gullet the action is involuntary.

The mucous lining of the gullet has many mucous glands, which lubricate the passageway by the mucus which they secrete.

The Stomach. - Just beyond the diaphragm the digestive tube widens suddenly, forming the stomach; the stomach is an oval sac lying just beneath the diaphragm, with the large end to the left and the small end to the right. The smaller end, by narrowing, becomes the small intestine. When the stomach is empty it collapses, as its walls are soft and flexible. When distended it may hold three pints, or even more when abnormally distended. 
The muscular coat of the stomach consists of three layers, distinguished by the arrangement of the fibers, a circular layer, a longitudinal layer, and an oblique layer.

The mucous lining is somewhat loosely attached to the muscular coat, and when the stomach collapses the mucous coat is thrown into folds, usually running lengthwise.

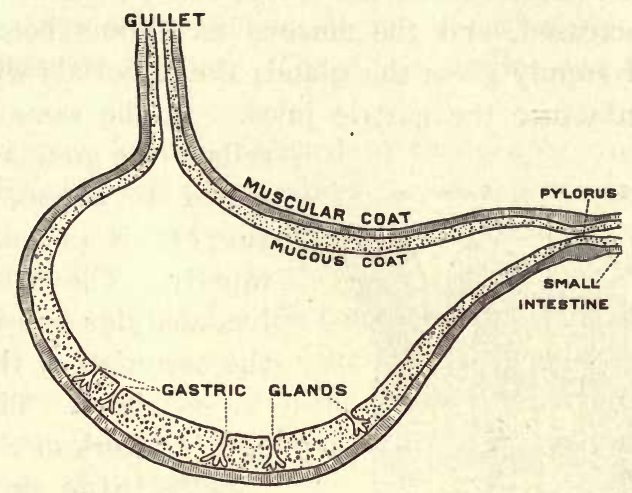

Fig. 60. Longitudinal Section of Stomach, Showing Gastric Glands in Positlon. (Dorsal View. Mucous Coat Unduly Thickened.)

If the inner surface of the mucous membrane is examined with a lens many small holes may be seen. These are the mouths of the ducts of the Gastric Glands. If the duct is traced inward, it is found to divide into several branches, usually two or three. These gastric glands vary somewhat in their structure in different parts of the stomach. The liquid secreted by the different glands also varies considerably, but the liquid as a whole is called the Gastric Juice. The gastric juice is chiefly water, containing a ferment, or enzyme, called Pepsin, and a small amount of acid. The amount of gastric juice secreted daily has been estimated at from five to ten 
quarts. Of course, we must bear in mind that nearly all of this is again absorbed from the digestive tube, and is not a permanent loss to the body.

The mucous membrane is abundantly supplied with blood tubes; but during the time of its rest the blood flow here is diminished, and the membrane is comparatively pale. But as soon as food is introduced into the stomach, the blood flow is greatly increased, and the mucous membrane becomes red. This blood supply gives the glands the materials with which they manufacture the gastric juice. At the same time the cells of the glands are stim-

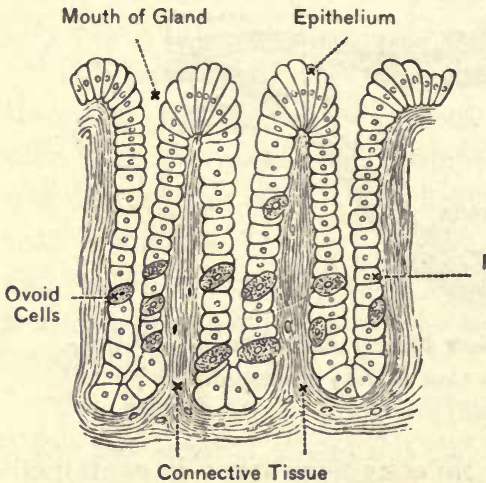

Fig. 61. Three Glands of the StomachCardiac Part. ulated to action, and the secretion is poured out rapidly. The alkaline saliva also aids in stimulating the secretion of the gastric juice. The special Principal, work of the gastric
Cells juice is accomplished by the pepsin, aided by the acid; these convert proteids into a soluble substance, called Peptone, which can be absorbed through the walls of the digestive tube into the blood. Rennet, used in cheese-making, is a familiar substance obtained from the fourth stomach of the calf. Now, when milk enters the human stomach it is curdled; that is, the casein previously dissolved in the liquid milk is coagulated. This curdling, or coagulation, is attributed to a ferment in the gastric juice called Rennin; and it seems to be entirely distinct from pepsin. At the same time all the food is soaked by the gastric juice, the process being greatly 
assisted by the churning motion of the stomach caused by the action of the muscular coat. The food is thus gradually reduced to a pulpy mass called Chyme. During the first part of digestion in the stomach, the thick ring of circular fibers called the Pylorus (gatekeeper) around the opening of the stomach into the intestine, keeps the passage nearly closed, leaving a small orifice for liquids only.

Such rings of muscular fibers, guarding openings, are called Sphincter Muscles. There is a similar one at the anal opening.

But as the food is reduced to the proper condition the pyloric muscles relax, and allow the chyme to pass into the intestine. And at last any indigestible substances are allowed to pass (usually).

The time required for the complete digestion of any ordinary meal is from three to four hours; though this may be much longer if very indigestible substances have been eaten, or if the condition of the body or mind is such as to retard the process of digestion.

Some parts of the food that are already digested, or such matters as are soluble, e.g., water containing sugar, peptone, salts, etc., may be absorbed immediately through the walls of the mouth and stomach into the blood capillaries.

Recent experiments show that the amount of absorption from the stomach is much less than was formerly supposed; water, for instance, "when taken alone, is practically not absorbed at all in the stomach. As soon as water is introduced into the stomach, it begins to pass out into the intestine, be. ing forced out in a series of spurts by the contractions of the stomach."

The rest of the food, now called chyme, is passed on into the small intestine. It is acid, and in a liquid or semiliquid condition. Chyme, as it enters the intestine, is a mixture of 
digested, partly digested, and undigested materials. Some of the starch has been changed to sugar, but only a small part, owing to the short time of mastication. (It is believed that the slower mastication in the herbivora changes much more of the starch to sugar.) The bulk of the starch is unchanged. Some of the proteid is already changed to peptone. Part is still proteid, while part is in an intermediate stage between proteid and peptone. Fat is essentially unchanged, but is melted by the heat of the mouth and stomach, and is more or less divided into small drops by mastication and the movements of the stomach. For instance, in eating bread and butter, the melting butter will be finely mixed with the bread as it is chewed. The water in the chyme was partly taken as such, and partly derived from the saliva and gastric juice. There are also present ptyalin, pepsin, mucus, salts, and some indigestible substances. At intervals the sphincter muscles of the pylorus relax, and the con-

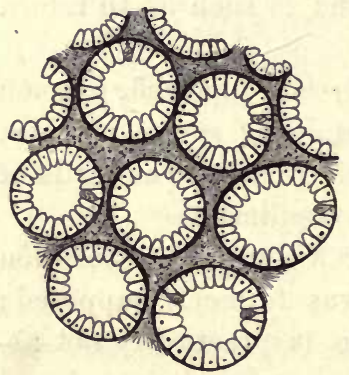

Fig. 62. Horizontal Section through the Mucous Membrane of the Intestine, showing Intestinal Glands in Transuerse Section. (Highly Magnified.) tractions of the stomach send the liquid mixture into the intestines by spurts.

The Intestine. - The small intestine has essentially the same structure as the parts of the digestive tube already studied, namely, a mucous lining beset with an immense number of tubular glands, called Intestinal Glands. These secrete a liquid collectively called the Intestinal Juice, whose exact work is not well known, but which may be said to complete the work of the other secretions. The intestines have also the muscular coats, about as described. And the muscular coat has 
the same work of mixing the juices with the food, and of moving it along.

Soon after the chyme enters the small intestine it has poured upon it two liquids, which enter the intestine in one common stream; these are the Bile and the Pancreatic Juice. Two large compound glands, the Liver and Pancreas, lie close to the stomach; their ducts join before they enter the intes-

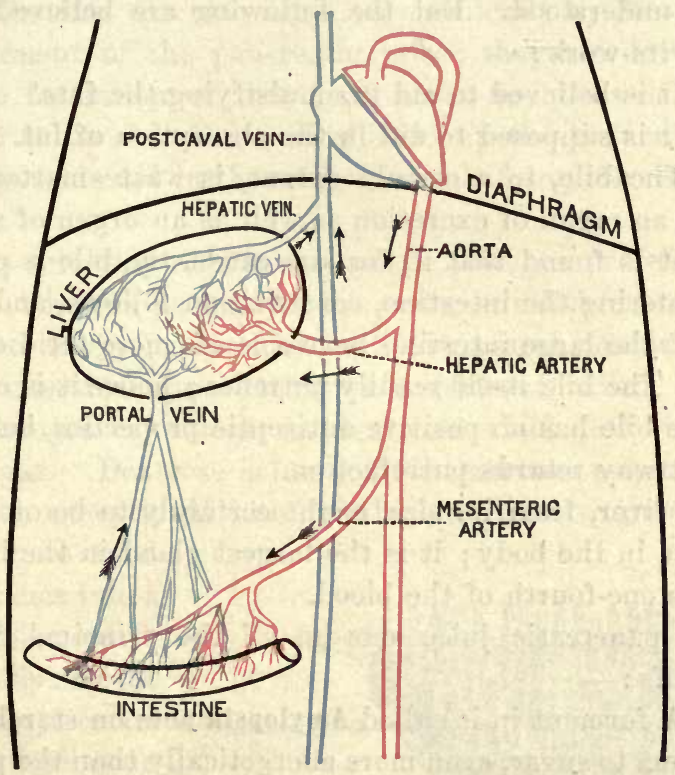

Fig. 63. Diagram of Portal Circulation.

tine into which these juices are emptied a few inches beyond the stomach.

The liver, like the pancreas, receives a supply of blood from the aorta; but the liver is peculiar in receiving, through 
the portal vein, nearly all of the material obtained by absorption from the digestive tube. The blood from the portal vein is distributed through the liver. And from this blood the liver manufactures at least two important substances, the bile and the liver-starch, or Glycogen.

The bile is secreted all the time, but more actively during digestion. The part made while digestion is not going on is stored in the bile-sac. The functions of the bile are still poorly understood. But the following are believed to be a part of its work :-

1. It is believed to aid in emulsifying the fats.

2. It is supposed to aid in the absorption of fat.

3. The bile, to a certain extent, is waste matter; so the liver is an organ of excretion as well as an organ of secretion.

4. It is found that if, for any cause, the bile is prevented from entering the intestine, constipation follows, and the contents of the large intestine have a much more fetid odor than usual. The bile itself readily putrefies; hence it is concluded that the bile has no positive antiseptic properties, but in some indirect way retards putrefaction.

The liver, from its size, ought certainly to be of great importance in the body; it is the largest gland in the body, and receives one-fourth of the blood.

The pancreatic juice acts on all the principal classes of foodstuffs :-

1. A ferment in it called Amylopsin acts on starches, changing them to sugar, even more energetically than the ptyalin of the saliva.

2. Another constituent of pancreatic juice is Trypsin ; like the pepsin of gastric juice, this ferment has the power of cnanging proteids to peptones.

3. The pancreatic juice also acts on the fats, in two ways:(a) It emulsifies them, i.e., the fat is divided into exceed- 
ingly fine drops, each enreloped in a coating of albuminous substance. Milk is a natural emulsion. Examine a drop of milk under the microscope. An emulsion can be made artifcially by shaking together water, oil, and white of egg. The shaking breaks the oil into fine drops, which would soon gather again if no other substance were present; but it is supposed that the albumen forms a thin coating around each droplet, enabling it to remain distinct in the liquid.

(b) The fats are also acted on chemically by Steapsin, another ferment of the pancreatic juice; they are decomposed with the formation of free fatty acids, and thus more fully prepared to be absorbed and to build up the tissues. These free fatty acids aid in the work of emulsifying the rest of the fat.

Saliva acts only on starch ; gastric juice, on proteids ; bile, on fats; whereas pancreatic juice acts on all three, and probably more energetically than the above-named liquids.

The intestinal juice contains a ferment called Invertin, which changes cane sugar to dextrose, which is a variety of grape sugar. Dextrose is less soluble than cane sugar. Here, then, is an exceptional case, in which a digestive process converts a more soluble substance into a less soluble form.

The bile and the pancreatic juice are alkaline, and overcome the acidity of the chyme.

Absorption. -

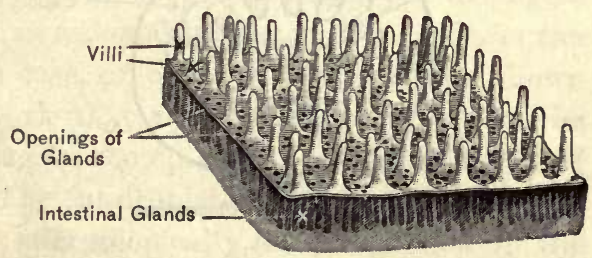

Fig. 64. Mucous Membrane of Small Intestine. The mucous membrane of the small intestine is thrown into ridges; but, unlike those of the stomach, they run transversely. 
Again, while the folds in the lining of the stomach are temporary, these are permanent. They serve to increase the surface of the lining, and to retard the passage of the food material, and so to aid the process of digestion and of absorp. tion.

Further, the surface of the mucous membrane is thickly beset with little cylindrical projections, like the "pile" on velvet. These pro-

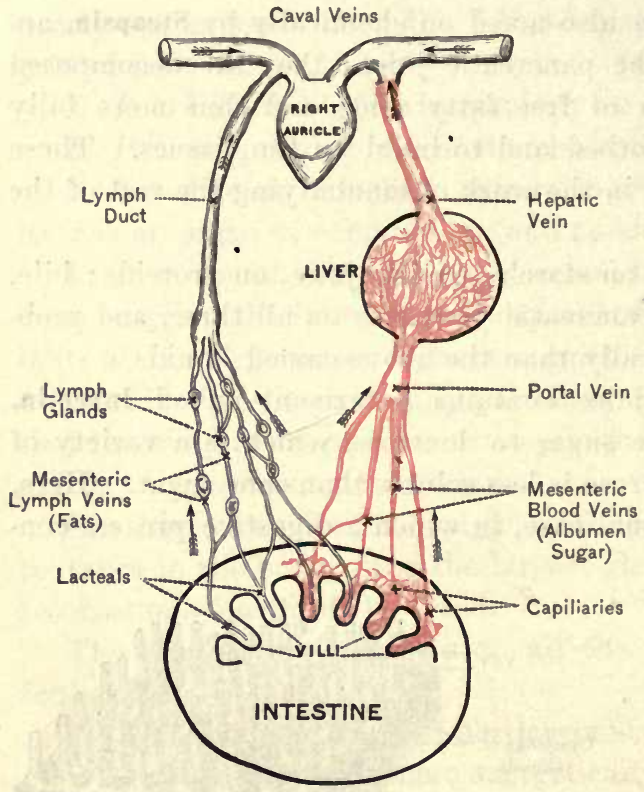

Fig. 65. Plan of Absorption. jections are called Villi (singular, villus). The villi greatly increase the absorbing surface of the small intestine. In each villus is a network of Blood Capil. laries, and the beginning of lymphatic capillaries called Lacteals.

In the villi the largest part of the work of absorption is done. The fats are absorbed by the lymph capillaries, and the rest of the foods by the blood capillaries. It should be carefully noted that nearly all of the foods but the fats go at once to the liver, through the portal vein; but the fats are carried by the main lymph duct (the Thoracic Duct) 
to be emptied into the subclavian vein in the neck, hence do not directly pass through the liver.

If a solution of salt and one of sugar are brought into contact, they will gradually mix ; and this is called Diffusion. If, now, a piece of parchment be tightly tied over the end of a tube, and one of the above solutions be placed inside, and the other around the tube, they will still diffuse through the membrane, and mingle. This is called Osmosis, or Dialysis ; and the parchment is called a dialyzing membrane. In the digestive tube the mucous membrane represents the dialyzing membrane, with blood or lymph on one side, and the contents of the digestive tube on the other. Soluble materials, such as peptones, sugars, etc., pass through the mucous membrane into the blood.

"The process of osmosis, and to a lesser extent of filtration and imbibition, as they are known to occur outside the body, were supposed to account for the absorption of all the soluble products. This belief has now given way, in large part, to newer views, according to which the living epithelial cells take an active part in absorption, acting under laws peculiar to them as living substances, and different from the laws of diffusion, filtration, etc., established for dead membranes.

"Unlike sugars and peptones, fats are absorbed chiefly in a solid form - that is, in an emulsified condition. There can be no question, in this case, of osmosis. It has been shown by nearly all recent work that the immediate agents in the absorption of fats are again the epithelial cells of the villi of the small intestine. The fat-droplets are taken up by these cells, and can be seen microscopically after digestion in the act of passing, or rather of being passed, through the cellsubstance. The epithelial cells, in other words, ingest the fat-particles lying against their free ends, and then pass them slowly through their cytoplasm into the substance of the villus." - Howels. 
The Lacteals and Lymphatics. - While the main work of the lymphatics, as we have seen, is the carrying of lymph from the tissues of the body generally to empty into the veins of the neck, the lymphatics of the intestines have another important function.

They absorb and carry the fatty portions of the digested

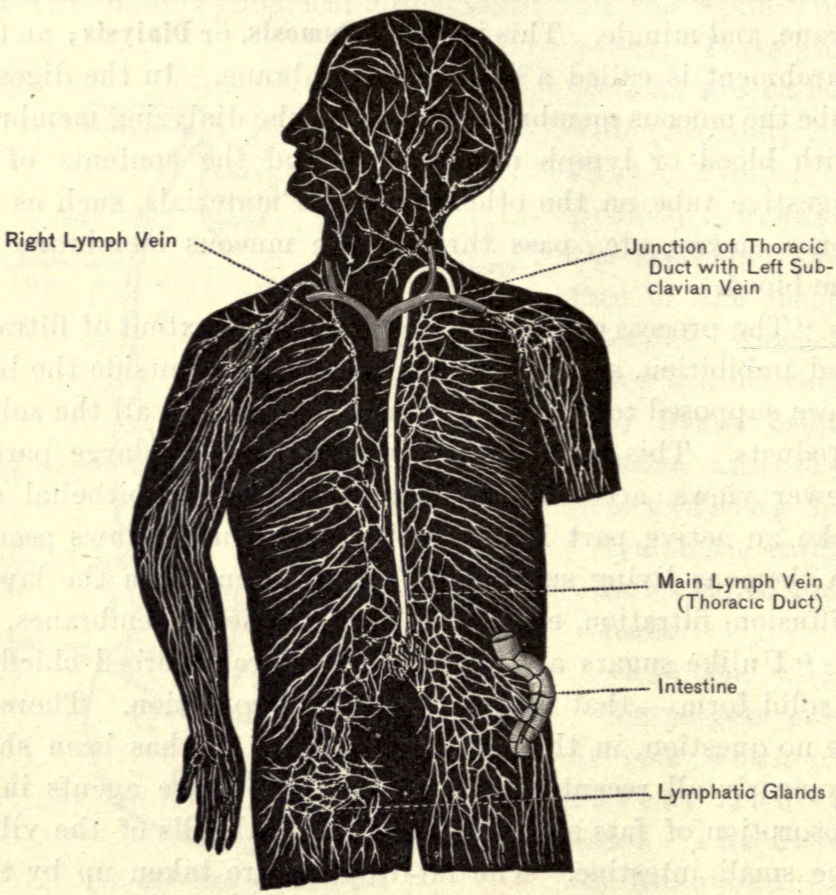

Fig. 66. Lymph Veins-Lymphatics.

food into the general circulation. During most of the time the thoracic duct and the lymphatics of the intestines would hardly be noticed, because they are filled with the clear lymph. 
But after digestion of fatty matter they are filled with the white Chyle, and are easily seen.

To show the thoracic duct, feed a kitten or puppy on rich milk, and after two or three hours kill it as directed on page 67. As soon as you are sure it is dead, open the abdominal cavity, and spread out the mesentery. The white lacteals, filled with chyle, will be seen radiating through the mesentery. Press on some of these, and it will be seen that they are thin tubes filled with a white liquid. They converge toward the place of attachment of the mesentery to the dorsal part of the abdomen. On the dorsal wall of the abdomen, just posterior to the diaphragm, the Receptacle of the Chyle, or the beginning of the main lymph vein (thoracic duct), should be found. Trace it anteriorly through the chest alongside the aorta to

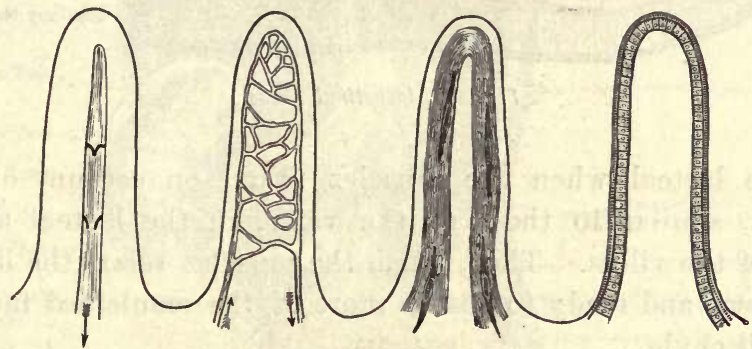

Lacteal with Valves

Capillaries

Muscles

Epithelium

Fig. 67. Elements Entering into the Structure of a Villus.

its mouth, near the junction of the left subclavian and jugular veins.

The thoracic duct may be injected, though it is not always easy to do so. Use a small syringe and the starch preparation described on page 66. After finding the receptacle of the chyle, snip a small hole in its posterior end, and quickly 
insert the tip of the syringe nozzle, and inject. It will not be necessary to ligature, for there are so many valves in the duct that if a fair amount of the starch preparation be introduced there will be little reflow. For this the yellow-colored mass may be used.

In each villus there are plain muscle fibers. When these shorten they squeeze the chyle, that has already been absorbed, into the lymph tubes of the wall of the intestines, and on into the main lymph duct. The chyle cannot return

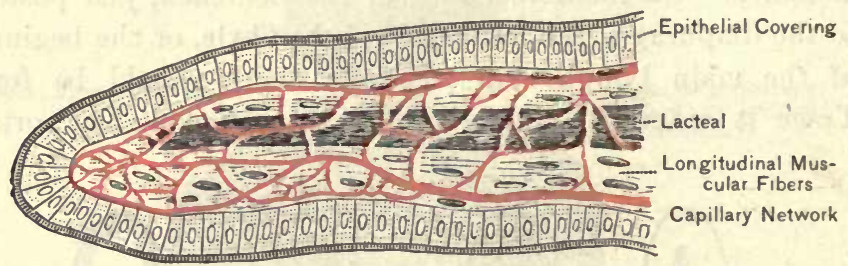

Fig. 68. Intestinal villus.

to the lacteal when the muscles relax, on account of the valves, similar to those of the veins, in the lacteal at the base of the villus. Then, when the muscles relax, the lacteal is empty, and ready to absorb more of the emulsified fat that we call chyle.

The whole digestive tube may be briefly and roughly described as a muscular tube of varying diameter, lined by mucous membrane. The muscular coat propels the contents and mixes them with liquids; the mucous. coat is beset with glands, making liquids, some of which merely soak the food, others act on it chemically, while mucus serves to lubricate the surface. It seems that these myriads of Simple Glands are not enough, so several large Compound Glands lie alongside the food tube, and empty their secretions into it by Ducts; 
these supplementary glands are the salivary glands, the pancreas, and the liver.

The length of the small intestine is about twenty-five feet;

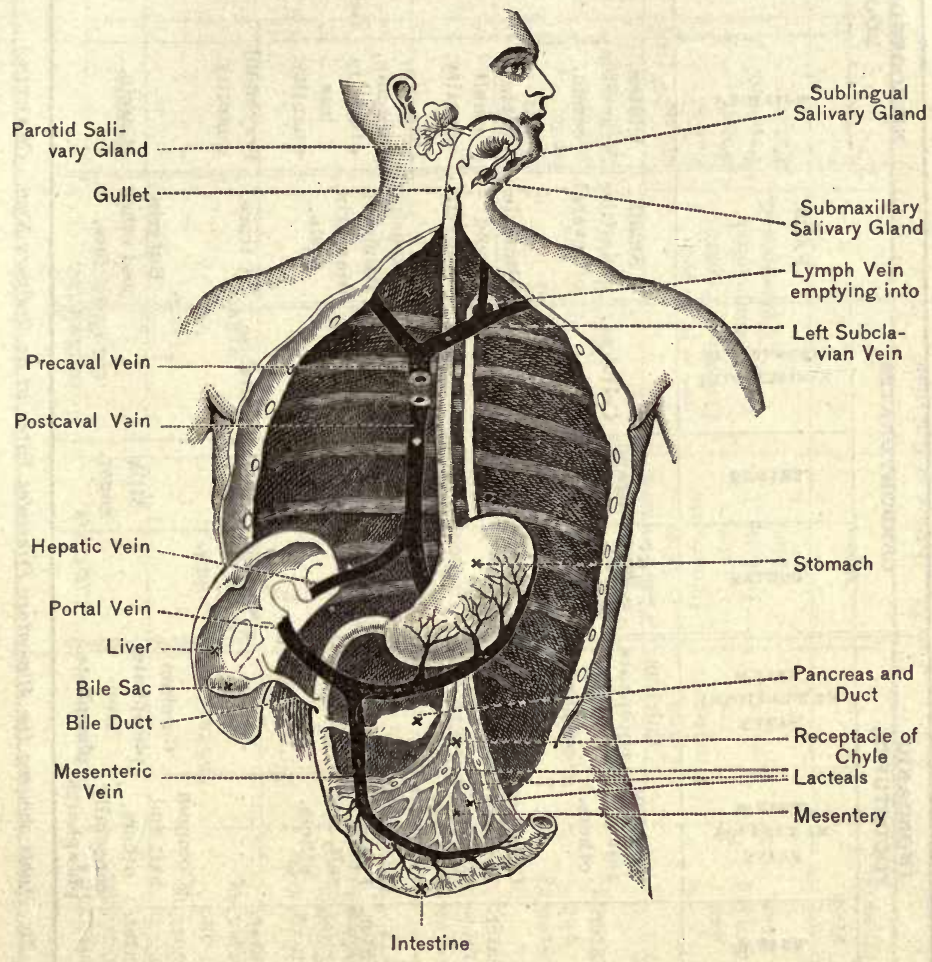

Fig. 69. Diagram of the Organs Concerned in the Conversion of Food into Blood.

and of the large intestine five or six feet. As in the lower mammals, the large intestine is not a direct continuation of the small; that is, the small intestine opens into the large near the beginning of the latter, so that there is a short blind 


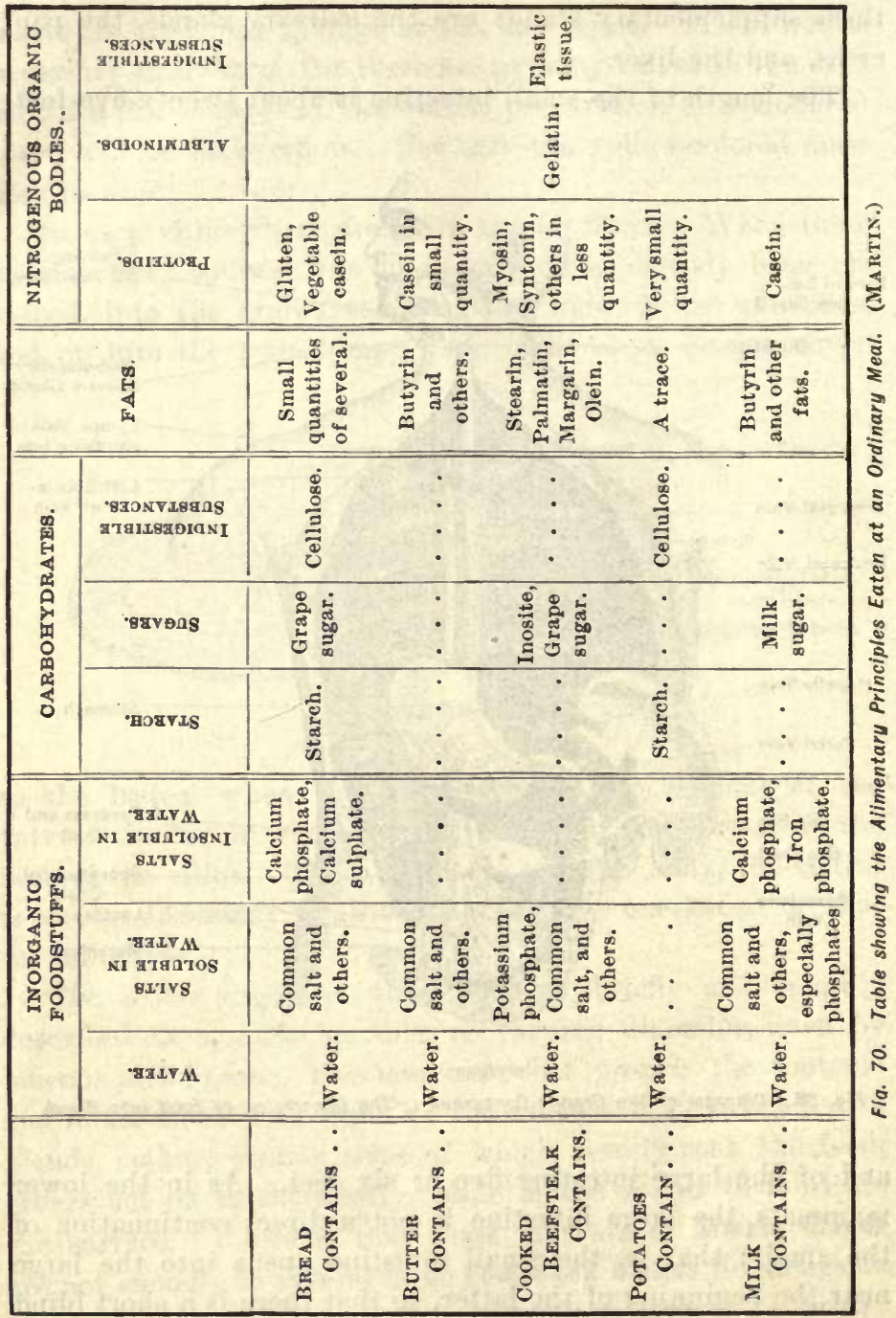




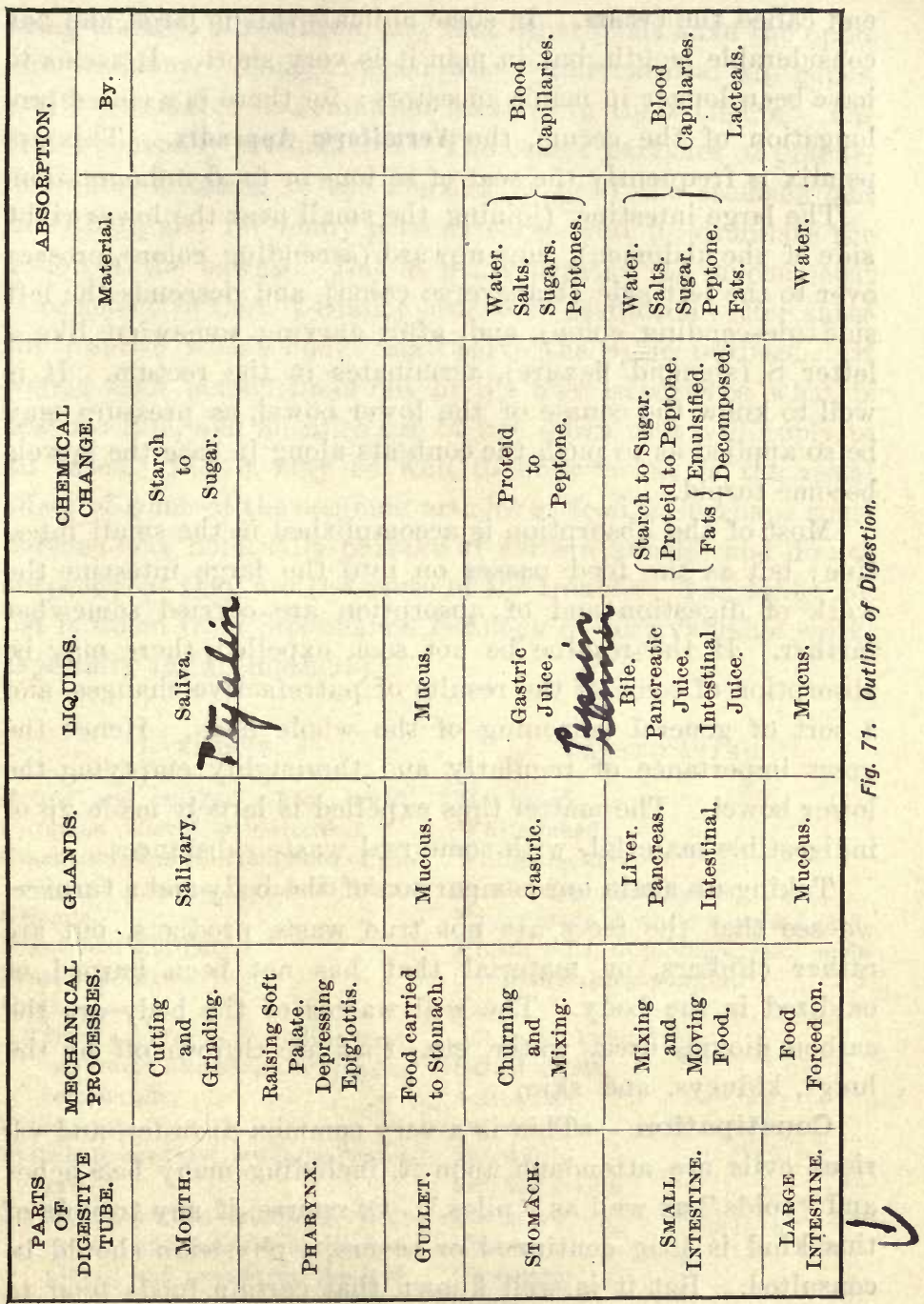


end called the Cecum. In some animals this is large, and has considerable length, but in man it is very short. It seems to have been longer in man's ancestors; for there is a closed prolongation of the cecum, the Vermiform Appendix. This appendix is frequently the seat of serious or fatal inflammation.

The large intestine, (joining the small near the lower right side of the abdomen), runs upward (ascending colon), crosses over to the left side (transverse colon), and descends the left side (descending colon), and, after curving somewhat like a letter $\mathrm{S}$ (sigmoid flexure), terminates in the rectum. It is well to know the course of the lower bowel, as pressure may be so applied as to push the contents along in case the bowels become torpid.

Most of the absorption is accomplished in the small intestine; but as the food passes on into the large intestine the work of digestion and of absorption are carried somewhat farther. If the residue be not soon expelled, there may be absorption of some of the results of putrefactive changes, and a sort of general poisoning of the whole body. Hence the great importance of regularly and thoroughly emptying the lower bowel. The matter thus expelled is largely made up of indigestible material, with some real waste substances.

Taking up again our comparison of the body and a furnace, we see that the feces are not true waste products, but are rather clinkers, or material that has not been burned or oxidized in the body. The real wastes of the body are the carbon dioxid, urea, water, etc., that are thrown off by the lungs, kidneys, and skin.

Constipation. - This is a very common disorder, and various evils are attendant upon it, including many headaches and "colds" as well as "piles." Of course, if any trouble of this kind is long continued or severe, a physician should be consulted. But it is well known that certain foods tend to 
bring on such a condition, and that other foods have the opposite tendency. Thus, cracked wheat and oatmeal are genererally considered as somewhat laxative in their effects. The fruits generally are laxative. The coarse particles of graham flour are irritating to the mucous lining of the stomach and intestines, and for many persons serve well to stimulate the action of the bowels. But in many persons the mucous coat is so sensitive that it cannot bear such irritation. For these the "entire wheat flour" may serve the same purpose. Of course each person finds out by his own experience what is best for him, and no rules can be laid down that will apply to all cases. But it may be well to know what is the usual effect of some of the common articles of food, as perhaps some persons may habitually partake of certain articles and do not suspect that they are the cause of the trouble. The following list is taken from Stockham's Tokology (a very valuable work, especially for all mothers).

\section{LAXATIVE.}

Rolled and cracked wheat bread, gems, biscuit, griddlecakes.

Crackers and mush from flour of the entire wheat and graham flour.

Granula.

Bran gruel and jelly.

Fruit puddings.

Fruit pies.

All fresh acid fruits, including tropical fruits, like bananas, oranges, lemons, etc.

Dried fruits.

French prunes and prunellas, eaten raw.

Stewed dried fruits containing hydrocyanic acid, of which peaches, plums, and prunes are the best.

Now Orleans molasses.

\section{CONSTIPATING.}

Hot bread.

White bread.

White crackers.

Black pepper and spices.

Pastry made of white flour and lard.

Bread, rolls, dumplings, etc., made with baking-powder:

Cake.

All custard puddings.

Salted meats.

Salted fish.

Dried meats.

Dried fish.

Smoked meats.

Poultry.

Cheese.

Chocolate.

Cocoa. 


\section{LAXATIVE.}

Rhubarb.

Onions.

Celery.

Tomatoes.

Cabbage, raw.

Coru.

Squash.

Cauliflower.

Green peas.

Spinach.

Beets, etc.

Liver.

Oysters.

Wild game.
CONSTIPATING.

Boiled milk.

Tea.

Coffee.

Coffee made of wheat, corn, barley, toast, etc.

Beans (dried).

Potatoes.

Farina.

Sago.

Starch.

Tapioca.

Rice.

Raspberries.

Blackberries.

Hygiene of Digestion. - A prime requisite for a good digestion is a tranquil condition of the whole body, especially of the nervous system. We see that the blood must be massed in the digestive organs at the time of digestion. As there is a limited amount of blood in the body, it is evident that if more is sent to one part, other parts must at the time receive less. If we try to study hard immediately after eating, we are calling the blood away from the organs of digestion, and to that extent interfering with the process of digestion. If we exercise the muscles too vigorously soon after eating, we call the blood to the muscles, and so call the blood away from the stomach and intestines. If, after prolonged study, one is unable to obtain sleep, it may sometimes be efficacious and very desirable to eat a little of some very simple food for the purpose of drawing off the blood to the stomach, and thus relieving the brain. A little muscular exercise may accomplish the same result, or a footbath may be employed. For many persons it would probably be better to take a simple lunch than to go to bed hungry, although one should be careful not to abuse the stomach. 
It is exceedingly difficult to lay down general rules in regard to diet. To a certain extent each person must be a law unto himself, for what agrees well with one may act almost as a poison to another. Moderation should always be observed, especially in taking foods to which we are not accustomed.

A Good Dinner. - Suppose one were to sit down to eat dinner when ravenously hungry. If in such a conditicn one begins with solid food, he is likely to eat too fast. Hunger is a demand of the system for food. It takes some time for solid food to go through all the processes of digestion, and be absorbed into the system and appease hunger. But if a soup be first taken, which is readily absorbed, the demand of the system will begin to be met, and there will not be the same tendency to rapid eating. Further, a warm soup stimulates the blood flow in the mucous membrane, and thus prepares for more thorough digestion. It is more easy after a soup to deliberately masticate the solid portion of a meal. Dessert and sweetmeats, following a meal, are often very helpful by further stimulating the secretion of the glands. Nuts, which are not very digestible, are beneficial if eaten sparingly. The agreeable taste stimulates the salivary glands, and the alkalinity of the saliva stimulates the gastric glands to increased activity. The same may be said of cheese.

\section{"Cheese is a surly elf,}

Digesting all things but itself."

The average pie needs some extra help for its digestion. Donoghue, formerly champion long-distance skater, when asked if he dieted in preparation for a race, said he avoided pastry. If the vigorous digestion of a man skating for hours daily in zero weather eannot profitably manage pie, how in the case of sedentary persons? If pie is eaten, it should be masticated with very great thoroughness. 
Hot drink, with a meal, whether it be tea, coffee, or merely hot water, is usually beneficial; especially to a weak digestion, when taken before meals.

Undoubtedly most persons would be better off if they did not eat puddings and pastries. Fruit is best taken before meals, especially before breakfast.

"Taking Cold." - So long as one is actively exercising, he is not likely to take cold. But if one rests in a cool place, especially when he is warm, he is, as we all too well know, likely to take cold. As we saw when we were studying the circulation of the blood, the application of cold to the skin causes the arteries (through reflex action) to become smaller. Thus when resting in a cool place the skin becomes pale and cold.

During a "cold" there is fever. The regulation of the heat by the skin is interfered with. At the same time it is often noticeable that the urine is more abundant than usual. As cold may lead to fatal lung disease, so it may be the beginning of some disease of the kidneys that may, in the end, bring fatal results.

Diarrhea, which is a catarrhal condition of the intestine, may follow, or be associated with, a cold; and as a result of this the process of absorption is often largely checked. There is a great increase in the secretion of mucus by the mucous glands in the intestinal wall. As the various liquids of digestion are all taken from the blood, it is evident that if some returns are not soon made, the system must become bankrupt. It is, then, more easy to understand the excessive weakness and feeling of utter prostration that we experience during an acute attack of diarrhea. We can now understand where all the material comes from to make the profuse discharges, especially after we have ceased eating for some time.

It is a significant fact that diarrhea is usually called "sum- 
mer complaint." During the warm summer nights we are tempted to go to sleep with very little covering over our bodies. But it almost always grows cool before morning. The common summer diarrhea is, in many cases, due to bacteria taken in food; but, on the other hand, may be simply a "cold in the bowels."

Bathing. - One purpose of bathing is to cleanse the skin. For this purpose warm water is best; and it is desirable to use soap, especially on those parts which are especially exposed to contamination, such as the hands and feet, the armpits and groins.

Another important function of bathing is to act as a systemic tonic. For this purpose cold bathing is better; but this should not be too long continued, and must be followed by brisk friction to give the skin a ruddy glow. For this kind of bath a tub is not necessary, and hardly desirable. The water may be quickly applied by means of a sponge, and the body thoroughly rubbed with a coarse towel. The whole process should be completed very quickly, especially if the room be not warm.

Instead of the sponge and the ordinary form of towel, it may be found more convenient to use bath mits made of Turkish toweling. These are easily made, and are somewhat more convenient, as thus frietion may be more readily applied than with a towel; which is apt to slip in the hand. The two hands may be used at the same time, and the whole time of the bath need not exceed two or three minutes. At the beginning of a bath, cold water should be applied to the head and face.

For students, or others who do not take a great deal of vigorous exercise, which keeps the skin active, this means of keeping the skin active is especially valuable. The use of warm water for cleansing seems best adapted (for busy people) 
to the time of going to bed. But the best time for the cool bath is on getting up in the morning.

Prolonged warm baths are debilitating, and probably increase a tendency to take cold, whereas cold bathing is one of the very best means of fortifying against cold, and especially against the tendency to take cold on slight exposure. For most persons a cool sponge bath, on rising, will act as a most excellent tonic; but if it seems to produce neuralgia, it should be used with caution.

We have learned that the blood supply to any organ is regulated by the action of the plain muscle fibers in the walls of the small arteries. Now, when we are subject to changes in temperature these muscles get exercise, and one writer has well called the cold bath the "gymnastics of the plain muscle fibers," and we can understand how the system can be trained to adjust itself to cold, and enabled to avoid "taking cold" so frequently.

There are undoubtedly many persons who do not profit by cold bathing, but probably many of these would soon adapt themselves to it by beginning with tepid water and gradually using cooler. To stand stripped in a cold room, of course, is not a safe thing to do. And the great secret of the benefit that may be expected from the operation, as most people are situatcd, is to be very brisk, the whole process occupying only a few minutes. Many are opposed to cold sponge bathing, and condemn it without reserve, when, probably, they have never really given it a fair trial.

Let it be repeated, with emphasis, that for students it is one of the very best means of preserving health.

Exercise. - The full significance of the benefits of muscular exercise could not be understood when we studied the muscles, and before we had studied the blood and its work in the tissues of the body generally. Now we can comprehend 
how exercise stimulates the cells to activity, renews the lymph around the cells both by quickening the blood flow and by pressure on the lymph tubes; how the glands of excretion are set to work more actively, and the more rapid blood stream brings away the material to be thrown out.

Exercise is not merely for the muscles. It quickens the action of the whole body by increasing cell activity. It helps to clean out the system and to clear the brain as well. We read Blaikie's admirable book, How to Get Strong, and learn not merely to strengthen the muscles, but how to get strong to do the work we have to do daily, how to feel well every day, how not only to do our work, but to do it gladly, and with a little extra good cheer that may radiate from us and inspire others. We have no right and no need always to carry the sour visage of a devitalized body. Good health is attainable, and ought to be attained, by nearly all. Attention must be paid to the laws of our being. It takes some effort, mental as well as physical, to adopt and observe regular hours for exercise and relaxation, and to be careful in diet.

But nature rewards for obedience by the delight of a healthy body; and she never forgets and never forgives, nor fails to punish every violation of every one of her laws. Nature makes no threats beforehand. She does not even tell us her rules. But we may find what they are by careful observation.

Many men would live longer, feel vastly better, and do greater good in the world, if they would take regular and systematic exercise or recreation (and this should be, literally, re-creation). It is a short-sighted policy to say, "I cannot afford the time," and mortgage one's future. The latter half of every person's life ought in many respects to be by far the most productive of good. But many cut off this half, or render it less productive, through breaking down in health as a 
consequence of trespassing on the laws of hygiene. Thus one defeats his own ends in life, and robs the world of the debt he owes it, that of returning to it, in his riper years, something for the help it gave to him in his early years while he had not yet reached the fullest mental maturity. It is sad enough that so magnificent a structure as the human body must perish and become part of the common clay. But it is infinitely more sad to think that it has not fulfilled its purpose when the end comes in what should be mid-career. Each of us should leave the world better than he found it, and our ability and opportunities for doing this increase as we reach middle life.

Forms of Exercise. - In selecting the kind of exercise the old lines fit well : -

"In whate'er you sweat, indulge your taste;

The toil you hate fatigues you soon,

And scarce improves your limbs."

Of course this does not mean that a boy should refuse to saw wood because he dislikes it, and spend all his time playing ball. But for older persons, especially those of sedentary occupation, exercise that exhilarates is far more beneficial than that which is not enjoyed. One may take a walk and carry all his cares and anxieties with him, but he is not likely to think of such matters when playing temnis with a good opponent. Whether it be horseback riding, cycling, boxing, boating, or other form of exercise, choose, whenever a choice is possible, that which you thoroughly enjoy.

Most of the games of school children are excellent kinds of exercise. Cases have been reported of injury from excessive skipping the rope. But in moderate degree it is a good exercise. Tag, snowballing, racing, the various games of ball, jumping, hopping, and other games may be played on the school 
grounds. Tènnis is a splendid game, and suitable for girls as well as boys. It has the great advantage over baseball that it does not require a large ground (which often means going some distance from the school grounds or from home). Two can make up a game, and a little time can be better utilized than with the games requiring more players. The exercise, too, is more evenly distributed. There is no long waiting, as in some games, but a constant interchange of play, active but not severe, with practically no danger of injury. For those who can pursue the more vigorous games of baseball and football they are admirable, and should not be objected to by teachers and parents because occasional injury comes from them. No vigorous exercise is wholly unattended by risk, though it is usually slight when the proper care is used. All these games calling for great activity and strength develop manly qualities in boys, and do much to make them active fearless men, men who in time of danger have not only strength and endurance, but well-trained muscles, cool heads, and brave hearts, men who know what to do and how to do it in an accident, as at fires, upsetting of boats, etc. A few strong, cool-headed men, by their presence of mind, often stop a panic and save many lives when there is an alarm of fire, which often proves false.

For men in middle life, in most cases, milder exercises are preferable, such as shooting and fishing and horseback riding. Every person should have some form of exercise that takes him into the open air daily. The English are more given to their "constitutionals" than their American cousins, and are the better for it. Doubtless if we paid more attention to these matters, we should lose something of our national reputation as a "nervous people." English women are noted walkers, and do not seem to pride themselves on the smallness of their feet. The signs of the times would appear to show 
that we are improving in this respect. Probably Americans make too much use of street cars. Walking is the cheapest exercise, and every one can afford to take it. For those who can afford it horseback riding is admirable. As Dr. Holmes expressed it, "Saddle leather is in some respects even preferable to sole leather; the principal objection to it is of a financial character." Lord Palnerston said "the outside of a horse is the best thing for the inside of a man." Bicycle riding is an excellent substitute. Perhaps livery bills would prove cheaper and more agreeable than doctor's' bills.

Boxing. - Boxing is a splendid exercise. It calls into play nearly every muscle of the body. Many pieces of apparatus in a gymnasium are for the especial purpose of working certain muscles. But a pair of boxing-gloves may be said to contain a whole gymnasium. Many kinds of work in a gymnasium are likely to be overdone, especially if not under the direct supervision of a good director. One may overlift or overstrain himself. But in boxing there is little tendency in this direction. Boxing makes one quick on his feet, trains to quick movements of the arms, trains the eye, keeps the body in an erect position, and especially develops the muscles of the legs and back. Cycling seems (though an excellent exercise) to make round shoulders. But boxing brings out the chest and shoulders. It developes the "wind," and keeps one in constant action. It teaches control of the temper more than almost any other form of exercise. It develops a degree of self-reliance that is worth much. Instead of developing a tendency to become involved in quarrels, it prevents getting into such disgraceful affairs. The man who knows that he can defend himself when it becomes necessary is far less likely to pay serious attention to idle bluster and slight provocation than one not so trained. And it may prove valuable to know how to defend one's self from the attack of a ruffian, or 
bully, or drunken brute, or other infuriated animal. The coolness of head, the quick judgment, and prompt action of a trained boxer frequently save one from serious injury, and add not a little to personal comfort. Like tennis, boxing calls for little apparatus, little space, and only two persons. In many places where ordinary gymnasium work is out of the question, boxing is available. It is indeed a "manly art"; and the docirine taught in Tom Brown's School Days at Rugby is as wholesome as can be given to boys to make them strong and active, to give them physical and moral health.

Dancing is, in itself, an admirable exercise. But the very exhilaration to which it owes one of its chief benefits creates a tendency to over-exercise. As usually conducted, indoors, frequently prolonged into the late hours, where the ventilation is not always looked after, and there is likelihood of taking cold afterward, this exercise has many drawbacks. If parents were wiser they would see that this very attractive amusement will be indulged in, sooner or later, by most young people; and they would, perhaps, endeavor to regulate what they cannot prohibit. By going with their children, by seeing what company and what hours are kept, even more, by joining them so far as their age permits, this beautiful exercise would prove beneficial, and would not meet the sweeping (sometimes deserved) condemnation which it now receives in many quarters.

In many of the older writings we have met the statement that sedentary persons, especially students, get a fair amount of exercise of the lower limbs in walking to and from their daily work, and that what they especially need is the exercise of the arms and the upper parts of the body. But, while this is to a certain extent true, the practice has been, in many systems of gymnastics, carried to an extreme. Lagrange, in his book, Physiology of Bodily Exercise, emphasizes the 
point that the stronger muscles ought to do the greater part of the work. The older view was that exercise requiring lifting the arms had a tendency to promote respiration, and thus to aid respiration and circulation. This is true to a certain extent. But Lagrange makes very clear that what is desired is to create what he calls a "respiratory need," that is, a demand of the system for more oxygen, together with a more active getting rid of waste matters. This is more effectively accomplished by employing the larger and stronger muscles, namely, those of the lower limbs. In the four-legged animals the greater strength and weight are in the hind limbs. And in man the lower limbs have all the work of support and propulsion of the body. The muscles of the lower part of the body, being so large and powerful, can do much more work without being overworked. Suppose, for instance, that a student has half an hour or an hour for exercise. It is evident that if he uses the muscles of the upper half of the body he cannot do anywhere near as much muscular work as if he uses the lower half of the body for the same length of time. Compare, for instance, a half hour's work with dumb-bells with the same time on a bicycle. This working of the larger muscles will much more actively stimulate respiration and circulation, and help digestion and excretion more, than the smaller and feebler muscles possibly could.

Further, in using the smaller muscles there is much greater chance of straining than in the other case. Take, for instance, work on the parallel bars. For a beginner this is very severe. It is making the upper muscles support and handle the whole weight of the body, work for which they are not intended, and to which they are not equal without a great deal of gradual preparation. One occasionally sees, in a circus, some performer who has developed the arms and shoulders out of all proportion to the other parts of the body. Nature, in the 
process of evolution, has brought man to the erect posture, and enabled him to walk on one pair of limbs. Now, for him to try suddenly to reverse this long process of development and walk on his hands, with his whole body up in the air, is doing violence to his structure, and is likely, if followed to any considerable extent, to be attended by injury. Not that the gymnastic apparatus is bad; but that; in the main, exercises that conform more nearly to the natural condition of man's upright position are more natural, and, as a rule, better. So all the games that call for running, jumping, etc., are naturally preferable to climbing, etc.

Ledger Account of the Body and its Organs. Through the digestive tube and lungs the body receives additions; and there is a corresponding loss through the lungs, skin, kidneys, and intestines. So a ledger account might be kept with the body; and it should balance in the long run, since in adult life the weight remains practically constant.

So we might take a single organ, say the liver, and balance its accounts. It receives a large amount of blood. To offset what it takes from the blood, it gives to the intestines a large quantity of bile, and to the blood it gives glycogen.

It is especially interesting to note the losses and gains of the blood as it passes through the various organs of the body. A river, flowing past one State after another, will take some of the soil of each, and deposit some of its muddy particles on the banks of each State. Of course, the blood is unlike the river, in that it empties into itself; i.e., it is truly a circulation. The blood takes something from, and gives something to, each organ as it flows through it. From the intestine the blood gets the chief part of its new material in the newly digested food. To the muscles the blood gives nutritive material and oxygen, and receives water, carbon dioxid, and other waste matters. The account would be a good deal 
the same with the brain. In the skin and the kidneys the blood has great losses and little gains.

The accompanying diagrams may help in presenting the

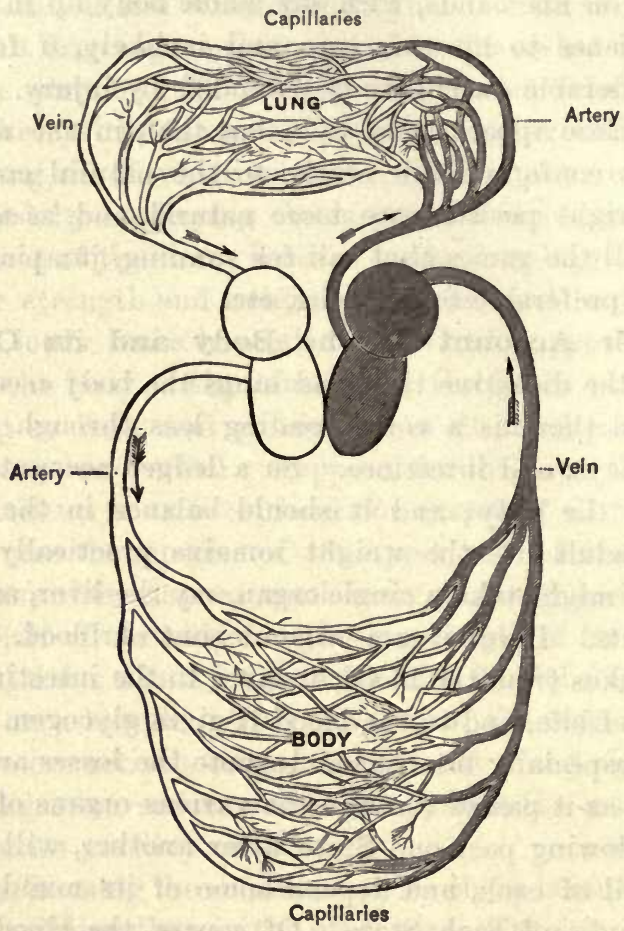

Fig. 72. Diagram of the Heart and Blood Tubes. (Dorsal Viev.)

main points in the blood circuit, and the losses and gains in its course.

In the common blood streams are combined the good and the bad. The newly digested food is received into a current of impure blood returning from the muscles (Caval Veins). 
The blood from the kidneys, probably the purest blood in the body, joins the same impure stream. From the Aorta, red blood, usually called pure, is sent to the kidneys and to the

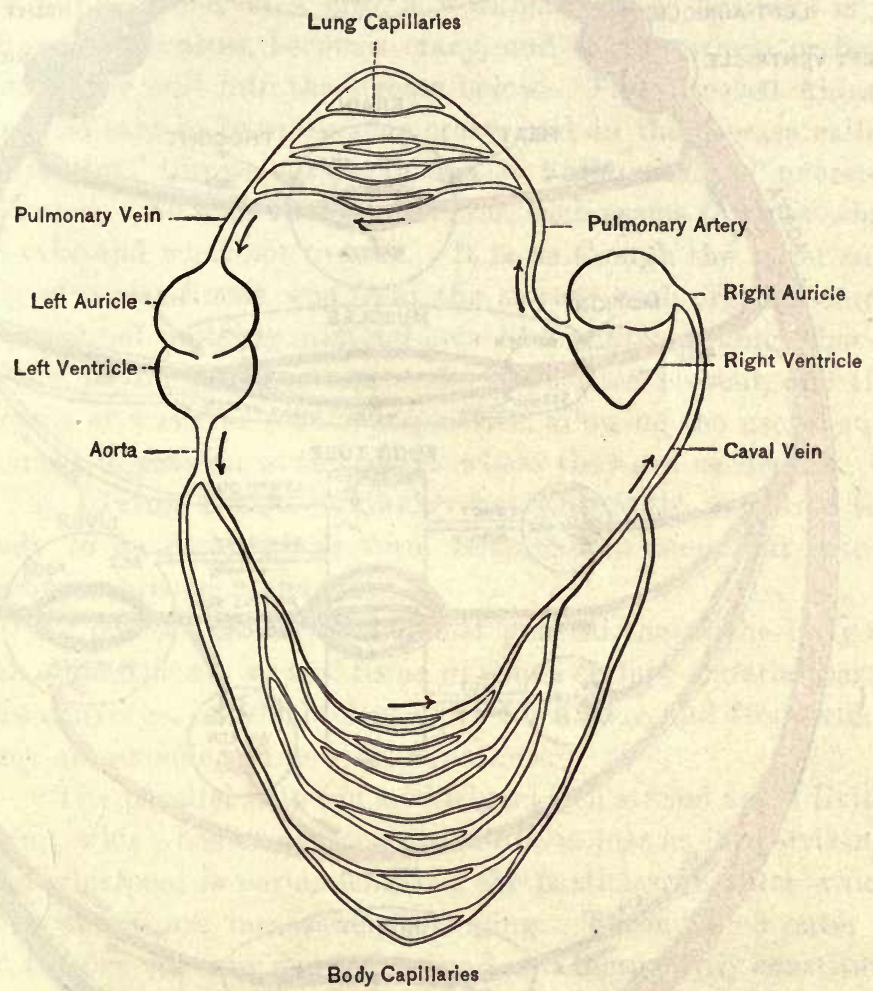

Fig. 73. Diagram of the Circulation, representing the Right and Left Halves separated, showing that the Blood makes but one Circuit. (Dorsal View.)

skin to be purified. Yet, as this mixed blood flows through each organ, that organ, so long as it is in health, takes from it only what it should take. The kidney takes, during health, 


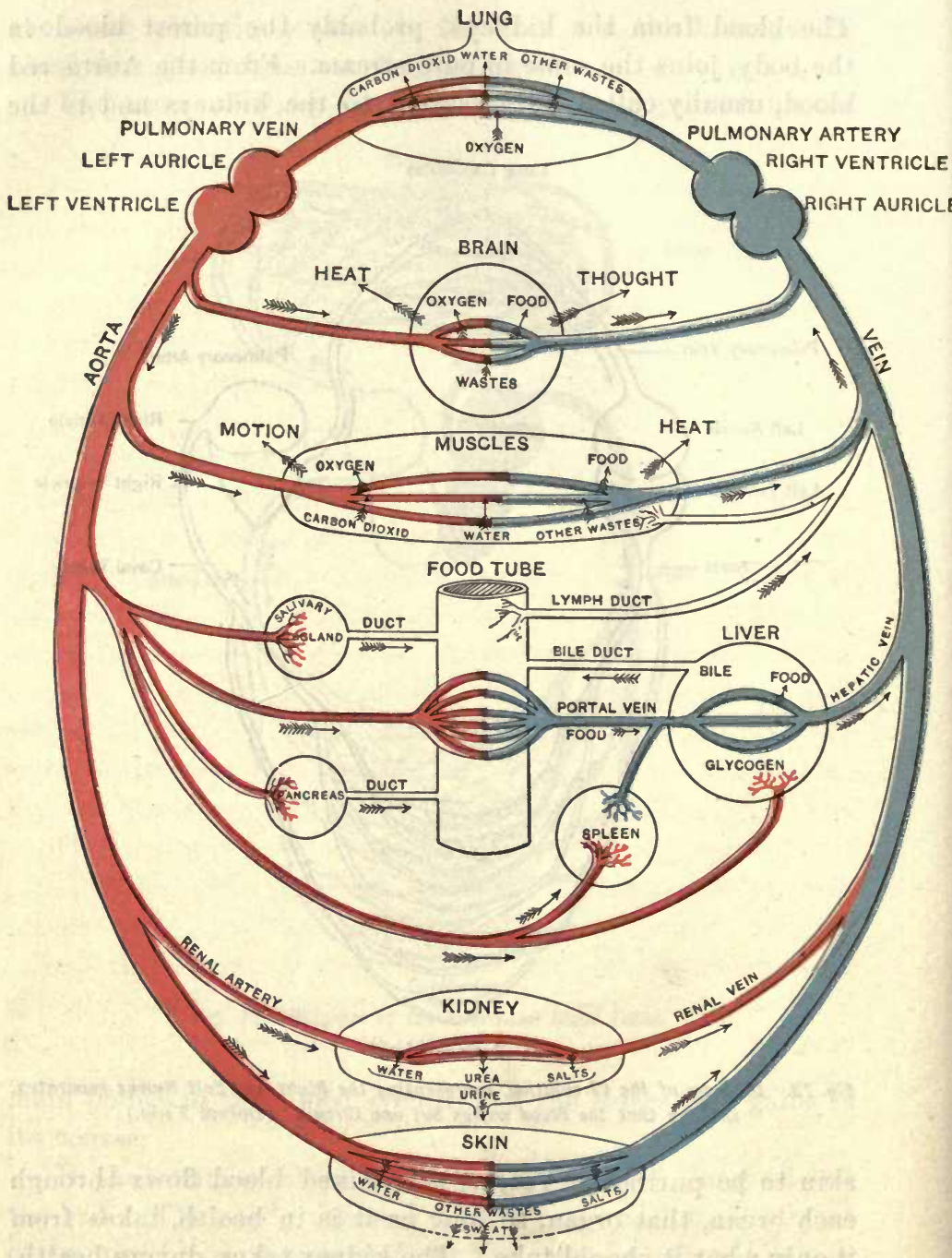

Flg. 74. Diagram of the Circulation of the Blood. 
only the waste matters, leaving the valuable nourishing material. But in disease the kidneys may throw out some of the most valuable portions of the nutriment.

Suppose that in a mill, a workman, whose business is to shovel out wastes, becomes crazy, and shovels wheat or flour out of the mill into the stream below. The diseased kidney may be said to have become crazy, and in the disease called "diabetes" throws out sugar, and in "albuminuria" excretes albumen. So with the other organs, each seems to know what to take and what not to take. It is as though the water supply of a city house was from the sewer; each organ needing a supply of building material acts like a filter, taking what it needs, paying no attention to the impurities present, and the organs of waste select the impurities, allowing the useful substances to pass on to the places where they are needed.

A Living Eddy. - Huxley has very aptly compared the body to an eddy, whose form remains the same, but whose particles are ever changing.

"To put the matter in the most general shape, the body of the organism is a sort of focus to which certain material particles converge, in which they move for a time, and from which they are expelled in new combinations.

"The parallel between a whirlpool in a stream and a living being, which has often been drawn, is as just as it is striking. The whirlpool is permanent, but the particles of water which constitute it are incessantly changing. Those which enter it on the one side are whirled around and temporarily constitute a part of its individuality; as they leave it on the other side, their places are made good by newcomers.

"Those who have seen the wonderful whirlpool, three miles below the Falls of Niagara, will not have forgotten the heapedup wave which tumbles and tosses, a very embodiment of restless energy, where the swift stream hurrying from the falls is compelled to make a sudden turn toward Lake Ontario. 
"However changeful in the contour of its crest, this wave has been visible, approximately in the same place and with the same general form, for centuries past. Seen from a mile off, it would appear to be a stationary hillock of water. Viewed closely, it is a typical expression of the conflicting impulses generated by a swift rush of material particles.

"Now, with all our appliances, we cannot get within a good many miles, so to speak, of the living organism. If we could, we should see that it was nothing but the constant form of a similar turmoil of material molecules, which are constantly flowing into the organism on the one side and streaming out on the other." - From HuxLEy's The Crayfish, as an Introduction to the study of Zoölogy as modified by Sedgwick and Wilson in their Generul Biology.

It will be well here to recall some facts noted in connection with the study of the blood and lymph. We then learned that the lymph (the supply and renewal of which depends upon the blood) surrounds the individual cells which make up the tissues of the body; and that, to a certain extent, every cell lives an independent life, each taking its nourishment directly from the lymph around it. The importance of an abundant supply of good lymph is now more apparent. If digestion is not good, or the food be insufficient or of poor quality (whether naturally or from being badly cooked), good blood cannot be made, and the lymph will not be good. The cells are more or less starved, and the general tone of the body will soon be lowered; for the health of the body as a whole depends on the average condition of the cells composing the body, just as the condition of any community depends on the average condition of the individuals of that community.

Fat. - As a tissue fat serves as a stored-up food. The camel's hump is a well-known instance. In some of the savage races fat is stored in a very similar hump. But in 
most persons it is distributed more evenly over the body, though there is a tendency to deposit rather more over the abdomen. A fat person can endure starvation longer, other things being equal, than a thin person.

Hibernating animals are fat when they enter upon their winter sleep, but are lean when they come out in the spring. Remaining inactive, they have produced very little energy, their only motions being a slow and feeble breathing and a correspondingly reduced heart-beat. They have consumed the fat, using it mainly in maintaining the necessary heat. In short, they have burned their fat to keep them warm.

In one of Captain Mayne Reid's stories (The Plant Hunters) we are told how the hunters followed a bear into a cave. At the innermost end of this very long cave they finally killed the bear. Just at this time they find that their candles are all burned out, and they are left in complete darkness, lost in the bowels of the earth. Failing to grope their way out, they are at last driven to this expedient. With what combustibles thay can gather together, including their gunstocks and some of the fat of the bear, they melt some of the fat, they use the gun barrels for molds, and take strips of their clothing for wicks, and make two long candles. With these they finally light their way out to the upper world.

Now, we have seen that when we burn a tallow candle, one of the chief products of the combustion is carbon dioxid. Another product of the burning is common water. If, then, these hunters had left this bear to his winter's nap, he would have consumed this fat in the slow process of breathing, and it would have given off the same products, as we have proved that two of the waste matters of the expired breath are carbon dioxid and water.

Glycogen. - As stated above, glycogen is formed in the liver. This is indicated by the fact that there is more sugar 
in the blood in the hepatic vein than in the portal vein, except during digestion. Glycogen is formed by and stored in the liver, and is doled out to the tissues. 'That muscles use sugar in their action is indicated in the fact that the arteries bring to the muscles more sugar than is carried away from them by the veins. As fat is a reserve food, so glycogen serves as a temporary carbohydrate reserve.

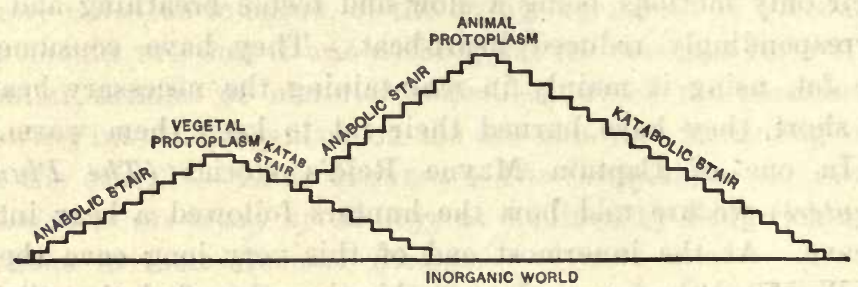

Fig. 75. Animal and Vegetable Protoplasm.

Nutrition. - All the changes that take place between the reception of food and the excretion of waste are included under the term Nutrition. The materials taken as food are usually more complex and unstable, the waste products more simple and stable; just as the products of combustion are, as a rule, simpler and more stable than fuels. In both combustion and the processes of nutrition the final result is oxidation, more or less direct. Since muscles are the engines of motion, and also are largely composed of proteid (nitrogencontaining) material, we would naturally expect that increased muscular exertion would increase the excretion of urea (the only nitrogen-containing waste). But experiment shows that increased muscular action, such as mountain climbing, hardly increases the amount of urea excreted. Such work, however, does largely increase the amount of carbon dioxid excreted. It is thought, therefore, that oir energy is largely derived from carbohydrate foods and fats; and this view is strength- 
ened by the fact that our beasts of burden depend chiefly on carbohydrate foods.

"Proteid, by digestion, becomes peptone, which is absorbed and retransformed into the proteids of plasma and lymph. These fluids surround and permeate the organized elements of the tissue, and the proteids that they carry form the floating balance of nutritive matter from which the comparatively fixed capital of living protoplasm is supplied. Of this free, circulating, or coasting proteid, in blood and in lymph, it is supposed that only a small proportion is actually taken into chemical combination in protoplasm as fixed or organ proteid, and that the greater proportion is acted upon and used by living protoplasm without being integrated by it to make part of its own substance. The free or coasting proteid thus used up gives rise to urea, as does that small proportion of fixed or organ proteid which disintegrates and gives place to a correspondingly small proportion of newly integrated proteid." - Waller.

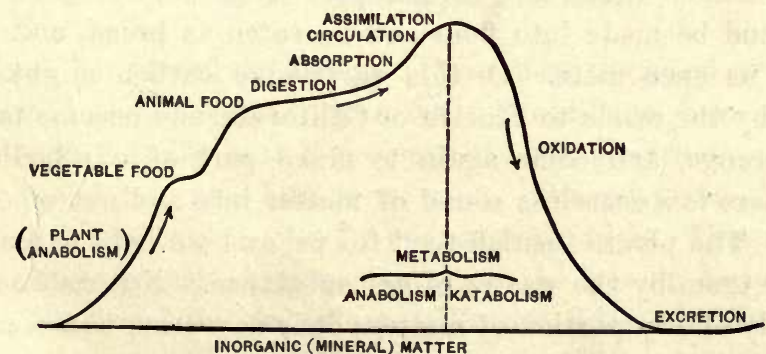

Fig. 76. Life Processes.

While increased muscular action does not very perceptibly increase the amount of urea excreted, the amount of urea is increased by increase of the amount of proteid food taken.

The building-up, or constructive, processes are included 
under Anabolism, while Katabolism designates the tearingdown, or destructive processes. All the processes of nutrition, both of building up and tearing down, are included in the term Metabolism.

The Indestructibility of Matter. - We are agreed that we cannot destroy matter. We may demolish a house, but the material is all there. We may burn it; but if we could gather the ashes and that part of the smoke and gases furnished by the material of the house, the weight would all be recovered.

In the continual wasting away of our bodies, there is no real loss of matter. Our weight is reduced; but the wastes are still part of the earth or air, and are used again. For instance, a particle of carbon in the carbon dioxid of the expired breath may be taken in through a blade of grass in an adjoining field. A cow may eat the grass, and we may soon take the very same particle of carbon in the flesh or milk of the cow. Or the carbon may be taken by that kind of grass called wheat, and become part of the seed or grain of wheat, and be made into flour and be eaten as bread, and be part of us once more. Or this particle of carbon might be carried by the winds to Florida or California, and become part of an orange, and come again to make part of our bodies. Thus there is a ceaseless round of matter into and out of our bodies. The plants furnish food for us, and we help to make food for them by the wastes of our substance. No one has a monopoly of any portion of matter; it is now ours, now someone else's. A particle may pass from one animal to another animal, as when we eat flesh or other animal food. But more often the wastes of our bodies go to make part of the air or the.soil, and are then taken by some plant before again becoming part of our tissues. But we are as unable to destroy matter as we are to create it. 
The Indestructibility of Force. - So with energy. We cannot create it and we cannot destroy it. We derive our energy from the food we eat. And this food we get directly or indirectly from the vegetable kingdom.

An engine gets energy from the combustion of fuel. In the growth of the plant under the influence of sunlight the

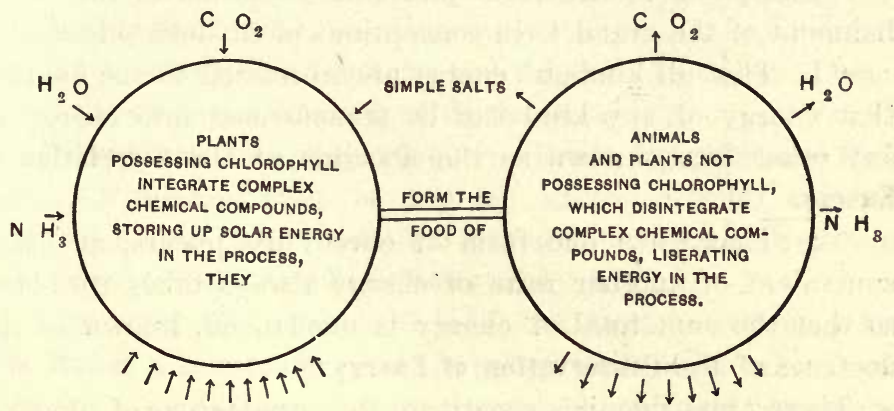

Solar Energy

Energy originally obtained from the sun radiated by the animal (chiefly) into space as heat, and thereby becoming ultimately unavailable.

Fig. 77. Relation of Plants and Animals.

plant has stored up energy. Now that the wood or coal are burned the energy is given out, primarily as heat. But we may convert the heat into electricity, the electricity into light, or back again into heat if we wish. We get our energy from food as the engine gets its energy from fuel. This is saying nothing against the superiority of the human body, and is not in the least degrading. We are self-maintaining, selfdirecting, growing, living machines. Still, starvation soon puts an end to our ability to produce energy of any kind.

Now, it is a well-recognized fact that in very many machines only the smaller part of the energy is directed to the end sought. Take a common candle. We wish to get light from it. But most of the energy of the candle is devcted 
to making heat, which, in this case, we do not desire. In many machines there is great loss from friction, loss by radiating heat, etc. Physiologists tell us that the human body utilizes a larger portion of its energy than most machines. While energy may fail to be used for the desired purpose, it is never destroyed, nor really lost.

"The proof of the facts just stated has led to the establishment of the grand twin conceptions of moderu science:-

"1. That all kinds of energy are so related to one another that energy of any kind can be transformed into energy of any other kind, known as the doctrine of the Correlation of Energy.

"2. That when one form of energy disappears, an exact equivalent of another form of energy always takes its place, so that the sum total of energy is unchanged, known as the doctrine of the Conservation of Energy."

These two principles constitute the corner stone of physical science, and must be learned and kept in mind if we would understand the actions of our bodies, and our relations to the surrounding parts of the world and the universe in which we live and of which we must consider ourselves a part.

What is the object of this ceaseless change of nutrition and growth? Is it simply that the body may be nourished? Do we live to eat, or do we eat to live? And what is it to live? Merely to be an animal, even the most highly developed on this earth?

"On earth there is nothing great but man. In man there is nothing great but Mind." The muscles are the servants of the will. These servants we have been studying. Let us turn to the study of the Master Tissue, the central nervous system.

Reading. - See Appendix. 


\section{CHAPTER · VIII.}

\section{THE BRAIN.}

The muscles are the executive organs. But the seat of the Will is the brain.

Let us study its structure and relations in order to come nearer to an understanding of its functions.

Directions for preparing the Brain of a Cat or Rabbit. - Directions have been given on page 32 for uncovering the brain. To remove the brain, it will be necessary to cut through the tough Dura Mater that covers it.

- Removing this, there will be found an inner covering, the Pia Mater, a membrane richly supplied with blood tubes, from which the brain gets its nourishment. After the dura mater has been removed, the anterior end of the brain may be gently lifted with the handle of the scalpel and the under surface studied, following the directions in finding the cranial nerves.

The brain may be studied while it is fresh, but it is more easily handled after it has been hardened. Lay the brain in weak alcohol, about twenty-five per cent. It should rest on a layer of cotton, otherwise it may be very much flattened by its own weight, and get a good deal out of shape. Later transfer it to fifty per cent alcohol, and then to seventy-five per cent, or use a solution of aicohol and formalin as follows : 95 per cent alcohol, 60 parts ; 2 per cent formol, 40 parts. The liquid need not be changed if used in sufficient volume. (See Appendix for another formula.) When it is well hardened, it may be sliced with a sharp scalpel as directed.

The Brain of the Rabbit (Alcoholic Specimen). - The 
brain of a eat or dog is better, being larger. Take a brain well hardened, and review the parts as named above. It is very desirable to have a specimen in which the arteries have been injected.

1. Press down the cerebellum, to see the deep groove between it and the cerebrum. The thin membrane covering the brain, and dipping into the grooves, is the Pia Mater.

2. Press down the spinal bulb (medulla oblongata), and tear away the pia mater where it passes from the cerebellum to the spinal bulb. Note, between the bulb and the cerebellum, a space covered by a thin membrane. Cut through this membrane; the cavity is the Fourth Ventricle of the brain. Observe the two ridges bounding the sides of the fourth ventricle. At the point of their divergence, observe the opening of the Central Canal of the spinal cord.

3. Gently separate the cerebral hemispheres, and note the transverse band of white fibers connecting them.

4. Examine the under surface of the brain, and find the roots of the cranial nerves.

The Cranial Nerves. - 1. The 0lfactory Lobes (probably cut or broken off) extend forward from the fore part of the cerebral hemispheres.

2. Note that the Optic Nerves join each other before reaching the brain. Only the first and second pairs of cranial nerves directly enter the cerebrum.

3. Back of the optic nerves, near the middle line, are the third pair of nerves.

4. The fourth pair extend up on each side into the groove between the cerebrum and the cerebellum.

5. Back of these are the larger fifth pair. This pair supply part of the face, and send branches to the teeth. It is the nerve affected in neuralgia of the face.

6. Back of and inside of the fifth pair are the sixth pair. 
7. The nerves of the seventh pair are larger, and are farther back and outward. These are the Facial Nerves, and control the muscles of the face and the facial expression.

8. Close to the seventh are the eighth, or Auditory Nerves.

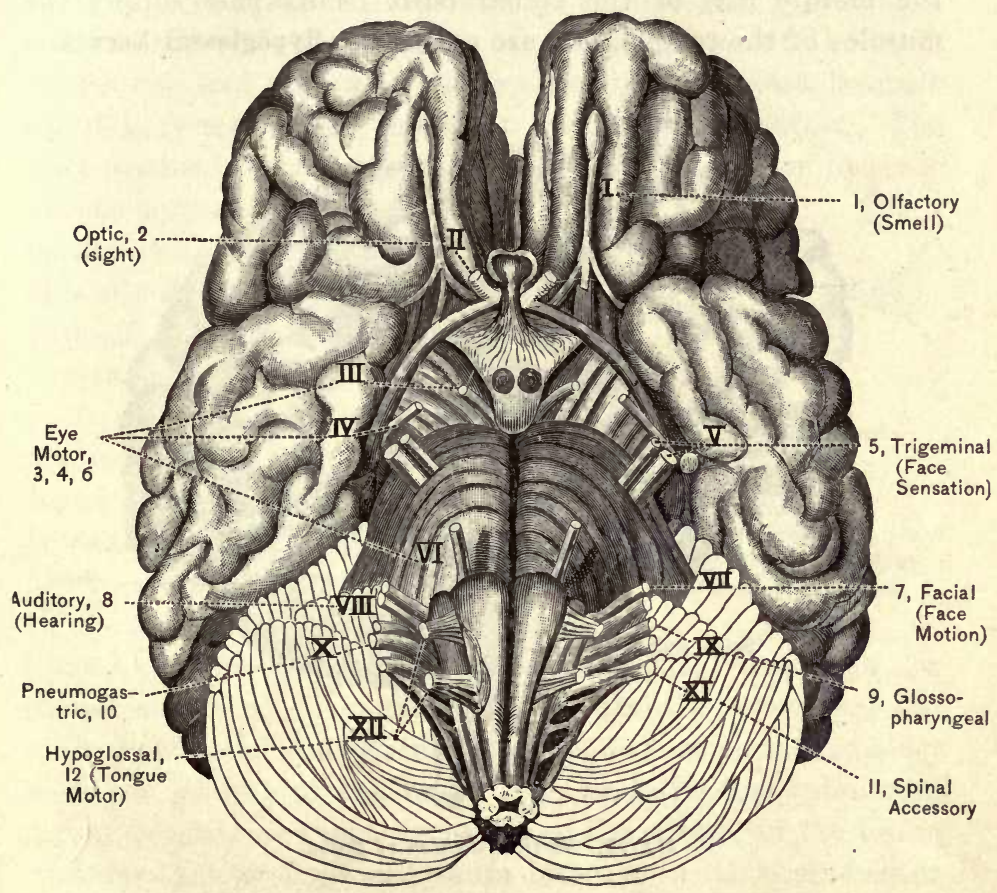

Fig. 78. The Base of the Brain, Showing the Origin of the Cranial Nerves.

9. The ninth, tenth, and eleventh arise close together, farther back and well up on the sides of the Spinal Bulb.

10. The ninth supplies the back of the tongue and the pharynx, and is called the Glosso-pharyngeal Nerve. 
11. The tenth pair pass down out of the brain cavity, give off branches to the pharynx and larynx, and are distributed to the heart, lungs, and stomach; hence the name Pneumogas. tric Nerves.

12. The last pair of cranial nerves, the twelfth, arise near the middle line of the spinal bulb. This pair supply the muscles of the tongue, and are called the Hypoglossal Nerves.

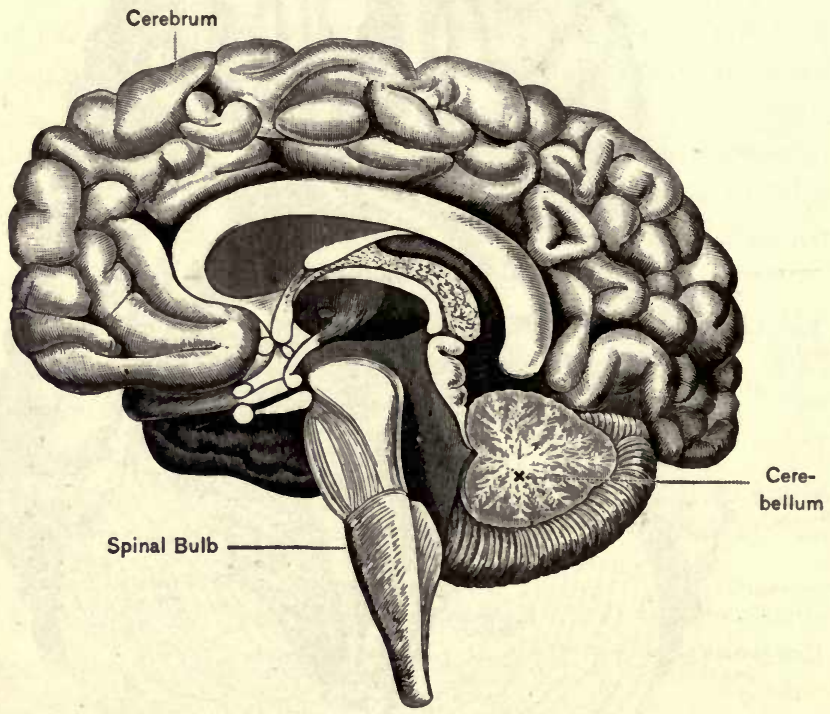

Fig. 79. Vertical Section of Brain.

Draw the brain as seen from below, showing all these nerves.

Separate the cerebral hemispheres, and with a sharp knife split the brain lengthwise in the middle line. Make a drawing of the inner face of one half. Note the branched appear. ance, the Arbor Vitæ, of the cerebellum. Trace the cavities of the brain. 
Trace the blood tubes of the brain. For this the injected brain of a cat or dog should be used.

Cut and examine the cross sections of the spinal cord after it has been hardened in alcohol. Compare the colors of the inside of the brain and spinal cord.

It will be observed that the brain, like the spinal cord, consists of two lateral halves. Cutting sections of the brain lengthwise and crosswise shows that the outer part is made up of gray matter and the inner part of white matter. The gray matter is composed of cells essentially similar to those of the spinal cord, while the white matter of the inner part is composed of white fibers like those of the outer part of the spinal cord, or like the nerves.

In comparing the brain of the rabbit and the cat, that of the rabbit is found to have fewer convolutions, and is nearly smooth. In general, the lower animals have fewer convolù-

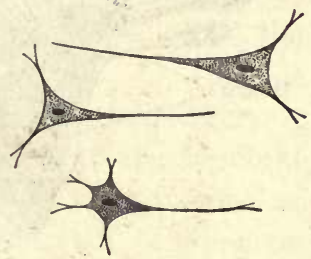

Fig. 80.

Pyramidal Nerve Cells, found principally in the Gray Matter of the Brain. tions, and the lower races of mankind have smoother brains than the higher races. In the earlier stages of development man's brain is smoother; but with growth the convolutions appear, and increase in number with the growth of the brain. As we know that intelligent action depends on the gray matter of the surface of the brain, we infer that to accommodate its increase in the brain-case it is thrown into folds, as the surface of the lining of the intestines is increased by folds, villi, etc.

The gray matter of the convolutions of the adult human brain is about one-fifth of an inch thick, the larger part of the brain consisting of the white matter. Sections will show that there are several masses of gray matter in the brain 
deeper than the convolutions. These are the Ganglia of the brain. The white fibers inside the brain connect the gray matter of the convolutions and these ganglia with all parts of the body through the spinal cord.

The brain consists of nerve-cells and nerve fibers, bound together and supported by a form of connective tissue called

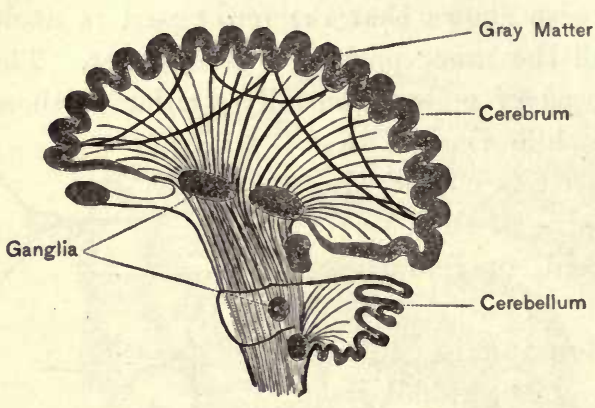

Fig. 81. Diagram of the Brain, Showing the Spinal Cord, Ganglia, and Course of the Fibers.

\section{Neuroglia,}

It will be found extremely helpful to have a set of models of the human brain. The parts are essentially the same as in the cat. Examine the base of the brain in such model, and review the cranial nerves.

The Functions of the Cranial Nerves. - 1. The first pair of eranial nerves are the 0lfactory, or nerves of smell.

2. The Optic Nerves, the nerves of sight.

3. The third pair control muscles of the eyeballs.

4. The fourth pair control muscles of the eyeballs,

5. The fifth are the nerves of sensation for most of the head and face, including the teeth. The fifth pair of nerves are like the spinal nerves in having two roots, a motor and a sensor root. Some of the fibers run to the muscles of mastication. One branch of the fifth nerve is distributed to the fore part of the tongue, and gives the sense of taste. It is called the Gustatory Nerve, or the gustatory branch of the fifth nerve. 
6. The sixth pair control muscles of the eyeballs.

7. The seventh or Facial Nerve is the nerve controlling the expression in the face as it is distributed to the facial muscles.

8. The eighth are the Auditory, or nerves of hearing.

9. The ninth are the Glosso-pharyngeal, and give the sense of taste from the base of the tongue.

10. The tenth, the vagi or Pneumogastric Nerves, extend not only to the lungs and stomach as the name indicates, but also send fibers to the heart, gullet, larynx, etc.

11. The eleventh pair arise outside of the cranial cavity, enter, and pass out again, to supply certain muscles of the neck and shoulders.

12. The twelfth pair control the muscles of the tongue.

The Cerebrum and its Functions. - If the cerebral hemispheres are removed from a frog, he will sit up about as before, but seems to pay little attention to what is going on around him. If placed on his back, he will turn over and sit up. If pinched, he may jump away, and may show that he can see by avoiding anything that may come in his way. If placed in the water he will swim, and if he swims against anything that he can climb upon, will do so and remain quiet. If placed on a board, and the board be slowly tilted, he will move along and keep his equilibrium, climbing over the end of the board if necessary to keep his balance. If left alone, he will not move; and will die in his tracks, though he will eat food if it is put in his mouth. He seems to have lost the power of Willing to do anything, or what we call the power of Volition. He originates no action.

A pigeon with its cerebrum removed acts in about the same way. It remains quiet, stupid, paying no attention to ordinary events. A sudden loud noise may cause it to start. If its tail be pulled, it moves forward to regain its balance. 
If thrown in the air, it flies for a distance. It swallows food placed in its mouth, but would starve surrounded by food. Placed on its back it will right itself, but it does not show the usual degree of Intelligence and Will-power.

"Experimentally, we learn that after the removal of the cortex (gray matter) an intelligent animal is reduced to the

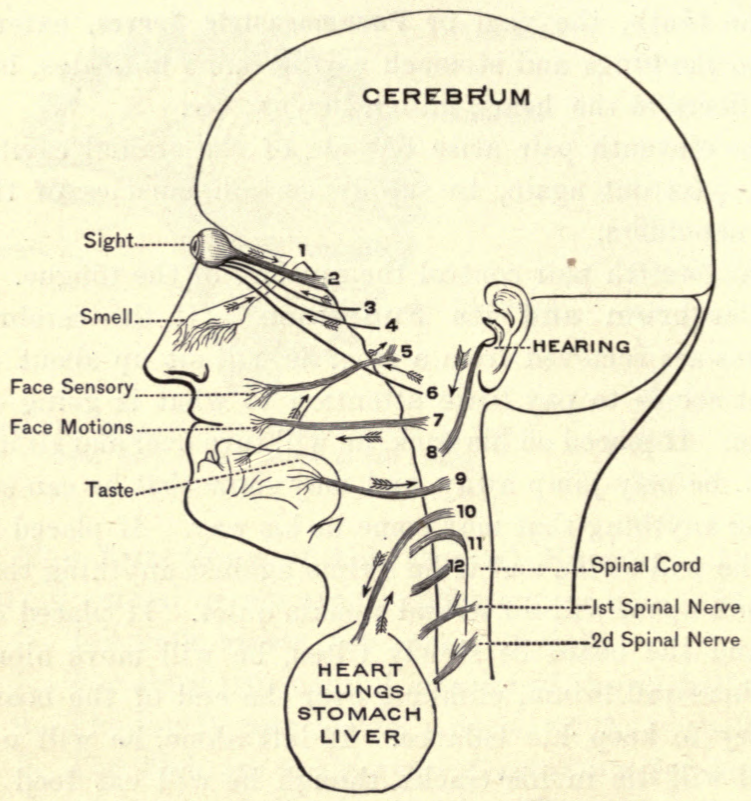

Fig. 82. Diagram of the Cranial Nerves and Sense Organs.

state of a non-intelligent automaton, responding indeed to stimuli, internal as well as external, but failing to interpret the significance of present events in accordance with bygone experience. A brainless dog is stupid; he may see a bone in front of his eyes without showing signs that he knows the 
meaning of a bone, or the use to which it may be put; he may hear the crack of a whip, but he no longer shows signs of fear, for he does not remember its sting; his former purposeful behavior has entirely disappeared; in short, he has lost memory and judgment." - WALLER.

In an earlier chapter we learned that the cortex (gray matter of the outside of the brain) is the central organ of intelligent sensation and motion. The functions of Volition, of Consciousness, of Intelligence, seem to reside in, or rather to depend upon the activities of, the cells of the gray matter of the convolutions of the cerebrum. This we have learned from experiments on the lower animals, and from accidents and disease in the case of man. All sensation seems to be in the gray matter of the convolutions of the cerebrum, and yet it is itself insensible; it may be cut and cause no sensation. But when the nerve impulses from the various parts of the body reach the gray matter of the cerebrum, they rouse the cells here to an activity that gives us what we call sensation. It is never a sensation until it reaches this part, and is properly interpreted.

While each hemisphere mainly controls the muscles of the opposite half of the body, it also, in part, has control of its own side. Paralysis of one side (hemiplegia) is due to injury of the opposite cerebral hemisphere.

Much has been learned of late year's as to the location of special functions in the brain. Many of the motor centers have been determined in the following manner: In some of the lower animals the brain has been exposed, and on stimulating certain portions with an electric current, the movements that followed were noted. In apes, "particular movements of the arm, forearm, hand, and thumb can be produced by excitation of particular spots, almost as regularly as definite notes can be sounded on a piano by touching particular keys." 
In the case of man we infer that there is a similar location, and many cases of accident and disease have helped in this work of locating the functions. But these areas are not sharply defined.

The "speech center" is in the left hemisphere; the right eye and ear, which connect with the left brain, are better de-

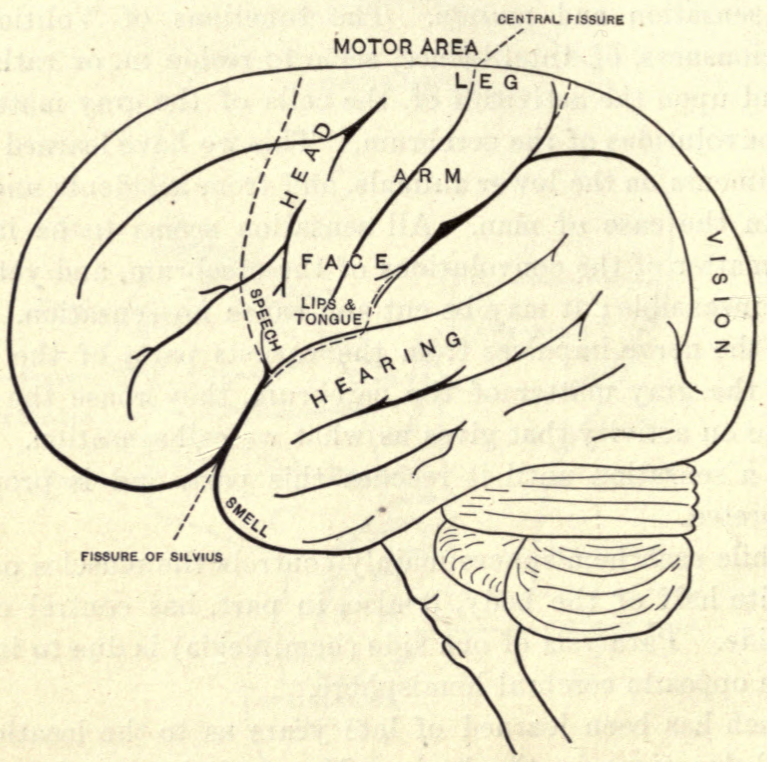

Fig. 83. Location of Brain Functions.

veloped than the left, and in general the left hemisphere seems superior (in right-handed persons) to the right.

It is not so easy to locate the centers of sensation as those for motion. For we can see the resulting motion, but a sensation can only be felt by the individual in whom it occurs. Still, some of the sensation centers have been located, and it 
is likely that in time we shall know much more on this subject.

The accompanying diagram shows some of these centers.

The Functions of the Cerebellum. - The cerebellum is the center for regulating the actions of the skeletal muscles. When we walk or run, or even stand still, a number of muscles must act, and act in concert. The nerve impulses originate in the cerebrum; but the cerebellum is the center for harmonizing the action of these various muscles, or Coördinating them. When the cerebellum has been removed from a pigeon the bird flutters, and, while possessing the power to move, does not seem capable of any regular and orderly movement. There is no loss of intelligence, no paralysis. Of course, in this experiment there is great disturbance of the system, and perhaps too much is inferred from it.

The Spinal Bulb. - The Spinal Bulb (medulla oblongata) is the connection between the spinal cord and the brain. The bulb may be said to be that part of the spinal cord which is within the cranium. It is enlarged, hence its name, Spinal Bulb. From it arise all the cranial nerves except the first five pairs. The spinal bulb is also the center for the control of many processes. It is the center for the control of respiration, of circulation, of deglutition, and perhaps for many other processes.

Blood Supply of the Brain. - Brain Work and Brain Rest. - During the period when the brain is more active, it receives a larger supply of blood. During sleep it is paler. Sleep is not merely rest for the body; it should be complete rest for the brain. In so far as there are dreams, it would seem to indicate a partial activity; that is, incomplete rest. The brain-worker especially needs plenty of sleep; excellent authorities say at least eight or nine hours. The brain, like the muscles, needs exercise; and it also needs regular periods 
of rest. If a nerve-cell is not kept active by the passage of nerve impulses through it, it usually atrophies, and may degenerate.

Intense brain work, without sufficient sleep, is likely to lead to sleeplessness, as when one has some subject of special study in hand and either will not or cannot throw it off. Perhaps inventors are as prone to this sort of trouble as any one class of men. Keeping the blood continually in the brain, or in any organ, is likely to lead to a permanent congestion or inflammation that may cause serious, if not fatal, results. It is stated that brain-workers need more sleep than those who work chiefly with the muscles. Fatigue of the voluntary muscles is much more a matter of nervous than of muscular origin. When one is completely "tired out," as he would say, if his mind can be aroused, as by some excitement, he will be found able to expend a good deal more muscular energy. So, too, many persons of slight muscular build, but of great "will-power", are able to do more work with the muscles than others with larger muscles and less will.

But the brain-worker should not only be able to sleep regularly and long enough, he ought to be able to throw off his mind any subject, and take rest while he is awake. If one allows himself to think about mental work while eating, the process of digestion will not go on well.

The student should acquire the power, and cultivate the habit, of having, so far as possible, regular hours for work, and of completely throwing aside his work and worry at stated times. In seeking recreation it is well to choose that which will necessitate giving the attention to something entirely different from the daily work. For this reason chess may be no real recreation for the student, while a game of tennis, boxing, or other competitive exercise is likely to accomplish this very desirable object. A walk may put the 
muscles into play; but if the mind is still intent upon the line of work maintained throughout the day, the exercise may prove of little benefit. He may return more tired than when he set out. The exhilaration of horseback riding may prove far better, though perhaps involving much less muscular exertion.

During fatigue the cell-bodies are found to decrease in size, but there is no discernible change in nerve fibers as a result of fatigue.

It is worthy of note that in fasting the nervous tissue is less reduced than any other tissue, being scarcely diminished by complete starvation.

Blood is supplied to the brain through four arteries, - the right and left Internal Carotid arteries, and the right and left Vertebral arteries. These arteries are so connected by cross branches that if any three of them should be compressed, or the blood-flow in them otherwise stopped, the fourth would still be able to give the brain blood enough for its work.

If the supply of blood to the brain is shut off, unconsciousness quickly follows. In the ordinary faint the blood supply has been reduced, owing to the diminution of the blood pressure or heart's force. It may be due to inhibition of the heart from some emotion, or bad odor, as in a close room; severe pain may be the cause; a blow over the pit of the stomach may stop the heart by reflex action. Fresh air should be supplied, and the body laid flat on the back. This position makes it easier for the blood to reach the brain and restore consciousness. Smelling salts (or ammonia) may stimulate respiration and circulation. Sprinkling a little cold water on the face may have the same effect, but it is not necessary to pour a large quantity of water over the person. Rubbing the limbs toward the heart promotes the flow of blood, and tends to start the heart to activity. 
Apoplexy is caused by rupture of a blood tube and the formation of a clot that presses on the brain. In meningitis there is inflammation of the membranes immediately surrounding the brain or spinal cord or both.

Between the coats surrounding the brain and spinal cord there is a layer of liquid, comparable to that around the heart or lungs. When an undue amount of blood is sent to the brain it is supposed that part of the cerebro-spinal fluid is pressed out into the spinal cavity, thus relieving the pressure in the brain cavity.

The gray matter is physiologically more active than the white, and in keeping with this is the fact that the capillary network is closer in the gray matter than in the white. This is true of the spinal cord as well as of the brain.

GENERAL CONSIDERATIONS CONCERNING THE NERVOUS SYSTEM.

Nerve Stimuli. - Natural nerve impulses that run outward are ordinarily started by the action of some nerve cell or cells, as from the gray matter of the brain or of the spinal cord.

Nerve impulses coming inward may be started in several ways. Ordinarily by some one of a few forces that are capable of affecting the nerve endings.

Mechanical force, as pressure, acts on the nerve endings of the skin, and starts nerve impulses which are carried to the brain and rouse certain cells to activity, and give us the sensation of touch. The vibrations known as light excite the special nerve endings in the retina, but affect no other nerve endings. Sound is appreciated only by the endings of the auditory nerve. Certain gases or fine particles affect the olfactory nerve endings, and certain substances may give 
the sense of taste by acting on the ends of nerves in the mouth. Different nerve endings then, are adapted to receiving impressions from the action of different forces.

Nerve endings in different parts of the body may be affected by the blood and the lymph, and give us sensations of comfort or discomfort, restlessness, fatigue, faintness, etc. These are called General Sensations. They are probably due to the condition of the blood, or to the condition of nutrition of the various parts of the body. Thus after muscular exercise the muscles are acid in their reaction, while they are alkaline after resting; after exercise carbon dioxid accumulates in them to a certain extent. Hunger and thirst come on after abstinence from food and drink, or after work exhausting the tissues. The presence of the various waste products, or the condition of the cells as the result of their activity, acting through the nerve endings, in the tissues, keep the nerve centers informed as to the condition of the parts of the body. If these conditions are extreme we may have definable sensations ; but ordinarily the sensations are of an undefinable sort, which we designate as "general sensations."

In experiment electricity is usually the best stimulus; mechanical stimuli, as used in the experiments with the muscle-nerve preparation from the frog, by cutting or pinching the nerve, may be employed; heat, as in touching the nerve with a hot wire, or Kolding a hot wire near the nerve, may be used as a stimulus; chemical stimuli, as acids, strong salt solution, etc., may also be used.

It is to be noted that while special stimuli act on specially modified nerve endings, all nerve fibers are essentially alike; and the nerve impulse, however started, is probably the same kind of force. For instance, cutting the optic nerve, or severe shock, as a blow on the head, causes a sensation of light not quite so definite, but essentially the same as though light had 
acted on the retina, and thus started the nerve impulse, instead of a mechanical stimulus acting on the nerve fibers between the retina and the brain.

If we apply a stimulus of a given intensity, as of an electric current, whose intensity can be measured, it causes a sensation of a certain degree. Doubling the stimulus, or increasing it by a definite amount, does not increase the intensity of the sensation to the same degree. The sensations do not increase at the same rate as the stirnuli. To increase the sensations arithmetically, the stimuli must increase geometrically.

"Reaction time" is the time between the application of a stimulus and the signal given as a response to show that the stimulus has been "felt." Thus a blindfolded person gives a signal as soon as he is touched. This interval between the stimulus and response varies with the individual, mode of stimulation, health, attention, etc. It is from one-tenth to one-fifth of a second; is shortest for touch; longer for sight than for hearing. The total reaction time is occupied by (1) the time of conducting the nerve impulse to the brain, (2) the time occupied in the cerebral cortex in the perception of the sensation and the formation of the volition, (3) the time of conducting the motor impulse and giving the signal. The greater part is in the middle interval; i.e., the central elaboration, during which the entering impression gives rise to an outgoing impulse.

In a previous diagram of reflex action, a single cell was represented as receiving the afferent impulse and sending out an efferent one. It is more probable that at least two cells are concerned in such an act, one receiving the incoming impulse, and influencing, by means of fine connecting branches, a second cell which sends out the motor impulse, as shown in Fig. 84.

We have seen that the brain functions are more or less 
localized. We also know that the cortex receives impressions through the channels of the different sense organs, and we can respond through various channels, - speech, writing, facial expression, etc. We would therefore expect, theoretically, that the various parts of the cortex of the brain are connected. As a matter of fact, we find anatomically that this is the case. Not only are the cells of the gray matter connected with the

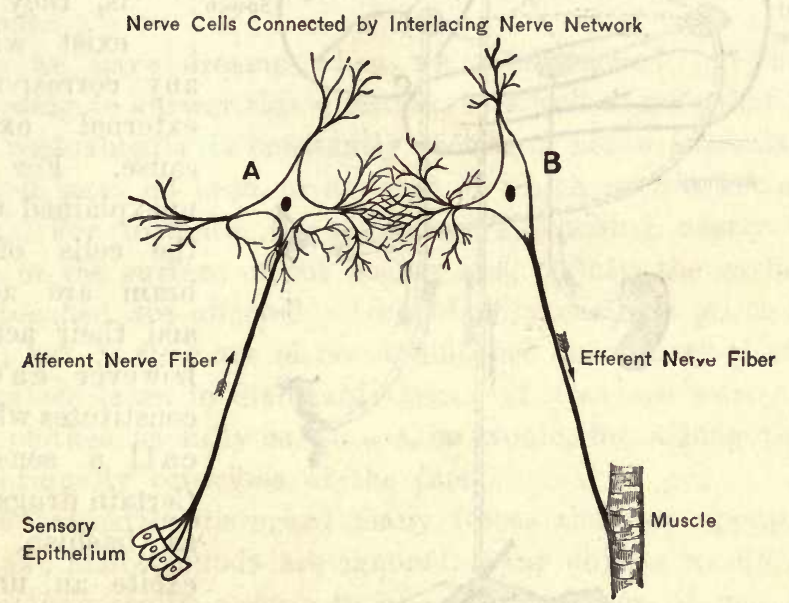

Fig. 84. Diagram of Reflex Action.

various parts of the body, but cells of different parts of the cortex are in communication with each other by what are called "association fibers." Thus a sensation roused in one part of the brain gives rise to the sending out of an impulse from another part of the brain to produce the response.

The Nature of Sensation. - Of the real nature of sensation we know but little. Like consciousness, we call it a condition of the gray matter of the cerebral convolutions. Perhaps 
the most practical definition of sensation that we can give is that it is the interpretation that the cells of the gray matter of the brain give to the nerve impulses that come from without.

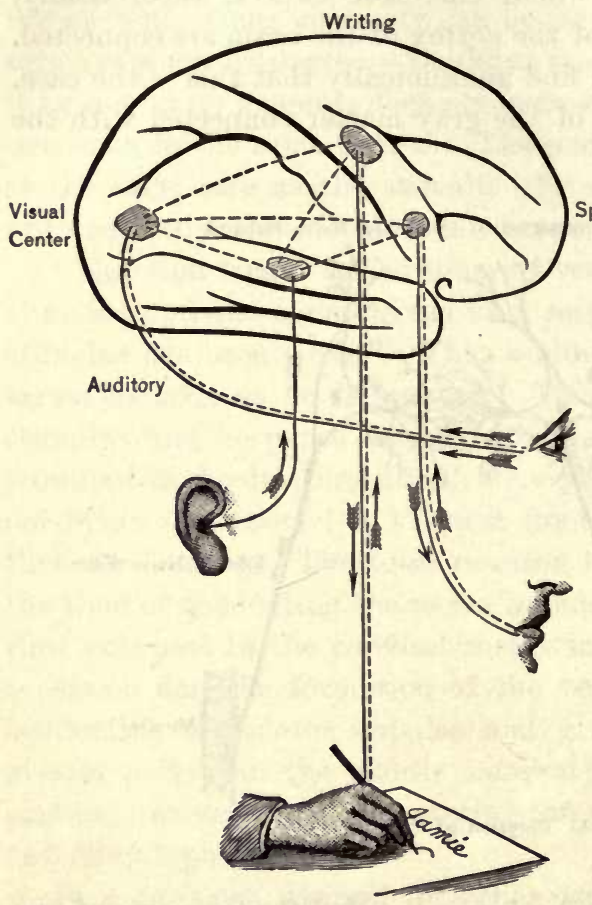

Fig. 85. Connection of Brain Centers by Association Fibers. (After Landois \& Stirling.)

(The dotted lines from the hand, mouth, and eye represent afferent fibers from the skin, muscles, and joints of the hand, lips, orbit, etc.)
This will apply to ordinary sensations. But sensations may be subjective; that is, they may exist without any corresponding external exciting cause. For some unexplained reason the cells of the brain are active; and their activity, however caused, constitutes what we call a sensation. Certain drugs, such as hashish, may excite an unusual degree of cerebral activity. Here the action is roused through afferent nerves, but through unusual channels; that is, the subject sees, but not through the nerves of sight. Many Hallucinations are explainable to a certain degree; others we may not account for. 
Dreams, due to more or less perfect brain activity, are often traceable to nerve impulses brought from the digestive. tract, from the respiratory organs, from the skin (heat and cold and pressure), from any internal organ, according to the condition of the blood, pressure, sound, etc. It seems to be well settled that dreams seeming to cover long periods of time are really gone through with in a very short space of time, just as sometimes during waking hours thoughts fly through the mind in countless numbers and with incredible swiftness.

Do we have dreams when we recall none? Without attempting to answer this question, it is well to note that the brain undoubtedly is constantly receiving nerve currents to which it pays no heed, or at least of which we are not conscious. For instance, our clothing is touching nearly the whole of the surface of our bodies, and, plainly, the surfaces thus touched are affected. Undoubtedly currents go to the brain; but, as they are of no significance in ordinary circumstances, we learn to disregard them. If a savage were suddenly clothed as fully as we are, he would, for a long time, be continually conscious of the fact.

We ordinarily disregard many forces that are operating upon us; many sounds are ignored, many objects we do not see as they really appear to us. For instance, if one sits behind a bald-headed man in a church, in looking over the top of his head at the choir, the top of his head appears peaked, because while the eyes are focused on the more distant object, the images of the man's head, as seen by the two eyes, do not coincide, but overlap each other; and what is seen is the part where the two images overlap, which forms a pointed arch. We may be sure this is what we always see, but seldom notice. And this is only a sample of the many appearances and sensations that we have learned to ignore. To 
an infant they are just as apparent as the phenomena to which we, in later life, learn to attach meaning and significance.

The Relative Nature of Sensations. - If one hand be held in a basin of hot water and the other in a basin of cold water, and then the two be suddenly plunged into a third basin containing tepid water, a sensation of cold will be received from the hand that was in the hot water, while the hand from the cold water will feel heat. Sensations depend on comparison and contrast. After listening to low sounds, a sudden loud noise is painful; and after hearing loud noises, it is difficult to detect slight sounds. We hardly notice the gradual fading of the light at sunset. And the nose does not usually detect the slow fouling of the air in a room; but let one come in from the fresh outside air, and the contrast is striking. A constant current of electricity usually causes a muscular contraction at the time the current enters the muscle and at the time when the current is stopped, that is, at the "making" and the "breaking" of the current; but the muscle ordinarily remains inactive while the current is passing. The Interrupted Current, or Induction Current, is therefore commonly employed as a stimulus in physiological experiment. A sudden change seems to be requisite for producing the nerve impulse necessary to rouse a sensation in ordinary circumstances. Pressure may be applied so gradually that we fail to notice it. The art of the pickpocket, of the ventriloquist, of the sleight-of-hand performer, depends largely on this fact. Attention is called to something else, and the work is either quickly done when attention is completely absorbed on something else, or the act is so gradual that no sudden change is noted. In smelling it is often necessary to sniff; the sudden rush of particles of air bearing the odorous particles against the surface bearing the nerve endings seems to be necessary. Perhaps this is why the fish has two nasal openings on each 
side; namely, that there may be a decided current of water over the nerve endings; for it is found that if the human nostrils be filled with rose-water no odor is perceived.

In what is called Aristotle's experiment, the experimenter crosses the first and second finger, and feels an object with the fingers thus crossed and eyes shut. If a marble be rolled about by the two fingers thus crossed, it seems to be two. Here we use Judgment with the sensation. Ordinarily we could not feel one simple solid object with the outside of the first and the inside of the second finger at the same time. This illustrates how we are constantly using our judgment in interpreting our sensations. We see few things as they are in themselves. We see nearly everything in the light of past experiences.

We have noted the lingering effects of sensations, how sights and sounds linger and are fused one with the other, so we get continuous light from a series of flashes if they follow each other in sufficiently rapid succession, and continuous sound from a series of sounds that would be heard separately if they are more than about a sixteenth of a second apart. So with touch, if the finger be held against the teeth of a revolving wheel, if the wheel revolve slowly, the touch of each tooth may be felt, but when it whirls more rapidly the sensation becomes that of continuous pressure. And it is of vastly greater significance that the cells whose activity gives consciousness - that a more permanent effect still is produced; so that we may recall, in the act called Imagination, the sensation that was originally caused by the action of some external object, through the nerve-ending and the nerve, upon the nerve-cells in the brain. Experience and experiment both go to show that probably nothing is wholly forgotten. Whatever acts upon a cell of nervous matter mákes its mark. It may become dim, but it is never completely obliterated. 
The testimony of persons rescued from drowning, and other similar experiences, goes to show that the record was yet in the mind. We may fail to Recollect, but we ever remember.

\section{EFFECTS OF ALCOHOL ON THE NERVOUS SYSTEM.}

[Treatise on Hygiene, STEvexsox and MURPHY.]

"The physiological effects of alcohol which have been considered are quite subsidiary to its effects on the central nervous system, as there is no doubt that it is for this effect on the brain that alcoholic beverages are so universally taken by mankind. The first effect that alcohol has on the brain is that of a stimulant, and it probably acts as such in two ways; namely, by increasing the circulation of blood through the brain, which is thus roused to greater vigor, and by directly stinulating the nerve-cells of the nerve centers. This stimulating effect is observed chiefly after medium or dietetic doses; and its result is seen in many individuals by an increase of mental and bodily activity, and of acuteness of perception by the special senses. This beneficial physiological effect is, however, soon replaced by poisonous symptoms if the dietetic doses are too often repeated, or a large quantity of alcohol is taken at once; for alcohol then becomes a depressant and paralyzer of the central nervous system, and symptoms of intoxication appear. This depressant effect is, as Brunton points out, one of progressive paralysis. The higher centers of the brain are first affected, then the lower. The perceptive centers are paralyzed, so that correct judgment is no longer possible, while the emotions are uncontrolled and thrown out of working gear, fits of boisterous hilarity and of emotional depression being common symptoms. Speech becomes disordered, and symptoms of incoördination, due probably to an effect on the cerebellum, appear. The respiratory center in 
the medulla then becomes affected; and at this stage there is coma with stertorous breathing, while the action of the heart still continues, even after respiration has stopped. There can be no question that alcohol taken in sufficient quantities to depress the higher centers of the brain does an infinite amount of harm."

Dr. Crothers, author of Diseases of Inebriety, says, "I have often been made impatient in listening to the lecturer presenting the 'scientific aspects of the alcohol question' to an audience, to see him illustrate extensively with charts, and spend hours to show the effects of alcohol upon the coats of the stomach, and upon the structure of the liver and the kidneys, and never allude once to the brain; when the fact is, alcohol's principal effect is upon this organ, and the functions of this organ so far transcend the functions of all the others, that, I might say, there is no comparison."

Some authors hold that the alterations in the tissues by alcoholic drinks result from the injury to the nerve centers that preside over these tissues; for their nutrition depends not merely on the direct effect of the blood and lymph supply, but also upon the direct influences of the nerve centers; they even go so far as to maintain that there is a special set of nerve fibers devoted to the control of the nutrition of the cells, and these nerve fibers they call "trophic nerves" or "trophic fibers."

"It is clear that the nervous centers, independently of the ill effects on their nutrition by the blood changes, have a certain chemical attraction for alcohol, which accordingly is found in their tissue."-Crothers.

Dr. Crothers, in common with many physicians, regards inebriety as a disease.

Dr. Clum, in his work entitled Inebriety, its Causes, its Results, its Remedy, says, "The most important part of man 
is his nervous system; the cerebrospinal, sympathetic, and vasomotor being intimately interwoven and connected, composing the whole. The great nervous center, the brain, with its hemispheres, its gray and white matter, is the most complex of all complexities. The nerve fibers not only connect every cell with every other cell, but unite all nervous structures into one, making the entire body a complete whole, and forming close and direct sympathy between the intellect and the physical organization.

"The mind and body are so intimately connected that exhausting excess of either acts and reacts on the other. Excessive work, either intellectual or physical, the sudden loss of property, intense disappointment, great trouble, unrequited affections, etc., may impart a shock to the senses through the mind, which, extending to the molecules of the brain, disturbs their normal action; and a sufferer thus worn and debilitated with the cares of life, with an enfeebled will-power, the result of nervous exhaustion, experiences a craving for some form of stimulant to 'brace him up.' $\mathrm{He}$ is on the verge of inebriety, or of insanity, or both; and if he indulges in alcoholic beverages he becomes an inebriate. Any disease inherited or acquired, acting either directly or indirectly upon the nervous system, may act as the predisposing, exciting, or complicating and protracting cause of alcoholic inebriety.

"Inebriety is often, too often, observed to flourish in the richest and most promising soil. The clergyman, the lawyer, the editor, the student, and all others who use their intellectual faculties to excess, as well as the mechanic, the laborer, and those who excessively exert their physical system, have unnatural longings for something to restore the exhausted energies of mind and body.

"The excessive worry of one man, the exhausting excesses of another, and the overwork of others, lead to organic lesions 
and nervous defects, and the disease inebriety, an ungovernable craving for alcoholic drinks, is the result.

"When a man drinks to excess, even though forced to do so by a diseased nervous system, Christian communities usually brand him as a criminal, as an outcast, and say, 'We have no sympathy for you; stop drinking and be a man,' when in reality the man should be cared for, and treated as other diseased human beings. The fact that the desire for alcoholic drinks is often a disease which may be either inherited or acquired is overlooked by those who condemn the drunkard. Our ancestors have for ages been addicted to habits of intoxication; and we, their descendants, are tainted with the disease inebriety."

\section{MORAL DETERIORATION PRODUCED BY ALCOHOL.}

[FROM MARTIN's The Human Body and the Effects of Narcotics.]

"One result of a single dose of alcohol is that the control of the will over the actions and emotions is temporarily enfeebled; the slightly tipsy man laughs and talks loudly, says and does rash things, is enraged or delighted without due cause. If the amount of alcohol be increased, further diminution of will-power is indicated by loss of control over the muscles. Excessive habitual use of alcohol results in permanent over-excitement of the emotional nature, and enfeeblement of the will; the man's highly emotional state exposes him to special temptations, to excesses of all kinds, and his weakened will decreases the power of resistance; the final outcome is a degraded moral condition. He who was prompt in the performance of duty begins to shirk that which is irksome, energy gives place to indifference, truthfulness to lying, integrity to dishonesty; for even with the best intentions in making promises or pledges there is no strength of will to keep them. 
In forfeiting the respect of others, respect for self is lost and character is overthrown. Meanwhile the passion for drink grows absorbing; no sacrifice is too costly which secures it. Swift and swifter is now the downward progress. A mere sot, the man becomes regardless of every duty, and even incapacitated for any which momentary shame may make him desire to perform.

"For such a one there is but one hope, - confinement in an asylum, where, if not too late, the diseased craving for drink may be gradually overcome, the prostrated will regain its ascendency, and the man at last gain the victory over the brute."

\section{NARCOTICS.}

Gould's Dictionary of Medicine, one of the very best authorities, thus defines narcotic: " a drug that produces narcosis," and narcosis, as "the deadening of pain, or the production of incomplete or complete anesthesia by the use of narcotic agents, such as the use of anesthetics, opium, and other drugs." It is common, however, to treat of chloroform, ether, chloral hydrate, etc., in a group by themselves under the designation Anesthetics.

The Century Dictionary thus defines narcotic: "a substance which directly induces sleep, allaying sensibility and blunting the senses, and which, in large quantities, produces narcotism or complete insensibility. Opium, Cannabis Indica, hyoscyamus, stramonium, and belladonna are the chief narcotics, of which opium is the most typical. Direct narcotics ... either produce some specific effect upon the cerebral gray matter, or have a very decided action on the blood-supply of the brain."

Some authorities class alcohol with the narcotics. 


\section{OPIUM.}

Opium is the dried and thickened juice of the head, or capsule, of a species of poppy. Incisions are made in the partially ripened heads; the milky juice exudes; after about twenty-four hours the partially dried and thickened material is scraped off with a dull knife. Most of the opium comes to this country from Smyrna, with a smaller quantity from Constantinople. As gathered it is a reddish-brown, sticky substance of peculiar odor. It is soluble in water, alcohol, and dilute acids, to all of which it gives a deep brown color. It is a very complex substance; but the chief constituent is morphia, or morphine, to which the properties of opium are due. One-fourth of a grain of morphine is equal to a grain of opium of the average strength. "Opium was known to the Greeks, but was not much used before the seventeenth century; at present it is the most important of all medicines, and its applications the most multifarious, the chief of them being for the relief of pain and the production of sleep. Its habitual use is disastrous and difficult to break up. It is classed as a stimulant narcotic, acting almost exclusively on the central nervous system when taken internally; in large quantities it is a powerful narcotic poison, resulting in a coma characterized by great contraction of the pupils, insensibility, and death." - Century Dictionary.

The United States Dispensatory makes the following statements as to its medical properties and uses: "Opium is a stimulant narcotic. Taken by a healthy person in a moderate dose, it increases the force, fullness, and frequency of the pulse, augments the temperature of the skin, invigorates the muscular system, quickens the senses, animates the spirits, and gives new energy to the intellectual faculties. Its operation, while thus extending to all parts of the system, is 
directed with peculiar force to the brain, the functions of which it excites sometimes even to intoxication or delírium. In a short time this excitation subsides; a calmness of the corporeal actions, and a delightful placidity of mind succeed ; and the individual, insensible to painful impressions, forgetting all sources of care and anxiety, submits himself to a current of undefined and unconnected but pleasing fancies, and is conscious of no other feeling than that of a quiet and vague enjoyment. At the end of half an hour or an hour from the administration of the narcotic, all consciousness is lost in sleep. The soporific effect, after having continued for eight or ten hours, goes off, and is often succeeded by more or less nausea, headache, tremors, and other symptoms of diminished or irregular nervous action, which soon yield to the recuperative energies of the system; and, unless the dose is frequently repeated, and the powers of nature worn out by over-excitement, no injurious consequences ultimately result. Such is the obvious operation of opium when moderately taken; but other effects, very important in a remedial point of view, are also experienced. All the secretions, with the exception of that from the skin, are in general either suspended or diminished; the peristaltic motion of the bowels is lessened; pain and inordinate muscular contraction, if present, are allayed; and general nervous irritation is composed, if not entirely relieved."

\section{HASHISH.}

The following quotation from Bayard Taylor is given to show the great influence of a narcotic on the nervous system. For a detailed account of the effects of this drug read the chapter entitled "The Visions of Hasheesh" in The Lands of the Saracen. "The use of Hasheesh - which is a preparation of the dried leaves of the Cannabis indica - has been 
familiar to the East for many centuries. During the Crusades it was frequently used by the Saracen warriors to stimulate them to the work of slaughter; and from the Arabic term of ' hashasheën,' or eaters of Hasheesh, as applied to them, the word 'assassin' has been naturally derived."

It is said that burglars, train-robbers, etc., often drink before their work to "nerve" them for it, being careful, however, not to drink too much.

Habits. - It is well to here emphasize that Habits are Acquired Reflex Actions.

The work of the spinal cord is that of a subordinate officer, whose duty is to relieve his superior, the brain, of many small tasks, and to afford him relief from having all the details constantly on his mind. If we learn to do many things mechanically, we save the effort of doing them by conscious effort and act of will. Whatever we do for the first time requires careful attention. To learn any new muscular action, such as a new step in marching, fingering a musical instrument, or typewriting, requires effort; they produce more or less fatigue. Subsequent effort in doing the same thing is very much less, showing that, in many cases, fatigue is mental rather than muscular. What we do from habit, and cheerfully, is easily done. Hence the desirability of forming good habits, that we may, without unnecessary effort, that is, without loss of energy, do what is needed for our wellbeing. The habit of Resting, early formed, is of incalculable value to any person, especially to the student.

The student will do well to read such books as Call's Power Through Repose, and Brackett's Technique of Rest. They are very suggestive of ways of economizing energy.

We are not conscious of expending energy in standing, until we begin to be weary; but the fact that a blow on the head causes one to fall, reveals the fact that the brain is 
constantly sending messages to the muscles to make them act. 'The shock of the blow has stopped the sending forth of these messages, and so the body is no longer supported. None of the muscles that support the body have been injured or even touched.

In studying the muscles, the fact was noted that the muscles are always slightly "on the stretch." This means that nerve impulses are continually passing from the central nervous system which tend to keep the muscles in a state of slight contraction.

We have, in youth, such a boundless store of energy that we do not sufficiently consider these matters. But if one wishes to follow the intellectual life long and successfully, he must learn to economize energy, and to direct his forces into useful channels. And one important part of this knowledge is learning how to rest. It is an art that very few have well learned.

The brain is like a telegraph office in both receiving and sending out messages. Unlike the telegraph office, it has one set of fibers to bring currents in (afferent), and another to carry currents outward (efferent).

We have concerned ourselves thus far chiefly with efferent nerve fibers and efferent currents. By way of review it may be stated that these efferent currents are sent mainly to muscles, to make them shorten or to relax, or to gland-cells to control their activity. The only other efferent currents, so far as known, are those which possibly go to the cells of the tissues to regulate their nutrition or their heat production.

Having given so much attention to the outgo of nerve impulses, let us ask the question, "What about the incoming nerve currents?"

"All life long the never-ceasing changes of the external world continually break as waves on the peripheral endings 
of the afferent nerves; all life long nervous impulses, now more, now fewer, are continually sweeping inward toward the center; and the nervous metabolism, which is the basis of nervous action, must be at least as largely dependent on these influences from without as on the mere chemical supply furnished by the blood. We must regard the supereminent activity of the cortex, and the characters of the processes taking place in it, as due not so much to the intrinsic chemical nature of the nervous substance which is built up into the cortical gray matter, as to the fact that impulses are continually streaming into it from all parts of the body; that almost all influences brought to bear on the body make themselves felt by it. To put the matter in a bald way, we may ask the question, what would happen in the cortex if, its ordinary nutritive supply remaining as before, it were cut adrift from afferent impulses of all kinds? We can hardly doubt but that volitional and other psychical processes would soon come to a standstill, and consciousness vanish. This is, indeed, roughly indicated by the remarkable case of a patient whose almost only communication with the external world was by means of one eye, he being blind in the other eye, deaf of both ears, and suffering from general anæsthesia. Whenever the sound eye was closed he went to sleep." - Text Book of Physiology, Foster.

Let us turn from the consideration of outgoing, or efferent, nerve impulses and their resulting action, to the incoming, or afferent, nerve impulses and the activity which they rouse in the gray matter of the cerebrum - Sensation.

Reading. - Wear and Tear, Mitchell. 


\section{CHAPTER IX.}

\section{THE SPECIAL SENSES.}

We have been considering the body as a collection of organs working together to serve the brain, the mechanism through which the mind operates.

We have especially studied the muscles as the only means by which the mind manifests itself to the outer world.

But how much mind would we have if we did not receive something from the outer world? Read the story of Kaspar Hauser. We are continually getting knowledge of the outer world and of the condition of our own bodies through the afferent nerves. We may never know fully what consciousness and thought are, but we can understand that to the brain are continually streaming nerve impulses that convey messages which the brain more or less completely interprets.

These incoming currents pass along myriads of nerve fibers. But the nerve fibers are all essentially alike. And the kinds of sensations that these currents arouse in the brain are but few. It is difficult to classify the senses, but it will serve our convenience to divide them into two groups.

General Sensations and Special Senses. - In distinction from the special senses, sight, hearing, etc., are the general sensations already referred to, such as hunger, thirst, fatigue, nausea, satiety, faintness, etc. They are often called "common sensations;" and Martin designates them as "sensations which we do not mentally attribute to the properties of external objects, but to the conditions of our own bodies." 
What We learn by touching Objects. - Let one person rest the hand flat on the table, palm upward, and close the eyes. An object placed on the palm may give rise to various sensations, so that it may be described as rough or smooth, light or heavy, hot or cold, wet or dry, etc. If the object is very heavy or very hot, it may cause pain. If now the thumb and fingers are raised and applied to the object, more definite information will be gained as to its shape, size, surface, etc. Now raise the object in the hand, and further appreciation will be gained as to its weight.

These experiments show that several sensations are involved in the handling of objects, and that the knowledge so gained is complex.

The sensations from the objects resting on the skin of the passive hand may, probably, all be referred to impressions made on nerve endings in the skin, and are called Cutaneous Sensations. They include: (1) the Pressure Sense, or Touch proper, (2) the Temperature Sense, and (3) Pain.

In lifting the object muscles are employed, and the sensation thus obtained is called the Muscular Sense.

Before taking up these sensations singly, let us review a few features of the nervous mechanism concerned.

Nerve Endings in the Skin. - We have learned that the skin consists of two layers, the epidermis and the dermis. We need now to recall those conical elevations of the dermis that we call papillæ. In these papillæ are certain important nerve endings. Since the sensory nerve impulses pass from without inward, it would be more appropriate to speak of the outer extremities of these nerves as their beginnings, or origins, rather than as "endings." Still, as they are peculiar, and are commonly termed endings, we may follow the ordinary usage.

There are several kinds of nerve endings in the skin and 
underneath it that receive the impressions which, carried to the brain, give $\mathrm{k}$ s sensations of touch (and allied sensations to be considered soon).

It would be out of place to try to go into this matter fully here. Some branching nerves end in the epidermis. In some of the papillæ are oral bodies in which nerve fibers end. Underneath the skin some nerve fibers end in little bodies shaped like a grain of wheat. Pressure on the skin affects these nerve endings, and starts impulses that pass along the sensor fibers to some nerve center,

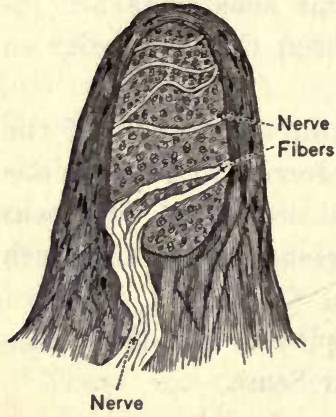

Fig. 86. Papilla of Skin with Touch Corpuscle. probably in the spina! cord, spinal bulb, or brain.

These "Touch corpuscles" are not regarded as essential for producing the sensation of touch, but some nerve endings in the skin do seem necessary; for if a nerve fiber be touched, not at the end, but somewhere along its course, we get, not a sensation of touch, but a sensation of pain. Except in the mouth and nose, we get little, if any, sense of touch from any organ but the skin. The lining of the digestive tube and the intermal organs generally are devoid of this sense. Compare Fig. 86 with Figs. 50 and 51.

The Sense of Touch. - Of the special senses the most general is that of Touch. Seeing and hearing, taste and smell, belong to very limited parts of the outside of the body, but we have the power of feeling all over the surface of the body.

Not only is the sense of touch the most general in being distributed over the whole of the body, but it is the most widely distributed sense throughout the animal kingdom. As 
we descend the animal scale we find many of the lower animals lacking some of the senses that we possess. But here we must be careful in our statements. Because an earthworm, for instance, lacks eyes, we may not say that it lacks sight. That is, while there is pretty good evidence that it cannot see objects distinctly as we can, it is not therefore necessarily unable to distinguish between light and darkness. Many of the lower animals lacking eyes show that they discern light. In many of the simpler forms of animal life there is no evidence of a sense of hearing; and it is extremely likely that if they have taste and smell, these senses are in a very rudimentary state of development. But in all these forms it is believed that "feeling" exists. Contact of their exterior with foreign objects is so often immediately followed by action that little doubt remains about their having the sense of touch. Even ameba may have, in a rudimentary state, the power to distinguish light, to taste, to hear. Still we have little or no evidence on these points, while we are pretty sure that it feels.

Further, the organs of special sense, and the nervous system generally, may be said to originate in the skin, and to be modifications of the sense of touch. It has long been known that the brain and spinal cord originate as an infolding of the skin, which afterward becomes completely shut off from the outer surface. "In general, it may be said that a study of the facts of development shows us that nerve-cells appear at first upon the surface of the body, but that during the growth of the organism the cells become shut off from the surface; and in order to maintain their connection with the periphery, long processes called nerves pass from the cells thus deeply imbedded to the surface."

The Pressure Sense. - The sense of touch, proper, is strictly a Pressure Sense. If we test the skin to find what 
regions are able to detect the least pressure, it is found that the forehead is most sensitive, and nearly equally so are the temples, back of the hand, and forearm.

The ability to detect differences of pressure is tested by finding what is the least addition to a weight required to make it seem heavier. For instance, if a weight of $\mathbf{1 1}$ grains is just perceptibly heavier than one of 10 grains, it does not follow that 1 grain added to a weight of 100 grains will give any palpable increase. To 100 grains must be added 10 grains before additional pressure is felt; that is, whatever the weight, there must be the same ratio of increase to increase the sensation. This is part of the law, already stated, of the relation of stimulus and sensation. The law is true only in a general way, and will not apply in extreme cases. It is stated that the forehead, the lips, and temples appreciate an increase of one-fortieth to one-thirtieth of the weight estimated, while the skin of the hand, the fingers, and the forearm require an increase of one-twentieth to one-tenth for its perception.

Discrimination of difference of pressure is more keen if weights are applied in rapid succession on the same spot than when applied at different places or after an interval of time. The weights used in these tests should press on equal areas of the skin.

The lingering effect of pressure, or After-Pressure, may be noticed after taking off a tight hat, skate-strap, shoe, or glove.

The Localization of Touch-Sensations. - We can usually locate very accurately any skin sensation. "If a point of the skin is touched, certain tactile corpuscles are irritated; these, in turn, set up impulses in sensory nerve fibers, and these impulses are carried by the fibers, first to the spinal cord, and then to the brain, where the fibers end in ganglionic masses in the gray matter of the cerebral cortex. There are 
thus projected, as it were, on the cortex of the brain, tactile centers for the hind leg, fore leg, neck, eye, ear, trunk, etc.; and it follows that each point of the skin has a corresponding point in the cerebral cortex. Thus for each stimulation of a point of the cerebral cortex there is a Local Sign, and thus we localize tactile impressions." The accuracy varies, and is ordinarily keenest where the nerves are most numerous. Where the sense of locality seems to be improved by cultivation, this appears to be due to keener discrimination in the brain-cells, and not to changes in the nerves or nerve endings. This is indicated in the fact that if the fingers of one hand become more discriminating by practice, it will be found that the fingers of the other hand, without special training, are also improved.

The delicacy of localizing touch is usually tested in this way. The blunted points of a light pair of compasses are allowed to rest gently on the skin of various parts of the body. If the two points are very close together they will be felt as one pressure. That part which can best distinguish, as two points of touch, these blunt points, is considered the most sensitive. By this test the tip of the tongue is the most sensitive, being able to distinguish, as two separate points of contact, the tips of the compasses when only one twenty-fifth part of an inch apart. Following is the order of degrees of sensitiveness : tip of tongue, tips of fingers, lip, tip of nose, eyelid, cheek, forehead, knee, neck; while the middle of the back seems least sensitive, the two points not producing two distinct sensations until they are more than two and a half inches apart. In general those parts which are most used, and those parts which are more freely movable, are most sensitive; for instance, the knee is much more sensitive than the middle of the thigh or the middle of the leg, and the elbow than the middle of the arm or forearm. If the compass 
points, about half an inch apart, be passed from the palm to the tips of the fingers, it will at first seem one line gradually separating into two diverging ones, owing to the keener localizing power as the finger-tips are approached.

"The reference of the sensations aroused by the excitement of definite nerve-cells to definite parts of the periphery is a power acquired through the physiological experiences of the earliest months of life. Through the sense of sight the seat of irritation is recognized, and through muscular sensation its relation to surrounding parts is experimentally explored, so that cumulative harmonious experiences of tactile, visual, and muscular sensations finally bring into correspondence the various areas with definite varieties of touch sensation." SEWALL.

The skin is more sensitive when moist; the barber therefore moistens the ball of his thumb before feeling the edge of the razor to see if it is sharp enough.

Illusions of Touch. - Aristotle's experiment has already been mentioned. If the "funny bone," or "crazy bone," be hit, i.e., if the ulnar nerve be bruised against the bone, sharp pain may be felt in the wrist and hand, and soreness of these parts may be felt for days, though they are not in the least injured, but only the nerve at the elbow. The currents along this nerve rouse sensation that we have learned to localize at the endings of the nerve fibers. So, too, after amputation of a hand or foot, there may for years be sensations referred to the missing member, probably due to irritation of the nerves of the stump. There is, then, no certainty of getting rid of a corn by amputating a toe.

The Temperature Sense. - Many cases are on record in which, from accident or disease, the pressure sense was lost and the temperature sense retained, or vice versa. Such facts have led to the belief that the temperature sense is distinct 
from that of touch, and has its own nerve fibers and nerve endings.

Since heat and cold are only differences in the degree of heat, we would expect both of these kinds of impressions to be received through one set of nerves. There seems, however, to be good evidence of two sets of nerve fibers, one for heat and the other for cold. In the common experience of the foot "going to sleep" by pressure on the sciatic nerve, or the arm from compression of the brachial nerve, the skin may be found, at a certain stage, to be only slightly sensitive to warmth, while distinctly sensitive to cold. In some diseases of the spinal cord the skin may be affected by warmth but not by cold. The sensations of cold and pressure seem to be usually lost or retained together, while those of warmth and pain have a similar connection. But more accurate results are obtained by touching the skin with a blunt metal pencil, warmed or cooled. If this be applied at regular close intervals it is found that some places feel the warm point, while others feel the cold. In this way the skin may be mapped out into "warm spots" (warmth-perceiving spots), and "cold spots" (cold-perceiving spots), and still other areas seem not sensitive to temperature. Heat or cold, if applied directly to a nerve trunk, does not rouse sensations. of temperature, but, if strong enough, produces pain. If the elbow be dipped into water at the freezing-point, a sensation not of cold but of pain is caused, and is felt in the hand. Heat and cold are not felt in the digestive tube except at or near the openings. If very hot liquid be swallowed, it may cause pain in the gullet and stomach. If a considerable quantity of warm liquid be taken, it may give a feeling of warmth from its effect on the skin of the abdomen, by conduction of heat outward. As with other senses, a sudden change in the degree of the stimulus is more certain to rouse sensation than a gradual change. 
The Muscular Sense. - As an example, we will take the case of estimating the weight of an object by holding it in the hand. Our estimate is thought by some to be the result of (1) direct consciousness of the degree of effort put forth; but probably it is (2) a sensation, or complex of sensations, aroused by nerve impulses from the organs used. There are afferent nerve fibers with endings in (1) the skin, (2) the muscles and tendons, (3) the joints. In extending the arm and moving it up and down, all three of these sets of nerve endings are probably stimulated, and impulses thence conveyed to the brain.

It is matter of doubt whether or not the impulses from the muscles are predominant, and consequently, whether the term "muscular sense" is the most appropriate. Peculiar nerve endings have been found in the tendons, and the joints are believed to have an especially rich nerve supply. It is not necessary that we actively use the muscles to have sensations of this kind. In passive moments, as the raising of the arm by another person, we have a "sense of position" of the parts, a considerable share of which is probably due to the tension of the skin and changes in the joints. There is, of course, some tension of the muscle, even in this passive movement, that might affect nerve endings in it. The muscular sense is closely related to the general sensibility already mentioned, if not a modified form of it.

It is difficult to realize the importance of this sense in our daily experience. We probably underestimate it, and attribute to sight too much of our knowledge of the external world. The fundamental facts concerning the objects about us are not obtained through sight alone. Such knowledge is based on complex judgments concerning the meaning of auditory and visual phenomena, according as they have, in past experience, been interpreted by tactile and muscular perceptions. 
That is, when reduced to its simplest terms, our most practical and important knowledge of the world is the outgrowth of tactile and muscular perceptions ; by and with them all other sense-perceptions have been corrected and compared.

"The fundamental education concerning the outer world, which engages the earliest years of every child, consists in accumulating and systematizing with other sense-perceptions tactile and muscular impressions of objects. A sensation is no sooner felt than some muscular movement, involving a definite muscular feeling, is made by which the character of the sensation is changed and experimentally tested under different conditions. The physiological process involved in building up sense-knowledge, therefore, embraces in alternation sensation excited by external objects, motion accompanied by muscular sensation, and change in original sensation. In other words, the motor and sensory impulses form a sort of balance, and both are necessary.

"When we consider that it is through muscular sensation that we derive our most accurate conceptions of the form, weight, and position of objects, and through which we explore our own body surface, and dịtinguish its areas of localization; that this is the fundamental sense by which the sensations arising in most other organs are tested and verified, and that it is from the sense of muscular movement that we can form ideas of time and space, it may well be regarded as the mother of all sense-perceptions. Normal muscles, even when functionally inactive, are still in a state of tonic contraction; it is not improbable that this tone is a reflex action whose sensory element is formed by the impulses traveling along nerves of muscular sensation. Such impulses are probably indispensable to the preservation of the equilibrium of the body." - Sewall.

An illustration of the assistance which touch and the mus- 
cular sense give to the sense of sight is furnished in the case of a boy who had been blind from birth, and received sight at the age of twelve years by means of a surgical operation. At first he could not distinguish a globe from a circular card of the same color until he had touched them. He knew the peculiar features of the dog and the cat by feeling, but not by sight. Happening one day to pick up the cat, he recognized for the first time the connection between the new serise of sight and the old familiar ones of touch and the muscular sense. On putting the cat down he said, "So, puss, I shall know you next time."

Pain. - When a heavy weight is laid on the hand it may cause pain. It would at first seem that the ordinary pressure sense, when unduly exaggerated, becomes pain. But there seem good reasons for considering pain as a distinct sense from that of touch intensified. It is thought that there are, throughout all parts of the body, nerves of "common sensibility" or "general sensibility," which keep the nerve centers informed as to the condition of all the various tissues, and that ordinarily we have no sensation resulting from the impulses; to use the language of the psychologist, "they do not rise above the threshold of consciousness." They may have some influence in adjusting the action of the different parts. We have seen how the blood flow to any part is continually adjusted without our knowing anything about it. But we are usually more or less conscious of the general condition of the body. We call by the name of "common sensations" such feelings as hunger, thirst, nausea, fatigue, depression, melancholy, restlessness, such as many experience preceding a thunderstorm, the feeling of general discomfort known as malaise, and its opposite, the feeling of general well-being. The body seems to have a set of nerves to give information as to the state of nutrition of the body, and as to its condition 
generally. These nerves, when the system is disordered in any part, may bring messages that cause intense pain. Of course, they are warnings (they are more than mere warnings; probably if the earlier indications of simple discomfort had been heeded, the later more emphatic messages of pain would not have been necessary). These messages of pain demand attention.

In reference to pain in the skin, it is held that the skin, too, has its nerves of general sensibility, and that these are distinct from those of touch and temperature sense. That when they are unduly stimulated they give rise to painful sensations. It is to be noted that the internal organs are ordinarily devoid of feeling, and that the skin is especially sensitive. The skin senses stand guard at the outposts, so to speak, of the body's camp, and give warning of approaching danger. No enemy may enter without being discovered by these keen sentinels, and the alarm is given. If it is not heeded, great harm may follow. And it is a comfort to know that the more severe wounds do not cause pain in proportion to their extent. When a person says his "lungs are sore" the pain is usually in the muscles of the chest from coughing. While there may be acute pain from the lungs, as in pleurisy, there is often deep-seated lung disease without pain from the lungs themselves. The muscles of the chest and back may be strained by lifting, and the soreness is erroneously attributed to the lungs or kidneys. Hence there is frequently a wholly needless apprehension of deep-seated disorder, whereas in reality there is merely a strain of superficial muscles. In amputating a limb the chief pain is in cutting through the skin. Some excellent authorities still hold the view that pain is merely the result of intensifying any of the simple sensations; but it is generally held that it results from the excessive stimulation of the nerves of general sensibility; as 
Foster puts it, "The constantly smoldering embers of common sensibility may be at any moment fanned into the flame of pain."

In the real "special senses," - sight, hearing, smell, taste, touch, and temperature sense, - we refer the sensation to some external object, whereas general sensations are subjective, referred to our bodies. Ordinarily we do not localize the common sensatious, and a farther indication of the relationship of pain and general sensation is in the lack of complete localization of pain. Slight pain, especially in the skin, may be closely located, but severe pain tends to become indefinite and diffuse. So we may class both the muscular sense and pain with the "general" rather than with the "special" senses.

Hunger and Thirst. - The cause of these sensations in a healthy body is plainly the need of food and water throughout the system generally. The sensation of thirst manifests itself in the throat, and the longing may be temporarily relieved by merely moistening the throat. So hunger may, for the time, be appeased by filling the stomach with indigestible material. But the sensation soon returns. The system has a crying need, and it is not to be put off by any such frauds. That these sensations are really demands made by the body as a whole may be shown by the fact that they are permanently relieved by introducing food and water into the body (by the rectum, for instance), in which case the throat and stomach have nothing given them directly. Since, however, food and drink naturally enter by the throat and stomach, the mucous membrane of these organs has become spokesman of the body for its demands.

The Sense of Taste. - We have seen that the tongue is a very muscular organ, and that when we are eating it helps to keep the food between the teeth, and that it does the chief part of the work in beginning the process of swallowing. 
We have also seen that the tip of the tongue has a very keen sense of touch. By means of its mobility and sensitiveness the tongue not only aids in mastication, but also cleanses the teeth afterward by detecting and removing small particles of food from the teeth.

The sense of taste helps us in judging of the frtness of anything that presents itself as a candidate for election as food. By reflex action the taste of agreeable substances aids in digestion by stimulating the glands, especially the salivary glands.

The surface of the tongue is covered with Papillæ. These are of three kinds. Most numerous are the Filiform papillæ,

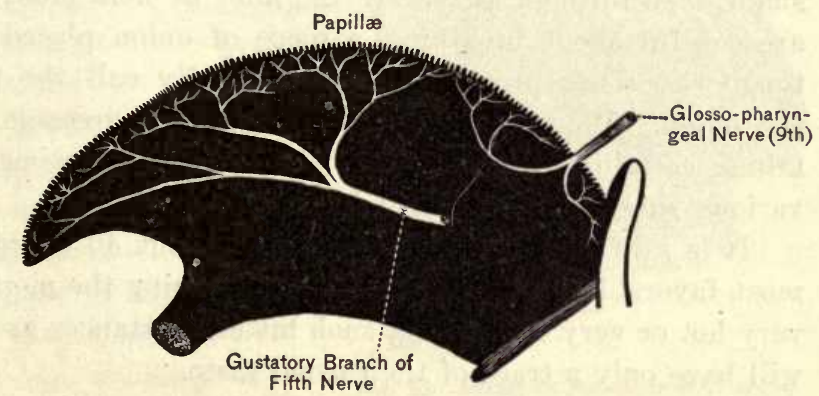

Fig. 87. Diagram of Tongue, showing Nerves and Papilloe.

slender, cylindrical projections. Like the papillæ of the skin, they seem to be organs of touch. Scattered among the filiform papillæ are small, bright red spots, which on examination are found to be shaped somewhat like a mushroom, the Fungiform papillæ. Near the base of the tongue are about a dozen larger papillæ, arranged like a letter $\mathrm{V}$ with its apex toward the base of the tongue. These are the Circumvallate papillæ, each having around it a deep circular furrow.

On the sides of this furrow are small oval bodies called 
"taste buds," connected with the ends of the nerves of taste. The nerves of taste are the Glosso-pharyngeal or ninth cranial nerves, distributed to the back part of the tongue, and a branch of the fifth pair of nerves, the Gustatory, to the front part.

Although we ordinarily speak of an article of food as "palatable," or "umpalatable," the sense of taste in the palate is only feebly developed.

Substances must be dissolved before they can be tasted. If the tongue be wiped dry and a few grains of salt or sugar be placed on it, the taste will not be perceived for a little time. Insoluble substances give no taste.

What we call Flavors affect us more through the sense of smell than through taste. If the nose be held shut, and we are careful about breathing, a piece of onion placed on the tongue does not produce what we usually call the taste of the onion. We may thus get rid of the disagreeable part of taking certain medicines. Let the student experiment with various substances as above indicated.

It is said that the temperature of about 40 degrees $F$. is most favorable for tasting; and after rinsing the mouth with very hot or very cold water, such bitter substances as quinine will have only a trace of their usual taste.

The tip of the tongue seems to be most sensitive to sweets and salines, the back part to bitters, and the sides to acids.

The Sense of Smell. - "The sense of odor gives us information as to the quality of food and drink, and more especially as to the quality of the air we breathe. Hence we find the organ placed at the opening of the respiratory passages and in close proximity to the organs devoted to taste. Taste is at the gateway of the alimentary canal, just as smell is the sentinel of the respiratory tract; and just as taste, when combined with smell to give the sensation we call flavor, influences the digestive process, and is influenced by it, so smell influ- 
ences the respiratory process. The presence of odors influences both the amplitude and the number of the respiratory movements. Thus the smell of wintergreen notably increases the respiratory work, next comes ylang-ylang, and last rosemary. The breathing of a fine odor is therefore not only a . pleasure, but it increases the amplitude of the respiratory movements. Just as taste and flavor influence nutrition by affecting the digestive process, and as the sight of agreeable or beautiful objects and the hearing of melodious and harmonious sounds react on the body and help physiological well-being, so the odors of the country, or even those of the perfumer, play a beneficent rôle in the economy of life." - M'K E N D R I C K

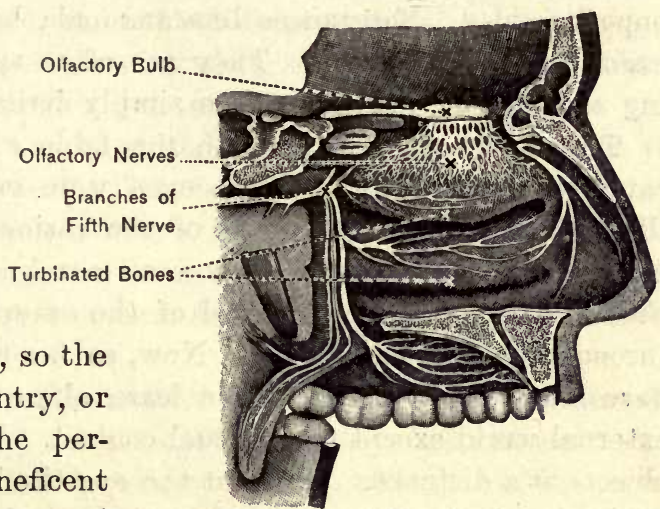

Fig. 88. Nerves of the Outer Wall of the Nasal Cavity. and SNodgrass.

In quiet breathing the air passes along the lower air passages just above the hard palate. The true olfactory passages are higher, but still in communication with this lower passage. When we wish to test the quality of the air, we sniff, that is, make a sudden inspiration by jerking the diaphragm down, and air from the outside then rushes into these upper nasal passages, over the walls of which the nerves of smell, the olfactory Nerves, are spread in the mucous membrane. The sudden rush of air against this membrare seems to aid 
greatly in getting the odor. The nerves have peculiar endings, and it is not known just how the substances produce their effect. The substances must be in a very finely divided state, probably gaseous. The mucous membrane is supplied with mucus, and the odorous substance, probably, is first dissolved in the mucus. The lower, or respiratory, passages have a more abundant blood supply, and are redder than the upper. In inflammation, owing to their narrowness, the passages, especially the upper, are often elosed by contact of the opposite sides. Substances like ammonia have no odor, but excite the tactile nerves. They are often spoken of as having a "pungent" odor, but are simply irritants.

The Sense of Sight. - In the fable of the blind man carrying the lame man whose eyes were good, we have an illustration of the dependence of the various organs on each other. We have considered how all our knowledge, both of the condition of our bodies and of the external world, comes through the nervous system. Now, so far as the senses we have studied are concerned, we learn almost nothing of the external world except from actual contact. But sight reveals objects at a distance. Without the eye the body is comparatively helpless. The lame man that the body carries is a slight burden in comparison with the assistance which he renders. We can' well afford to carry with us all the time two of these lame men to keep posted as to the objects beyond our reach. Of course touch is a great aid to our interpretations of what we see. But sight is evidently the main avenue of knowledge, the royal road along which come the messages which bring us the most news, which give us the keenest delight, which make us aware of most that we know of this world, and the only means of knowing that there are other worlds than the one we inhabit.

In order to understand, in any clearness, the action of the eye, we need to examine into its structure. 
A model of the eye should also be used in connection with the dissection, but it alone will not make the structure clear.

The Muscles of the Eyeball. - With bone-forceps or a strong knife cut away the bone at the outer angle of the eyesocket of the rabbit (almost any mammal will serve for this, though the bone is so thick in the calf or sheep that it will be difficult work without the aid of a good pair of bone-forceps).

1. With scissors trim away the white membrane around the front of the white of the eye; this was continuous with the lining of the eyelid, and is the Conjunctiva.

2. Find a muscle running along the roof of the eye-socket, which passes through a loop of tendon, near the edge of the orbit, and turns backward and outward to its attachment to the top of the eyeball. This is the Superior Oblique Muscle.

3. Beneath the eye find a muscle having its origin in the inner front part of the socket, and passing outward, to be inserted in the lower surface of the eyeball. This is the Inferior Oblique Musele.

4. Four straight muscles, the Superior, Inferior, Internal, and External Recti, are attached to the top, bottom, and sides of the eyeball ; find the origin of these, with that of the superior oblique, at the posterior extremity of the eye-socket.

5. Dissect away these muscles with any fat or other tissue, and find, extending from the back of the eye, the cylindrical Optic Nerve.

External Parts of the Eye. - The eye of the rabbit may be used, but that of the ox is better.

1. Observe the clear front part of the eye, the Cornea. Note its shape. Its wider end was at the inner angle of the eyelids.

2. Around the cornea find a whitish membrane, the Conjunctiva, which, a short distance back from the cornea, separates from the eyeball to turn forward and line the eyelid. 
3. The severed muscles of the eyeball, a mass of fat which forms a cushion for the eye, and other tissue, should be trimmed away, leaving the 0ptic Nerve.

4. Place the eye in its natural position, and make drawings of it, as seen from the front and from one side, naming the parts.

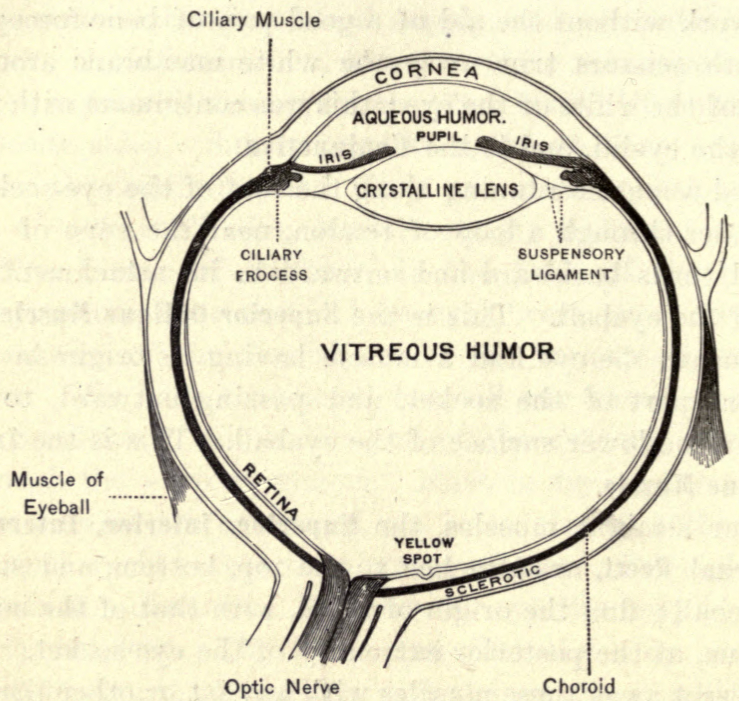

Fig. 89. Horizontal Section of Right Eye.

Dissection of the Eye. - Each member of the class should have an eye to dissect. To supply a large class it is best to send to a slaughtering-house in the nearest large city. If the eye muscles and other external parts have already been studied, it will not be necessary to remove the muscles and fat around the eye; in fact, they may well be left untouched, as they serve as a cushion to support the eye during dissection. The eye may conveniently be dissected on a 
small piece of board or shingle; and if it is desirable to turn the eye, it is better to do so by turning the support, as the eye usually sticks to the support, and the dissection may be injured by trying to move it.

Caution. - After the eye is opened be careful not to compress it. If the eye be held in the hand while trying to cut its tough outer coat, the jelly-like contents are easily squeezed out, ruining the dissection. Let the eye rest on the board all the time, and after first cutting through the cornea it is not necessary nor advisable to touch it with the fingers.

1. Lay the eye on the board, with the cornea uppermost. Hold the eye firmly with the thumb and fingers of one hand; with the thumb and forefinger of the other hand hold the blade of the scalpel half an inch from its tip; with a steady motion push the blade horizontally through the cornea, near its edge.

2. The liquid in the eavity back of the cornea is the Aqueous Humor.

3. Slightly enlarge the cut horizontally; then with the forceps take hold of the upper edge of the cut, and with the scissors cut around the margin of the cornea and remove it.

4. The dark membrane now exposed is the Iris. Pinch the eye slightly at the sides to make the iris show more distinctly. The hole in its center is the Pupil. With the forceps raise the edge of the iris around the margin of the pupil to see that it is here unattached to the structures underneath. Observe the color and markings of the iris.

5. From one end of the pupil cut outward to the outer margin of the iris ; then cut around its outer margin and remove it. Observe the color and markings of the posterior surface.

6. The body now laid bare is the Crystalline Lens. Touch it.

7. Lay a piece of newspaper close to the eye, on which to 
receive the lens, which sometimes pops out suddenly. With a sharp knife make a quick, light gash across the surface of the lens to cut through the thin coat which envelops it, the Lens Capsule. Usually the lens may be made to come out by applying gentle pressure to the sides of the eye with the thumb and finger. If not, enlarge the opening thus made, and carefully pry out the lens with the handle of the forceps, noting closely, in so doing, the difference between the front and back surfaces. Lay the lens on the piece of newspaper, and look through it at the letters. Make a drawing of the lens as seen from the front, and as seen from one side, naming the front and back surfaces.

8. In removing the strip of eye-coating, as directed below, be extremely careful not to drag the clear jelly-like vitreous humor. The parts must be lifted gently by the forceps, and if the clear jelly-like mass adheres, it must be cut through, horizontally, with the scissors. With the scissors now cut outward about one-half of an inch from the edge of the hole made in the front of the eye; then cut clear around the eye and remove a strip of this width, thus enlarging the opening before made. On the inside of the strip removed there may be found radiating black ridges, the Ciliary Processes.

9. Carefully pick away with the forceps, and snip away with the scissors, everything on the surface of the clear mass beneath.

10. The substance filling the remainder of the eye-cavity is the Vitreous Humor.

11. Through the vitreous humor the entrance of the optic nerve may be seen with the blood tubes radiating from it. If necessary, carry the dissecting board to a window to let the light enter from above.

12. The tough outer coat of the eye is the Sclerotic Coat.

13. Inside the sclerotic is the dark Choroid Coat. 
14. The inner, nearly transparent, pinkish or whitish coat is the Retina. At this stage of the dissection it has probably become slightly wrinkled, and the white ridges may be seen radiating from the entrance of the optic nerve.

Drag out the vitreous humor, and note the soft, whitish or pinkish retina; observe that it is a continuation of the optic nerve. Tear away the retina, noting its consistency. Note the color and luster of the inner surface of the choroid coat. The dark layer on the inside of the choroid coat is the pigment-layer (outer part) of the retina, which adheres to the choroid, and is torn loose from the rest of the retina.

The reflection of light from this surface of the choroid coat causes the color seen in the eyes of some animals. Turn the remaining coats inside out, and tear the choroid coat from the sclerotic. Observe the blood tubes passing from one to the other.

The Retina. - The chief thing in the eye is the retina. Without this all else is useless. If light of sufficient strength falls on the retina.it stimulates elements in the outer layer (rods and cones), and the nerve impulses, thus started, pass along the fibers of the optic nerve to the brain, and we have the sensation of light. But in order to see anything distinctly, the light must fall on the retina in such a way as to form a distinct image of that object. If the lens be removed, or becomes opaque, as in "cataract," we fail to see distinctly, though we may discern light from darkness. The other parts of the eye exist to form images on the retina. The cornea, lens, and the aqueous and vitreous humors are the parts directly concerned in forming the images. Light from an object passes through the cornea, aqueous humor, lens, and vitreous humor, and the rays are so refracted as to form an inverted image. If this image falls on a good retina we see well. If these parts do not all work well we see poorly. 
The retina is very complicated in its structure. No less than eight layers have been distinguished, as shown in Fig. 90. Of these layers the outermost, the layer of the rods and cones, is the one directly concerned in appreciating the differences in the vibrations of the light.

The rays of light pass through the retina, and produce their effect on the rods and cones which constitute the outer

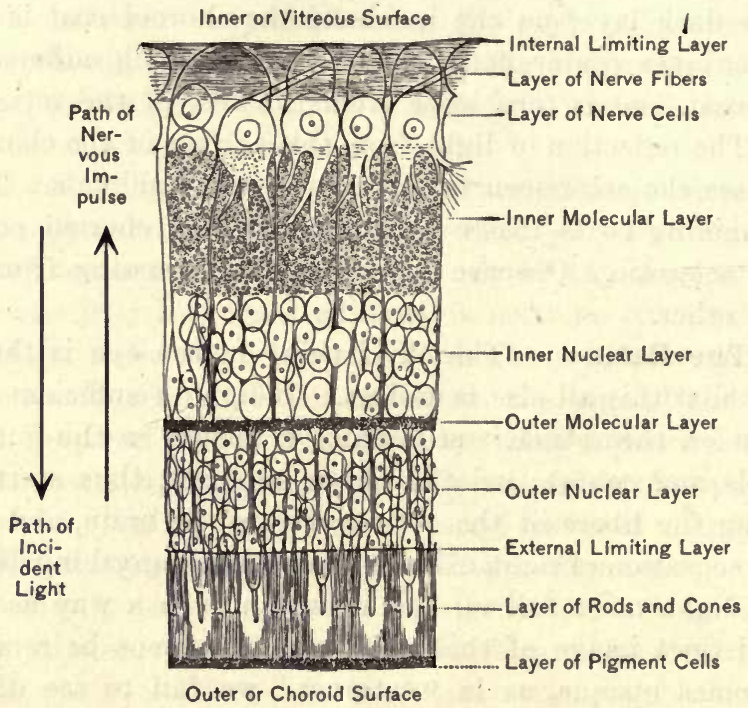

Fig. 90. Diagrammatic Section of the Human Retina.

(back) layer; and the nerve impulses aroused by the light must return through the thickness of the retina to be conveyed along the nerve fibers of the innermost layer of the retina to the optic nerve.

The Center of Distinct Vision. - Near the middle of the back part of the retina is the Yellow Spot (macula lutea), 
and in the center of this a slight hollow. In this region vision is most distinct, especially for color.

Protection of the Eye. - The outer coat holds all in place, and gives strength for attachment of the muscles that give motion to the eyeball. The Lacrymal Gland, or tear gland, is just above the outer angle of the eye, and pours its secretion over the eyeball in weeping, or when there is need of an unusual supply of tears. The lids serve as curtains to admit or shut out light, and, by winking, with their own secretion, a fluid mixture of salt water and mucus, to wash the eye. It is as though a man were kept all the time in front of a plate-glass window, with water and rubber scraper, to keep it clean and bright. The lacrymal secretion is, ordinarily, carried off as fast as it is made, by two ducts beginning at the inner angle of the eye, one on each lid; these two ducts soon unite, and empty by one outlet into the nasal cavity. If these ducts are stopped, or if the secretion be formed very rapidly, the liquid overflows on the face as tears. Then, too, the eyeball is set well in its bony socket to shield it from harm. The cornea, or window, is a continuation of the sclerotic coat.

The choroid coat is richly supplied with blood tubes and dark pigment to absorb light so it may not be reflected about in the eye. The choroid coat, continued forward, becomes the iris, but is incomplete, leaving the circular hole in this dark curtain. The pupil looks dark because we are looking into a dark room. A thin membrane, called the hyaloid membrane, lines the inner surface of the retina. Arising from the sclerotic coat at the outer border of the iris is a muscle, the ciliary muscle; it is inserted at the margin of the lens by means of fibrous strands that form an intimate part of the capsule inclosing it.

Take a single lens that is convex on both sides, such as in the common tripod lens, or any hand magnifier. Hold this up 
in front of a window, and eatch the inverted inage of the window on a piece of paper held back of the lens. 'This illustrates how the image of an external object is formed by the

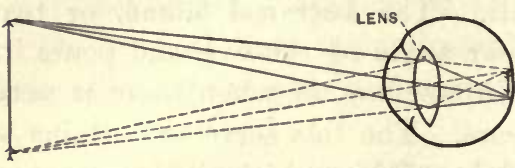

Fig. 91. The Formation of an image on the Retina. crystalline lens upon the retina of the eye. If two lenses of different thickness can be obtained, it will be seen that the thicker lens (if both have the same diameter) will make an image closer to the lens than the thinner one.

Adjustment of the Lens for Seeing at Different Distances. - If we look up from a book we are reading, we do not realize that any change is necessary in the eye for us to see a distant object. But it is easy to prove that we cannot, at the same time, see distinctly a near and a distant object.

Stick a pin at each end of a book cover. Hold the book at about the usual distance for reading, so that the two pins are in a line with the eye. Look closely at the nearer pin, and the second pin will appear indistinct. Now look closely at the head of the farther pin. The nearer one may be seen, but not sharply. Another way of testing this: hold the tip of a pencil in a line with any object, say a picture, on a wall opposite. In looking at the tip of the pencil the picture is dim. Now look by the pencil at the picture, and the point of the pencil will be blurred.

What changes does the eye make to enable it to see clearly, now a near, now a far object?

When the photographer places his camera, he moves the ground-glass plate back and forth till the image is distinctly formed on the plate. We cannot move the retina back and forth, so we change the shape of the lens. When we look at 
a near object the lens becomes thicker, and when we look at a distant object the lens becomes less thick. In looking at an object that is near, the ciliary muscle pulls on the hyaloid membrane, and draws it forward (since the muscle is fastened at the point where the iris joins the cornea). When the hyaloid membrane is pulled forward, the lens is released from pressure that was given it by the lens capsule. Now the lens becomes thicker because it is elastic, and when it is not subject to pressure it tends to become relatively thick. When we look at a distant object the muscle relaxes, and the capsule presses on the front of the lens and flattens it, so ad-

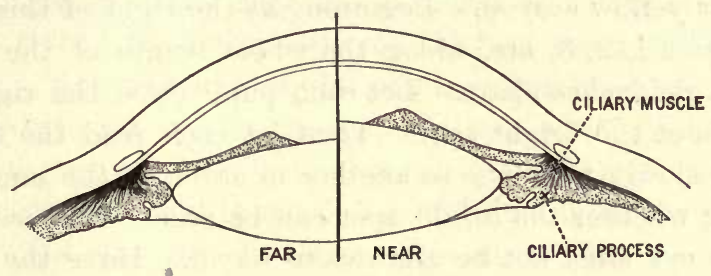

Fig. 92. A Diagram to Illustrate Accommodation.

justing for far sight. It should be noted that adjustment for near sight is brought about by muscular effort, hence is fatiguing; whereas adjustment for far sight is accomplished mechanically, without effort. In old age the lens usually becomes less elastic, and cannot adjust for near sight. Since it is unable to grow more convex, artificial lenses (eyeglasses) may be used to enable one to see near objects clearly. Most elderly people see fairly well at a distance, but use glasses for reading or any close work.

In "near-sighted" eyes, the eyeball is often too long from front to back, so the image is not thrown on the retina. Concave glasses remedy this defect. The eye may also be too 
short, and need special glasses. The refracting surfaces (cornea or lens) may be unequally curyed, causing astigmatism. For most of these defects the occulist will supply suitable glasses.

The Blind Spot. - The structure of the retina is exceedingly complicated, much more so than any of the other nerve endings. Light must fall on these special structures to have any effect. Falling on the optic nerve itself has no effect in giving a sensation of light. And if the light falls on the spot where the optic nerve enters the eyeball we see nothing. At the left (as looked at by the class) of a long blackboard make a bright circular spot, three inches in diameter, with white or yellow crayon. Beginning at the right of this, write the figures $1,2,3$, etc., along the whole length of the board, about eight inches apart. Let each pupil close the right eye and look at the bright spot. Then let each read the figures, passing slowly from one to another in order, at the same time noticing whether the bright spot can be seen. To succeed in this the eye must not be allowed to waver. Have the pupils tell when the bright spot disappears, then read on, and note when the spot reappears.

In the following experiment shut the right eye, and be careful not to let the left eye waver.

Read this line slowly. Can you see the star all the time? If the star does not disappear before reaching the end of the line, let the eye travel on across the right-hand page, or hold the book nearer the face. In the human eye the optic nerve enters the eye not in the center, but nearer the nose, so that in turning the left eye toward the right at the proper angle, the image of the star falls upon the spot where the optic nerve enters. As this spot is insensitive to light, the star no longer appears.

The optic nerve, while capable of carrying nerve impulses 
that cause sensations of light, is not itself sensitive to light. If the optic nerve be cut, it does not give pain, but gives the sensation of a flash of light.

While most of the fibers from each optic nerve cross to the other side of the brain, some fibers go to the same side of the brain. We can therefore better understand the close sympathy that we know exists between the two eyes. In many of the lower animals, a fish, for instance, the two optic nerves cross without any intermingling of fibers.

Pain, felt in the eyes, comes from impulses conveyed, not by the optic nerve, but by a branch of the fifth pair of nerves (the nerves of sensation for most of the face).

Regulation of the Amount of Light Admitted into the Eye. - Hold a hand-glass between the face and a well-lighted window. Note the size of the pupils. Quickly turn toward the darkest part of the room. We see, what we have all noticed in watching the eyes of a cat, that when subject to a bright light the pupil is small, but with less light the pupil is larger. The iris has circular muscle fibers that reduce the pupil when there is too much light for the eye, and when the light is feeble the pupil opens wider.

Color Sensations. - The difference in colors is due to the differences in the rapidity of the vibrations of the waves of light, as in sound differences in the rapidity of the vibrations of the sound waves cause the various degrees of pitch. Many interesting experiments may be made with color sensation, most of which are difficult of explanation. Fasten a bright red wafer or seal on a white card. Look intently at the center of the red spot till the eye is tired. Then quickly look at a point in the white surface. What color appears? This may be repeated with other colors.

Color Blindness. - It is found that some persons cannot distinguish certain colors. Blindness to red and green are 
most common. This is a matter of importance among railroad men and sailors, where it is necessary to distinguish red and green signals.

Stereoscopic Vision. - In looking at an object with one eye there is seen more to the side of that eye, while the other eye sees more of the other side, considerable of the object being seen with both eyes. The effects produced on the two eyes are united, and so we better see objects as solids. 'This is what is termed stereoscopic or Binocular Vision.

Duration of Impressions of Light. - Most boys have amused themselves around a bonfire by whirling a stick with a glowing coal on its end. The continuous circle of light thus

- produced indicates that the impression of light remains for a time, in this case until the stick completes the circle, giving a continuous line of light. By shaking the hand up and down rapidly the same effect may be produced; that is, we see the hand in all positions at the same time. Or when riding in a carriage the spokes of the wheels blur together because the impression of each lingers till another has taken its place. But if we shut the eyes quickly, we may keep distinct the impression of the last positions, and so see them distinct from each other. Better still, shut the eyes while looking at the wheel, then open and shut them as quickly as possible.

Again, if one looks at a bright lamp and then closes the eyes, there may remain the same appearance as when we looked at the object itself. This is called the Positive Afterimage. Or sometimes, especially after looking long at a bright light, we may, on closing the eyes or looking away, see a dark spot of the same shape as the bright one we looked at. This is called the Negative After-image.

The Care of the Eyes. - 1. In reading we wish light from the printed page. Hence we should avoid light entering the eye from any other source at this time. While reading, then, 
do not face a window, another light, a mirror, or white wall, if it can be avoided. In a room, white walls are likely to injure the eyes. Choose a dark color for a covering for a reading-table. Sewing against the background of a white apron has worked serious mischief. Direct sunshine very near the book or table is likely to do harm.

2. Preferably have the light from behind and above. Many authors say "from the left," or "over the left shoulder." In writing with the usual slant of the letters this may be desirable. But vertical writing is now strongly advocated, as it enables one to sit erect, and have the light from above and equally to the two eyes. Having stronger light for one eye than for the other is bad. Sitting under and a little forward of a hanging lamp will thus give the light equally to the two eyes and send no light direct into the face. In reading by daylight avoid cross-lights so far as possible. The incandescent electric light has an advantage in being readily lighted, without matches, and in giving out little heat, thus making it valuable for house-lighting; but owing to its irregular illumination (due to the shadow cast by the wire or filament), it is not well suited for study or other near work. For this purpose an Argand gas or kerosene burner is much to be preferred, since it throws a soft, uniform, and agreeable light upon the work.

3. Reading out-of-doors is likely to injure the eyes, especially when lying down. To try to read while lying in a hammock is bad in many ways. Too much light directly enters the eye, and often too little falls upon the printed page.

4. Do not hold the book or work nearer the eyes than is necessary. So far as possible avoid continuous reading in large or heavy books by artificial light. Such books being hard to hold, the elbows gradually settle down against the sides of the body, and thus, without thinking about it, the book 
is held too close to the eyes, or at a bad angle, or the body assumes a bad position.

5. Frequently rest the eyes by looking up and away from the work, especially at some distant object. One may rest the eyes while thinking over each page or paragraph, and thus really gain time instead of losing it.

6. Have light that is strong enough. Remember that the law of the intensity of light as affected by distance is that at twice the distance from the source of light the light is only one-fourth as strong. Reading just before sunset is risky. One is often tempted to go on, not noticing the gradual diminution of light.

7. Save the easiest reading for the evening. Newspapers, as a rule, have neither good print nor good paper. If the eyes have much work to do, finish this kind of reading by daylight if possible, and by artificial light read books, which usually have better type and better paper. In all ways endeavor to favor the eyes by doing the most difficult reading by daylight, and saving the better print and the books that are easier to hold for work by artificial light. Writing is usually much more trying to the eyes than reading. By carefully planning his work the student may economize eyesight, and it is desirable that persons blessed with good eyes should be careful, as well as those who have a natural weakness in the eyes; for it often results that those inheriting weak organs, by taking proper care, may outlast and do more and better work than those naturally stronger, but who through carelessness injure organs by improper use or wrong use (ab-use).

8. Reading before breakfast by artificial light is usually bad.

9. Many eyes are ruined during convalescence. At this time the whole system is often weak, including the eyes. 
Still, there is a strong temptation to read, perhaps to while away the time, perhaps to make up for lost time in school work. This is a time when a friend may show his friendship by reading aloud to the convalescent.

10. If one finds himself rubbing his eyes, it is a clear sign that they are irritated. It may be time to stop reading. At any rate, one should find the cause, and not proceed with the work unless the irritation ceases. If any foreign object gets into the eye, as a cinder, it is better not to rub the eye, but to draw the lid away from the eyeball and wink repeatedly; the increased flow of tears may dissolve and wash the matter out. If you must rub, rub the other eye. If it be a sharp-cornered cinder, rubbing may merely serve to fix it more firmly in the cornea or the mucous membrane of the inner surface of the eyelid. If it does not soon come out, the lid may be rolled up over a pencil, taking hold of the lashes or the edge of the lid. The point of a blunt lead pencil is a convenient and safe instrument with which to remove the particle. Sometimes being out in the wind (especially if unused to it), together with bright sunlight, may irritate the eyes. If after such exposure one finds lamplight irritating, he will do well to go to bed early, or remain in a dark room.

11. Be careful to keep the eyes clean. Do not rub the eyes with the fingers. Aside from consideration of rules of etiquette, there is danger of introducing foreign matter that may be very harmful. It is very desirable that each person have his individual face towel. By not observing this rule, certain contagious diseases of the eyes often spread rapidly.

12. If there is any continuous trouble with the eyes, consult a reliable oculist. Many persons injure the eyes by not wearing suitable glasses. On the other hand, do not buy glasses of peddlers nor of any but reliable specialists. One 
may ruin the eyes by wearing glasses when they are not needed. Sight is priceless.

The Sense of Hearing. - The ear passages are inclosed by the hard bones of the head. The ear is, in consequence, difficult to dissect. It is very desirable to have a model of the ear, and a good model may be bought for less than ten dollars. The ear may be dissected in a cat or rabbit by fol-

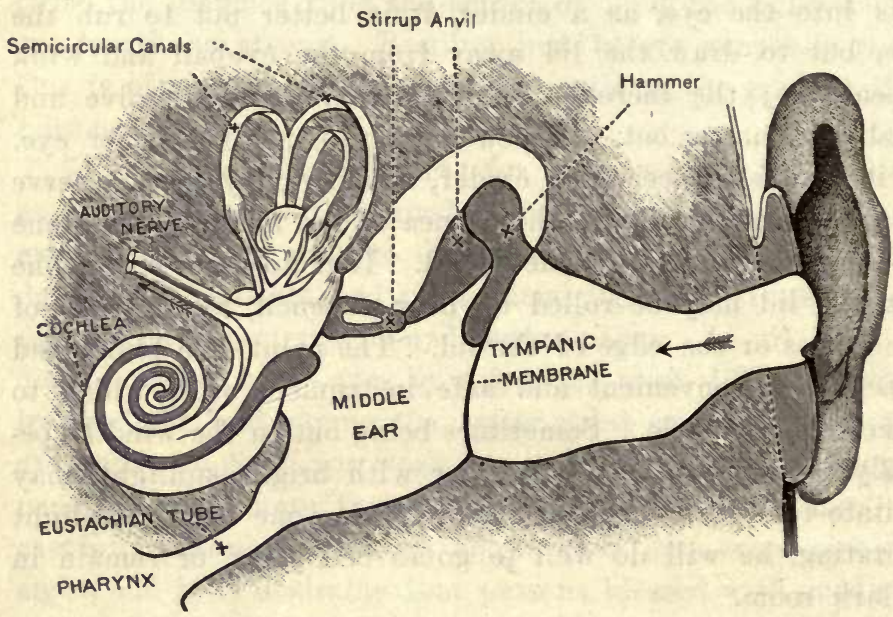

Fig. 93. Diagram of the Ear.

lowing the accompanying description. It will take time and patience to trace all the parts.

The ear is a much more complicated organ than would naturally be supposed. The parts of the ear are the External, Middle, and Internal ear. The external ear gathers the soundwaves and directs them into the opening of the ear, but the loss of the external ear does not seriously interfere with hearing. The hole leading inward from the ear extends a little 
more than an inch, and is then completely shut off from the cavities beyond by a thin membranous partition, the Tympanic Membrane or Drum-skin. The skin of the ear dips into and lines the external tube, and continues as a very thin layer over the membrane of the tympanum. The Auditory Meatus, as this passageway is called, is guarded by hairs, and is further protected by wax secreted by glands of the lining. Beyond the membrane of the tympanum is a cavity called the middle ear. Extending across the cavity of the middle ear is a chain of very small bones, the Hammer, Anvil, and Stirrup, the hammer being attached to the inner surface of the membrane of the tympanum, and the stirrup being fastened by its base to the wall of the internal ear. The middle ear communicates with the back part of the mouth (pharnyx) by means of a narrow tube called the Eustachian Tube. This tube is probably closed most of the time, but opens when we swallow.

The internal ear consists of several complicated cavities and tubes which contain a liquid in which rest the nerves. The principal cavity is the Cochlea, or snail-shell cavity, in which the nerve endings are connected with an exceedingly complicated apparatus.

Sound-waves set the drum-skin or nembrane of the tympanum in vibration; the vibrations are conveyed by the chain of bones across the middle ear to the liquid of the inner ear. Through the complicated apparatus of the snail-shell the vibrations of the liquid are made to start nerve impulses in the fibers of the auditory nerve, and when these nerve impulses are rightly received and interpreted by the brain, we have a sensation called Sound.

The Equilibrium Sense. - Probably most of the senses contribute to the maintaining of the equilibrium of the body by giving information as to position, motion, etc., especially sight and the muscular sense. 
Only that part of the auditory nerve which is distributed in the "snail-shell" of the ear is now supposed to have to do with hearing. It is no longer believed that the Semicircular Canals are concerned with the process of hearing. There seems to be good evidence that the semicircular canals inform us as to changes of the position of the body, and they are regarded as the seat of an "equilibrium sense." The fact that one of these canals is horizontal, and that the two vertical canals are at right angles to each other, strengthens this belief. It is thought that each of these canals detects movements in its own plane. The experiment has been made of placing a man on a table that turned easily; with the eyes shut the subject could usually detect fairly well the changes of position from rotation of the table. What is known on the subject comes partly from observation in cases where these parts are diseased (which, in itself, does not cause loss of hearing), and by operating on lower animals; in both of these lines of observation injury to these parts appears to be followed by dizziness, loss of power to maintain equilibrium, etc.

The Care of the Ear. - In cleaning the ear no hard substance should be used; even the finger-nail is likely to do harm. A moistened cloth should be used. If this is not sufficient, a physician should be consulted.

In washing the ear it should be thoroughly dried before being exposed to a wind, especially a cold wind. The rapid evaporation may cool the parts so rapidly as to cause trouble. It is not well to stuff the ears with cotton. If there is any trouble with the hearing, of course a physician should be consulted without delay.

The existence of an organ of hearing implies the existence of what? Why have we these organs of hearing? Is it merely a means of protection? Is it that we may enjoy the music of nature, such as the songs of birds? Is there not 
one sound that makes sweeter music than the most gifted of feathered songsters, surpassing all the instruments of man's device, even the violin, with its almost human flexibility and range of expression?

What sound communicates to us the most of thought and sympathy?

What sound was it Robinson Crusoe, in his dreary solitude, most longed to hear?

Reading. - Physiology of the Special Senses, M'Kendrick and Snodgrass; The Senses of Man, Bernstein. 


\section{CHAPTER X:}

\section{THE VOICE.}

THE delicate mechanism and capabilities of the ear are fully matched by the fine adjustment and range of the voice. The organ of the voice is well worthy of study, if we look at it merely as a most ingenious contrivance, to say nothing of its importance to us as a means of expressing thought.

But to understand the mechanism, it is necessary to examine the structure itself, for no mere description can make clear its parts and their relations.

We can learn a little from the observation of our own mouths and throats. The projection of the throat known as "Adam's Apple" is one angle of the Thyroid cartilage. A ridge may be felt running downward from the projecting angle. Above the Adam's apple a depression may be felt. Press the tip of the finger lightly into this depression and perform the act of swallowing. It will be noted that the Adam's apple is drawn upward and closer to the bone above the depression. This bone is the Hyoid bone; it is connected with the larynx below the base of the tongue. Below the thyroid cartilage another cartilage may be felt, the Cricoid cartilage. Below this is the windpipe with its rings of cartilage. The general form of the whole larynx may be felt in a person not overburdened with fat.

By depressing the tongue and looking into the mouth the tip of the epiglottis may possibly be seen at the base of the tongue. Beyond these points we cannot learn much without dissection. A small mirror set obliquely on a handle (like 
those used by dentists) may be inserted through the mouth so that the larynx can be seen from above. But the meaning of what would be thus seen would not be very clear without a careful dissection of the larynx.

The Larynx of the Calf. - 1. The front of the larynx is readily distinguished by the projection of cartilage known as the Adam's Apple.

2. Along the back of the larynx runs a thick muscular tube, the Gullet, with a whitish lining, the mucous membrane.

3. Trim away the muscles and other tissues from the front and sides of the larynx. The large cartilage forming the greater part of the front of the larynx is the Thyroid Cartilage.

4. Observe the band of muscles attached to either side of the thyroid cartilage and passing horizontally back around the esophagus.

Cut away this muscle as completely as possible, and entirely remove the gullet. Note that the whitish or yellowish Mucous Membrane which lines the gullet is continuous with the lining of the larynx. Study now more fully the shape of the thyroid cartilage.

5. Back of the upper part of the thyroid cartilage, covering the upper end of the larynx, is the arched Epiglottis. Feel of it to learn its consistency. Press it upward and forward, then downward and backward; observe that it now covers the entrance to the larynx; note the position it takes when released.

-6. Just back of the upper angle of the thyroid cartilage find a muscle connected with the base of the epiglottis; pull this muscle to determine what effect its shortening produces on the epiglottis.

7. Under the thyroid cartilage in front observe a narrow ring of cartilage not much wider than one of the rings of the 
trachea. Move this up and down to prove that it is distinct from the thyroid. This is the Cricoid Cartilage.

8. Observe the sheet of muscle passing from the cricoid to the thyroid. Again move the cricoid toward and from the thyroid; what does this muscle do? Cut away this muscle from one side, and see that the cricoid cartilage widens as it passes backward. How are the cricoid and thyroid hinged together?

9. Projecting upward and backward from the top of the larynx are two curved yellowish cartilages, the Arytenoid Car. tilages. Move them about to see that they are movable, and that they rest on the upper edge of the back part of the cricoid cartilage.

10. Move the arytenoid cartilages backward and forward, meanwhile watching the inside of the larnyx from its lower opening. The projecting ridges, which meet just back of the Adam's apple, are the Vocal Cords. What effect is produced on the vocal cords by the movements of the arytenoid cartilages ?

11. Observe the connection of the thyroid cartilage with the cricoid by means of a downward projection of the former. Cut away, all of this half of the thyroid cartilage. Notice the slender Hyoid Bone loosely connected with, the upper horn of the thyroid.

12. Examine now the muscles which move the arytenoid cartilages.

(a) On each side of the posterior surface of the cricoid is a muscle passing upward to be attached to the corresponding arytenoid; this is the Posterior Crico-arytenoid Muscle. Dissect it loose from the cricoid at its origin below. By pulling, determine its action on the arytenoid, and through the arytenoid on the vocal cord.

(b) Arising from the upper edge of the side of the cricoid 
cartilage, and passing upward and backward to the arytenoid, is the Lateral Crico-arytenoid Muscle; cut away at its origin close to the cricoid, and demonstrate its action on the arytenoid cartilage and vocal cord.

(c) A broad muscle arising along the whole length of the angle of the thyroid, whose fibers converge to the arytenoid cartilage. This is the Thyro-arytenoid Muscle; cut it across near its origin, dissect it loose, and by pulling it toward its origin prove its action.

(d) On the posterior surface of the arytenoids is the small Arytenoid Muscle.

13. Cut between the arytenoid cartilages and remove one of them. Examine the joint between the arytenoid and cricoid. Note the synovia lubricating the joint.

Trim away the muscle from the arytenoid cartilage and study its shape more fully. Fit it again to its place, and recall the motions given by each muscle.

14. Now examine the arytenoid cartilage and the vocal cord of the opposite side; move the arytenoid back and forth, watching the rocal cord.

15. Remove the epiglottis, and cut into it to see its structure.

16. Dissect away the parts of the other side from the inside, reviewing the above points.

By examining the larynx we see that its cartilages are connected with each other by ligaments which allow considerable freedom of motion; that they are also connected by muscles which move them one upon another. The range of movement of the arytenoid cartilages and the muscular control of these cartilages is of special interest, for we see that the vocal cords, so-called, are attached to these cartilages, and that the chief variations of the voice are thereby regulated.

The vocal cords are badly named. They are seen to be 
mere ridges projecting from the sides of the larynx. Under the covering of mucous membrane are ligaments and muscles that may be stretched to various degrees and placed in different positions, according to the sound that is to be produced.

While we are quietly breathing, the vocal cords, or bands, lie back, like low ridges, against the side of the larynx, and offer nearly the whole channel of the larynx for the free passage of air for breathing purposes. But when we wish to

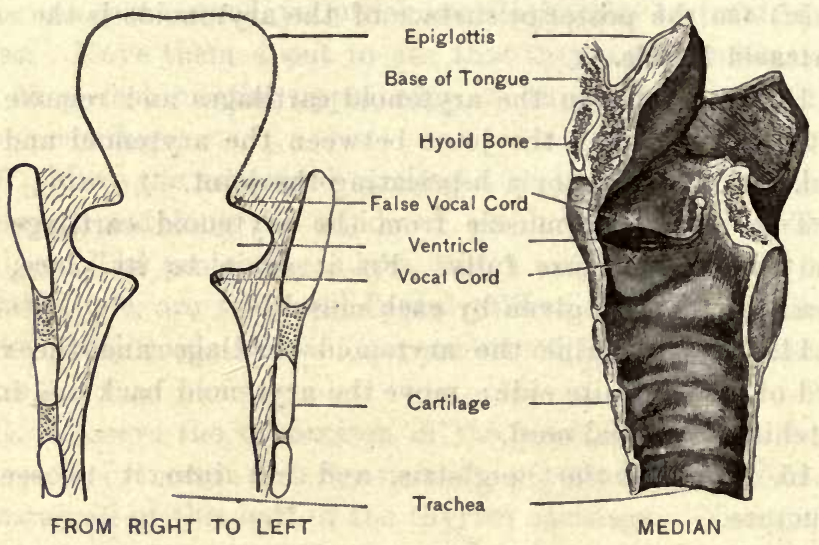

Fig. 94. Longitudinai Sections of the Larynx.

produce vocal sound, the vocal cords are made to stand out farther from the side walls, and interfere with the free passage of the air. In examining the larynx it is seen that the vocal cords are attached close to each other in front, but that at the back of the larynx they diverge widely (in the position of rest), forming a letter $\mathrm{V}$, with the angle of the $\mathrm{V}$ in front, just back of Adam's apple. "When changes in the voice or in breathing are being made, the white glistening vocal cords may be seen to come together or to go apart like the blades of 
a pair of scissors." In a high note the cords are close together and nearly parallel. As the air is forced past the approximated edges of the vocal cords, they are set in vibration, and produce the sound called the voice. The principle of the action of the vocal cords can be illustrated by the common toy known as the the squeaking balloon, or "squawker." Here the air is driven out past a band of rubber stretched across the inner end of the tube. If instead of one band with both edges free, we were to tie on the inner end of the tube two bands of rubber, each covering the outer edge of the tube,

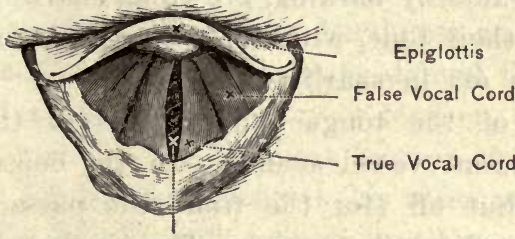

Glottis Narrowed, High Note

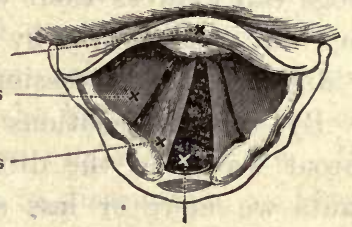

Glottis Wider, Quiet Breathing

Fig. 95. The Larynx, as Seen by Means of the Laryngoscope, in Different Conditions of the Glottis.

leaving the inner edge of the rubber free, and with the two bands touching at one end and considerably separated at the other end, we would have a pretty fair resemblance to the larynx. As in many musical instruments, the vibrations of the membrane itself alone would be too feeble to have much effect. In the violin, piano, drum, etc., the vibrations are reenforced by the vibration of a body of air contained within. So here the vibrations of the cords are reënforced and modified by the air spaces above. The loudness of the voice depends on the force with which the air is driven past the cords, together with the size and condition of the cords themselves. . Pitch depends on the rapidity of the vibrations, which is de- 
termined by the length of the cords and their tension. Other things being equal, the size of the larynx would determine the pitch.

The larynx by itself produces vocal sound merely. In speech the sounds produced in the larynx are much modified by the lips, tongue, teeth, cheeks, etc.

We have voice as soon as born, but we only gradually acquire the power of speech. Mammals, birds, and some of the lower vertebrates have voices, but they have not speech. This distinguishes man from the animals below him, though perhaps some of the higher apes have it in a slight degree. Dogs can express their wants by barking, growling, snarling, etc., but it is mostly by their tone, with their attitudes, and a slight facial expression (as in snarling).

By various positions of the tongue and organs of the throat we make the different vowel sounds. In the consonants we more or less shut off (for the time) the passage of air, and so stop, or modify, the sound. This is hardly the place to study and analyze the sounds of our spoken language, yet it may be found profitable to watch the different organs as each sound is produced; for when the structure and relation of the different parts concerned in the production of these sounds are better known, the definitions and statements of the books will be much more fully understood.

Since no two throats are exactly alike, no two voices sound just the same. The size and shape of the pharynx, the shapes and positions of the teeth, lips, the condition of the mucous membrane of the passages generally, all affect the sound, and give it its "quality" by which we distinguish one voice from another, even if they are in the same pitch and have the same degree of loudness.

If the mucous membrane covering the vocal cords is in. 
flamed, or covered with too much mucus, hoarseness is likely to result.

As in the animal we have voice without speech, so in whispering we have speech without voice; that is, there is no true vocalization. The organs of speech so modify the aspiration as to produce speech. There is no true voice.

The voice and speech are very susceptible of culture, and nearly all voices may improve by proper cultivation. A cultivated voice and careful, distinct speech are very desirable accomplishments, and are not nearly so common as they ought to be. We learn (or think we do) to talk very early; and many individuals never give the matter any consideration, but inflict their harshness and crudity of utterance on people who cannot always defend themselves even by escape. Many a public speaker or teacher, who has fine thought, can secure but few listeners because of an unpleasant voice. He is limited to writing in his attempt to disseminate his ideas. We delight in fine singing, and many strive to cultivate this art; but not so many try to learn to talk so that it is a pleasure to hear the spoken sound.

Reading. - The Throat and the Voice, Cohen. 


\section{CHAPTER XI.}

\section{ACCIDENTS.}

\section{WHAT TO DO TILL THE DOCTOR COMES.}

How to Stop Flow of Blood from Wounds. - In case of bleeding from an artery the blood comes in jets. Pressure should be applied between the cut and the heart. To know where to apply the pressure, study of the course of the main arteries should be made. By studying Fig. 22 it will be seen that the arteries to the arms pass down the inside of the upper arm. Here they come near the surface. At the elbow the artery is near the skin in the angle of the elbow. The artery which makes the pulse at the wrist is well known. By putting a baseball under the armpit, and pressing the arm down firmly, the artery may be compressed.

In case of a deep cut in the lower part of the upper arm, a handkerchief should have a knot tied in it, and the knot placed over the artery; that is, on the inside of the arm just below the armpit. Pass the handkerchief around the arm and tie it loosely. Then run a stick through it, and twist till the knot is drawn tightly against the artery. Instead of a knot a potato, or anything else to make a firm lump, may be used. If the forearm is cut, the pressure should be applied in the angle of the elbow. (See Frontispiece.)

In studying the pulse, we found the Carotid artery in the neck. If a deep cut has been made in the upper part of the neck, it might be possible to stop the flow by compressing the artery lower down the neck. The Femoral artery comes 
near the surface in the groin. Pressure may be applied here in the same way to stop bleeding from a cut farther down the thigh. In the angle back of the knee, pressure may compress the artery supplying the leg. Nosebleed may sometimes be stopped by pressing firmly at the base of the nose.

In case of severe wounds, pressure should be applied immediately to the wound. Sometimes it is well to make a plug of cloth, and press upon the cut.

In case of bleeding from veins, holding the part up may check the flow. If necessary to apply pressure, it should be beyond the cut, instead of between it and the heart as in the case of the artery.

Blood from the lungs is bright, frothy, and salty; from the stomach is dark and sour. In case of bleeding from the lungs or stomach, let the person rest quietly on a lounge or easy-chair. Give him some bits of ice to swallow.

Bleeding from the Nose. - Do not lean forward, as this position aids the flow. Sit up, and hold up the head, and hold a cloth under the nose. Apply cold water or ice to the nose and to the back of the neck. If this does not stop it, inject cold water, with a little salt or soda in it, into the nose. Often the flow may be stopped by pressing firmly on the upper lip at the sides of the nose. If these attempts fail, a long strip of cloth may be used to plug the nostril, pushing the cloth in a little at a time, and leaving the ends so it can be pulled out; this should not be done till a long time after the flow is checked, as it may start the bleeding afresh. After an attack of this kind avoid blowing the nose, as this often starts bleeding again.

Burns. - Plunge the burned part into cold water. As soon as possible apply a solution of cooking soda (bicarbonate of soda), a tablespoonful to a teacup or tumbler of water; or lay a wet cloth on the burned part and put the soda on the 
cloth. Afterwards apply vaseline, and renew the vaseline till the wound is healed.

A mixture of equal parts of sweet oil and limewater makes a good liniment for dressing burns. Flour thickly spread over the burn is good, but probably where the flour is available, soda can also be had. Vaseline should be freely used on burns.

If the clothing takes fire, there is added to the danger of burning the body, the further risk of inhaling the flame and heated air. It is best to lie down, and roll and wrap the body in any cloths at hand, - rugs, shawls, etc. Running serves to fan the flames. Hence, if a person whose clothing is on fire is seen to be thoroughly frightened, and to have lost presence of mind and to be starting to run, the best thing to do usually is to grasp and try to throw him to the ground, putting a wrap of some kind alound the body at the same time if possible. Rolling on the ground or floor in itself would very likely put out a small flame.

Soda and dilute ammonia are good for bee stings, etc.

Fainting. - Lay the body flat on the back. Keep the crowd away. Give plenty of fresh air. Loosen the clothing about the neck and waist. Sprinkle cold water on the face (do not drench the body with a quantity of water). Apply smelling-salts (ammonia) to the nostrils ; rub the limbs toward the body. If these remedies do not soon restore consciousness, send for a physician. A faint is not usually a serious matter. $\mathrm{Bad}$ ventilation, disagreeable odors, or even the oversweet odors of such flowers as the tuberose, may cause fainting.

Broken Bones. - Keep the patient as quiet as possible till the physician arrives. There need be no anxiety if the physician is delayed, as ordinarily no harm comes from waiting. If there is inflammation, cold water may be applied. Cooling applications are desirable in case of severe bruises. 
If it is necessary to carry the patient, lay him on a board, or at least keep the injured part as quiet as possible ; a cane or umbrella may be tied alongside a leg, and supported by a pillow or a coat. Sometimes the sharp ends of the bones may cut the flesh or even blood tubes.

Sunstroke. - Lay the patient in the shade, and pour cold water over the head.

\section{TREATMENT OF THE DROWNED}

(As given by the Michigan Board of Health.)

Rule 1. Remove all obstructions to breathing. Instantly loosen or cut apart all neck and waist bands; turn the patient

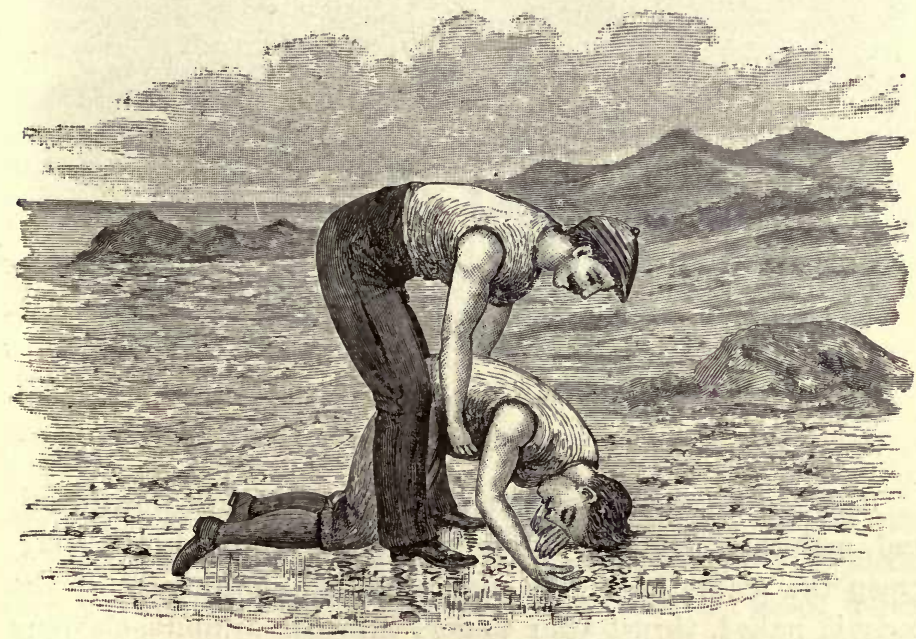

Fig. 96. Resuscitation from Drowning.

Position 1.

on his face, with the head down hill; stand astride the hips with your face toward his. head, and, locking your fingers 
together under his belly, raise the body as high as you can without lifting the forehead off the ground (Fig. 96, Position 1), and give the body a smart jerk to remove mucus from the throat and water from the windpipe; hold the body suspended long enough to count slowly one, Two, Three, Four, Five, repeating the jerk more gently two or three times.

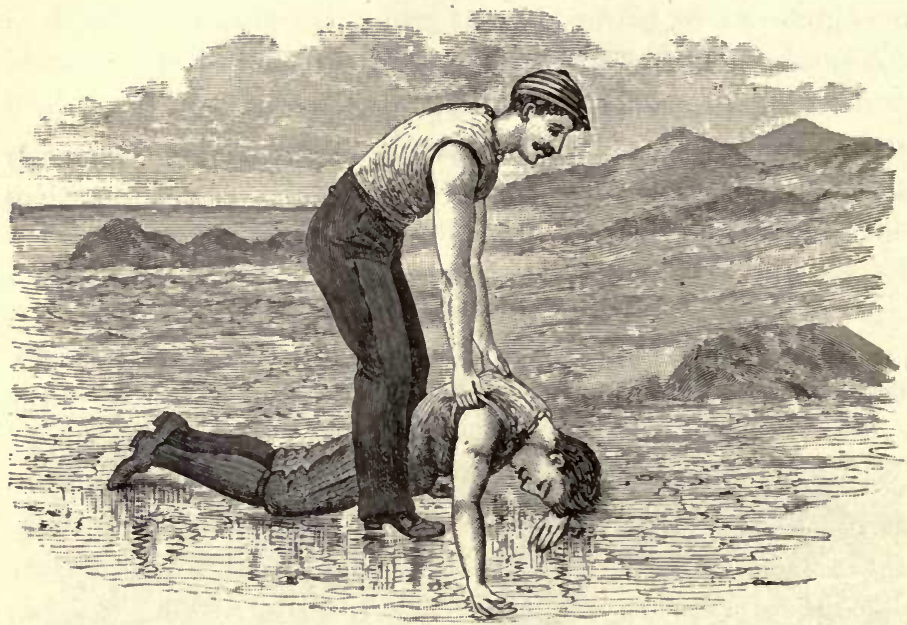

Fig. 97. Resuscitation from Drowning.

(Position 2.)

Rule 2. Place the patient on the ground face downward, and, maintaining all the while your position astride the body, grasp the points of the shoulders by the clothing, or, if the body is naked, thrust your fingers into the armpits, clasping your thumbs over the points of the shoulders, and raise the chest as high as you can (Fig. 97, Position 2) without lifting the head quite off the ground, and hold it long enough to count slowly One, Two, Three. Replace him on the ground, 
with his forehead on his flexed arm, the neck straightened out, and the mouth and nose free. Place your elbows against your knees, and your hands upon the sides of his chest (Fig. 98, Position 3) over the lower ribs, and press downward and inward with increasing force long enough to count slowly One, Two. Then suddenly let go, grasp the shoulders as before,

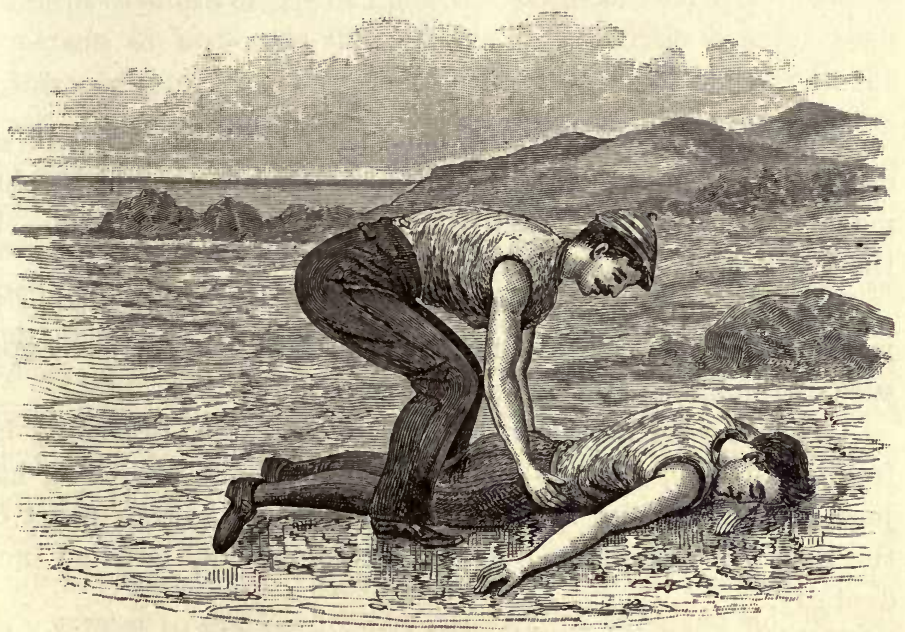

Fig. 98. Resuscitation from Drowning.

(Position 3.)

and raise the chest (Position 2), then press upon the ribs, etc. (Position 3). These alternate movements should be repeated ten or fifteen times a minute for an hour at least, unless breathing is restored sooner. Use the same regularity as in natural breathing.

Rule 3. After breathing has commenced, Restore the Animal Heat. Wrap him in warm blankets, apply bottles of hot water, hot bricks, or anything to restore heat. Warm the 
head nearly as fast as the body lest convulsions come on. Rubbing the body with warm cloths or the hand, and slapping the fleshy parts, may assist to restore warmth, and the breathing also. If the patient can surely swallow, give hot coffee, tea, milk, or a little hot sling. Give spirits sparingly, lest they produce depression. Place the patient in a warm bed, and give him plenty of fresh air; keep him quiet.

\section{BEWARE!}

Avoid Delay. A moment may turn the scale for life or death. Dry ground, shelter, warmth, stimulants, etc., at this moment are nothing - Artificial breathing is everything - is the One Remedy - all others are secondary.

Do not stop to remove wet clothing. Precious time is wasted, and the patient may be fatally chilled by the exposure of the naked body, even in summer.

Give all your attention and effort to restore breathing by forcing air into, and out of, the lungs. If the breathing has just ceased, a smart slap on the face, or a vigorous twist of the hair will sometimes start it again, and may be tried incidentally.

Before natural breathing is fully restored, do not let the patient lie on his back unless some person holds his tongue forward. The tongue by falling backward may close the windpipe, and cause fatal choking.

Prevent friends from crowding around the patient and excluding the fresh air; also from trying to give stimulants before the patient can swallow. The first causes suffocation; the second, fatal choking.

Do not give up too soon: you are working for life. Any time within two hours you may be on the very threshold of success without there being any sign of it. 
(The above, with figures, is taken from Lincoln's Iyygienic Physiology.)

Of course persons who cannot swim well ought not to go out in a boat without taking along some sort of a float that may serve as a life-preserver. Some of the rubber cushions serve well for this.

In case an ordinary rowboat is overturned, one should not attempt to climb into it or upon it. It takes very little to float a person in water, as the body is only a little heavier than water; in fact, if a person fills the lungs and lies back in the water, his face and nose will keep above water, and a person (at any rate without clothing) can float in this way for some time, if he breathes lightly. The trouble is that the person tries to lift the whole head out of the water. The dog, and such animals, when swimming, have little out of the water but the tip of the nose and a little of the top of the head. If we could learn something from them it would be a good thing. The easiest way to float is on the back. Few persons have been taught these facts; and most of those who have learned them lose their presence of mind, and waste their breath and strength in wild and fruitless splashing. If a boat be overturned, those who can swim should help those who cannot to get hold of the edge of the boat, but not permit them to climb upon it. A small plank will float a person if he will not try to lift much of his body out of the water.

Every father neglects his duty if he does not teach his children, girls as well as boys, to swim and to float. One cool, trained person may save the lives of a whole boat load.

Suffocation in Wells. - Persons are sometimes suffocated by carbon dioxid in wells and cisterns. Before going down into a well it is a safe precaution to lower a lighted candle. If this is extinguished, a warning is given. If a second person goes down after one who has become uncon- 
scious, great care must be taken that two lives are not lost. A rope should be firmly tied about the body, a hook, attached to another rope, taken to catch into the clothing of the first, and the rescuer should be lowered quickly and brought up immediately. A small rope or large cord might be carried, by pulling which the signal is given to pull up.

In resuscitating from carbon dioxid suffocation use the same method as after drowning, except the first part, which is to remove water from the windpipe, etc.

Poisons and their Antidotes. - Several of the common drugs and remedies kept about the house are more or less poisonous. The proper antidote for each should be known and kept at hand. In the first place, all such materials should be kept locked up so that they will not be taken by children, or by mistake, as in the haste of getting medicine in the night. Again, all grown persons in the family should be instructed as to the effects of each poison, and taught its antidote. As soon as any new poisonous drug is bought, it should be made a point to read up about it, and procure an antidote. Every one should know that strychnine causes spasms, that opium brings on stupor, with contracted pupils, etc.

Treatment aims at three things, (1) to get rid of the poison, (2) to neutralize what remains and prevent further action, (3) to remedy the effects already produced.

1. The most common Emetic is Mustard; a tablespoonful in a cup of warm water; give half of it, following with free drinking of warm water, then give the rest of the mustard. Do not wait for it to dissolve, but stir quickly and give at once. Provoke vomiting by tickling the throat with a feather or with the finger. If the mouth of the patient cannot readily be opened, insert the thumbs inside the cheeks and back of the teeth. If mustard is not at hand, a strong solution of table salt will serve. In a few cases, such as poi- 
soning by ammonia, lye, etc., it is considered best not to administer an emetic, but to try to neutralize the effect.

2. To neutralize a poison this general rule should be known: an alkali may be neutralized by an acid and vice versa. For example, lye with vinegar, carbolic acid with. whiting or magnesia, etc. Some acids and alkalis are always about a house.

3. After any irritant poison some mild and soothing substance should be given, - white-of-egg, milk, mucilage and water, flour and water, gruel, olive or castor-oil. These materials are partly for neutralizing the poison, and are also soothing in their effect. If a patient is drowsy, some stimulant may be given, as strong coffee (after opium). Of course a physician should be sent for immediately, as the after-treatment is of great importance.

The following tables of "Poisons, their Symptoms, Antidotes, and Treatment," are taken from the excellent Textbook of Nursing by Clara Weeks-Shaw.

Wounds from Thorns, Rusty Nails, Bites of Cats, Dogs, etc. - Promote bleeding by rubbing and pressing the wound and bathing with warm water. Or suck the wound. This tends to remove any injurious matter. Apply poultices.

If the animal is rabid (mad), suck the wound and cauterize quickly. A poker or nail heated red hot is best for cauterizing. If one cannot do this promptly, get lunar caustic with which to cauterize; strong acid or alkali, or a coal of fire, may be applied at once to the wound; the coal on a cigar may be used. Do not kill the animal if there is doubt. Keep it confined, and if it proves a false alarm much anxiety will be saved.

Snake Bites. - Apply ligatures around the part between it and the heart. Suck the wound (there is no danger in this if there are no sores or cracks in the skin of the mouth; venom 


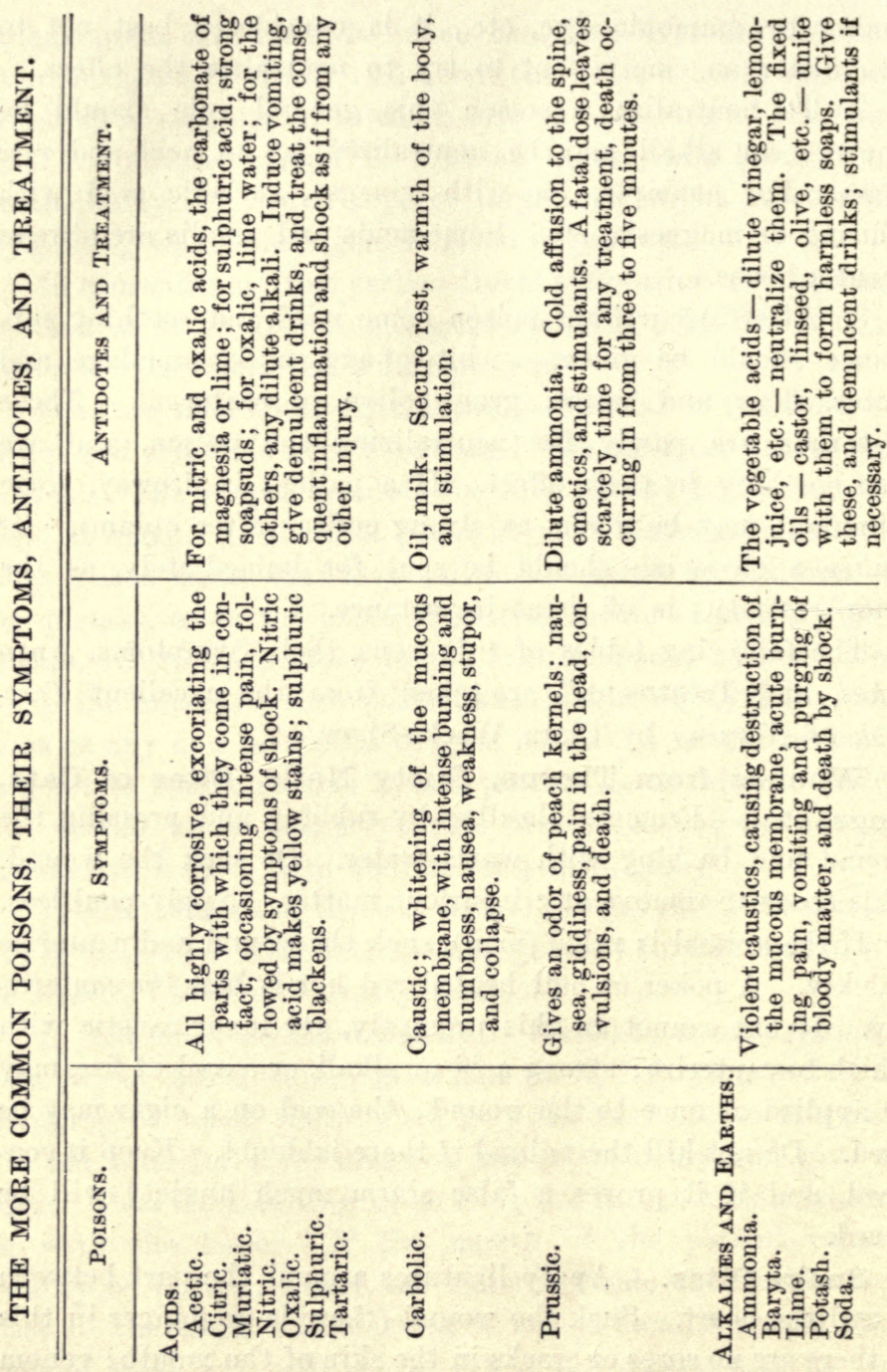




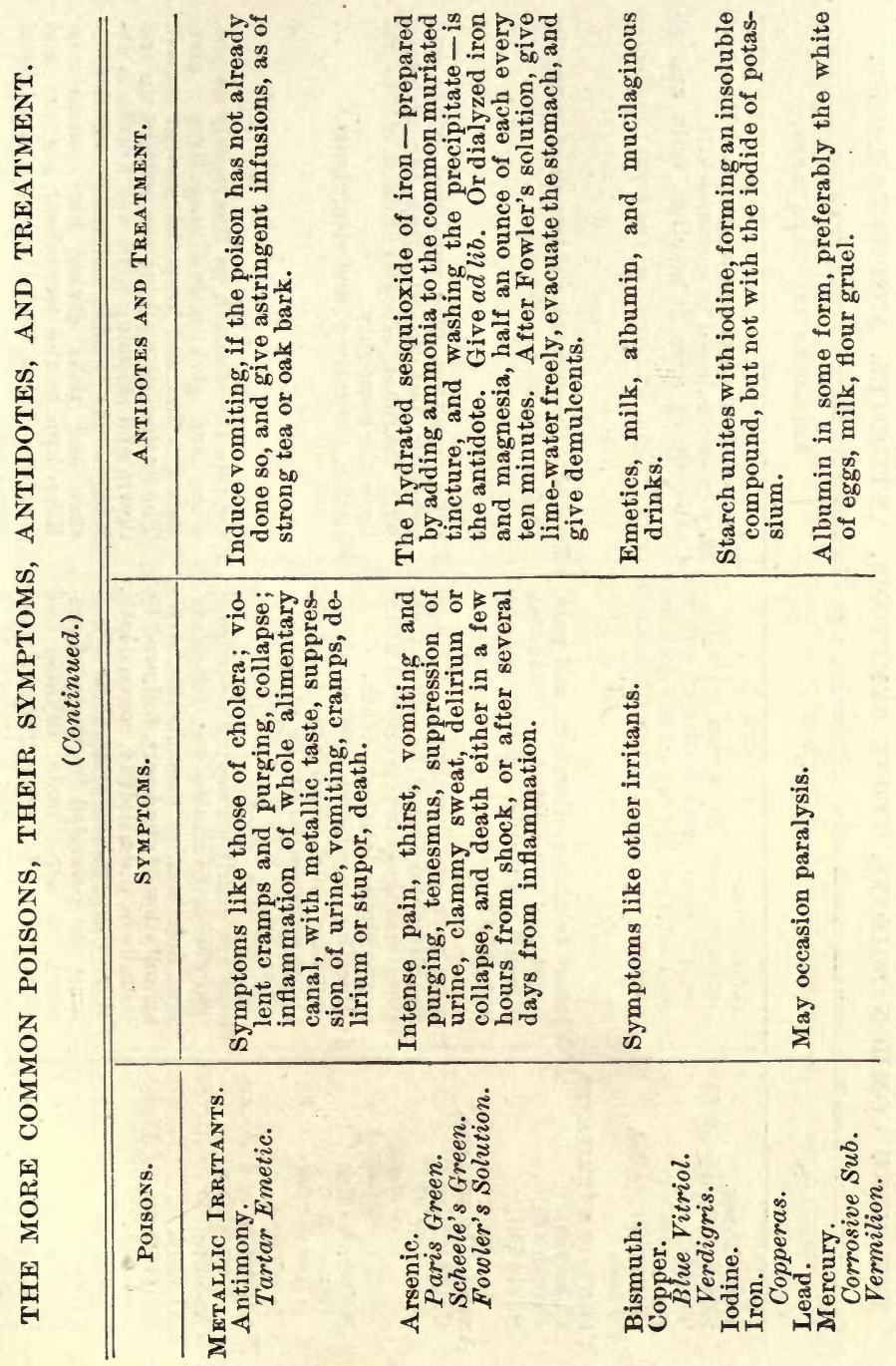




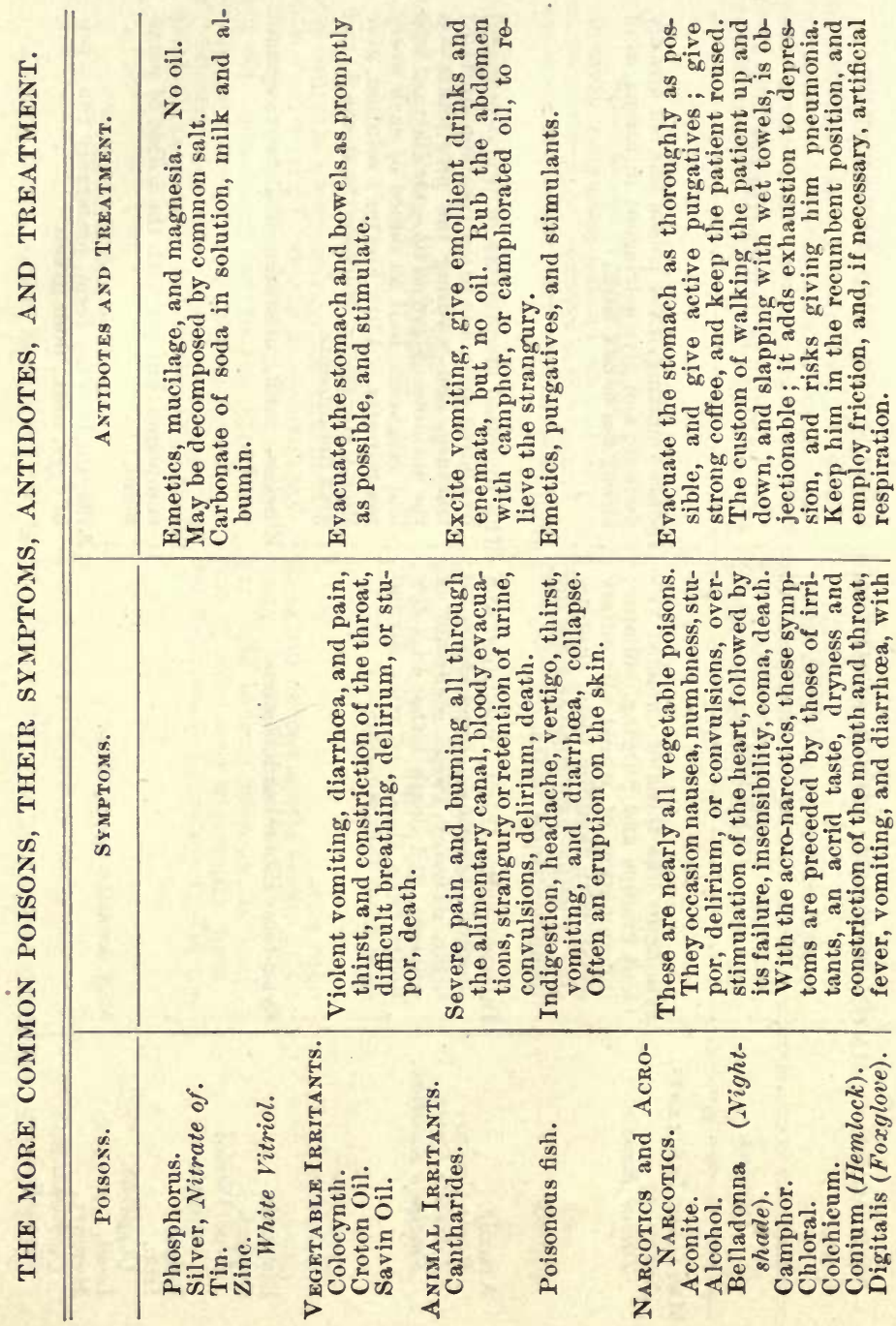




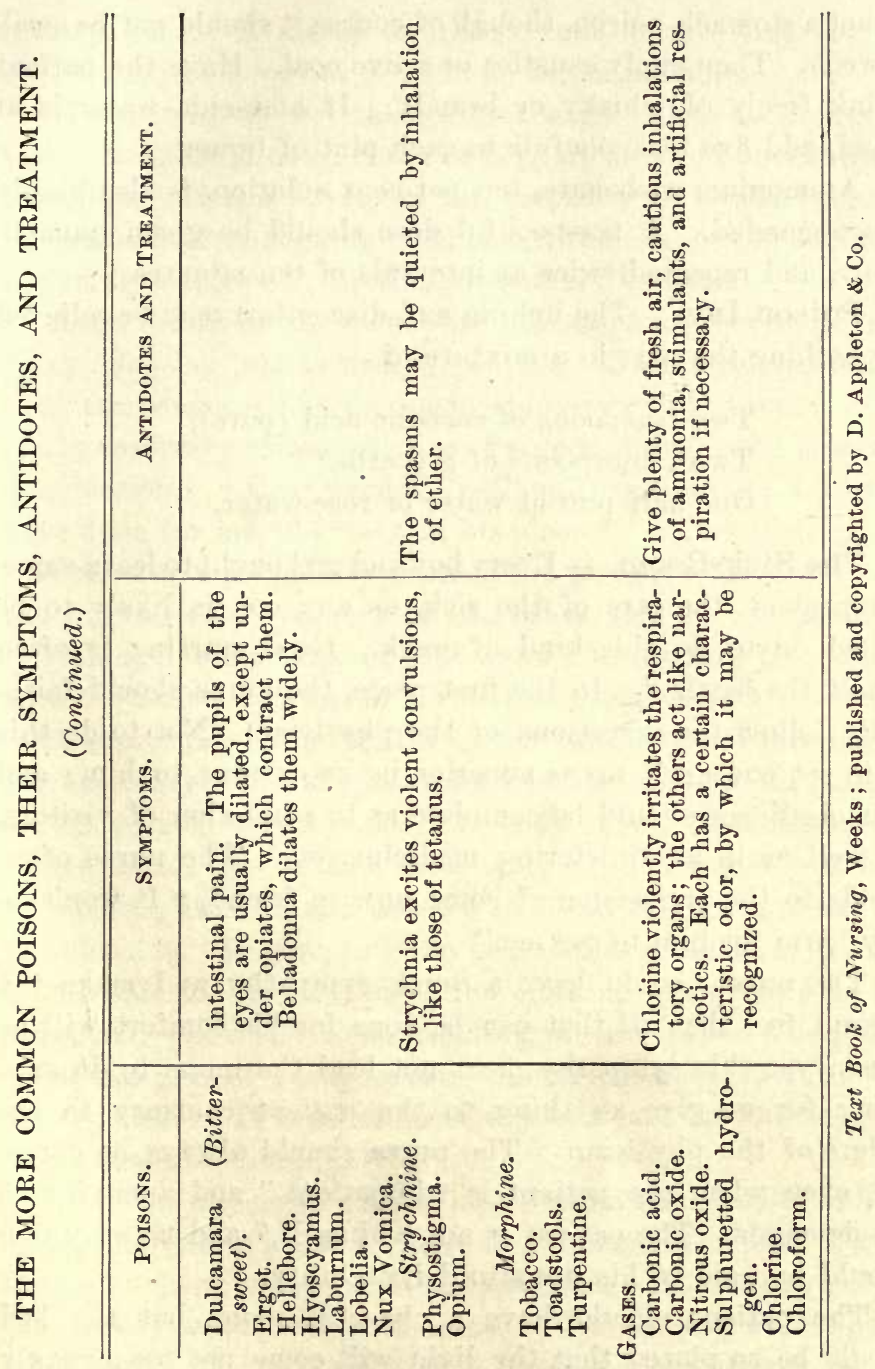


is not a stomach poison, though of course it should not be swallowed). Then apply caustics or a live coal. Have the patient drink freely of whisky or brandy. If ammonia water is at hand, add five teaspoonfuls to each pint of liquor.

Ammonium carbonate, ten per cent solution, is also highly recommended. A teaspoonful dose should be given immediately, and repeated twice at intervals of ten minutes.

Poison Ivy. - The itching and discomfort may be relieved by bathing the part in a mixture of -

Two teaspoons of carbolic acid (pure),
Two tablespoons of glycerin,
One half pint of water or rose-water.

The Sick-Room. - Every boy and girl ought to learn something about the care of the sick, as any one is likely to be called on to do this kind of work. Good nursing is often " half the battle." In the first place, the nurse should faithfully follow the directions of the physician. Not to do this is to set one's self up as superior in knowledge to him; and this obedience should be complete as to admission of visitors, as well as in administering medicine, etc. The nurse often yields to the persuasion of some unwise friend, "It won't do any harm for him to see me."

The nurse should have a quick sympathy, and make the patient feel that all that can be done for his comfort will be done; yet this sympathy must not lead the nurse to do anything for or give anything to the patient contrary to the orders of the physician. The nurse should always be cheerful, even when the patient is "impatient" and annoying in his demands. The patient is not "himself," and no attention should be paid to his unnatural irritability.

The patient should have a cheerful room, but the bed should be so placed that the light will come not too strongly 
into his face. Evidence of illness, such as medicine bottles, etc., should be kept out of sight so far as possible.

While it is not best to deceive the patient as to his condition, there should at all times be kept up an air of cheerfulness and hope. If the physician can inspire with confidence, and the nurse give unflagging good cheer, the chances of recovery are vastly improved. Nothing sustains like Hope.

Keep the air of the room pure. Remove excreta and everything offensive just as soon as possible. Do not rely on feeling as to temperature, but keep a thermometer in the room. One of the necessary characteristics of a good nurse is the power of imagination. "How would I feel, and what would I like to have done for me, if I were in his place?" This feeling will lead the nurse frequently to raise the patient's head and turn the pillow - the coolness of the other side of the pillow is refreshing; to give sips of cool water; to see that the patient does not suffer for want of a bath; in giving a bath, to do the work thoroughly, as a skillful barber carefully and thoroughly reaches every fold and crevice back of the ear, etc. In bathing a weak person, only a part of the body should be moistened at a time; after this part is thoroughly dried, another part may be washed; it is often necessary to do all this work under the bed clothing. In changing the bed clothing, move the patient to one side of the bed, push the clothing along close to his body, and place the clean bedding on the other side; then move the patient back, remove the soiled linen, and smooth out the clean. It is often necessary to warm the sheets first; they should be thoroughly dry.

Have the physician's directions written out plainly, as they may be forgotten; and if there is a change of nurses during the night there is less chance of mistake. Never let yourself get drowsy when acting as nurse. Get up and walk about, get a breath of fresh air, and if inclined to be drowsy do not 
allow yourself to settle back in an easy-chair. If watching all night, take a good lunch in the middle of the night; coffee may help to keep you awake. It is not to be expected that one who has worked hard all day out-doors will be likely to keep awake all night. There should be day and night watchers, and one would better not watch more than six hours at a time.

Do not allow the room to be swept with the ordinary broom. The room should have rugs that can be removed and shaken, and the floor wiped with a moist cloth. If the room is carpeted, it may be swept with moist salt, tea-grounds or coffee-gounds, sawdust, etc. Any dusting should be avoided; furniture may be wiped with a damp cloth.

In the effort to be quiet many make a mistake; do not whisper, as it disturbs more than talking, and also has an air of secrecy that rouses suspicion in the patient. In walking on tiptoe, often floors and stairs are made to creak when they would not in ordinary circumstances. It takes little reflection to see that in walking on tiptoe one brings more weight than usual on a smaller part of the floor, and is therefore more likely to spring a board in the floor; it is best (like the good servant Baum, in Auerbach's novel On the Heights) to walk flat-footed. Wear an easy pair of shoes; an old pair are likely to be quiet. Raise the head with the hand, or bolster the patient up, when giving drink; or if the patient is very weak, use a rubber tube, so that he will not have to lift the head. The nurse should know how to prepare any food that may be needed during the night. An oil-stove or gas-stove is very desirable for cooking, or heating poultices, as an ordinary wood or coal fire is likely to die down, making it impossible for the nurse to do this work quickly, as is often necessary to take advantage of a favorable time, as when the patient wakens, etc. Most lamps, when turned low, give off a disagreeable gas. 
It is better to have a very small lamp burning at full height than a large one turned low; sperm candles are recommended.

It is well for every one to know something about bandaging, preparation of food for the sick, etc. Space here will not allow further treatment of these subjects; and the student is referred to treatises on the care of the sick, of which there are several good ones mentioned at the end of this chapter.

It is well known that a sneeze may be prevented by firmly pressing on the upper lip. This may enable a nurse to keep from waking a very sick patient when, at a critical point, sleep is almost a question of life or death. And it is a convenient fact for any one to know. To prevent coughing, there are cough drops that will relieve the tickling in the throat. It is not right for a person who knows that he has a cold to disturb a whole congregation by coughing when, for five cents and a little forethought, he might have prevented the annoyance.

For Disinfectants see Appendix B.

In addition to the list of books on Accidents, Emergencies, etc., already given, read Hand-Book of Nursing, published under the direction of the Connecticut Training-School for Nurses, State Hospital, New Haven, Conn.; Text-Book of Nursing, Clara Weeks-Shaw; Nursing: Its Principles and Practice, Isabel Adams Hampton. 


\section{CHAPTER XII.}

\section{THE SKELETON}

- Observe that the skeleton as a whole consists of two portions, - the Axial Portion, consisting of a central axis, the spinal column, to which the head belongs; and the Appendicular Portion, the limbs and the bones belonging to them.

In the skeleton as a whole observe:-

1. The skeleton shows the form of the body.

2. It supports the softer tissues.

3. It protects softer parts, as the brain in the skull, the spinal cord in the spinal column, the heart and lungs in the thorax, etc.

4. The bones serve as levers in producing motion and locomotion.

The central part of the skeleton is the Backbone, or Spinal Column. As a whole, it is a column, widening toward the base, composed of a series of separate bones called Vertebræ.

Take a vertebra from the middle of the spinal column:-

1. Its most solid part is its body, or Centrum.

2. On the dorsal side of this is the NeuraI Arch, forming with the body the Neural Ring, through which the spinal cord passed.

3. From this arch there extend projections, or Processes. Hold the vertebra by the tip of its longest process, and place it beside the corresponding vertebra in the complete skeleton. Note that:-

(a) The body is flattened where it fitted against the vertebræ anterior and posterior to it; 


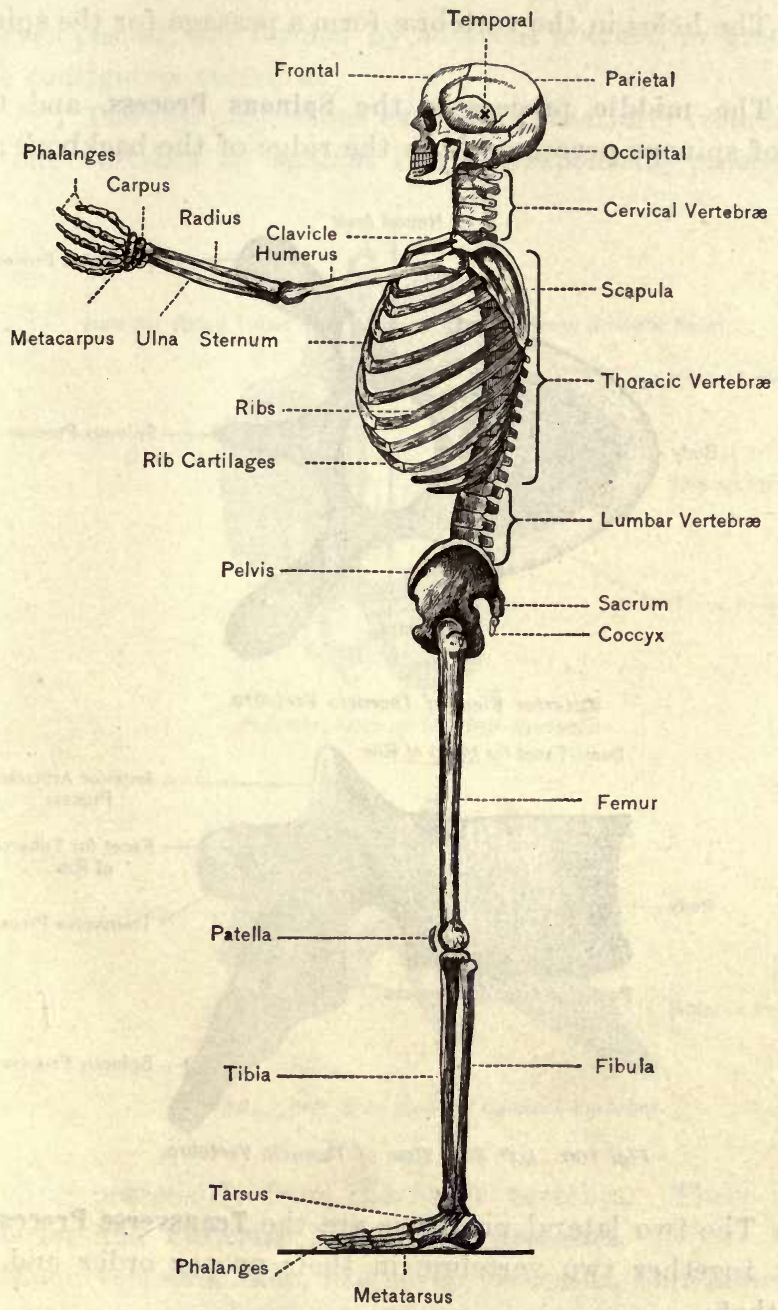

Fig. 99. Side View of the Human Skeleton. 
(b) The holes in the vertebræ form a passage for the spinal cord ;

(c) The middle process is the Spinous Process, and the series of spinous processes form the ridge of the backbone;

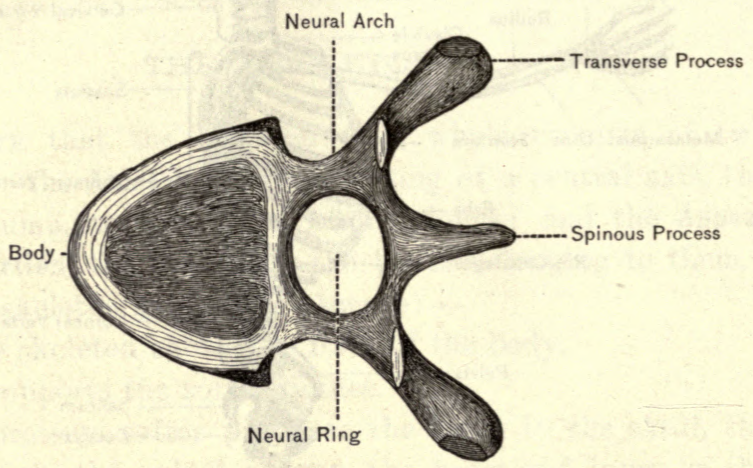

Anterior View of Thoracic Vertebra.

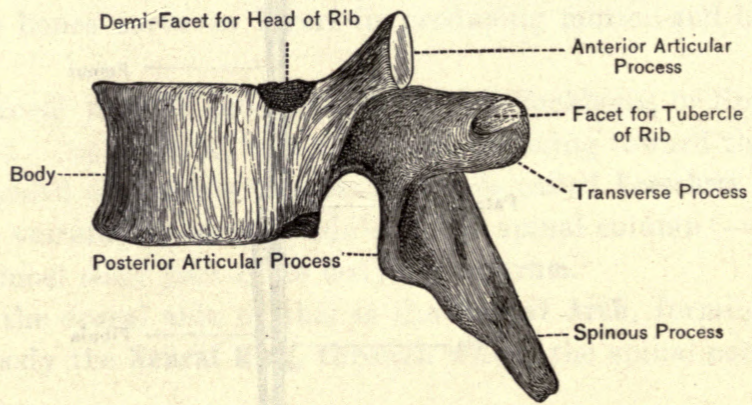

Fig. 100. Left Side View of Thoracic Vertebra.

(d) The two lateral processes are the Transverse Processes. Fit together two vertebræ in their proper order and observe that: -

(e) The openings at the sides, through which the spinal 
nerves passed, are formed by adjacent notches, or grooves, in the contiguous vertebræ.

$(f)$ The two projections extending anteriorly from the ring of one vertebra fit against two corresponding processes ex-

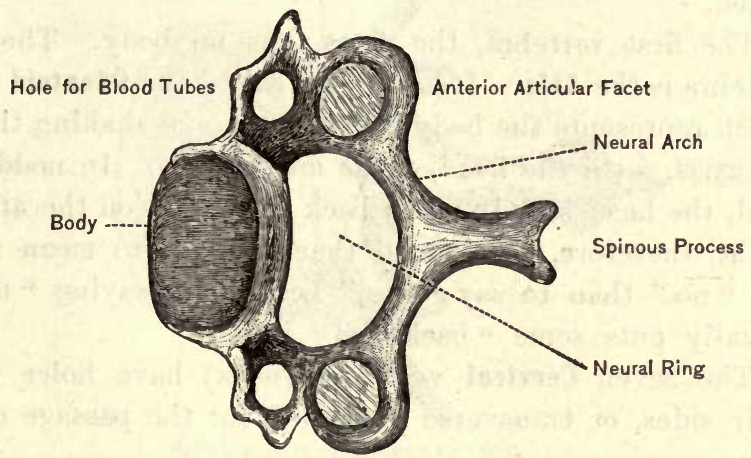

Anterior View of Cervical Vertebra

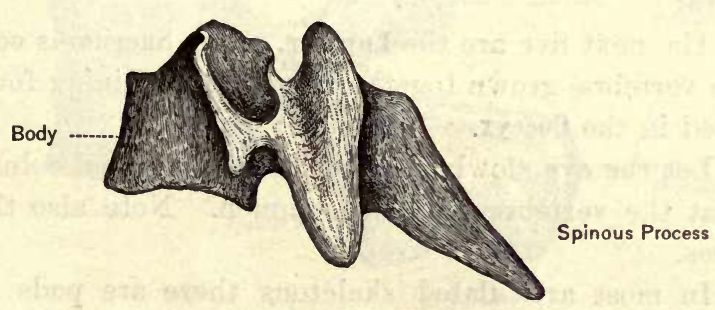

Flg. 101. Left Side View of Cervical Vertebra.

tending posteriorly from the other vertebra. These are the Anterior and Posterior Articulating Processes.

Each vertebra, then, has seven processes, four articulating (two anterior and two posterior), two transverse, and one spinous. 
The smooth places where the articulating processes join are called Facets.

Observe on each side of the body of the vertebra a facet where the head of the rib articulated. There is also a facet on the transverse process where the tubercle of the rib articulated.

The first vertebra, the Atlas, has no body. The second vertebra is the Axis. It has a peg, called the 0dontoid Process, which represents the body of the atlas. In shaking the head, the atlas, with the head, turns on the axis. In nodding the head, the head simply rocks back and forth on the atlas. It might, therefore, be claimed that it ought to mean more to say "no" than to say "yes," because in saying "no" one actually puts some "backbone" into it.

The seven Cervical vertebræ (neck) have holes through their sides, or transverse processes, for the passage of blood tubes.

The twelve rib-supporting vertebræ are the Thoracic vertebræ.

The next five are the Lumbar. The Sacrum is composed of five vertebræ grown together, and the remaining four are combined in the Coceyx.

Let the eye slowly review the whole spinal column, noting what the vertebræ have in common. Note also their differences.

In most articulated skeletons there are pads of felt between the vertebræ. These take the place of the Inter-vertebral Cartilages, which are a form of connective tissue, possessing the elasticity of cartilage and the toughness of fibrous connective tissue, such as ligament and tendon. These intervertebral eartilages serve both to keep the vertebræ apart and to hold them together. When we bend the shoulders to the right, the right edges of these cartilages are compressed, 
and the left edges are stretched, as a piece of india rubber would be if it were glued between the ends of two spools, and the whole were slightly bent.

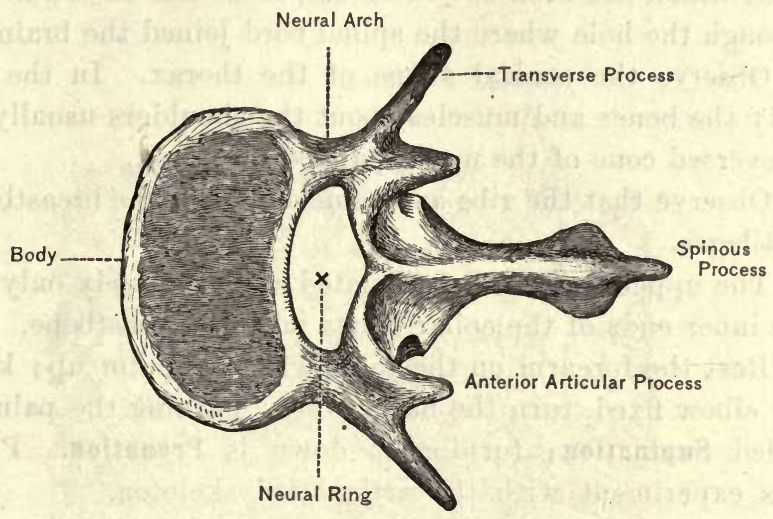

Anterior View of Lumbar Vertebra.

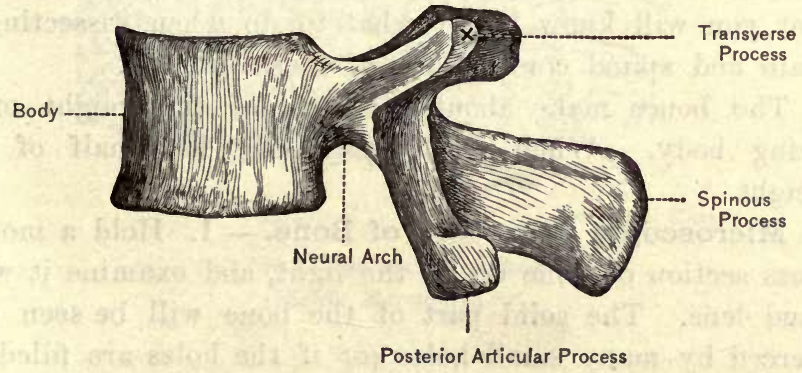

Fig. 102. Side View of Lumbar Vertebra.

View the spinal column from the side. Draw a line representing all its curves.

Take a thoracic vertebra, and, in the presence of the class, trim off the processes' with a pair of bone-forceps. The ver- 
tebra will then be seen to be, essentially, a ring, or padlock, consisting of the body and neural ring or arch.

Examine the cavity of the skull. If the class has not a skull which has been sawed across, look into the skull cavity through the hole where the spinal cord joined the brain.

Observe the conical shape of the thorax. In the entire body the bones and muscles about the shoulders usually make a reversed cone of the upper part of the trunk.

Observe that the ribs are connected with the breastbone by cartilages.

The upper limbs are articulated with the body only where the inner ends of the collar bones join the breastbone.

Rest the forearm on the table with the palm up; keeping the elbow fixed, turn the hand over. Turning the palm up is called Supination; turning it down is Pronation. Perform this experiment with the articulated skeleton.

Examine the skeleton of a cat or rabbit for the sake of comparison. Note especially the skull and spinal column, so that you will know better what to do when dissecting the brain and spinal cord in one of these animals.

The bones make about one-sixth of the weight of the living body. When dried they may lose half of their weight.

Microscopic Structure of Bone.-1. Hold a mounted cross section of bone up to the light, and examine it with a hand lens. The solid part of the bone will be seen to be pierced by many small holes (or if the holes are filled they will appear as. black spots). These are the cross sections of the Haversian Canals, through which run the blood tubes, mainly lengthwise through the bone.

2. Examine the section under the microscope, using a halfinch objective.

The bony matter will now be seen to be arranged in 
circles, Lamellæ, around the haversian canals, somewhat like the rings seen on the end of a log.

Between the rings are circles of elongated dark dots. These are Lacunæ, cavities in which lay the bone corpuscles

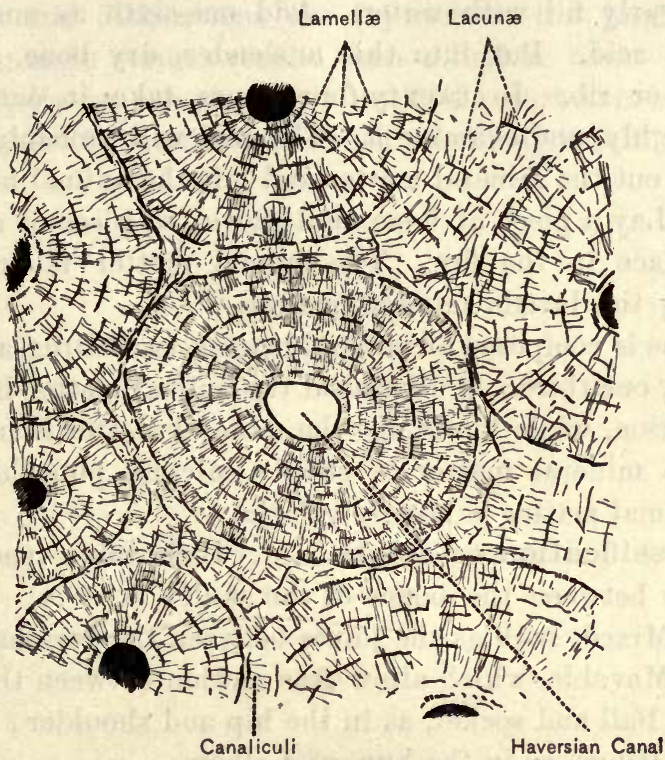

Fig. 103. Cross Section of Bone.

(Highly Magnified.)

which built up the bone. The bone was, at first, cartilage. Later, mineral matter was deposited forming true bone.

3. Now examine the section under a one-fifth-inch objective. From the lacunæ there run out, in every direction, little crevices, appearing as fine black lines. These are the Canaliculi. Through the haversian canals, lacunæ, and cana- 
liculi, the nourishing materials of the blood reach all parts of the bone.

The Chemical Composition of Bone. - 1. Take a tall, narrow glass jar, called in the chemical laboratory a "graduate," or a lamp chimney corked at one end answers very well, and nearly fill with water. Add one-sixth as much hydrochloric acid. Put into this a slender, dry bone, such as a fibula or rib. In twenty-four hours take it out, rinse it thoroughly, and examine it. The acid will probably have dissolved out the mineral matter and left the animal matter.

2. Lay a piece of bone on a shovel, or piece of sheet iron, and place in the fire. The animal matter is burned out, leaving the brittle mineral matter.

Bone is composed of mineral matter, two-thirds, and animal matter, one-third; in childhood the animal matter is in larger proportion, while in old age the mineral matter is in excess.

The mineral matter is chiefly calcium phosphate, while the animal matter is largely gelatin.

Classification of Joints. - 1. Immovable, such as the sutures between the bones of the skull;

2. Mixed, such as the joints between the vertebræ;

3. Movable, which allow free motion between the parts;

(a) Ball and socket, as in the hip and shoulder;

(b) Hinge, as in the knee and elbow;

(c) Pivot, as in the forearm, and between the atlas and axis ;

(d) Gliding, as between the short bones of the wrist and of the ankle.

Examine these joints in the articulated skeleton, and so far as possible in fresh specimens (of rabbits). Compare the ball and socket joints of the hip and shoulder. Also compare the hinge joints of the knee and elbow.

Hygiene of the Bones and Joints. - If a bone is broken, 
the person should be moved as little as possible. If necessary to move the body, care should be taken to keep the limb straight, lest the sharp ends of the bones cut blood tubes and other tissues. It is well to tie some rigid object alongside the broken limb to keep it straight.

Sprains and dislocations are injuries to the joints, and often bring more serious results than a broken bone. There should usually be complete rest until the part can be used without pain. Otherwise a stiffened joint may result. 


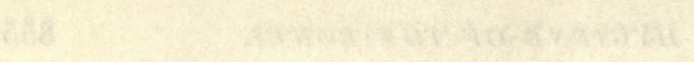




\section{A P PENDIX A.}

Tue following extended quotations are taken from Mrs. Mary Hinman Abel's Essay on Food and Cooking (which won the prize of the American Public Health Association):-

"Importance of Fats. - All the fats consumed by us, without exception, are composed of three bodies called neutral fats, mixed together in varying proportions. These three bodies are "olein," "palmitin" (margarin), and "stearin," and the chief difference between them is that they melt at different temperatures; the more olein a fat has, the more easily it melts, and the less it has, the more it is like tallow. In vegetable oils we find, in addition to these, small quantities of what are called 'fatty acids;' and in butter we have, besides the three common fats, a smaller per cent of four scarcer ones.

"Practically, therefore, all fats are alike; and when absorbed they do the same work in the body, their varying flavors and their colors having nothing to do with this.

"However, their flavor, their appearance, and the ease with which they melt in the mouth and in the digestive tract, have much to do with our estimation of them as foods. Mutton fat will do our body the same service as butter; but because of the relatively small amount of olein it contains, we have difficulty in swallowing it.

"As to the comparative digestibility of these fats, it is generally admitted that those which melt at a low temperature, like butter and vegetable oils, are most readily taken up by the system; it is thought that we could digest beeswax if it would melt in the stomach. Still, although butter stands in common estimation as the most digestible, as it is the most palatable of the fats, the stomach finds no trouble in disposing of reasonable amounts of any fats used in the household.

"The fact that all fats are so similar in composition, and that, if once digested, they will do the same service in the body, first led scientists to try to make out of the cheaper fats a substitute for butter. It was Napoleon III. who set a chemist to work to discover an artificial 
butter for use in the army. The chemist added butter color and flavors made in the laboratory to olein and margarin extracted from beef suet, and mixed with this a little real butter; and so successful was the result that the making of artificial butter has become a great industry. Now, certainly no one objects to artificial butter on the ground that it is made of animal fats, for he eats these every day on his table; he objects because he has doubts as to the cleanliness or the healthfulness of its method of manufacture.

"Therefore, since the substitution, to some extent, of animal fats for butter is, from an economic standpoint, so desirable, if we cannot bring ourselves to use oleomargarin, we must do the best we can in these kitchen laboratories of ours to make other fats than butter acceptable to the taste....

"People belonging to the well-to-do classes, unless they have given special study to the subject, seldom realize the importance of fat in our economy. Fat means to them fat meat, suet, lard, and the like, and the much eating of these is considered proof of a gross appetite; they do not consider how much fat they take in eggs, in milk, in grains like oatmeal and maize; in the seasoning of their varied dishes, and in their well-fattened meats, where, as in an average piece from a very fat mutton, they eat twice as much fat as proteid, without knowing it.

"Indeed, a well-fed man in the upper classes may have more fat in his daily diet than the freshly arrived Mechlenburg laborer who spreads a quarter-inch layer of lard on his bread. The latter cannot take his fat in unsuspected forms; he craves this principle with his plain vegetable diet, and must take it as he can get it.

"Now let us understand that where economy is to be considered, this question of fat does not take care of itself as it does for the rich man. The economical housewife should always keep in mind that she must furnish her family enough fat, and furnish it cheaply. Butter is a dear fat; count out the water in it and see what it costs you. We must economize in butter in as many ways as possible. We must eat more fat meat; first, that which is ingrained with the lean where it takes the place of water, as we have seen under 'Proteids,' costing us practically nothing; when we eat our vegetables seasoned with such a piece of meat, we find them sufficiently seasoned. We must also eat more of fat meat which we recognize as such, taking pains to cook it so that it will be palatable. The crisp, brown outside of a roast is always welcome, but the fat of boiled beef or mutton will also be relished if served 
very hot. An excellent selection of low-priced beef is the fat middle rib; the lean part is very tender and juicy when cooked in water at a low temperature for two or three hours (or in the heat-saver mentioned below); and the fat, if served very hot, any but a pampered taste will relish. Too much cannot be said in praise of pork as furnishing a goodtasting and cheap fat; it can be cooked in many ways, and used to flavor vegetables, etc.

"It is consoling to the economist to know that little of this food principle will be wasted in the body. Fat is more completely absorbed, according to the testimony of the experimenters, than any other kind of food, even meat.

"Carbohydrates, and Carbohydrate-containing Foods. As we have seen, that troublesome body, cellulose, plays here a large role. It is the skeleton, so to speak, of plants, built by them out of sugar and starch ; the chemist finds no difficulty, in his laboratory, in turning it back into dextrin and sugar, and our stomachs, too, can digest a large part of the cellulose of very young and tender plants, from 47 to 62 per cent, it has been found, of young lettuce, celery, and cabbage ; but in older plants, the cellulose proper becomes all intergrown and incrusted with substances of a woody and mineral nature, from which even the chemist separates it with the greatest difficulty, while our digestive juices are entirely unequal to the task. Therefore it is that the whole art of the cook is needed in treating this substance. She must soften it; she must break it up, and in many cases separate it as completely as possible from the sugars, starches, and proteids, which it hinders us from appropriating to our use.

"In some cases, as in oatmeal and in graham flour, we leave the cellulose, because of its mechanical action on the bowels. To be sure, this is a wasteful process, for the cellulose carries with it, when it leaves the body, considerable undigested food; but better this way than to give the muscles of our intestines so little work to do that they become unable to digest any but fine, condensed food.

"As a rule, however, we must think of cellulose not as a food at all, but as a tough foreign body which we must reckon with before we can utilize the proteid and fat particles of many important vegetable foods.

"The carbohydrates, especially the starches, are the cheapest of the food constituents, and therefore most apt to be in excess, especially in the food of the poor. According to estimates already given, an adult at average hard work gets along nicely with one and one-eighth pounds . 
of carbohydrate material (meaning, of course, the dry amount of this one principle), though fortunately, as mentioned under 'Fats,' it is found that some of this large amount can be exchanged for fat, if the body for any reason can better use the latter. Brain-workers and the richer classes the world over take less of carbohydrates, at least in their starch form, and more proteids and fats.

"It has been found that, as usually prepared, vegetable foods give up to us from one-fourth to one-half less of their nutrients than do animal foods, and especially is this true of those that are rich in proteids. To illustrate: a workman eats as a part of his dinner a dish of boiled beans; but though he rightly considers that he has been eating a nourishing dish, he has really absorbed only 60 per cent of the nitrogenous substances contained in it, the other 40 per cent passing from him unused because of its intimate connection with the cellulose.

"The starch part of vegetable food we seem to get out much better than the proteid part, even with our ordinary methods of cooking; thus, out of cooked rice we get almost 99 per cent of the starch, but only 80 per cent of what proteid it contains; flour in the form of noodles and macaroni yields up $98 \frac{1}{2}$ per cent of its starch and 80 per cent of its albumen; in the form of bread a little less of each. The potato will give us only 75 per cent of what little proteid it contains, but as high as $92 \frac{1}{2}$ per cent of its starch.

"Although the starch-containing foods are cheap, and although they yield up a good per cent of this nutritive principle, they must not be used to excess for the following reasons: Starch must first be turned into sugar by our digestive juices before it can be taken up into the blood; and if the stomach is given more at a time than it can master, certain fermentations may take place and digestion be influenced. The best authorities say that without doubt the continued and severe diarrheas of small children are due to the fermentation of starch foods for which their digestive organs are not yet ready.

"These fermentations, the irritating action on the bowels of too much cellulose, and the loss of a good deal of proteid substance connected with it, form the shady side of a regetable diet. Even the ox with his many stomachs gets out of grass and unchopped hay only 60 per cent of the proteids, and 50 per cent of the fats contained in it.

"SUgars. Most people would class sugar among the luxuries. Our first concern, however, is with its food value. It gives us the high figure of 99 per cent of the third food principle, - carbohydrates. That 
is, it must be put in the list with bread, and it can be used to a certain extent instead of bread and other starch foods. Moreover, it is especially fitted for a food in cases where nourishment is needed immediately, as it is digested or absorbed into the system almost as quickly as water, and without taxing the digestive organs, and perhaps on this account is its consumption so great in our country. We live fast, and we want our nutriment in a condensed form.

"Potatoes. We in our country need not feel as bitter against the potato as do the scientists of Europe, for we are not obliged to use it to excess; and, considering its cheapness and availability, it is for us a good vegetable, and on these accounts, though it makes a poor enough showing as to food value, we must rank it next to the bean in importance. It has only 2 per cent of proteids, no fat, and only 20.7 per cent carbohydrates; and yet since it can be prepared in so many ways, and we never tire of its mild flavor, it will doubtless continue to come upon our tables more frequently than any other vegetable. But every day, or twice a day, in large amounts, is far too often; indeed, those who use it to this extent must be ignorant of its relatively low food value. The quality of the potato is of great importance, and none but the best should be used. It should be a mealy variety and perfectly ripe.

"Fruits. Fruits are very useful to us. on account of their flavor due to various aromatic bodies, fruit acids, and sugar. The apple is especially valuable on account of its cheapness and fine keeping qualities, and is used in a variety of ways by the cook to give a relish to plain materials. Although our largest use of them is in sweet dishes, they are quite as valuable used without sugar; they may be fried in slices and eaten with fat meat, as bacon or sausage.

"The importance of dried fruits as food is not well enough understood. Fruit loses, in drying, a large portion of its water, leaving its nutritive parts in more condensed form for our use. Dried apples are very near to bread in the per cent of nutriments they offer; and the dried pear may be called the date of Germany, so general is its use. With us this fruit is too expensive, but in parts of Germany the writer has seen dried pears commonly exposed for sale by the barrel like beans. They are eaten in great quantities by the common people, who seem to digest them and dried apples without any trouble, accustomed as their stomachs are to rye bread and vegetable diet. These dried fruits are made into a variety of dishes with meats, with potatoes, and with beans, and also with noodles and macaroni. 
"Flavors or Seasonings. - So far we have had chiefly in mind the real working constituents of food, if we may so speak. But many things cannot be studied or classified in the above way; they must be looked at from another point of view.

"Thus, a pinch of pepper, a cup of coffee, a fine juicy strawberry - what of these? They may contain all five of the food principles, but who cares for the proteid action or carbohydrate effects of his cup of good coffee at breakfast, or what interest for us has the heating effects of the volatile oil to which the strawberry owes a part of its delicious taste?

"Surely the economical housekeeper who would throw out of the list of necessaries all of the things that tickle the palate, that rouse the sense of smell, that please the eye and stimulate our tired nerves, just because these things contain but little food, would make a mistake. She may know just what cuts of meat to buy, what vegetables are most healthful and economical, but if she does not understand how to ' make the mouth water,' her labor is largely lost. Especially if she has but little money should she pay great attention to this subject, for it is the only way to induce the body to take up plain food with relish.

"The list of these spices, flavors, harmless drinks, and the like, is a long one. Unfortunately we have no comprehensive word that will include everything of the sort, from a sprig of parsley to a cup of coffee; the Germans call them 'Genuss-mittel' - 'pleasure-giving things.'

"Without doubt 'hunger is the best sauce;' but it is not true, as many think, that a craving for variety is the sign of a pampered and unnatural appetite. Even animals, whom we cannot accuse of having 'notions,' have been known to starve in the experimenter's hands rather than eat a perfectly nutritious food of whose flavor they had wearied, and prisoners become so tired of a too often repeated dish that they are made sick at the sight and smell of it.

"What we call flavors may or may not be associated with a real food. Meats are rich in flavors, and each fruit has its peculiar taste; then there are the spices and aromatic herbs which are not parts of a real food; and it is most important that the cook should understand the art of adding these as seasonings to mild-tasting foods, so as to make new dishes which shall be both nutritious and appetizing. The bulk of our nourishment must be made up of the flesh of a few animals, a halfdozen grains, and as many garden vegetables; but the skillful cook can 
make of them, with the help of other flavors, an endless variety of dishes.

"An American traveling on the continent of Europe becomes acquainted with many new dishes and tastes; and although not all of them are to his liking, he must conclude that our cookery, compared, for instance, with that of the French, is very monotonous. To be sure, we may have the advantage of the European in that our markets offer us a greater variety of natural foods, especially fruits, each having a flavor of its own, and this fact makes us somewhat more independent of the art of the cook; but still we have need for every lesson of this sort, and especially is this the case with the poor, who must keep to the cheapest of food materials, which are not in themselves rich in flavor.

"Spices and other flavors, when not used to excess, stimulate our digestive organs to appropriate more easily the food to which they are added; their agreeable odor starts the digestive juices, both in the mouth and in the stomach; and their flavors, acting on the palate, have the same effect.

"The more common spices and flavors, as the housewife uses these things, are salt, pepper, mustard, cinnamon and mace, nutmeg, cloves, ginger, caraway and coriander seeds, vanilla, and many volatile oils, such as those contained in the rinds of lemons and oranges; and to this list we must add certain vegetables, as the horse-radish and various members of the onion family, the caper and nasturtium seeds, and the aromatic herbs.

"All these have their use and their abuse. Salt is hardly thought of in this list, so necessary do we consider it; and its use is well enough governed by our palate, though no doubt we over rather than under salt our food. Pepper is also in nearly every household used to excess, being added to too many dishes. The pungent mustard should be still more sparingly used; but a little of it adds relish to a salad or a meat sauce, and goes especially well with certain vegetables, as beans. Cinnamon, mace, and nutmeg, we use principally with sweet dishes, but nutmeg makes a nice variety in certain meat stews and in croquettes; foreign cooks use it far too much to suit our taste. Almost our only use of the caraway and coriander seeds is in cookies ; try the former in a potato soup for variety. Ginger seems to go well with Indian meal in pudding or porridge, and with molasses wherever used.

"To give the uses for onions and for the aromatic herbs would be too long a task. The latter can all be bought in a dried state very 
cheaply, and they retain their flavor well; one of the most useful, however, parsley, is much better fresh; by all means keep a little box of it growing in a window. Perhaps, after onion, celery is most useful for soups and stews, root, stem, leaves, and seeds being all valuable.

"In the flavoring of soups and stews, it is well to use a number of flavors, letting no one of them be prominent above the others; on the other hand, it is well to have certain favorite dishes seasoned always in the same way; as fresh pork, sage; summer savory in a bread dressing, and so forth.

"We believe that it is better to go a little high rather than too low with proteid food. As a rule, people who eat enough proteid, and especially enough animal food, are vigorous, and have what we call 'stamina;' and doctors incline to the belief that such people resist disease better because their blood and tissues are less watery than in the case of people who draw their proteids almost entirely from such vegetables as potatoes. But many workingmen in America would be surprised to learn how well health and strength can be maintained on what is, after all, not such a very large amount of meat, provided the rest of the dietary contains enough vegetable proteid and fat.

"We cannot economize in the amount of our food beyond certain limits and yet remain healthy and strong; also, we must not greatly alter the relative proportion in which experience has shown that these foods are best combined. The true field of household economy has, then, certain prescribed limits.

"Its scope lies as follows:-

"1. In furnishing a certain food principle in its cheap rather than in its dearer form; for example, the proteid of beef instead of that of chicken, fat of meat instead of butter.

" 2. Having bought foods wisely, in cooking them in such a manner as to bring out their full nutritive value; for instance, making a roast juicy and delicious instead of dry and tasteless.

" 3 . In learning how to use every scrap of food to advantage, as in soup-making.

"If we add to these the art of so flavoring and varying as to make simple materials relish, we have covered the whole field of the household economist so far as the food question is concerned.

"Few things are of more importance than that we should find ourselves, physically and mentally, equal to our day's work; but not many of us realize how largely this depends upon the food we eat. 
"Supposing there to be just enough money in a given family to buy the right kind and quantity of food. Now, if this money is not wisely expended, or if after the food has been bought it is spoiled in cooking, the results will be very serious for the members of that family; they will be undernourished, and they will suffer in clear-headedness, bodily strength, and, in case of children, in bodily development.

"Surely the right condition of the body is too important to be left to ehance ; the best scientific knowledge, the best practical head, should be at its service; and this is the case, indeed, to a large extent in Europe, where the food of the soldiers and of the inmates of public institutions is furnished more or less according to certain rules that have been deduced, partly from observation, and partly from scientific experiment.

"The application of scientific principles on these lines is not of long standing, for the investigations that have clinched them are all of comparatively recent date. At the end of the last century a beginning was made in France and in Germany in connection with the philanthropic effort to improve the food of the poor, and it was at this time Count Rumford introduced into soup kitchens in Munich the soup that has been named after him. From this time on, interest in the subject of food, both for men and for domestic animals, steadily increased, although experimenters were constantly coming to wrong conclusions because the sciences of organic chemistry and physiology, as far as they concerned the subject, were not far enough advanced.

"Every one will admit that it is of great importance for the farmer to know in what proportion he shall lay in hay and other food for winter feeding of his stock; the animals must thrive, but there must be no waste by furnishing food in the wrong quantities or proportions.

"For the housewife, the food question, in its relations to her family, can be stated in the very same way. It is important she should economize, but her path will be full of pitfalls if she does not understand in what true economy consists. Most people with a real interest in the subject have had, at some period of their lives, certain pet theories as to food. Perhaps they have been at one time convinced that most people ate too much; at another, that meat was the allstrengthener; or they may have been afflicted with the vegetarian fad ; and whatever their special views may have been, they have thought that they rested them upon facts. But surely they would never have pinned their faith to one-sided diets if they had rightly comprehended the main facts of nutrition. We believe that if these facts as at present 
interpreted, and the world's experience in applying them, can be put at the command of the housewife, she can use them to great profit.

"Soup-Making. - For one thing, we have yet to learn the great art of soup-making, and it seems also of soup-eating.

"The American housekeeper would say to me, "This is nothing new ; for years we have been hearing about soups. We don't like soups.' I only ask, 'Have you tried them for a considerable length of time, so that you have become skilled in making them and your family used to the taste?' One fact alone ought to insure for them a good trial, that at least three nations, the French, German, and Italian, make daily use of them, and have for generations. To take part of our food in this form is an absolute necessity if we are to do the best possible with a certain amount of money.

"If we do not overvalue either the gelatin or flavoring matters in our meat soups, nor throw away the meat out of which they are made we shall begin to make soups on the right basis ; that is, an understanding of the real value of the materials we are working with, and we shall use meat for our soups less often than we now do perhaps, considering its high price and our great need of it cooked in other ways. Soups should not be regarded as a luxury, neither as the last resort of poverty, but as a necessary part of a dinner, just as they are now used by all classes in Europe; but they need not be made of good cuts of meat, nor indeed, of meats at all.

"Lean meat of any sort, beef best; fresh, better than that long kept; bones of next value, especially the spongy rib bones and vertebræ. Saw and chop the bones into little pieces; cut the meat small. Soft water is better than hard.

"Keep a kettle, if possible, for this purpose alone, and add to it all bits of meat and bones as they accumulate. Put the meat into cold water, let it stand some hours if possible, heat very gradually, and keep simmering. Two hours or less brings ont all the flavors of the meat, but a much longer time is necessary to get all the nutriment from the bones.

"Do not remove the scum ; it contains the albumen of the soup, and nothing objectionable if the meat was well cleaned.

"An hour before the soup is served add flavors; onions and carrots are the best, celery, summer savory, and parsley next. Use others, as cloves, nutmegs, bay leaf, etc., only occasionally. Add salt and pepper just before serving. 
"When done, strain and skim off all fat (better if left to stand till next day, the fat removed, and the soup simply rewarmed), and make such additions as you wish.

"The Heat Saver. - It is a part of common information that the inhabitants of northern countries make extensive use of non-conducting substances, like wool, for preventing the escape of heat from a vessel in which cooking is going on. It is strange that we do not make more use of such appliances, for they have often been described and illustrated ; it is probably because they are not found ready made, and with a complete list of directions for use. The writer made and used a cooker of this sort, and after considerable modification and experiment it became a very useful thing in the kitchen. If you wish to cook meat at the proper temperature, this contrivance makes it possible to do so, and is also very saving of fuel.

"Take a packing-box measuring, say, two feet each way, and cover the bottom with a layer of packed wool four to six inches thick; set into the middle of this another box or cylinder of sheet iron, and fill the space between the two with a layer of wool four to six inches thick, and closely packed. Into the inner compartment put your kettle of meat or vegetables already brought to the boiling-point and having a tightly fitting cover, and over this press a thick pillow or woolen blanket. Then fasten down, tight over all, the lid of your box. As the heat in the water must finish the cooking already begun, this amount must be rightly proportioned to the amount of food to be cooked ; for example, two quarts of water to one and one-half pounds of beef rib were used. The water was brought to the boiling-point, the meat placed in it, and allowed to boil for five minutes, the pot was then tightly covered, placed in the box and allowed to remain three hours. At the end of that time the meat was tender.

"Introduction to Bills of Fare. - (To the Mother of the Family.) In the general introduction the writer has stated a few principles that should guide us in choosing our food. We have learned that to keep us in good health and working order we ought to have a certain amount of what is best furnished by meat, eggs, milk, and other animal product, and that we must also have fat as well as what is given us in grains and vegetables.

"But now our work has only just begun ; for we are to furnish these food principles in the shape of cooked dishes to be put on the family table three times a day; and the dishes must not only be nourishing, but 
they must taste good, and there must be plenty of variety from day to day; and last, - and this is the hardest point of all, - we are to do this for the sum of thirteen cents per person daily.

"I am going to consider myself as talking to the mother of a family who has six mouths to feed, and no more money than this to do it with. Perhaps this woman has never kept accurate account, and does not know whether she spends more or less than this sum. She very likely has her 'flush' days and her 'poor' days, according to the varying amount of the family earnings; and it may be a comfort to her to know that if she could average these days and plan a little better she can feed her family nicely on this sum.

"A few facts as to what the writer knows to have been done in this line will not be amiss. I knew a family of six belonging to one of the professional classes, half grown people and half children, that lived for a year on an average of eleven cents per person daily, and no one would have said that they did not live well enough ; and they had meat about four days out of the seven; there was always cake on the supper table, and they used plenty of fruit.

"Here is an average bill of fare : Breakfast-milk toast, fried potatoes, coffee ; dinner - soup made of shank of beef, fried liver, rice with potatoes; supper - bread and butter, fried mush, stewed pears, and cake. Next day there was pressed beef made from the soup meat chopped and flavored, and next there was cheap fish nicely fried. The head of this household was a skillful economist, absolutely no mistakes were made in cooking, and not a scrap was wasted. She had a long list of simple dishes at her command, and she specially studied variety. 'I abandon even a favorite dish for weeks,' she said, 'if any one tires of it.' I give this as a sample of what I know to have been done by a highly respectable family in a city of small size in one of our Eastern States.

"It must be mentioned that the price on which this family lived in comfort could not have been so low as it was but for one great help, they had a garden that furnished green vegetables and a little fruit. But then most every family has some special advantage that would lower the rate somewhat; one buys butter or fruit advantageously of friends in the country, another can buy at wholesale when staples are cheaper, still another may be able to keep a few fowls, and so on. Numerous instances could be brought to prove that the food for a family can be purchased in a raw condition for the sum per head for which 
we have undertaken to buy it, and that skill in cooking and flavoring, and by giving right variety, a healthful and acceptable diet can be furnished, though it cannot, of course, cuntain luxuries.

"Another thing, when I speak of a woman who is to buy the food of a family for thirteen cents aprece daily, I have in mind the wife of a man who earns this sum himself, the wife having her time to attend to her housework and childreil. If a woman helps to earn, as in a factory, doing most of her housework after she comes home at night, she must certainly have more money than in the first case in order to accomplish the same results, for she must buy bread already baked, and can only cook those dishes that take the least time.

"I shall take for granted that you have the kitchen utensils described on page twenty ; if not, buy them, because you cannot afford to be without them. Food is very expensive compared with pots and pans; you must not spoil food for lack of the right things to cook in.

"I only ask you in advance to try the recipes I shall give you, and to try and lay aside your prejudices against dishes to which you are not accustomed, as soups and cheese dishes, for instance. You cannot afford to reject anything that will vary your diet, for many good-tasting things you cannot buy. I know it is hard for a busy woman to give her cooking a bit more time than will 'just do,' but if you make it a rule to determine the night beforehand just what you will cook the following day, no matter how simple the food may be you will gain this result; with the materials at your disposal you will put before your family nuch better food, and they will call you a good cook, and think that no family need live better than they; and this impression will be made from your having the right variety. Let us understand, to begin with, that it is your business in life just now to conquer the food question as it affects your family. Just as the business man must watch the market and take advantage of a half cent a pound on an article that he may successfully compete with his neighbor, so you must be on the alert to use every possible advantage. It is a struggle in which energy and calculation will tell for a great deal, and you will have solid enjoyment in every point you gain.

"In buying meat, your saving cannot be so much in quantity as quality. Try to learn the different parts of the animal, and to distinguish between meat from a fat ox and a lean one; for, as we have explained, the former has less water in it, and why should you pay good money for that which nature gives you free? In winter try to 
buy meat ahead so that you can make it tender by keeping it; and yon will notice, too, that the larger the piece you buy, the smaller the per cent of bone you get with it.

"The per cent of bone in the whole animal, as in the case of an ox, is not more than ten to eleven per cent; but the buyer of a small piece often gets twice that proportion. As we have said again and again in these pages, the low-priced or tougher parts have as much nutriment for you as the rib roast which is beyond your purse. Choose often the fat middle rib, and cook it long and slowly ; buy the neck and scrag of mutton, and make a stew with vegetables; buy half a calf's head, and see what a fine soup you can make of it. Have beef's liver now and then and tripe, rather than put your money into sausage of doubtful quality. By all means buy fish when it is cheap, catfish for instance, which are excellent fried. Keep suet always on hand, and use it instead of butter, as has been directed.

"No one need tell you how valuable salt pork and bacon are for you; the only danger is that yuu will use too much of them.

"In buying eggs, you must be governed by the price; in winter use as few as possible, and even in the spring, when they are cheaper, remember that they are not as cheap as the lowest priced cuts of meat from fat animals. But when they cost only fifteen cents a dozen you can well disregard any small comparison of nutritive values in consideration of their high worth in furnishing variety ; you can afford to use them now and then in place of meat, and in making various egg dishes.

"Of the value of cheese to take the place of meat, you can read in another part of this essay. Buy it once a week at least, the skim variety if you cannot afford the other, and grate and cook it according to the recipes given.

"Try to find a reliable milkman, and buy skim milk at half the price of full, and use it for all cooking purposes, keeping full milk, and if possible a little of the cream, for coffee.

"Now let us take the vegetable part of your diet. You must keep on hand every kind of flour and grain that is not too expensive; be thankful that wheat flour is so good and cheap, it will be your best friend. If you are not already skillful in the use of doughs, you will waste your materials and make mistakes at first; but there is nothing for you but to become mistress of this department of cookery. Use bread freely in all bread dishes; learn how to make every one. You 
will use buckwheat for cakes, rice for puddings, barley in soups, oatmeal and cornmeal for mushes; you must learn to use them all in as many ways as possible. The grains are cheaper foods for us than vegetables, although dried peas, beans, and lentils follow hard upon them. Even the potato, which may be called our favorite vegetable, is more expensive than wheat flour, if we are talking only of food values.

"Except in the height of their season have nothing to do with green vegetables, at least not under the impression that they are cheap; if you buy them, know that you are paying for flavors and variety rather than for food. But even in the early spring buy plenty of such vegetables as onions, carrots, parsley, and other green herbs for your soups and stews. When you go for a walk in the country, be sure to bring home some mint and sorrel in your pocket; the former will make a nice meat sauce, the latter a delightful flavor in soup. It will be perfectly easy for you to grow in the window-box that delicious herb parsley, and have it always fresh.

"For a low purse there is no help so great as a knowledge of flavorings. When we remember that we can live on bread, beans, and peas, and a little cheap meat and fat the year round, if we can make it 'go down,' we shall realize the importance of such additions as rouse the appetite; there is room here for all your skill and all your invention. Always make a cheap but nutritious dish inviting in appearance ; especially does this influence the appetites of children, who are delighted with a very plain cake if only a few raisins or some sugar appear on the top.

"The bills of fare (given below) where seventy-eight cents covers the cost of food for a family of six per day, and where the amount of food is carefully weighed and estimated, is meant to suggest to you how in a few cases your food problem can be solved. You can, no doubt, spend the money in ways that will better suit the tastes of your family; but I beg you to examine anew your favorite dishes to see if they are as nutritious as they should be for their price. Remember that the proteid column is the one you must look to most carefully, because it should not fall below the figures I give. If, for instance, you should economize on meat in order to buy cake and pastry, this column would suffer at the expense of the other two, and the family would be undernourished." ABEL.

The Aladdin Oven. - Of all the appliances for cooking that are known to the writer, the most valuable is the "Aladdin Oven," in- 
vented by Edward Atkinson. It consists of a sheet-Iron oven, with snugly fitting door. This is placed within an outer casting made of non-heat-conducting material. There is a space between the inner oven and the outer box, and between the two heat is held and applied to the work of cooking. Since little heat is lost, not a great amount is required, and a common kerosene lamp supplies all the heat that is needed. The lamp is placed beneath the oven under a hole in the outer box. This hole is four inches across, but is usually reduced to two and a half inches by a lid fitting over it. It will probably be a matter of surprise to those who hear it for the first time, that bread can be baked in such an apparatus, but the writer's experience is that of others who have tried ; namely, not only that it is repeatedly done, but that the bread thus cooked is superior to bread baked in an ordinary oven. The cooking in the "Aladdin Oven" is slow cooking. Many of the scientific cooks repeatedly tell us that we must not employ too high a heat in cooking. And this slow cooking at a lower temperature gives better result in nearly every kind of cooking. Mattieu Williams, in his Chemistry of Cookery, tells us that "to boil meat is to spoil it." And the description of the "Heat Saver," given on page 367 illustrates the gain in saving of fuel, and in the better condition of the food after it is cooked. Mrs. Ellen H. Richards, one of the best authorities on this subject, in speaking of the requirements of an ideal cooking apparatus, says :-

" 1 . The degree of heat should be under perfect control ; increased, diminished, or withdrawn at will, and without' loss of time. Solid fuel demands constant and equable running, and gives the best results when used in large masses. The small fire-box of a cook-stove, and the urging of the fire for a short time three times a day, are fatal objections to the use of anthracite.

"2. A tightly closed vessel heated by steam, or hot water, or hot air, offers many advantages over the top of a red-hot stove or the inside of a nearly red-hot cast-iron oven for cooking, except for the broiling and the roasting of meat, and for some other methods of cookery which require the quick application of heat.

" 3 . For all purposes of slow cooking, the oven should have a nonconducting covering which retains the heat where it is wanted, and also allows of tight closing, and of security from the constant watching required by the fitful heat of a stove. This use of a close oven with a non-evaporative atmosphere seems to be the secret of the retention 
of the delicate and volatile flavors which usually flavor the house and street, and not the food as it is brought to the table."

After thus outlining the ideal cooker, she goes on to say, "For simplicity, effective use of heat, economy of fuel, and development of flavor in the food cooked, combined with increase of its digestibility, the 'Aladdin Oven' is an apparatus far exceeding in merit any other now on the market."

Among the many advantages of the "Aladdin Oven" the following may be named :-

1. The greater digestibility of the food cooked in it. In the high heat of the ordinary oven many foods are so altered by the great heat as to become very indigestible. This is notably so in the case of fat. The high heat in some way breaks up the fat so as to make it exceedingly indigestible and injurious to most stomachs. The more moderate heat of the "Aladdin Oven" cooks fat pork and bacon so that even delicate stomachs find it palatable and digestible. In the use of this cooker in his own family the writer knows that many dishes, cooked by this slow process, are readily taken and assimilated, which, when cooked in the common stove, were difficult of digestion.

2. Another feature is its economy. (1). In economy of food, little is spoiled, as it is difficult to overcook or burn anything. (2) Economy of fuel. A quart or so of kerosene will do the cooking for a family for a day. (3) Economy of time and labor. In much of the cooking on the ordinary stove the food must be constantly watched, often stirred, while the cook is subjected to a very uncomfortable heat. Most dishes can be put into the "Aladdin Oven," and left entirely alone until they are completely cooked. Of course it takes some experience to know just what degree of heat to apply, and how long it takes for a given food or a given weight to be cooked. For instance, much of the cooking can be done at night. The materials for breakfast being put into the oven in the evening, will be all ready for breakfast, and hot for either a late or an early breakfast. The comfort, especially in the summer, and convenience to the cook, can hardly be overestimated.

3. Using a liquid fuel makes it as easy to regulate as a conmon lamp ; there are no ashes, no dust, no more odor than from a lamp, and that is hardly noticeable if the lamp is kept clean. Any contrivance that will enable one to set the breakfast to cooking before he goes to bed, with no more trouble, so far as the fire is concerned, than to light a common lamp and place it under the oven; and in the morning to 
have little more to do than to transfer the dishes from the oven to the table (for many foods can be cooked in the same dishes in which they are served); certainly every family where time and labor are worth economizing should hail this apparatus with delight. It is the wonderful lamp of "Aladdin" that brings Health, Comfort, and Economy.

The inventor seeks no gain for this ingenious cooker. He has donated his patent to the world. He has also supplemented the oven by a very valuable treatise on The Science of Nutrition and the Art of Cooking. (Published by Damrell \& Upham, Boston, Mass.)

Readiva. - Practical Sanitary and Economic Cooking, Abel; The Science of Nutrition and the Art of Cooking, Atkinson; Chemistry of Cookery, Williams ; Chemistry of Foods and Nutrition, Atwater (Century Magazine, 1887-88); Eating for Strength, Holbrook; Foods, Smith ; Disorders of Digestion, Brunton; Indigestion and Biliousness, Fothergill ; Philosophy of Eating, Bellows ; Handbook of Invalid Cooking, Boland. 
SAMPLE OF BILL OF FARE AND MATERIALS FOR FAMILY OF SIX, . AVERAGE PRICE 78 CENTS PER DAY, OR 13 CENTS PER PERSON. -(ABEL.)

\section{Monday, May.}

BREAKFAST.

OATMEAL MUsh, With • Milk aNd Sugar.

BREAD.

CoffeE.
DINNER. PeA Soup (p. 117).* Mutton Stew (p. 52). Boiled Potatoes. BREAD.

SUPPER.

Bread Pancakes (p. 93).

Fried Bacon.

TEA.

\begin{tabular}{|c|c|c|c|c|c|}
\hline & & $\begin{array}{l}\text { Pro- } \\
\text { TEIDS } \\
\text { Oz. }\end{array}$ & $\begin{array}{c}\text { FATS } \\
\text { oz. }\end{array}$ & $\begin{array}{c}\text { CARBO- } \\
\text { HYDRATES } \\
\text { OZ. }\end{array}$ & $\begin{array}{c}\text { Cost } \\
\text { IN } \\
\text { CENTS }\end{array}$ \\
\hline 2 Eggs . • . & - & .34 & .32 & . & 3 \\
\hline $3 / 4$ lb. Oatmeal . & - & 1.74 & .72 & 7.80 & $3 \frac{3}{4}$ \\
\hline 1/8 lb. Coffee . & - & - & - $\cdot$ & - & 32 \\
\hline 1/2 lb. Sugar . . & - & - & • & 7.92 & $3 \frac{1}{2}$ \\
\hline $11 / 2$ qts. Skim Milk & $\mathbf{k}$ & 1.59 & .36 & 1.48 & 6 \\
\hline $3 / 4$ lb. Bacon. & - & .36 & 9.60 & - & 9 \\
\hline 4 lbs. Potatoes. & . & 1.28 & - & 13.24 & 5 \\
\hline 4 lbs. Bread . & - & 3.84 & .32 & 33.20 & $9 \frac{1}{5}$ \\
\hline 1 qt. Whole Milk & . & 1.16 & 1.24 & 1.66 & 7 \\
\hline 3 lbs. Shoulder of 1 & Mutton & 8.16 & 2.88 & . & 21 \\
\hline 1 lb. Peas, Dried & - & 3.68 & .32 & 8.32 & 5 \\
\hline $1 / 2$ lb. Flour . & - & .96 & .12 & 5.72 & $1 \frac{1}{2}$ \\
\hline Total . . & . & 23.11 & & 80.34 & 77.3 \\
\hline Required. & . . & 19.19 & 12.42 & 78.03 & 78 \\
\hline
\end{tabular}

* Page numbers refer to Mrs. Abel's Practical Sanitary and Economic Cooking. 
SAMPLE OF BILL UF FARE AND MATERIALS FOR FAMILY OF SIX, AVERAGE PRICE \$1.38 PER DAY, OR 23 CENTS PER PERSON.-(ABEL.)

\section{Monday, JANUARY.}

BREAKFAST.

BuCKWHEAT CAKEs.

SAUSAGE.

COFFEE.

APple SAUCE.
DINNER.

PeA Soup (p. 118).

ROAST BEeF.

BaKed Potatoes.

Canned Tomatoes.

Barley Gruel (p. 121).

SUPPER.

Potato Soup with Egg and Bread Balls (p. 128). Brown Bread and Butter.

Canned Fruit. Tea.

$$
\begin{aligned}
& \text { Pro- Fats, Carbo- Cost } \\
& \text { TEIDS. FATS. HYDRATES. IN } \\
& \text { oz. oz. Oz. CENTs. }
\end{aligned}
$$

$\begin{array}{lllll}2 \text { lbs. Buckwheat Flour . } & 3.04 & 64 & 23.20 & 10\end{array}$

1 lb. Sausage $\quad . \quad$. . $\quad \begin{array}{llll}2.32 & 6.00 & \ldots & 12\end{array}$

2 lbs. Beef . . . . . $6.72 \quad 1.76 \quad \ldots .62$

3 lbs. Potatoes . . . . $\quad .96 \quad$. $\quad 9.94 \quad 3 \frac{3}{4}$

2 lbs. Tomatoes (canned at home) . . . .

3 lbs. Bread ... . . 2.88

2 Eggs . . . . . . . . 34

$1 / 2$ lb. Barley . . . . .88

1 qt. Whole Milk . . 1.16

$1 / 2 \mathrm{lb}$. Sugar ..... .

$1 \mathrm{lb}$. Dried Peas . . . 3.68

$1 / 2$ lb. Butter . . . . . .

Canned Fruit .. . . . .

$1 / 8 \mathrm{lb}$. Coffee .......

Tea

Tea

Apples

Total

Required . . . : . 19.19
. 3.50

$.24 \quad 24.90$

.32

.12

1.23

.

.32

6.66

c.

7.90

5.72

1.65

7.92

8.32

5

.. $12 \frac{1}{2}$

.. 10

.. $\quad \ldots \quad 3 \frac{3}{5}$

$\begin{array}{lll}\cdots & \ldots & 35 \\ \cdots & \ldots & 2\end{array}$

.. .55

$\overline{17.29}$

85.15

$127 \frac{1}{5}$

12.42 


\section{APPENDIX B.}

Antiseptics and Disinfectants. - The following is chiefly from Sternberg's Manual of Bacteriology, and embodies part of the report of "The Committee on Disinfectants of the American Public Health Association."

An Antiseptic is a substance having the power to prevent or destroy putrefaction, or, what is the same thing, the bacteria upon which putrefaction depends.

Disinfection is the destroying of disease germs by means of heat, chemic substances, fumigation, or by fresh air or sunlight.

"The injurious consequences which are likely to result from such misapprehension and misuse of the word 'disinfectant' will be appreciated when it is known that recent researches have demonstrated that many of the agents which have been found useful as deodorizers or as antiseptics are entirely without value for the destruction of disease germs.

"This is true, for example, as regards the sulphate of iron, or copperas, a salt which has been extensively used with the idea that it is a valuable disinfectant. As a matter of fact, sulphate of iron in saturated solution does not destroy the vitality of disease germs, or the infecting power of material containing them. This salt is, nevertheless, a very valuable antiseptic, and its low price makes it one of the most valuable agents for the arrest of putrefactive decomposition."

Extracts from the Above-Mentioned Report.

"The most useful agents for the destruction of spore-containing infectious material are:-

1. Fire ; complete destruction by burning.

2. Steam under pressure, 105 degrees C. (221 degrees F.), for ten minutes.

3. Boiling in water for half an hour.

4. Chlorid of lime ; a four per cent solution. 
5. Mercuric chlorid; a solution of 1.500 .

For the destruction of material which owes its infecting power to the presence of micro-organisms not containing spores, the committee recommends :-

1. Fire ; complete destruction by burning.

2. Boiling in water for ten minutes.

3. Dry heat ; 110 degrees C. ( 230 degrees F.) for two hours.

4. Chlorid of lime; a two per cent solution.

5. Solution of chlorinated soda ; a ten per cent solution.

6. Mercuric chlorid; a solution of $1: 2,000$.

7. Carbolic acid; a five per cent solution.

8. Sulphate of copper; a five per cent solution.

9. Chlorid of zinc; a ten per cent solution.

10. Sulphur dioxid; exposure for at least twelve hours to an atmosphere containing at least four volumes per cent of this gas in the presence of moisture.

The committee would make the following recommendations with reference to the practical application of these agents for disinfecting purposes:-

For Excreta. - (a) In the sick room:-

1. Chlorid of lime, four per cent.

In the absence of spores:-

2. Carbolic acid in solution, five per cent.

3. Sulphate of copper in solution, five per cent.

(b) In privy vaults:-

1. Mercuric chlorid in solution, $1: 500$.

2. Carbolic acid in solution, five per cent.

(c) For the disinfection and deodorization of the surface of masses of organic material in privy vaults, etc.: -

Chlorid of lime in powder, or freshly burned quicklime.

For Clothing, Bedding, etc. - (a) Soiled underclothing, bed linen, etc.

1. Destruction by fire, if of little value.

2. Boiling for at least half an hour.

3. Immersion in a solution of mercuric chlorid of the strength of 1: 2,000 for four hours.

4. Immersion in a two per cent solution of carbolic acid for four hours. 
(b) Outer garments of wool or silk, and similar articles, which would be injured by immersion in boiling water or in a disinfecting solution:-

1. Exposure in a suitable apparatus to a current of steam for ten minutes.

2. Exposure to dry heat at a temperature of 110 degrees C. (230 degrees $\mathrm{F}$.) for two hours.

(c) Mattresses and blankets soiled by the discharges of the sick:-

1. Destruction by fire.

2. Exposure to superheated steam, 105 degrees C. (221 degrees F.), for ten minutes. (Mattresses to have the cover removed or freely exposed.)

3. Immersion in boiling water for half an hour.

Furniture and Articles of Wood, Leather, and Porcelain. Washing, several times repeated with:-

1. Solution of carbolic acid, two per cent.

For the Person. - The hands and general surface of the body of attendants of the sick, and of convalescents, should be washed with:-

1. Solution of chlorinated soda diluted with nine parts of water, $1: 10$.

2. Carbolic acid; two per cent solution.

3. Mercuric chlorid, $1: 1,000$.

For the Dead. - Envelop the body in a sheet thoroughly saturated with:-

1. Chlorid of lime in solution, four per cent,

2. Mercuric chlorid in solution, $1: 500$.

3. Carbolic acid in solution, five per cent.

For the Sick-Room.-(a) While occupied, wash all surfaces with:-

1. Mercuric chlorid in solution, $1: 1,000$.

2. Carbolic acid in solution, two per cent.

(b) When vacated, fumigate with sulphur dioxid for twelve hours, burning at least three pounds of sulphur for every thousand cubic feet of air space in the room ; then wash all surfaces with one of the abovementioned disinfecting solutions, and afterward with soap and hot water ; finally throw open doors and windows, and ventilate freely." 


\section{A P PEN D IX C.}

TABLE OF THE BONES.

HEAD (22) $\begin{cases}\text { Skull (8) } & \left\{\begin{array}{l}\text { Frontal (forehead). } \\ \text { 2 Temporal (temples). } \\ \text { 2 Parietal (side). } \\ \text { Occipital (posterior base). } \\ \text { Sphenoid (base). } \\ \text { Ethmoid (base of nose). }\end{array}\right. \\ & \left\{\begin{array}{l}\text { 2 Superior Maxilla (upper jaw). } \\ \text { 2 Nasal (bridge of nose). } \\ \text { 2 Malar (cheek). } \\ \text { 2 Lacrymal (corner of orbit). } \\ \text { 2 Turbinated (within nostrils). } \\ \text { 2 Palate (posterior hard palate). } \\ \text { Vomer (nasal partition). } \\ \text { Inferior Maxilla (lower jaw). }\end{array}\right.\end{cases}$

Cervical Region (8) $\quad\left\{\begin{array}{l}7 \text { Cervical Vertebræ (neck). } \\ \text { Hyoid Bone (base of tongue). }\end{array}\right.$

THoRAix (37)

(14 True, 6 False, 4 Floating Ribs. 12 Thoracic Vertebræ (back). Sternum.

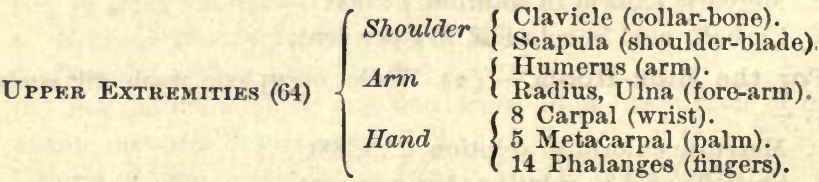

Lumbar Region (5) 5 Lumbar Vertebræ (loins).

Pelvis (4)

$\left\{\begin{array}{l}2 \text { Innominata. } \\ \text { Sacrum. } \\ \text { Coccyx. }\end{array}\right.$

Lower Extremities (60) $\begin{cases}\text { Thigh } & \begin{array}{l}\text { Femur. } \\ \text { Leg }\end{array} \\ & \left\{\begin{array}{l}\text { Patella (knee-pan). } \\ \text { Tibia (large bone). } \\ \text { Fibula (outer bone) }\end{array}\right. \\ \text { Foot } & \left\{\begin{array}{l}7 \text { Tarsal (instep, heel) } \\ 5 \text { Metatarsal (arch). } \\ \text { 14 Phalanges (toes). }\end{array}\right.\end{cases}$ 
Daily Excretions. - Sweat, from 1.5 lbs. to 4.5 lbs.; urea, about 1 oz. ; organic matter exhaled, 3 grains ; urine, $53 \mathrm{oz}$.

"Of the entire excreta, 32 per cent pass off by the breath; 17 per cent by the skin; 46.5 per cent by the kidneys; 4.5 per cent by the alimentary canal." - CUTTER.

Number of Sweat Glands. - The number of sweat glands may be as high as 3,500 in a square inch, and the average is estimated at 2,800 per square inch; as there are about 2,500 square inches of body surface, it is readily computed that there are several millions of sweat glands.

Number of Hairs on the Human Head. - The average number of hairs on the head is 120,000 . They are set obliquely, and are controlled by muscles so that they may be made to stand erect, or nearly so, under the influence of certain emotions, as fear, anger, etc.

Huxley and others have classified the races of men according to the hair, into the Ulotrichi, or crisp or woolly haired division, including the negroes, bushmen, etc. ; and Leiotrichi, or smooth-haired, subdivided into the Australioid, the Mongoloid, the Xanthochroic, and the Melanochroic.

In Europeans the hair is oval in cross-section; in the Japanese and Chinese it is circular.

Circulation. - Rate of blood flow : in the large arteries, from 12 to 16 inches a second; in the caval veins, about 4 inches a second; in the capillaries, from 1 inch to 1.5 inches a minute. A portion of the blood makes the complete circulation (in a horse) in less than half a minute. This is found by putting some readily detected chemical into one jugular vein, and noting how soon it appears in the other jugular vein. The time necessary for all the blood to pass through the heart is estimated as follows : Each ventricle pumps about six ounces of blood at each stroke. At this rate thirty strokes, 25 to 50 seconds (or less), would have pumped all the blood in the body. Still, some of the blood (from the shorter circuits) may have been pumped twice, and some (from the longer routes) may not yet have been around once. And since the total amount of blood has been only approximately determined, these figures are not very accurate.

Number of blood corpuscles to the cubic inch, about $83,000,000$.

Dr. Tanner's Forty Days' Fast (Newspaper Account). No Food but Water Taken. - When Dr. Tanner came to New York from Minnesota he weighed 184 pounds. He was six weeks making ar 
rangements for his fast; and when he began his experiment his weight was $157 \frac{1}{2}$ pounds. He weighed $121 \frac{1}{2}$ pounds on the day his fast ended. He had therefore lost $62 \frac{1}{2}$ pounds since he came to the city, and 36 pounds since he began his fast. Dr. Hammond, the well-known New York physician whose assertion that a forty days' fast was a physical impossibility led Dr. Tanner to make the attempt, came out in a card in the New York papers declaring that he believed the fast had been fairly conducted.

On each day of his fast Dr. Tanner weighed as follows :-

\begin{tabular}{|c|c|c|c|c|c|c|c|c|c|c|c|c|c|c|c|c|c|c|c|c|c|}
\hline $\mathbf{A Y}$. & & & & & & & & & & OUADS. & DAY. & & & & & & & & & & \\
\hline 1st & - & & - & • & • & - & - & - & - & & & - & & - & & & - & • & - & - & - $131 \frac{1}{2}$ \\
\hline $3 d$ & - & • & - & - & & 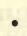 & • & - & • & 153 & 26 th & - & & • & & & & - & • & - & $131_{2} \frac{1}{2}$ \\
\hline 5th & - & - & - & • & - & - & - & • & • & - $147 \frac{1}{2}$ & 27 th & - & & - & & & • & • & - & • & $130 \frac{1}{2}$ \\
\hline 7 th & - & - & - & • & - & & 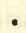 & & - & - $143 \frac{1}{2}$ & $28 \mathrm{th}$ & . & & • & & & & . & - & - & 1293 \\
\hline 11th & - & - & - & - & - & - & - & • & - & - 1394 & 29th & - & & - & & & • & • & $\bullet$ & - & - \\
\hline 13th & - & - & • & - & - & • & - & . & - & - 1364 & 30th & . & & - & & & - & • & . & & 130 \\
\hline 14th & • & - & - & - & - & - & - & • & - & - 133 & 31st & - & & - & & & • & - & & - & 128 \\
\hline 16th & - & - & - & - & • & - & - & • & - & - 132 & $32 d$ & $0^{\circ}$ & & - & & & & & - & - & - $127 \frac{1}{2}$ \\
\hline 17th & (8. & .30 & P.I & M.) & - & . & - & . & - & - $133 \frac{1}{2}$ & 33d & . & & • & & & • & • & & & $126 \frac{1}{2}$ \\
\hline 17 th & (11 & & M. & & • & - & . & . & - & - $135 \frac{1}{2}$ & 34 th & - & & - & & & - & . & • & - & - $126 \frac{\alpha}{2}$ \\
\hline 18th & - & • & . & - & - & - & - & - & - & - $136 \frac{1}{2}$ & 35th & - & & - & & & - & & • & & \\
\hline 19th & . & • & - & - & - & - & - & • & - & - 136 & 36th & - & & - & & & • & - & - & & \\
\hline 20 th & $(4$ & P.N & M.) & . & - & - & - & • & - & - $135 \frac{1}{2}$ & 37 th & . & & & & & & • & • & & - $125 \frac{1}{2}$ \\
\hline 20 th & $(5$ & A.I & M.) & . & - & • & • & • & • & - 135 & 38th & - & & - & & & & • & • & - & \\
\hline 21st & - & - & - & - & - & • & • & - & • & - 135 & 39 th & & & & & - & & & & & - $122 \frac{1}{2}$ \\
\hline $22 \mathrm{~d}$ & - & • & • & . & & • & & & • & - $133 \frac{1}{2}$ & 40 th & & & & & - & • & • & - & - & - $121 \frac{1}{2}$ \\
\hline 6 th & - & • & • & • & & & & & & - 132 & & & & & & & & & & & \\
\hline
\end{tabular}

Cavities of the Body.-1. Mucous cavities (open to the external air). Digestive tube, respiratory passages, genito-urinary passages, external and middle ear, etc.

2. Serous cavities (closed). They may all be said to be lymph cavities. They are the lymph spaces throughout the body, and the large spaces, called the pleural cavity around the lungs, the pericardial cavity around the heart, the peritoneal cavity in the abdomen, the arachnoid cavity around the brain, and a similar one along the spinal cord.

3. Synovial cavities in the joints.

4. Blood cavities, - the inside of the heart and blood tubes.

5. Secretion cavities, - the cavities and tubes from the glands; for example, the bile sac and its duct.

6. Bone cavities. 


\section{LOSSES OF THE TISSUES DURING STARVATION.}

\section{(FROM EXPERIMENT ON A CAT.)}

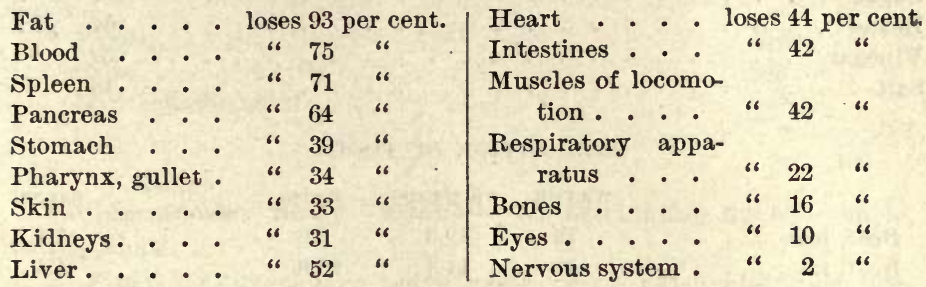

QUANTITY OF WATER IN 1,000 PARTS.

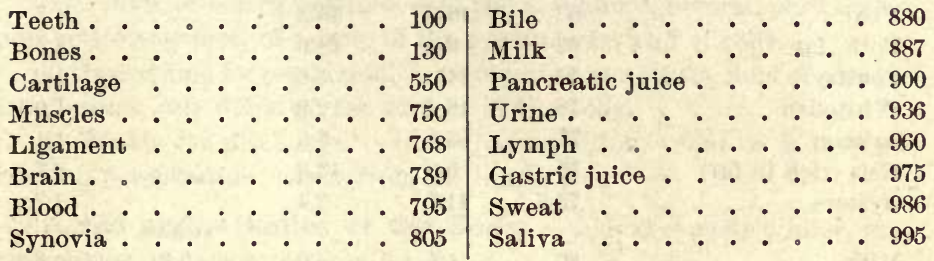

THE LOSS OF WATER FROM THE BODY.

From the Alimentary canal (feces) . . . . . . . 4 per cent.

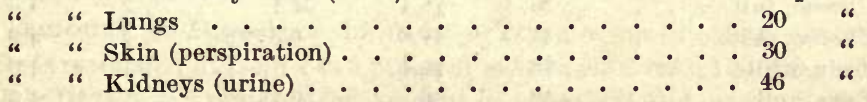

ELEMENTS IN THE HUMAN BODY.

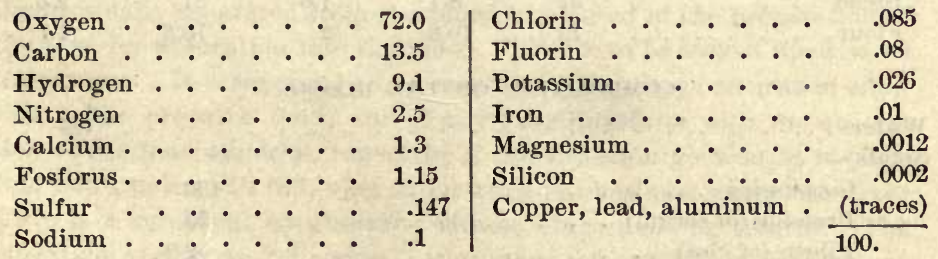

DAILY RATION OF A U. S. SOLDIER DURING THE LATE WAR.

Bread or flour . . . . . . . . . . . . . . 22 oz.

Fresh or salt beef (or pork or bacon $12 \mathrm{oz}$.) - . . . . . . 20 "

Potatoes (three times a week) . . . . . . . . . . 16 " 


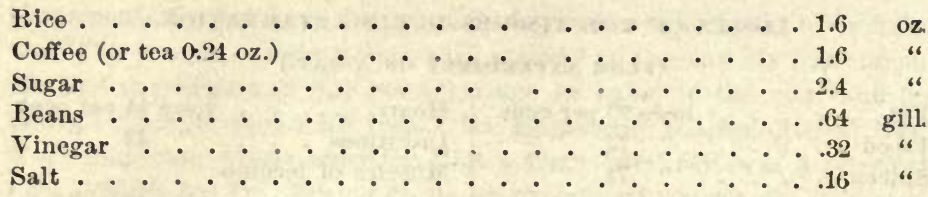

COMPOSITION OF FOODS.

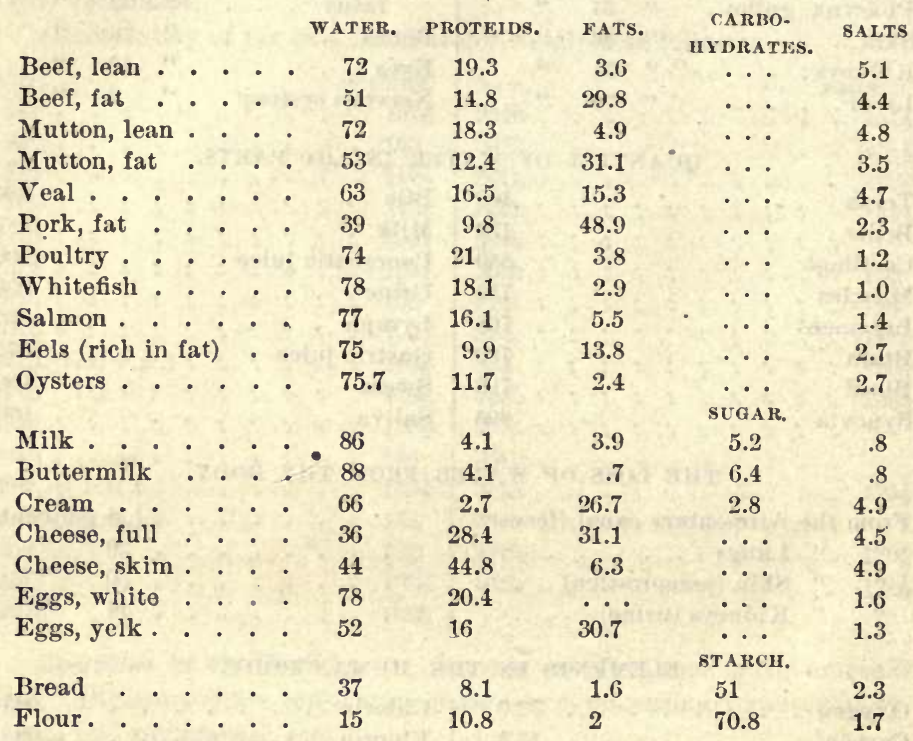

COMPOSITION OF THE BLOOD.

Water .

Solids -

Corpuscles . . . . . . . . . . . 130

Proteids (of serum) . . . . . . . 70

Fibrin (of clot) . . . . . . . . . . 2.2

Fatty matters (of serum) . . . . . . . . 1.4

Inorganic salts . . . . . . . . . . 6.0

Gases, urea, kreatin, etc. . . . . . . . . 6.4 


\section{COMPOSITION OF GASTRIC JUICE.}

Water

99.44

Solids -

Pepsin, . . . . . . . . . . . . . .319

Salts . . . . . . . . . . . . .218

Hydrochloric acid . . . . . . . . . . . . . .

Fluids of the Body (FonD). - 1. Circulating fluids, - chyle, lymph, blood.

2. Fluids for digestion, - saliva, gastric juice, pancreatic juice, bile, intestinal juice.

3. Fluids of closed cavities, - of the arachnoid, pleural, pericardial, and peritoneal sacs, of joints, of the eye and ear, and of cells.

4. Secretions for protection, - cerumen or wax, tears, fluid of mucous membranes, oily fluids on the surface of the body.

5. Fluids for discharge, - intestinal secretion, renal or kidney secretion, perspiration, vapor from the lungs, etc.

Acids and Alkalies of the Body.-Acids, - gastric juice, mucus, chyme, contents of large intestine.

Alkalies, - salira (or neutral), pancreatic juice, intestinal juice, bile (or neutral), contents of small intestine, sweat.

Amount of Digestive Liquids. - The amount of saliva secreted daily is estimated at from 1 to 3 pints, of gastric juice from 10 to 20 pints, of bile from 2 to 3 pints. The amount of intestinal and other juices is difficult to estimate. But it is readily seen that a very large amount of liquid is daily separated from the blood to be used in the preparation of the food for absorption into the blood. This is to be looked upon as an investment. It is supposed to be reabsorbed with large returns in addition to the prepared food; and if anything interferes with the absorption of the food material, especially if the secretion goes on, it is plain that bankruptcy will follow as surely as in the business world whenever there is a continual expenditure without corresponding returns: The condition known as "diarrhea" illustrates this condition, perhaps, as well as any well-known condition of the body.

Specific Gravity of the Liquids of the Body. - As all the liquids of the body have dissolved and suspended in them various salts and other matters, they are all heavier than water. 
TIME REQUIRED FOR DIGESTION OF FOODS (BEAUMONT).

\begin{tabular}{|c|c|c|c|c|c|c|c|c|}
\hline & & & & & & & & \\
\hline Pigs' feet, soused (boiled & & 1 & 00 & Mutton (roasted) & - & - & & 15 \\
\hline Tripe, soused (boiled). & & 1 & 00 & Eggs (hard boiled) . & - & . & & 30 \\
\hline oup, barley (boiled) . & & 1 & 30 & Eggs (fried) . . & . & . & & 30 \\
\hline rout, salmon, fresh (fri & & 1 & 30 & Potatoes, Irish (boil & led) & - & & 30 \\
\hline Venison steak (broiled) & .. & 1 & 35 & Oysters (stewed) . & - $\cdot$ & - & & 30 \\
\hline k (boiled) . . . . & . & 2 & 00 & Beets (boiled) . & - & • & & 45 \\
\hline bage, with vinegar ( $\mathrm{r}$ & & 2 & 00 & Green corn and beans & s (boi & iled & & 45 \\
\hline $\operatorname{resh}(\mathrm{raw})$ & - . & 2 & 00 & Salmon (boiled). . & & - & & 00 \\
\hline Apples, sour, mellow (ra & & - 2 & 00 & Soup, beef, vegetal & bles, & & & \\
\hline Milk (raw). . . . & & 2 & 15 & bread (boiled) & $x^{\circ}$ & - & & 00 \\
\hline Curkey (roasted) & & 2 & 30 & rn-yard (ro & & d) & & 00 \\
\hline Eggs, fresh (soft boiled) & & 3 & 00 & (fried & & $\cdot$ & & 00 \\
\hline Beefsteak (broiled) . & & 3 & 00 & Pork, salt (fried). & & . & & 15 \\
\hline , fresh (boiled) & & - 3 & 00 & Veal (fried) . & & • & & 30 \\
\hline , chicken (boiled) & - & - 3 & 00 & Cabbage (boiled) . & • & - & & 30 \\
\hline orn (baked) . & & - 3 & 15 & Duck, wild (roasted & & $\bullet$ & & 30 \\
\hline Oysters, fresh (roasted) & & - 3 & 15 & Pork, fresh (roasted & & & & 15 \\
\hline
\end{tabular}

Tissues of the Body (Ford.) - 1. Osseous, - bones and teeth.

2. Cartilaginous, - pure cartilage and fibro-cartilage.

3. Fibrous, - white and yellow ; i.e., the white firm and strong to bind parts together, and the yellow elastic to afford elasticity and freedom of movement.

4. Muscular, - striated or voluntary muscles, and non-striated or involuntary.

5. Adipose, - fat cells and inclosed fat.

6. Epithelial, - epithelium, epidermis, hair, and nails.

7. Nervous, - cerebro-spinal and sympathetic.

WEIGHT OF THE PARTS OF THE BODY (HUXLEY).

(OF A MAN WEIGHING 154 LBS.)

Muscles and their appurtenances. . . . . . . . $68 \mathrm{lbs}$.

Skeleton.

Skin

Fat.

Brain .

Thoracic viscera (heart $10 \mathrm{oz}$.$) .$

Abdominal viscera (liver $4 \mathrm{lbs}$.)

Blood (that can be withdrawn) 10.5 “ 28 "

3 “

2.5 “

. 11

$7 \%$ 
DAILY INCOME AND EXPENDITURE OF THE BODY. INCOME.

Solid food . . . . 8,000 grains

Water . . . . 37,650

Oxygen . . . . 13,000

$$
\overline{58,650}
$$

(3,308 grams, or about 8.33 lbs.)
EXPENDITURE.

Lungs . . . . 20,000 grains

Skin. . . . . 11,750 "

Kidneys . . . . 24,100 "

Intestines . . . 2,800 "

MEASUREMENTS OF TISSUES (KIRKE).

AVERAGE SIZE IN FRACTIONS OF AN INCH.

\begin{tabular}{|c|c|c|}
\hline & WIDTH. & LENGTH. \\
\hline Air cells. . . . . . . . & $\frac{1}{70}$ to $\frac{1}{50}$ & \\
\hline Blood cells, red . . . . & $3 \frac{1}{200}$ & Thickness. $10 \frac{1}{0} 00$ \\
\hline Blood cells, colorless . . . & 2500 & \\
\hline Capillaries . . . . . . & $30 \frac{1}{300}$ to $1 \frac{1}{200}$ & \\
\hline Cilia . . • . . . . . . & & 5000 to 2500 \\
\hline Cones of retina at yellow spot & $T_{2} \frac{1}{000}$ to $T_{0000}$ & \\
\hline Fat cells: . . . : . & $5 \frac{1}{500}$ to $\frac{1}{400}$ & \\
\hline Gastric glands . . . . & 500 to $\frac{1}{360}$ & $\frac{1}{60}$ to $\frac{1}{20}$ \\
\hline Muscle fiber, striated . . . & $3 \frac{1}{0} 0$ to $\frac{1}{4} \frac{1}{0}$ & $1 \frac{1}{2}$ inches. \\
\hline Muscle fiber, plain . . . & $4 \frac{1}{500}$ to $\frac{1}{3500}$ & $6 \frac{1}{00}$ to $\frac{1}{400}$ \\
\hline Nerve cells, brain . . . & $3 \frac{1}{000}$ to $\frac{1}{300}$ & \\
\hline Nerve fibers, medullated. & $12 \frac{1}{200}$ to 1500 & \\
\hline Nerve fibers, non-medullated & $8 \frac{1}{000}$ to 50100 & \\
\hline Papillæ of skin . . . . . & $\frac{1}{200}$ to ${ }_{1}^{\frac{1}{0}} 0$ & \\
\hline Villi . . . . . . . . & $\frac{1}{2} \frac{1}{0}$ to $\frac{1}{70}$ & $\frac{1}{50}$ to $\frac{1}{8}$ \\
\hline
\end{tabular}

The size and various pigmentations of the brain are finely shown in the following:

$2 \%$ of formaldehyde ( $=5 \%$ formalin).$\quad .89$

$95 \%$ alcohol . . . . . . . . . 10

sodium ehlorid . . . . . . . 1 


\section{TO SHOW THE ACTION OF CILIA.}

Kill a frog, and destroy the brain and spinal cord as directed on p. 37 . Lay the frog on its back ; divide the lower jaw longitudinally, and continue the cut as far as the stomach. Pin the flaps out to the sides, thus laying bare the roof of the mouth and gullet. If the lining of the mouth seems dry, moisten it with "saline solution." Cut some cork fine, like sawdust, and drop a little of it on the roof of the mouth between the eyes. The current of mucus, caused by the vibration of the cilia, carries the particles toward the stomach. Snip off a little of this ciliated lining and mount in saline solution, and examine under a high power of a good microscope. Or snip off a little of the margin, or scrape a little off the surface of the gill of a clam, and examine as above. 


\section{GLOS S A RY.}

Albumen ( $\left.a l-b \bar{u}^{\prime} \cdot m e n\right)$. The white of an egg.

Albumin ( $\left.a l-b \bar{u}^{\prime}-\min \right)$. A proteid substance, the clief constituent of the body. Its molecule is highly complex, and varies widely within certain limits in different organs and in different conditions.

Albuminuria ( $\left.a l-b \bar{u}^{\prime}-m i-n \bar{u}^{\prime}-r i-a\right)$. The presence of albumin in the urine, indicating changes in the blood or in the kidneys.

Amylopsin (am-i-lop'-sin). A ferment said to exist in pancreatin.

Anabolism ( $a$ - $\left.a b^{\prime}-o-l i z m\right)$. Synthetic or constructive metabolism. Activity and repair of function; opposed to katabolism.

Arbor Vitae ( $a r^{\prime}$-bor $\left.v \bar{\imath}^{\prime}-t \bar{e}\right)$. A term applied to the branched appearance of a section of the cerebellum.

Argon ( $\left.a r^{\prime}-g o n\right)$. A newly discovered element similar to nitrogen (found in the air).

Arytenoid ( $\left.a r-i-t \bar{e}^{\prime}-n o i d\right)$. Resembling the mouth of a pitcher, as the arytenoid cartilages of the larynx.

Atlas ( $\left.a t^{\prime}-l a s\right)$. The uppermost of the cervical vertebrae (from the mythical Atlas who supported the Earth).

Auricle $\left(a w^{\prime}-r i-k l\right)$. The auricles of the heart are the two cavities between the veins and the ventricles. Also, the pinna and external meatus of the ear.

Axis ( $\left.a k^{\prime}-s i s\right)$. The second cervical vertebra, on which the head, with the atlas, turns.

Bacterium (bak-tét-ri-um), pl. bacteria. A genus of microscopic fungi characterized by short, linear, inflexible, rod-like forms - without tendency to unite into chains or filaments.

Biceps ( $b \bar{\imath}^{\prime}$-seps). Biceps brachii, the flexor of the arm.

Bicuspid (bi-kus'-pid). Having two points ; the bicuspid or premolar teeth; the bicuspid valve, between the left auricle and the left ventricle.

Brachial (bra $\bar{a}^{\prime}-k e-a l$ or $\left.b r a k^{\prime}-i-a l\right)$. Pertaining to the arm. 
Bronchus (brong'-kus), pl. bronchi. 'The two tubes into which the trachea divides opposite the third thoracic vertebra, called respectively the right and left bronchus.

Caffein $\left(k a f^{\prime}-\bar{e}-i n\right)$. An alkaloid that occurs in the leaves and beans of the coffee-tree, in Paraguay tea, etc.

Canaliculus (kan-a-lik'-u-lus),pl. canaliculi. The crevices extending from lacunae, through which nutrition is conveyed to all parts of the bone.

Canine $\left(k a-n i n^{\prime}\right.$ or $\left.k \bar{a}^{\prime}-n \bar{i} n\right)$. The conical teeth between the incisors and the premolars.

Capillary (kap'-i-lā-ri or $\left.k a-p i l^{\prime}-a-r i\right)$. A minute blood-tube connecting the smallest ramification of the arteries with those of the veins.

Capsule $\left(k a p^{\prime}-s \bar{u} l\right)$. A tunic or bag that incloses a part of the body or an organ.

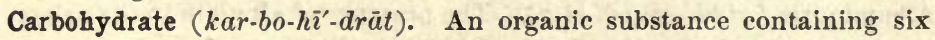
carbon atoms or some multiple of six, and hydrogen and oxygen in the proportion in which they form water; that is, twice as many hydrogen as oxygen atoms. Starches, sugars, and gums are carbohydrates.

Cardiac (kär'-di-ak). Pertaining to the heart.

Carotid (ka-rot'-id). The principal right and left arteries of the neck.

Carpus (kär'-pus). Belonging to the wrist; as the carpal bones.

Cartilage ( $\left.k \ddot{a} r^{\prime}-t i-l a \bar{j} j\right)$. Gristle of various kinds, articular, etc.

Casein $\left(k \bar{a}^{\prime}-s e-i n\right)$. A derived albumin, the chief proteid of milk, precipitated by acids and by rennet at $40^{\circ} \mathrm{C}$.

Cecum $(s \bar{e}-k u m)$. The large blind pouch or cul-de-sac, in which the large intestine begins.

Centrum (sen'-trum). The center or middle part ; the body of a vertebra, exclusive of the bases of the neural arches.

Cerebellum (ser-e-ebl'-um). . The inferior part of the brain, lying below the cerebrum.

Cerebrum (ser'-e-brum). The chief portion of the brain, occupying the whole upper part of the cranium.

Cervical (ser'-vi-kal). Pertaining to the neck, as cervical vertebrae.

Chordae tendineae $\left(k o r^{\prime}-d \bar{e}\right)$. The tendinous cords connecting the fleslyy columns of the heart with the aurlculo-ventricular valves.

Choroid ( $k \bar{o}^{\prime}$-roid). The second or vascular coat of the eye, continuous with the iris in front, and lying between the sclerotic and the retina. 
Chyle (kill). The milk-white fluid absorbed by the lacteals during digestion.

Chyme (kim). Food that has undergone gastric digestion, and has not yet been acted upon by the biliary, pancreatic, and intestinal secretions.

Cilium (sil'-i-um), pl. ciliä. The eyelashes; also the hair-like appendages of certain epithelial cells, whose function is to propel fluid or particles along the passages that they line.

Ciliary (sil'-i-a-ri). Pertaining to the eyelid or eyelash; also by extension to the ciliary apparatus or the structure related to the mechanism of accommodation. Pertaining to the cilia.

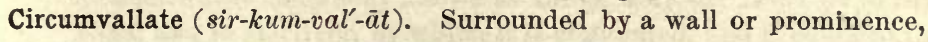
as the circumvallate papillae on the tongue.

Clavicle $\left(k l a v^{\prime}-i-k l\right)$. The collar-bone.

Coccyx (kok'siks). The last bone of the spinal column, formed by the union of four rudimentary vertebrae.

Cochlea $\left(k o k^{\prime}-l \bar{e}-a\right)$. A cavity of the internal ear, resembling a snailshell.

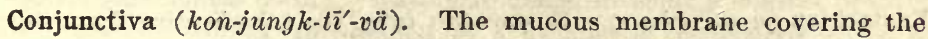
anterior portion of the globe of the eye, reflected on, and extending to, the free edge of the lids.

Corpus Arantii (kor'-pus). The tubercles, one in the center of each segment of the semilunar valves.

Corpuscle (kor'-pus-l). A name loosely applied to almost any small, rounded or oval body, as the blood corpuscles.

Cortex (kor'-teks). Bark. The outer layer of gray matter of the brain ; the outer layer, cortical substance, of the kidney.

Cricoid ( $\left.k r \bar{\imath}^{\prime}-k o i d\right)$. Ring-shaped, as the cricoid cartilage of the larynx.

Dentine (den'-tin). The ivory-like substance constituting the bulk of the tooth, lying under the enamel of the crown and the cement of the root.

Diabetes ( $\left.d \bar{\imath}-a-b \bar{e}^{\prime}-t \bar{e} z\right)$. The name of two different affections, diabetes mellitus, or persistent glycosuria, and diabetes insipidus, or polyuria, both characterized, in ordinary cases, by an abnormally large discharge of urine. The former is distinguished by the presence of an excessive quantity of sugar in the urine.

Dialysis ( $\left.d \bar{i}-a l^{\prime}-i-s i s\right)$. The operation of separating crystalline from colloid substances by means of a porous diaphragm, the former 
passing through the diaphragm into the pure water upon which the dialyzer rests.

Digastric (di-gas'-trik). Having two bellies, as the digastric muscle, enlarged near each end and with a tendon in the middle.

Duodenum ( $\left.d \bar{u}-\bar{o}-d \bar{e}^{\prime}-n u m\right)$. The first part of the small intestine, beginning with the pylorus.

Emulsion ( $\bar{e}-m u l^{\prime}-$ shun). Water or other liquid in which oil, in minute subdivision of its particles, is suspended.

Enamel (en-am'-el). The hard covering of the crown of a tooth.

Endothelium (en-do-the'-li-um). The internal lining membrane of serous, synovial, and other internal surfaces, the homolog of epithelium.

Enzyme (en'-zim). Any chemic or hydrolytic ferment, as distinguished from organized ferments such as yeast; unorganized ferment.

Epiglottis (ep-i-glot'-is). A thin fibro-cartilaginous valve that aids in preventing food and drink from passing into the larynx.

Esophagus ( $\left.\bar{e}-s o f^{\prime}-a-g u s\right)$. The musculo-membranous tube extending from the pharynx to the stomach.

Eustachian ( $\left.u-s t \bar{a}^{\prime}-k i-a n\right)$. Eustachian tube, the tube leading from the middle ear to the pharynx.

Facet (fas'-et). A small plane surface. The articulating surface of a bone.

Femur $\left(f \bar{e}^{\prime}-m e r\right)$. The thigh-bone.

Ferment (fer'-ment). Any micro-organism, proteid, or other chemic substance capable of producing fermentation, i.e., the oxidation and disorganization of the carbohydrates.

Fibrin ( $\bar{f}^{\prime}$-brin). A native albumen or proteid, a substance that, becoming solid in shed blood, plasma, and lymph, causes coagulation of these fluids.

Fibula $\left(f i b^{\prime}-u-l \ddot{a}\right)$. The smaller or splint bone in the outer part of the leg, articulating above with the tibia. and below with the astragalus and tibia.

Filiform ( $f l^{\prime}-i$-form). Thread-like, as the filiform papillae.

Frontal (fron'-tal). Belonging to the front, as the frontal bone.

Fungiform (fun'-ji-form). Having the form of a mushroom, as fungiform papillae.

Ganglion (gang'-gli-on), pl. ganglions or gangliä. A separate and semiindependent nervous center, communicating with other ganglia or nerves, with the central nervous system, and peripheral organs. 
Gastric (gas'-trik). Pertaining to the stomach.

Gelatin (jel'-a-tin). An albuminoid substance of jelly-like consistence, obtained by boiling skin, connective tissue, and bones of animals in water. The glue of commerce is an impure variety.

Glosso-pharyngeal ( $\left.g l o s^{\prime}-o-f a-r i n^{\prime}-j e-a l\right)$. Pertaining to the tongue and larynx.

Gluten (glö'ten). A substance resembling albumin, and with which it is probably identified ; it occurs abundantly in the seeds of cereals.

Glycogen ( $\left.g l \bar{\imath}^{\prime}-k o-j e n\right)$. A white amorphous powder, tasteless and odorless, forming an opalescent solution with water, and insoluble in alcohol. It is commonly known as animal starch. It occurs in the blood and in the liver, by which it is elaborated, and is changed by diastasic ferments into glucose.

Gustatory ( $\left.g u s^{\prime}-t \bar{a}-t o-r i\right)$. Pertaining to the special sense of taste and its organs.

Hashish (hash'-èsh). A preparation from Indian hemp, Cannabis indica. It is a powerful narcotic.

Haversian (ha-ver'-zian). Haversian canal, in bone, a central opening for blood-tubes, surrounded by a number of concentric rings, or lamellae, of bone.

Hemoglobin (hem-ō-glö-bin). A substance existing in the corpuscles of the blood, and to which their red color is due.

Hepatic $\left(h \bar{e}-p a t^{\prime}-i k\right)$. Pertaining or belonging to the liver.

Hilum ( $h \bar{\imath}^{\prime}-l u m$ ). A small pit, scar, or opening in an organic structure ; the notch on the internal or concave border of the kidney.

Humerus ( $\left.h \bar{u}^{\prime}-m e-r u s\right)$. The bone of the upper arm.

Humor ( $\left.h \bar{u}^{\prime}-m o r\right)$. Any liquid, or semi-liquid, part of the body.

Hyoid ( $h \bar{i}^{\prime}$-oid). Having the form of the letter $U$. The hyoid bone situated between the root of the tongue and the larynx, supporting the tongue and giving attachment to its muscles.

Hypo-glossal ( $\left.h \bar{i}-p \bar{o}-g l o s^{\prime}-a l\right)$. Under the tongue.

Iliac $\left(i l^{\prime}-i-a k\right)$. Pertaining to the ilium, or region of the flanks, as iliac artery, vein, etc.

Incisor ( $i n-s \bar{i}^{\prime}$-sor). The chisel-shaped front teeth.

Inhibition (in-hi-bish'-un). The act of checking, restraining, or suppressing ; any influence that controls, retards, or restrains. Inhibitory nerves and centers are those intermediating a modification, stoppage, or suppression of a motor or secretory act already in progress. 
Innominate (i-nom'-i-näte). Nameless; a term applied to several parts of the body to which no other definite name has been given, as the innominate bone, artery, vein, etc.

Invertin (in'-ver-tin). $\mathbf{\Lambda}$ ferment found in the intestinal juice, and also produced by several species of plants; it converts cane-sugar in solution into invert sugar.

Jugular $\left(j \ddot{o}^{\prime}-g \bar{u}-l a ̈ r\right)$. Pertaining to the throat, as the jugular vein.

Katabolism (ka-tab'-ö-lizm). Analytic or destructive metabolism; a physiologic disintegration; opposed to anabolism.

Lacrymal (lak'-ri-mal). Having relation to the organs of the secretion, transfer, or excretion of tears.

Lacuna $\left(l \bar{a}-k \bar{u}^{\prime} \cdot n \ddot{a}\right)$. A little hollow space ; especially the microscopic cavities in bone occupied by the bone corpuscles, and communicating with one another and with the haversian canals and the surfaces of the bone through the canaliculi.

Lamella (lā-mel'-ä), pl. lamellae. A thin lamina, scale, or plate; of bone, the concentric rings surrounding the haversian canals.

Larynx (lar'-ingks). The upper part of the air passage between the trachea and the base of the tongue; the voice-box.

Legumin (lé-gu'-min). A proteid compound in the seeds of many plants belonging to the natural order Leguminosae (peas, beans, lentils, etc.).

Lumbar (lum'-bär), pertaining to the loins, especially to the region about the loins.

Lymphatic (lim-fat'-ik). Pertaining to lymph.

Lymphatics (lim-fat'-iks). The tubes that convey lymph.

Lymphatic glands. The glands intercalated in the pathway of the lymphatic tubes, through which lymph is filtered.

Massage $\left(m a-s \ddot{a} z h^{\prime}\right)$. A method of effecting changes in the local and general nutrition, action and other functions of the body, by rubbing, kneading, and other manipulation of the superficial parts of the body by the hand or an instrument.

Masseter (mas'-e-ter). A chewing-muscle felt on the angle of the jaw.

Medullary (med'-u-lā-ri). Pertaining to the medulla, or marrow ; resembling marrow. Also pertaining to the white substance of the brain contained within the cortical envelop of gray matter.

Mesenteric (mez-en-ter'-ik). Pertaining to the mesentery, as artery, vein, etc. 
Mesentery (mez'-en-ter-i). A fold of the peritoneum that connects certain portions of the intestine with the dorsal abdominal wall.

Metabolism (me-tab'-o-lizm). A change in the intimate condition of cells ; (1) constructive or synthetic metabolism is called Anabolism ; in anabolism, the substance is becoming more complex and is accumulating force; (2) destructive or analytic metabolism is called Katabolism ; in katabolism there is disintegration, the material is becoming less complex, and there is loss or expenditure of force.

Metacarpus (met-a-kär'-pus). The bones of the palm of the hand.

Metatarsus (met-a-tär'-sus). The five bones of the arch of the foot, situated between the tarsus and the phalanges.

Mitral ( $m \bar{\imath}^{\prime}$-tral). Resembling a miter; mitral valve, with two flaps, between the left auricle and the left ventricle.

Molar $\left(m \bar{o}^{\prime}\right.$-lär $)$. Mill; the grinding-teeth.

Mucous ( $\left.m \bar{u}^{\prime}-k u s\right)$. A term applied to those tissues that secrete mucus.

Mucus ( $\left.m \bar{u}^{\prime}-k u s\right)$. A viscid liquid secretion of mucous membranes, composed essentially of mucin, holding in suspension desquamated epithelial cells, etc.

Myosin ( $\left.m \bar{\imath}^{\prime}-0-\sin \right)$. A proteid of the globulin class, - the chief proteid of muscle. Its coagulation after death causes rigor mortis.

Narcosis (när-kón-sis). The deadening of pain, or production of incomplete or complete anesthesia by the use of narcotic agents, such as anesthetics, opium, and other drugs.

Narcotic (när-kot'-ic). A drug that produces narcosis.

Neural $\left(n \bar{u}^{\prime}-r a l\right)$. Pertaining to the nerves.

Neuroglia $\left(n \bar{u}-r_{0} g^{\prime}-l i-\ddot{a}\right)$. The reticulated framework or skeleton-work of the substance of the brain and spinal cord. The term is sometimes abbreviated to glia.

Nucleus $\left(n \bar{u}^{\prime}-k l \bar{e}-u s\right)$. The essential part of a typical cell, usually round in outline, and situated in the center.

Occipital (ok-sip'-i-tal). Pertaining to the occiput or back part of the head, as the occipital bone.

Odontoid (o-don'-toid). Resembling a tooth; the tooth-like process (axis) of the second cervical vertebra, on which the atlas turns. olfactory (ol-fak'-tō-ri). Pertaining to the sense of smell.

Osmosis $\left(o s-m \bar{o}^{\prime}-s i s\right)$. That property by which liquids and crystalline substances in solution pass through porous septa; endosmosis and exosmosis. 
0xy-hemoglobin (ok-si-hem-ō-gló-bin). Hemoglobin united, molecule for molecule, with oxygen. It is the characteristic constituent of the red corpuscles to which the scarlet color of arterial blood is due.

Pancreas (pan'-krē-as). A large racemose gland lying transversely across the dorsal wall of the abdomen. It secretes a clear liquid for the digestion of proteids, fats, and carbohydrates. The sweetbread of animals, vulgarly called the "belly sweet-bread" in contra-distinction to the thymus, or true sweet-bread.

Pancreatin (pan'-krē- $a$-tin). The active element of the pancreatic juice. Papilla ( $\left.p \bar{a}-p i l^{\prime}-\ddot{a}\right)$, pl. papillae. Any soft, conical elevation, as papillae of the dermis, tongue, etc.

Papillary ( $\left.p a p^{\prime}-i-l \bar{a}-r i\right)$. Pertaining to a papilla; papillary muscles, the conic muscular columns of the heart, to which the chordae tendineae are attached.

Parietal ( $\left.p \bar{a}-r \bar{\imath}^{\prime}-e-t a l\right)$. Pertaining to the walls, as the parietal bone.

Parotid ( $\left.p a-r o t^{\prime}-i d\right)$. Near the ear, as the parotid salivary glands.

Patella $\left(p a-t e l^{\prime}-a\right)$. The knee-pan.

Peptone $\left(p e p^{\prime}-t_{0} n\right)$. A proteid body produced by the action of peptic and pancreatic digestion.

Pericardium (per-i-kär'-di-um). The closed membranous sac or covering that envelops the heart.

Periosteum (per-i-os'-tē-um). A fibrous membrane that invests the surfaces of the bones, except at the points of tendinous and ligamentary attachments, and on the articular surfaces where cartilage is substituted.

Peristaltic (per-i-stal'-tik). The peculiar movement of the intestine and other tubular organs, consisting in a vermicular shortening and narrowing of the tube, thus propelling the contents onward. It is due to the successive contractions of the bundles of longitudinal and circular muscular fibers.

Peritoneal (per-i-tō-ne'-al). Pertaining to the peritoneum.

Peritoneum ( $\left.p e r-i-t \bar{o}-n e^{\prime}-u m\right)$. The serous membrane lining the interior of the abdominal cavity, and surrounding the contained viscera. The peritoneum forms a closed sac, but is rendered complex in its arrangement by numerous foldings produced by its reflection upon the viscera.

Phalanges ( $\left.f \bar{a}-l a n^{\prime}-j e \bar{e} z\right)$, plural of phalanx ( $\left.f \bar{a}^{\prime}-l a n g k s\right)$. Any one of the bones of the fingers or toes. 
Pharynx (far'-ingks). The cavity back of the soft palate. It communicates anteriorly with the posterior nares, laterally with the eustachian tubes, ventrally with the mouth, and posteriorly with the gullet and larynx.

Plasma $\left(p l a z^{\prime}-m \ddot{a}\right)$. The original undifferentiated substance of nascent, living matter. The fluid part of the blood and lymph.

Pleura $\left(p l \ddot{o}^{\prime}-r \ddot{a}\right)$. The serous membrane which envelops the lungs, and which, being reflected back, lines the inner surface of the thorax.

Plexus (plek'-sus). An aggregation of vessels or nerves forming an intricate net-work.

Pneumogastric (nu-mō-gas'-trik). Pertaining conjointly to the lungs and the stomach, or to the pneumogastric or vagus nerve.

Portal ( $\left.p \bar{o} r^{\prime}-t a l\right)$. Pertaining to the porta (gate) or hilum of an organ, especially of the liver, as the portal vein.

Postcaval (pōst-k $k \bar{\alpha}^{\prime}-v a l$ ). Pertaining to the postcava; the postcaval vein, formerly called the inferior vena cava, or vena cava ascendens.

Precaval ( $\left.p r \bar{e}-k \bar{a}^{\prime}-v a l\right)$. Pertaining to the precava; the anterior caval vein, formerly called the superior vena cava, or vena cava descendens.

Pronation ( $\left.p r \bar{o}-n \bar{\alpha}^{\prime}-s h u n\right)$. The turning of the palm downward.

Protoplasm ( $\left.p r \bar{o}^{\prime}-t \bar{o}-p l a z m\right)$. An albuminous substance, ordinarily resembling the white of an egg, consisting of carbon, oxygen, nitrogen, and hydrogen in extremely complex and unstable molecular combination, and capable, under proper conditions, of manifesting certain vital phenomena, such as spontaneous motion, sensation, assimilation, and reproduction, thus constituting the physical basis of life of all plants and animals.

Ptyalin $\left(t \bar{t}^{\prime}-a-l i n\right)$. An amylolytic or diastasic ferment found in saliva, having the property of converting starch into dextrin and sugar.

Pulmonary ( $\left.p u l^{\prime}-m \bar{o}-n a-r i\right)$. Pertaining to the lungs.

Pylorus $\left(p \bar{i}-l \bar{o}^{\prime}-r u s\right)$. The opening of the stomach into the duodenum.

Radius ( $\left.r \bar{a}^{\prime}-d i-u s\right)$. The outer of the bones of the forearm.

Renal ( $\left.r \bar{e}^{\prime}-n a l\right)$. Pertaining to the kidneys.

Rennin (ren'-in). An enzyme, or ferment, to whose action is due the curdling or clotting of milk produced upon the addition of rennet.

Retina (ret'-i-nä). The chief and essential peripheral organ of vision; the third or internal coat or membrane of the eye, made up of the end organs or expansion of the optic nerve within the globe. 
Sacrum ( $\left.s \bar{a}^{\prime}-k r u m\right)$. A curved triangular bone, composed of five consolidated vertebrae, wedged between the two iliac (pelvic) bones, and forming the dorsal boundary of the pelvis.

Scapula $\left(s k a p^{\prime}-\bar{u}-l \ddot{a}\right)$. The shoulder-blade.

Sciatic $\left(s \bar{i}-a t^{\prime}-i k\right)$. Pertaining to the ischium; the sciatic nerve, the main nerve of the thigh.

Sclerotic (skle-rot'-ik). Hard, indurated; pertaining to the outer coat of the eye.

Semilunar (sem-i-lü'-när). Resembling a half-moon in shape; semilunar valves, pocket-like valves at the beginning of the aorta and pulmonary artery.

Serous (s $\bar{e}^{\prime}-$ rus $)$. Pertaining to, characterized by, or having the nature of, serum.

Serum (sé'-rum). The yellowish fluid separating from the blood after the coagulation of the fibrin.

Solar plexus ( $\left.s \bar{o}^{\prime}-l a ̈ r\right)$. Solar, with radiations resembling the sun.

Sphincter (sfing $k^{\prime}$-ter). A muscle surrounding and closing an orifice.

Splenic (splen'-ik). Pertaining to the spleen.

Steapsin (ste $\left.p^{\prime}-\sin \right)$. A diastasic ferment which causes fats to combine with an additional molecule of water and then split into glycerine and their corresponding acids.

Sternum (ster'-num). The breast-bone.

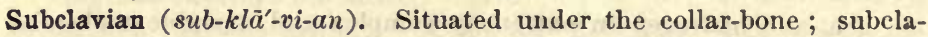
vian artery and vein.

Sublingual (sub-ling'-gwal). Lying beneath the tongue, as sublingual gland.

Submaxillary ( $\left.s u b-m a k^{\prime}-s i-l a-r i\right)$. Lying beneath the lower maxilla, as submaxillary salivary gland.

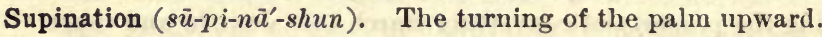

Synovia $\left(s \bar{i}-n o^{\prime}-v i-\ddot{a}\right)$. The lubricating liquid secreted by the synovial membranes in the joints.

Tarsus (tär'-sus). The instep, consisting of seven bones.

Temporal (tem'-pō-ral). Pertaining to the temples, as temporal artery, vein, muscle, etc.

Tetanus (tet'-a-nus). A spasmodic and continuous contraction of the muscles, causing rigidity of the parts to which they are attached.

Thein $\left(t h \bar{e}^{\prime}-i n\right)$. An alkaloid found in tea.

Theobromin (the-ō-bró-min). A feeble alkaloid obtained from cacaobutter; the essential substance found in cocoa and chocolate. 
Thyroid (thi'́-roid). Shield-shaped, as the thyroid cartilage of the larynx.

Tibia $\left(t i b^{\prime}-i-\ddot{a}\right)$. The larger (inner) of the two bones of the le g, commonly called the shinbone.

Trachea ( $\operatorname{tr} \bar{a}-k \bar{e}^{\prime}-a$ or $\left.\operatorname{tr} \bar{a}^{\prime}-k e-a\right)$. The windpipe.

Triceps (trï'-seps). Triceps of the arm, the extensor of the arm, lying along the back of the humerus.

Tricuspid (tri-kus'-pid). Having three cusps or points, as the tricuspid valve.

Trypsin $\left(\right.$ trip $\left.^{\prime}-\sin \right)$. The proteolytic ferment of pancreatic juice.

Ulna $\left(u l^{\prime}-n \ddot{a}\right)$. The larger (inner) of the two bones of the forearm.

Ureter $\left(\bar{u}-r \bar{e}^{\prime}-t e r\right)$. The tube conveying the urine from the pelvis of the kidney to the bladder.

Vaso-constrictor (vas'-j-kon-strik'-tor). Causing a constriction of the blood-vessels.

Vaso-dilato ${ }^{-}\left(v a s^{\prime}-\bar{o}-d i-l \bar{a}^{\prime}-t o r\right)$. Pertaining to the positive dilating motility of the non-striated muscles of the vascular system.

Vaso-motor (vas-ō-mo'-tor). Serving to regulate the tension of the blood-vessels, as vaso-motor nerves; including vaso-dilator and vaso-constrictor mechanisms.

Ventricle (ven'-tri-kl). Applied to certain structures having a bellied appearance. The cavities of the heart from which the blood is forced out through the arteries.

Vesicle $\left(v e s^{\prime}-i-k l\right)$. A small, membranous, bladder-like formation, as air vesicle.

Villus (vil'-us), pl. villi. One of the numerous minute vascular projections from the mucous membrane lining the small intestine, for absorbing digested food.

Vitreous (vit'-re-us). Glass-like, as the clear, jelly-like, vitreous humor of the eye. 


\section{N D E X.}

Abdominal aorta, 69 .

Muscles, 195.

Organs, 195.

Viscera, weight of, 386.

Absorption, 219.

Checking, 232.

Of fats, 222.

Plan of, 220.

From the stomach, 215.

Accessory foods, 167.

Accidents, 328.

Acetic acid, 338 .

Acids, 298, 338.

Acetic, 338.

Of the body, 385 .

Carbolic, 338.

Carbonie, 341.

Citrie, 338.

Hydrochloric, 207.

Medicines, 202.

Muriatic, 338.

Nitric, 338.

Oxalie, 338.

Prussie, 338.

Sulphurie, 338.

Tartarie, 338.

Aconite, 340.

Acro-nareoties, 340 .

Action of auriele, 73 .

Heart, 72.

Heart of frog, 72 .

Large arteries, 76.

Medium-sized arteries, 76.

Saliva, 205.
Action of salivary glands, 204.

Valves of the heart, 73,74 .

Adam's Apple, 320, 321.

Adjustment of lens for distance, 308 .

Afferent root of spinal nerve, 40.

After-image, negative, 312 .

Positive, 312.

After-pressure, 288.

Air, complemental, 117.

Composition of, 122.

Currents around stove, 140 .

Effect of rain on, 143.

Effect of snowstorm, 143 .

Reserve, 118.

Residual, 119.

Tidal, 117.

Vesicles, 105, 112, 129.

Aladdin oven, 371,374 .

Albinos, 152.

Albumen, 168 .

Albuminuria, 245.

Aleohol, 175, 340 .

Affinity for water, 177 .

Binz, 186.

Brunton, 274.

And erime, 176, 178.

Cumulative effects of, 175 .

Effect on eireulation, 93.

Effeets on nervous system, 274 .

And energy, 176.

In the exeretions, $180,190$.

And exposure to cold, 177 .

Gaule, 182.

Greely, 184. 
Alcohol habit, 176.

Halliburton, 189.

As a heat producer, 176 .

Helmholtz, 182.

Hodge, 182.

Howell, 180.

Jenkins, 178.

And longevity, Baer, 188.

Lusk, 179.

M'Kendrick, 190.

And mountain climbing, 181.

Oxidation of, in the body, 176,180.

And poverty, 176.

Rohé, 186.

Smith, 182.

Snell, 181.

Stanley, 184.

In the stomach, 179.

Thompson, 182.

And training, 177.

Woodhull, 184.

Woodruff, 185.

Alkalies, 338.

Of the body, 385 .

Alveoli, 105.

Ammonia, 300, 338.

Inhalation of, 330,341 .

Amount of digestive liquids, 385 .

Urine, 166.

Amplitude of respiration, 299.

Amylopsin, 207, 218.

Anabolic stair, 248.

Anabolism, 250.

Anatomy, definition of, 5 .

Anesthetics, 278.

Animal heat, restoration of, 333 .

Irritants, 340 .

Animals, cold-blooded, 160.

Warm-blooded, 160 .

Anterior articulating process, 349 .

Antidotes to poisons, 336, 338-341.

Antimony, 339.

Antiseptics, 377.

Anvil, 317.
Aorta, 56.

Abdominal, 69.

Thoracic, 69.

A pes, brain centers of, 261.

A pex of heart, 57, 75.

Lung, 56, 108.

A poplexy, 266.

Apparatus, injecting, 68 .

Appendicitis, 228.

Appendicular skeleton, 346.

Appendix vermiformis, 228 .

Aqueous humor, 303.

Arbor vitæ, 256.

Arch of aorta, 69.

Neural, 346.

Argon, 122.

Aristotle's experiment, 273.

Arsenic, 339.

Arteries, 54, 70, 71.

Action of large, 76 .

Action of medium-sized, 76.

Bleeding from, 328.

Cardiac, 61.

Carotid, 69, 71.

Carotid, bleeding from, 328 .

Coats of, 78.

Coronary, 61.

Distribution of, 69, 71 .

Femoral, bleeding from, 328.

Gastric, 69, 71.

Hepatic, 69, 71, 217.

Iliac, 70, 71 .

Injection of, 65 .

Mesenteric, 70, 217.

Muscle fiber in, 77, 78.

Pancreatic, 71.

Pulmonary, 58.

Renal, 70, 71, 162.

Splenic, 69, 71 .

Structure of, 78, 79.

Subclavian, 69, 71 .

Articulating processes, 348, 349 .

A rtificial butter, 357 .

Arytenoid cartilage, 322 . 
Arytenoid muscle, 323.

Ascending colon, 228.

Asiatic cholera, 145.

Assimilation, 249.

Association fibers, 269, 270.

Atkinson, Aladdin oven, 372, 374.

Atlas, 350.

Auditory meatus, 317.

Nerves, 255, 259.

Auricle, 57.

Auricles, action of, 73 .

Contraction of, 72 .

Automaton, 260.

Axial skeleton, 346.

Axis, 350.

Axis-cylinder, 34 .

Bacilli, 146.

Bacillus tuberculosis, 145.

Bacon, 370.

Bacteria, 146.

Of disease, 146.

Of milk, 149.

Of putrefaction, 149.

Baer, alcohol and longevity, 188.

Baking-powder, 229.

Bananas, 229.

Baryta, 338.

Base of lung, 108.

Baseball, 237.

Bath mits, 233. .

Bathing, 233.

The sick, 343.

Baths, cold, 233.

Prolonged, warm, 234.

Bayard Taylor, hashish, 280.

Beans, 169, 230.

Digestibility of, 360 .

Bear and candle, 247.

Beat of heart, 75 .

Of frog, 72 .

Bee-stings, 330.

Beef, 168.

Extracts, 175.
Beef tea, 175.

Beefsteak, composition of, 226.

Beer, root, 179.

Beets, 230.

Belladonna, 278, 340.

Biceps muscle, 10.

Bicuspid teeth, 200.

Bicycling, 236.

Bile, 217.

Duct, 196.

Functions of, 218.

Sac, 196.

Bills of fare, $367,375,376$.

Binz, on alcohol, 186.

Bismuth, 339.

Bites of cats and dogs, 337 .

From snakes, 337 .

Bitters, 298.

Bittersweet, 341.

Blackberries, 230.

Bladder, 161, 195.

Muscle fibers of, 76.

Blaikie, How to Get Strong, 28, 235.

Sound Bodies, 28.

Bleeding from arteries, 328.

Femoral artery, 328.

Lungs, 329.

The nose, 329 .

Stomach, 329.

Veins, 329.

Blind spot, 310 .

Blindness, color, 311.

Blister, 152.

Blood, the, 94.

Amount of, 97.

Blister, 152.

Coagulation of, 97 .

Color of, 131.

Composition of, 384 .

Corpuscles, 94, 95.

Distribution of, 97.

Frog's, 51.

Gases of, 130.

Poisoning, 145. 
Blood, transfusion of, 103.

Ventilation of, 136.

Work of, 48.

Blood-flow, in capillaries, 78.

Experiments with, 79.

Rate of, 84 .

Blood-supply, of brain, 263, 265.

Regulation of, $76,87$.

Blue vitriol, 206, 339 .

Blushing, 86.

Boats upsetting, 335 .

Bodily Exercise, Lagrange, 239.

Body cavities, 382 .

Body, ledger account of, 241.

And locomotive, 134.

And stove, 134.

Tissues of, 386.

Boiled milk, 230.

Boiling meat, 193.

Water, 172.

Bone, composition of, 354 .

Forceps, 33, 301.

Gross structure of, 23, 24.

Hyoid, 322.

Microscopic structure of, 352.

Study of long, 23.

Bones, broken, 330 .

Of the ear, 317.

Functions of, 27.

Hygiene of, 355.

As levers, 26.

Table of, 380 .

Turbinated, 299.

Bouillon, 175.

Bowels, cold in, 233.

Boxing, 238.

Brackett, Technique of Rest, 281.

Brain, blood-supply of, $263,265$.

Of cat or rabbit, 253.

Functions, location of, 261.

Ganglia of, 258.

Preservation of, 253, 387.

Rest, 263.

And spinal cord, 29.
Brain, weight of, 386 .

Work, 263.

Workers, 264.

Brainless dog, 260.

Frog, 259.

Pigeon, 259.

Bran, 170.

Bread, composition of, 226.

Entire wheat, 170.

Hot, 229.

Whitê, 229.

Breathing, hygiene of, 120.

Through the mouth, 142.

Broken bones, 330 .

Bronchi, 57, 105.

Brunton, on alcohol, 274.

Buds, taste, 298.

Bulb, spinal, 33, 254, 263.

Burns, 329.

Butter, artiflcial, 357.

Composition of, 226.

Cabbage, raw, 230.

Caffein, 175.

Cake, 229.

Calcium carbonate, 173.

Phosphate, 173.

Calf, larnyx of, 321.

Muscle of frog, 13, 14.

Caliber of arteries, 78, 87, 88.

Call, Power through-Repose, 47, 281.

Camel's hump, 246.

Camphor, 340.

Canal, central, 254.

Haversian, 352.

Semicircular, 318.

Canaliculi, 353.

Candle and bear, 247.

Burning, 125.

And energy, 251.

Cane sugar, 219.

Canine teeth, 200.

Capacity of the lungs, 117 .

Capillaries, 54. 
Capillaries, blood-flow in, 78 .

Of lungs, 112.

Lymph, 98.

Pulmonary, 105.

Structure of, 79.

Capsule of lens, 304.

Urinary, 165.

Carbohydrates, 170, 359.

Carbohydrate foods, 170, 359 .

Carbolic acid, 338.

Carbon dioxid, in the air, 122.

In air and breath, 127.

Generation of, 124 .

Test of, 125.

In violent exercise, 135 .

Carbonate of calcium, 173.

Carbonic acid, 341.

Carbonic oxid, 341.

Care of the ear, 318.

Of the eyes, 312.

Of the sick, $342,343$.

Of the teeth, 202 .

Carnivora, 174.

Carotid artery, 69.

Bleeding from, 328.

Carpets, objections to, 147.

Cartilage, 25.

Arytenoid, 322.

Cricoid, 322.

Intervertebral, 350 .

Thyroid, 321.

Of windpipe, 55, 107.

Casein, 167, 168.

Cataract, 305.

Cats, bites from, 337 .

Cauliflower, 230.

Caustic potash, 206.

Cavities of the body, 382 .

Serous, 102.

Cavity, marrow, 24.

Medullary, 23.

Neural, 31.

Peritoneal, 102.

Cecum, 196, 228.
Celery, 230.

Cell, 6.

Cells, ciliated, 107.

Size of, 387.

Cellulose, 359 .

Cement of tooth, 200.

Center, for hearing, 262.

For respiration, 122.

For smell, 262.

For speech, 262.

For vision, 262.

Of distinct vision, 306 .

Centers, motor, 261.

Nerve, of glands, 155.

For sensation, 262.

Central canal, 254.

Fissure, 262.

Centrum, 346.

Cereals, 169.

Cerebellum, 33, 256.

Functions of, 263.

Cerebral cortex, 261.

Cerebrum, 33, 255, 256.

Functions of, 259.

Cervical vertebræ, 349 .

Cesspools, 171, 172.

Chain, sympathetic nerve, 90 .

Changing underwear, 161.

Cheese, 169, 229, 370.

For dessert, 231.

Making, 214.

Chemical composition of bone. 354 .

Chemistry of cookery, 372.

Of respiration, 123.

Chewing gum, 205.

Chloral, 340.

Chlorine, 341.

Chloroform, 341.

Chlorophyll, 251.

Chocolate, 175, 229.

Cholera, Asiatic, 145.

Chordæ tendineæ, 60, 73.

Choroid coat, 304.

Chyle, 223. 
Chyle, reveptacle of, 98, 223.

Chyme, 215.

Composition of, 216.

Cider, 179.

Fermentation of, 145.

Cilia, 107.

Ciliary muscle, 307,309 .

Processes, 304.

Circulation of blood, 48 .

Of blood, effect of alcohol on, 93 .

Of lymph, 100.

Plan of, 85, 131, 242, 243, 244.

Portal, 217.

Rate of, 381.

Circulating proteid, 249.

Circumvallate papillæ, 297.

Cistern, 171.

Citric acid, 338.

Classification of joints, 355 .

Clot, blood, 97.

Clothes, effect of, 158.

Clothing, 160.

Taking fire, 330 .

Clum, Inebriety, 275.

Coagulation of blood, 97.

Coagulum, 97.

Coat, ehoroid, 304.

Sclerotic, 304.

Coats of artery, 78.

Of stomach, 213.

Coceyx, 350.

Cochlea, 317.

Cocoa, 175, 229.

Codfish, 169.

Coffee, 173, 175, 230.

Toast, 230.

Wheat, 230.

Colchicum, 340.

Cold bath, 233.

Blooded animals, 160 .

In the bowels, 233.

Spots, 291.

Taking, 232.

Colocynth, 340.
Colon, ascending, 228.

Descending, 228.

Transverse, 228.

Color blindness, 311.

Of the blood, 131.

Sensations, 311 .

Colorado, 161.

Combustion, 135.

Common sensations, 284.

Complementary foods, 173.

Composition of air, 122.

Of beefsteak, 226 .

Of blood, 384 .

Of bone, 354 .

Of bread, 226.

Of butter, 226.

Of foods, 384 .

Of gastric juice, 385 .

Of lymph, 101.

Of milk, 226.

Of potatoes, 226 .

Of sweat, 156.

Of urine, 162.

Compound glands, 224.

Conduction of heat from skin, 158 .

Cone, urinary, 164.

Cones and rods of eye, 305,306 .

Conium, 340.

Conjunctiva, 301.

Consciousness, 261.

Conservation of energy, 252.

Constipation, 228.

Constipating foods, 229.

Constrictors, 92 .

Consumption, 145, 146.

Prevention of, 148.

Contagious diseases, 145 .

Of the eye, 315 .

Contraction of auricle, 72,73 .

Of ventricle, 72,73 .

Control of respiration, 122.

Convalescence and eyes, 314 .

Convection, 140.

Of heat from skin, 158. 
Convolutions of the brain, 257.

Of cerebrum, 33 .

Cookery, Chemistry of, Williams, 372.

Cooking, 192.

Coördination, 263.

Copper, 339.

Sulphate, 206.

Copperas, 339.

Cord, spinal, 88.

Cords, vocal, $322,324,325$.

Corn, 230.

Cornea, 301.

Corner stone of physical science, 252.

Coronary artery, 61 .

Vein, 60.

Corpus arantii, 62.

Corpuscles of blood, 51, 95 .

Colorless, work of, 148.

Red, work of, 128.

Of touch, 286.

Correlation of energy, 252.

Corrosive sublimate, 339 .

Cortex, cerebral, 261.

Cotton in the ears, 318.

Coughing, 116.

Cracked wheat, 229.

Cramps, 42. ,

Cranial nerves, 254, 260 .

Cricoid cartilage, 322 .

Crico-arytenoid muscle, lateral, 323.

Posterior, 322.

Crossing of fibers of optic nerves, 311.

Crothers, on alcohol, 275.

Croton oil, 340.

Crown of tooth, 199.

Crying, 116.

Crystalline lens, 303.

Cultivation of the voice, 327 .

Current, induction, 272.

Interrupted, 272.

Custards, 229.

Cutaneous sensations, 285 .

Cutter, on alcohol, 94.
Daily excretions, 381 .

Expenditure of the body, 387 .

Income of the body, 387 .

Ration of a United States soldier, 383 .

Dancing, 239.

Dandruff, 152.

Dead dust, 143.

Death from breaking neck, 122 .

Decay of teeth, 202.

Deep breathing, 121.

Dejecta, 172.

Dental formula, 200.

Dentine, 200.

Dentist, 202.

Dermis, 151.

Descending colon, 228.

Dessert, 231.

Deterioration, moral, from alcohol, Martin, 277.

Dextrose, 219.

Diabetes, 245.

Dialysis, 221.

Diaphragm, 108, 196.

Ventral view, 106.

Diarrhea from cold, 232.

Effect on strength, 232, 385 .

Effect on urine, 166.

Diet in cold climates, 174.

Necessity of mixed, 173.

Diffusion, 139.

Of liquids, 221.

Digastric muscles, 203.

Digestion, hygiene of, 230.

Organs of, 198.

Outline of, 227.

Time of, 215, 386 .

Digestive liquids, amount of, 385 .

Tube, as a whole, 224.

Digitalis, 340 .

Dinner, a good, 231.

Dioxid, carbon, 125.

Diphtheria, 145.

Direct heating, 141. 
Disease of the eyes, contagious, 315 . Germs, 145.

Diseases, contagious, 145.

Of Inebriety, 275 .

Disinfectants, 377.

Disinfection, 377.

Dislocation, 355.

Dissection of brain, 253.

Of circulatory system, 69.

Of digestive system, 195.

Of eye, 302 .

Of frog, 37.

Of head of rabbit, 202.

Of heart, 55.

Of kidney, 162.

Of larynx, 321.

Of lungs, 59.

Of muscle, 12, 13.

Of nervous system, 29.

Distinct vision, center of, 306 .

Distribution of arteries, 69, 71 .

Of veins, 69, 71 .

Dodging, 42.

Dog, bite of, 337 .

Brainless, 260.

Donoghue, on pie, 231.

Dreams, 271.

Dried fruits, 361.

Meats, 229.

Drinks, hot, 232.

Drinking cold water, 173.

Water, 172.

Drinks, temperance, 179.

Dropsy, 102.

Drowning, resuscitation from, 331.

Drum-skin, 317.

Duct, bile, 196.

Of lachrymal gland, 307.

Lymph, 98.

Pancreatic, 196.

Of salivary glands, 203, 204.

Of sweat gland, 154, 155.

Thoracic, 98, 222.

Ducts, of glands, 224.
Dulcamara, 341.

Duodenum, 196.

Dura mater, 33, 253.

Duration of impressions of light, 312

Dust, dead, 143.

Of house, 147.

Live, 144.

Sources, 143, 144.

Ear, bones of, 317 .

Care of, 318.

External, 316.

Internal, 316.

Middle, 316.

Economy in buying meats, 370 .

Household, scope of, 364 .

Eddy, a living, 245.

Efferent root of spinal nerve, 40.

Eggs, 169, 370.

Electric light for reading, 315 .

Electrical stimnli, 267.

Electricity and nerve impulse, 44.

Elements of the body, 383 .

Emetics, 336.

Emulsion, 208, 219.

Enamel, 200.

Endothelium, 77, 78 .

Energy, conservation of, 252.

Correlation of, 252.

Dormant in nuscle, 136.

Liberation of, 251.

Loss of, 251.

Potential, 115.

Solar, 251.

Source of, 248.

And starvation, 251.

Storing of, 251.

English women as walkers, 237.

Enlargement, cervical, 32.

Lumbar, 32.

Entire wheat, 229.

Flour, 170.

Enzymes, 206.

Epidermis, 151. 
Epidermis, structure of, 153.

Epiglottis, 210, 321.

Epithelial cells, 6.

Equilibrium sense, 317.

Ergot, 341.

Erysipelas, 145.

Esophagus, 55, 196.

Ether, inhalation of, 341.

Eustachian tube, 210, 317.

Evaporation, cooling effect of, 158 .

Excretion, daily, 381.

Need of, 150.

Exercise, 4, 234.

Bodily, Lagrange, 239.

Effect of, 159.

Forms of, 236.

Physiology of, Lagrange, 28.

Expectoration, 146.

Expenditure of the body daily, 387 .

Experiment, Aristotle's, 273.

Experiments with pulse, 81 .

Illustrating movements of respiration, 109.

Illustrating chemistry of respiration, 123.

Expiration, 114, 116.

Extensor muscle, 10, 12.

External ear, 316.

Parts of the eye, 301

Extract of beef, 175 .

Eye, dissection of, 302 .

External parts of, 301.

Protection of, 307.

Regulation of amount of light admitted, 311.

Eyes, care of, 312.

Contagious disease of, 315 .

During convalescence, 314 .

Eyeball, muscles of, 301 .

Eyelids, 307.

Facets, 350 .

Facial nerves, 255, 259.

Fainting, 265, 330.
Fare, bills of, $367,375,376$.

Farina, 230.

Fasting, Tanner, 381.

Fat and starvation, 247.

As storage tissue, 246.

Weight of, in body, 386 .

Fats, as food, 169.

Importance of, 357.

Kinds of, 357.

Melting points of, 357 .

Fatigue, 264, 265.

Fehling, test tablets, 206.

Femoral artery, bleeding from, 328 .

Fermentation of cider, 145.

Ferment, salivary, 205.

Ferments, unorganized, 206.

Fever, during a cold, 232.

Scarlet (see Scarlatiua).

Typhoid, 145, 172.

Yellow, 145.

Fiber, plain muscle, 76, 77 .

Fibers, association, 269, 270.

Of optic nerve, crossing, 311.

Fibrin, 97, 168.

Filiform papillæ, 297 .

Filtration, 221.

Fire, of clothing, 330 .

Fireplaces, 139.

Fish, 169.

Poisonous, 340.

Salted, 229.

Fishing, 237.

Fissure, central, 262.

Of Silvius, 262.

Flavors, 298, 362.

Flexor, 10, 12.

Flexure, sigmoid, 228.

Flour, entire wheat, 170.

Graham, 229.

White, 170.

Fluids of the body, 285.

Food, 167.

Preservation of, 149.

Foods, accessory, 167. 
Foods, carbohydrate-containing, 170.

Complementary, 173.

Composition of, 384.

Constipating, 229.

Time of digestion, 386 .

Fatty, 174.

Laxative, 229.

Proteid-containing, 168.

Foodstuffs, 167.

Football, 237.

Force, indestructibility of, 251.

Forces of inspiration, 115.

Formalin, 253.

Formol, 253.

Forms of exercise, 236.

Formula, dental, 200.

Foster, on alcohol, 93.

Foul-air shaft, 141.

Fowler's Solution, 339.

Foxglove, 340.

Freckles, 152.

Frog, brainless, 259.

Heart, 72.

How to kill, 13.

Web of, circulation in, 52, 53 .

Fruit, best time for, 232.

Fruits, 361.

Dried, 229.

Frying, 193.

Function, definition of, 5 .

Functions of cerebellum, 263.

Of cerebrum, 259.

Of nerve roots, 40 .

Of skin, 157.

Gungiform papillæ, 297.

Furnaces, 140.

Game, wild, 230.

Ganglia, 36.

Of the brain, 258.

Sympathetic, 88, 89, 91.

Ganglion, 32.

Gargling, 117.

Gases of the blood, 130.
Gases, poisonous, 341 .

Gastric glands, 213.

Juice, 213.

Juice, action of, 207.

Juice, composition of, 385 .

Gaule, on alcohol, 182.

Gelatin, 168.

General sensations, 267.

Germs, disease, 145.

Yeast, 145.

Gland, essential features of, 155.

Lachrymal, 307.

Oil, 151.

Parotid, salivary, 202.

Salivary, diagram of, 203.

Sub-lingual, salivary, 204.

Sub-maxillary, salivary, 203.

Sweat, 151.

Glands, compound, 224.

Evolution of, 155.

Excretory, 156.

Gastric, 213.

Intestinal, 216.

Lymphatic, 57, 99, 222.

Oil, 156.

Salivary, action of, 204.

Simple, 156, 224.

Sweat, 154.

Sweat, number of, 381 .

Glossary, 389.

Glosso-pharyngeal nerve, 255, 259.

Gluten, 168.

Glycerine extract of pancreas, 208.

Glycogen, 218, 247.

Graham flour, 229.

Grains, the, 170.

Granula, 229.

Grape sugar, 206, 219.

Grates as ventilators, 138.

Gray matter, activity of, 266 .

Matter of spinal cord, 34.

Nerve fibers, 34.

Greely, on alcohol, 184.

Green peas, 230. 
Growth of hair, 154 .

Of nail, 154.

Gullet, 55, 196, 321.

Gum chewing, 205.

Gustatory nerve, 258, 298.

Habits, 281.

Hair, 151.

Growth of, 154.

On head, number, 381.

Halliburton, on alcohol, 189.

Hallucinations, 270.

Hammer, 317.

Hard water, 171.

Palate, 210.

Harmony in muscle action, 45 .

Hashish, 270, 280.

Hauser, Kaspar, 284.

Haversian canals, 352.

Hawking, 117.

Head, number of hairs, 381.

Of rabbit, dissection, 202.

Hearing, center, 262.

Sense of, 316.

Heart, action of, 62, 72.

And lungs, 55.

Apex of, 75.

Beat, rate of, $49,72,75$.

Dissection of, 59 .

Frog's, 72.

Muscle, 90.

Nourishment of, 86 .

Palpitation of, 175.

Sounds of, 75 .

Structure of, 59.

Valves of, $60,61,62,74$

Weight of, 386 .

Work and rest of, 75 .

Heat, distribution of, 159 .

Production of, 132.

Production, regulation of, 159 .

Radiant, 140.

Regulation by skin, 157 .

Restoration of, 333 .
Heat saver, the, 367.

Heating, direct, 141.

Indirect, 141.

And ventilation, 137.

Hellebore, 341.

Helmholtz, on alcchol, 182.

Hemiglobin, 96, 128.

Hemiplegia, 261.

Hemispheres of cerebrum, 33, 254, 261.

Hemlock, 340.

Hemorrhage, how to stop, 328 .

Of lungs, 329.

Of stomach, 329 .

Hepatic artery, 217.

Vein, 70.

Herbivora, 174, 216.

Hibernating animals, 247.

Hiccuping, 117.

Hilum, 161, 162.

Hodge, on alcohol, 182.

Hopping, 236.

Horn, ventral, of gray matter of spinal cord, 35.

Horseback riding, 236, 237, 238.

Hot bread, 229.

Hot drink, 232.

House dust, 147.

Howell on absorption, 221.

Humor, aqueous, 303.

Vitreous, 304.

Hunger, 267, 296.

Huxley, living eddy, 245.

Hydrochloric acid, 207.

Hydrogen, 169.

Sulphuretted, 341.

Hygiene of bones, 355 .

Of breathing, 120.

Definition of, 5 .

Of digestion, 230.

Of joints, 355 .

Hyoid bone, 322 .

Hyoscyamus, 278, 341.

Hypoglossal nerve, 256. 
Ice, artificial, 172.

Impurities of, 172.

Water, $172,173$.

Iliac arteries, 70.

Veins, 70.

Illusions of touch, 290.

Image inverted on retina, 308 .

Imagination, 273.

Importance of fats, 357 .

Impressions of light, duration of, 312.

Impurities in water, 171.

Incisor teeth, 199, 200.

Income of body, daily, 387 .

Indestructibility of force, 251 .

Of matter, 250.

Indirect heating, 141.

Induction current, 272.

Inebriety, Clum, 275.

Diseases of, Crothers, 275.

Inferior oblique muscle, 301.

Inhibition, 44, 92.

In fainting, 265 .

Reflex, 92.

Injecting apparatus, 68.

Arteries, 65.

Injection mass, 66 .

Of thoracic duct, 223.

Innominate veiil, 70

Inorganic world, 248.

Insertion of muscle, 12, 15.

Inspiration, 113, 115.

Intelligence, 260.

Intensity of stimulus, 268.

Internal ear, 316.

Respiration, 136.

Interrupted current, 272.

Intervertebral cartilages, 350.

Intestinal glands, 216 .

Juice, 216, 219.

Intestine, 195, 216.

Large, 196.

Small, 196, 216.

Inventors, 264.
Inverted image on retina, 308.

Invertin, 219.

Iodine, 339 .

Iris, 303.

Action of, 311.

Iron, 173, 339.

Oxid, 125.

Sulfate, 172.

Irritants, animal, 340 .

Metallic, 339.

Vegetable, 340.

Jolnts, classification of, 354 .

Dissection of, 24.

Hygiene of, 355 .

Stiffened, 355.

Judgment, 273.

Jugular vein, 70.

Valves in, 82.

Juice, gastric, 213.

Gastric, composition of, 385 .

Intestinal, 216.

Pancreatic, 217.

Jumping, 236.

Kaspar Hauser, 284.

Katabolic stair, 248.

Katabolism, 250.

Kidney, the, 161.

Diseased, 245.

Dissection of, 162.

Microscopic structure of, 163.

Killing a frog, 13.

A cat, 67 .

Kingsley, The Two Breaths, 136.

Knot, surgeon's, 67 .

Laburnum, 341.

Lachrymal gland, 307.

Lacteals, 220, 222.

Lacunæ, 353.

Lagrange, Physiology of Bodily Fixercise, 28, 239.

Lamellæ, 353. 
Large intestine, 196, 228.

Larynx, 55, 321.

Dissection of, 321 .

Of calf, 321.

Lateral crico-arytenoid muscle, 323.

Laughing, 116.

Laxative foods, 229.

Lead, 339.

Ledger account of body, 241.

Legumin, 168.

Lens capsule, 304.

Crystalline, 303.

Lemons, 229.

Levers, 21.

Liberation of energy, 251.

Ligaments, 24.

Light admitted to eye, regulation of, 311.

Lime, 173, 338.

Liquids of body, specific gravity of, 385.

Digestive, amount of, 385 .

Live dust, 144.

Liver, 195, 217, 218.

As food, 230.

Weight of, 386.

Living machines, 251.

Lobelia, 341.

Lobes, olfactory, 33, 254.

Local sign, 289.

Localization of touch sensations, 288.

Location of brain functions, $261,262$.

Lock-jaw, 42.

Locomotion, 25.

Locomotive and body, 134, 135 .

Longevity and alcohol, Baer, 188.

Loss of energy, 251.

Of water from the body, 383 .

Loudness of voice, 325 .

Lumbar vertebra, 350, 351.

Lung, apex of, 108.

Base of, 108.

Bleeding from, 329 .

Capacity, 117.
Lung diseases, death from, 148.

Dissection of, 55, 59.

Lungs and heart, 55.

Lymph, the, 98, 246.

Capillaries, 98.

Cavities, 98.

Composition of, 101.

Duct, 98.

Around glands, 155.

Spaces, 98.

Veins, 98, 222.

Lymphatics, 222.

Lymphatic glands, 57, 99, 222.

Machines, living, 251.

Magnesium oxid, 125.

Malted milk, 175.

Marrow cavity, 24.

Martin, on alcohol, 277.

Massage, 103.

Masseter muscle, 11, 203.

Master tissue, 252.

Mastication, effects of incomplete, 209.

Mater, dura, 253.

Pia, 253.

Matter, indestructibility of, 250.

M'Kendrick, on alcohol, 190.

Meal, foodstuffs in, 226.

Measles, 145.

Measurement of tissues, 387.

Meat, 168.

Boiling, 193.

Dried, 229.

Economy in, 370.

Smoked, 229.

Meatus, auditory, 317.

Mechanical respiration, 136.

Stimuli, 266.

Mediastinal space, 108.

Medulla oblongata (see Spinal Bulb).

Medullary cavity, 23.

Membrane, mucous, 107, 205, 321.

Tympanic, 317. 
Memory, 273.

Meningitis, 266.

Mercury, 339.

Mesentery, 196, 197.

Mesenteric artery, 70.

Vein, 70.

Metabolism, 250.

Metallic irritants, 339.

Microscopic structure of bone, 352.

Middle ear, 316.

Middlings, 170.

Milk, 167, 169, 370 .

Bacteria in, 149.

Boiled, 230.

Composition of, 226.

Heating, 173.

Malted, 175.

Peptonized, 175.

Teeth, 201.

Mitchell, Wear and Tear, 283.

Mixed diet, necessity of, 173.

Molar teeth, 200.

Molasses, New Orleans, 229.

Mold, 145.

Moral deterioration by alcohol, Martin, 277.

Morphine, 341.

Motion, 9.

Production of, 132.

And sensation, 29.

Motor centers, 261, 262.

Mountain climbing, amount of urea, 248.

Effect of alcohol on, 181.

Mouth, the, 198.

Breathing through, 142.

Movements of respiration, 113.

Mucous glands, 205, 212.

Membrane, 205, 321.

Mucus, 107, 205, 212.

Muriatic acid, 338.

Muscle, action of, 13.

Action of, laws of, 17.

Arytenoid, 323.
Muscle, calf of frog, 13.

Ciliary, 307, 309.

Digastric, 203.

Fiber, non-striated, 77.

Fiber, plain, 76, 77 .

Of heart, 90.

Inferior oblique, 301.

Lateral crico-arytenoid, 323.

Masseter, 203.

And nerve, 12.

Nerve preparation, 13.

Posterior crico-arytenoid, 322.

Sheath, 15.

Striated, 15.

Striped, 15.

Structure of, 14.

Superior oblique, 301.

Temporal, 204.

Thyro-arytenoid, 323.

Muscles, abdominal, 195.

And bones, 20.

Engines of motion, 248.

Experiments with, 10.

Of eyeball, 301.

And Nerves, Rosenthal, 47.

Papillary, 61.

Recti, of the eye, 301 .

Of respiration, 113, 122.

Sphincter, 215.

Of stomach, 213.

Symmetrical development of, 18

Weight of, 386.

Muscular contraction, 17

Sense, 292.

Mustard, 336.

Mutton, 168, 358, 384.

Myosin, 168.

Nails, growth of, 154.

Narcotics, 278, 340.

Nasal passage, 210.

Nature of sensation, 269.

Of sensation, relative, 272 .

Nausea, 284, 294. 
Necessity of mixed diet, 173.

Neck of tooth, 199.

Negative after-image, 312 .

Nerve cells, 35, 36.

Centers, 36, 43, 87, 93, 122, 155, $263,270$.

Centers, of glands, 155 .

Chain, 90, 91.

Current, 38.

Endings in the skin, 285.

Fiber, 33.

Fiber sheath, 34 .

Fibers, vaso-constrictors, 92 .

Fibers, vaso-dilators, 92.

Glosso-pharyngeal, 255, 259.

Impulse, 34, 38.

Optic, 301, 302.

Pneumogastric, 256, 259.

Sciatic, 37.

Stimuli, 266.

Trunk, 40.

Nerves, auditory, 255, 259.

Cranial, 254, 260.

Facial, 255, 259.

Gustatory, 258, 298.

Hypoglossal, 256.

Olfactory, 258, 299.

Optic, 254, 258, 260, 302.

Pneumogastric, 91, 92, 256, 259.

Spinal, 32.

- Structure of, 33.

Of sweat gland, 155.

Sympathetic, 88, 89 .

Vaso-motor, 93.

Neural arch, 346, 348.

Ring, 346, 348.

Neuralgia of face, 254.

Neuroglia, 258.

Newspaper reading, 314 .

Nightshade, 340.

Nitrate of silver, 340 .

Nitric acid, 338.

Nitrogen of air, 122.

Preparation of, 123.
Nitrogen, properties of, 124.

Nitrous oxid, 341.

Nose, bleeding from, 329 .

Nourishment of walls of heart, 86 .

Number of hairs on head, 381 .

Of sweat glands, 381 .

Nurse, the, 343.

Nutrition, 248.

Science of, 365,374 .

Nuts, 231.

Nux vomica, 341 .

Oatmeal, 229.

In drinking-water, 173.

Oblique muscle, inferior, 301.

Superior, 301.

Odontoid process, 350 .

Odors, pungent, 300.

Oil, croton, 340.

Glands, 151, 156.

Savin, 340.

Olfactory lobes, 33, 254.

Nerves, 258, 299.

Onion, 230, 363.

Opium, 279, 341.

Optic nerve, 254, 258, 301, 302.

Crossing of fibers, 311.

Oranges, 229.

Organ, definition of, 5 .

Organs of abdomen, 195.

Of digestion, 198.

Origin of muscle, 12.

Osmosis, 221.

Outline of digestion, 227.

Oven, the Aladdin, 371, 374.

Oxalic acid, 338.

Oxid, carbonic, 341 .

Iron, 125.

Magnesium, 125.

Nitrous, 341.

Phosphoric, 123.

Oxidation of metals, 125.

In the tissues, 132, 134 .

Oxygen in the air, 122 . 
Oxygen, properties of, 124 .

Oxy-hemoglobin, 128.

Oysters, 230.

Pain, 285, 294.

Palate, hard, 210.

Soft, 210.

Palpitation, 175.

Pancreas, 196, 217.

Pancreatic duct, 196.

Juice, 217.

Juice, action of, 207.

Pancreatin, 207.

Papillæ of skin, 151, 153.

Of tongue, 297.

Circumvallate, 297.

Filiform, 297.

Fungiform, 297.

Paralysis, 261.

Paris Green, 339.

Parotid salivary gland, 202.

Parts of the body, weight of, 386 .

Pastry, 229, 232.

Peaches, 229.

Peas, 169, 230.

Pepsin, 213.

Amount of, 213.

Test, 207.

Peptone, 214.

Peptonized milk, 175.

Pericardial fluid, 57.

Pericardium, 57.

Periosteum, 25.

Peristaltic motion, 195.

Peritoneal cavity, 102.

Peritoneum, 195, 197.

Perspiration, amount of, 157.

Insensible, 156.

Sensible, 157.

Variation of, 157.

Dharynx, 210.

Phosphate of calcium, 173.

Phosphates in flour, 170.

Phosphorus, 340, 123.
Phosphoric oxid, 123.

Physical science, corner stone of, 252.

Physiologieal division of labor, 6 .

Physiology, definition of, 5 .

Physostigma, 341.

Pia mater, 253.

Pickpockets, 272.

Pie, 231.

Pigeon, brainless, 259.

Pigment cells, 52.

Pigment layer of eye, 305,307 .

Of skin, 152.

Pike's-Peak, climbing, 181.

Piles, 228.

Pitch of voice, 325.

Plain muscle fiber, 76, 77.

Plan of absorption, 220.

Of circulation, $85,131,242,243$, 244.

Plasma, 52, 95, 97.

Pleura, 56, 108.

Pleurisy, 108, 295.

Plexus, 91.

Brachial, 32.

Solar, 90,91 .

Plums, 229.

Pneumogastric nerves, 91, 256, 259.

Poison ivy, 342.

Poisons and antidotes, 336, 338-341.

Poisoning in food, 149.

Poisonous fish, 3.0 .

Pollen, 144.

Pores, sweat, 151, 154.

Pork, 168, 359, 370.

Portal circulation, 217.

Vein, 70.

Positive after-image, 312 .

Posteaval vein, 70, 217.

Posterior crico-arytenoid muscle, 322.

Potash, 338

Caustic, 206.

Potatoes, 230, 361.

Composition of, 226. 
Potential energy, 115.

Poultry, 229.

Power through Repose, Call, 281.

Precaval vein, 70.

Premolar teeth, 200.

Preservation of brain, 253, 387. Of food, 149.

Pressure sense, 285, 287.

On veins, effect of, 83 .

Privies, 171.

Process, anterior articulating, 348, 349.

Ciliary, 304.

Odontoid, 350.

Posterior articulating, 348,349 .

Spinous, 348.

Transverse, 348.

Of vertebra, $346,348,349$.

Production of heat, 132.

Regulation of, 159.

Pronation, 352.

Protection of eye, 307.

Proteid, circulating, 249.

Proteid-containing foods, 168.

Proteids, 168.

Artificial digestion of, 207, 208.

Characteristics, 168.

Vegetable, 169.

Protoplasm, 6, 249.

Animal and vegetable, 248.

Prunes, 229.

Prussic acid, 338.

Ptyalin, 205.

Pulmonary capillaries, 112.

Pulp cavity of tooth, 199, 200.

Pulse, 49, 81 .

Experiments with, 81 .

Pungent odors, 300 .

Pupil of eye, 303.

Pylorus, 215.

Pyramid, urinary, 163.

Quality of voice, 326 .

Quantity of water in organs, 383.
Rabbit, brain and spinal cord of, 29, 253.

Dissection of head, 202.

Sympathetic nerve of, 86, 92.

Racing, 236.

Radiation of heat from skin, 158.

Rain, effect on air, 143.

Water, 170.

Raspberries, 230.

Rate of blood-flow, 84 .

Of circulation, 381 .

Of heart-beat, 49,72 .

Ration, daily, of U.S. soldier, 383.

Reaction in locomotion, 26.

In expiration, 116.

Time, 268.

Reading, 313.

Receptacle of chyle, 98, 223.

Recreation, 264.

Rectum, 197, 228.

Rectus muscle, 301 .

Reflex action, 36, 38, 268, 269.

Action, essentials of, 41.

Action, importance of, 42

Action of spinal cord of frog, 36 .

Action, process of, 42.

Reflex arc, 38.

Regulation of amount of light to eye, 311.

Of blood-supply, $76,77,87$.

Of heat by skin, 157.

Of heat production, 159.

Reid, bear story, 247.

Relative nature of sensations, 272 .

Renal artery, 70, 162.

Vein, 70, 162.

Rennet, 214.

Rennin, 214.

Repose, Power through, Call, 47, 281.

Respiration, 105.

Abdominal, 116.

Control of, 122. .

Costal, 116.

Effect on circulation, 121. 
Respiration, effect on movement of lymph, 121.

Chemistry of, 123.

Diaphragmatic, 116.

Experiments illustrating, 109.

Hygiene of, 120.

Internal, 136.

Mechanical, 136.

Movements of, 113.

Muscles of, 113, 122.

Rate, how affected, 116.

Thoracic, 116.

Time of, 116.

And work, 133.

Respiratory center, 122 .

Sounds, 121.

Rest and work of heart, 75 .

Technique of, Brackett, 47, 281.

Resting, 281.

The eyes, 314 .

Restoration from fainting, 265,330 .

Of animal heat, 333.

Resuscitation from drowning, 331.

Retina, 305, 306.

Inverted image on, 308 .

Rhubarb, 230.

Rhythmic action of heart, 90 .

Rice, 230.

Ring, neural, 346.

Rods and cones of eye, 306 .

Rohé, on alcohol, 186.

Root beer, 179.

Root of tooth, 199.

Of lung, 108.

Roots, of nerves, dorsal, 32 .

of nerves, ventral, 32.

Rope-skipping, 236.

Rosenthal, Muscles and Nerves, 47.

Round steak, 169.

Rugby, School Days at, 239.

Rugs, advantages of, 147.

Rumford, soup kitchens, 365 .

Running, 26.

Rusty nails, wounds from, 337.
Sac, bile, 196.

Sacrum, 350.

Sago, 230.

Saline solution, normal, 14 .

Salines, 298.

Saliva, action of, 205.

Amount of, 205.

Tests of, 206.

Salivary ferment, 205.

Gland, diagram of, 203.

Gland, parotid, 202, 204.

Gland, sublingual, 204.

Gland, submaxillary, 203, 204

Salts, 173.

Satiety, 284.

Savin oil, 340.

Scarlatina, 145.

Scarlet fever (see Scarlatina).

Scheele's Green, 339.

School Days at Rugby, 239.

Sciatic nerve, 12, 13, 32 .

Sclerotic coat, 304 .

Seasonings, 362 .

Sedgwick and Wilson, 246.

Semicircular canals, 318 .

Sensation, 270.

Centers, 262.

Of color, 311.

And motion, 29.

Nature of, 269.

Sensations, common, 284.

Cutaneous, 285.

General, 267.

Relative nature of, 272.

Of touch, localization of, 288.

Sense of equilibrium, 317 .

Of hearing, 316.

Muscular, 292.

Of pressure, 285, 287.

Of position, 292.

Of sight, 300 .

Of smell, 298.

Of taste, 296.

Of temperature, 290. 
Senses, special, 284.

Sensory root of spinal nerve, 40.

Serous cavities, 102.

Serum, 97.

In blister, 152.

Sewall, on muscular sense, 293.

Sense of touch, 290.

Sheath, medullary, 34 .

Muscle, 15.

Musele fiber, 16.

Nerve fiber, 34 .

Shooting, 237.

Sick, care of, 342.

Sick-room, dust, 344.

Sighing, 117.

Sigmoid flexure, 228.

Sign, local, 289.

Silver, nitrate of, 340 .

Silvius, fissure of, 262.

Simple glands, 156, 224.

Size of cells, 387.

Skeleton, 346, 347, 380.

Appendicular, 346 .

Axial, 346.

Weight of, 352, 386.

Skin, functions of, 157.

As heat regulator, 157.

Nerve endings in, 285.

Structure of, 151.

Thickness of, 152.

Weight of, 152, 386.

Skipping the rope, 236.

Skull, 347, 352.

Sleep, 263, 264.

Loss of, 264.

Sleeplessness, 264.

Sleight-of-hand performer, 272.

Small intestine, 196, 216.

Small-pox, 145.

Smell, brain center of, 262.

Sense of, 298.

Smelling salts, 265.

Smith, on alcohol, 182.

Smoked meats, 229.
Snake bites, 337.

Sneezing, 116.

Prevention of, $345^{\circ}$.

Snell, mountain climbing, 181.

Sniffing, 117.

Snoring, 117.

Snowballing, 236 .

Snowstorm, effect on air, 143.

Soap, 233.

Sobbing, 116.

Soda, 330, 338.

Sodium bicarbonate, 208.

Soft palate, 210.

Solar energy, 251.

Plexus, 90, 91.

Soldier, United States, daily ration of, 383 .

Sound, 317.

Bodies, Blaikie, 28.

Waves, 317.

Sounds of heart, 75 .

Respiratory, 121.

Soups, first course, 231.

Soup-making, 193, 366.

Special senses, 284.

Specific gravity of liquids of body, 385.

Speech, 326.

Center, 262, 270.

Sphincter muscles, 215.

Spices, 363.

Spinach, 230.

Spinal bulb, 33, 254, 263.

Cord, 88.

Cord and brain, cat or rabbit, 29 .

Cord, cross section of, 34 .

Cord, functions of, 43.

Spinous process, 348.

Spitting on floors, 146.

Spleen, the, 102.

Splenic vein, 70.

Spoiling of food, 149 .

Sponge bath, 233.

Spores, 144, 172. 
Spot, blind, 310.

Yellow, 306.

Spots, cold, 291 .

Warm, 291.

Sprains, 355.

Spring water, 171.

Squash, 230.

Stair, anabolic, 248.

Katabolic, 248.

Standing, 25.

Stanley, on alcohol, 184.

Starch, 170, 359.

Excess of, 360 .

Starvation and energy, 251.

And fat, 247.

And weight, 383.

Steak, round, 169.

Tenderloin, 169.

Steapsin, 219.

Stereoscopic vision, 312.

Stevenson and Murphy, on alcohol, 93, 274.

Stiffened joints, 355 .

Stimuli, electrical, 267.

Intensity of, 268.

Of nèrves, 266.

Stirrup, 316, 317.

Stockham, Tokology, 229.

Stomach, 195, 212, 213.

Absorption from; 215.

Bleeding from, 329 .

Digestion, time of, 215.

Muscles of, 213.

Storing energy, 251.

Stove and body, 134.

Stramonium, 278.

Strong, How to Get, Blaikie, 235.

Structure of abdomen, 197.

Arteries, 78.

Bone, 23, 24, 353.

Brain, 253, 255.

Ear, 316.

Eye, 302.

Gland, gastric, 214.
Structure of gland, salivary, 203.

Heart, 59.

Joints, 24.

Kidney, 162.

Larnyx, 321.

Lung, 59, 105, 112.

Muscle, 14.

Nerve, 33.

Nerve fiber, 34 .

Retina, 306.

Skin, 151, 152.

Spinal cord, 35.

Stomach, 213.

Thorax, 106, 108, 110, 114.

Tooth, 199.

Villus, 223, 224.

Strychnine, 341 .

Subclavian artery, 69 .

Vein, 70.

Sublimate, corrosive, 339 .

Sublingual salivary gland, 204.

Submaxillary salivary gland, 203, 204.

Suffocation in wells, 335 .

Sugars, 170, 360 .

Sulphate of copper, 206.

Of iron, 172.

Sulphuretted hydrogen, 341 .

Sulphuric acid, 338.

Summer complaint, 232.

Sunlight, 161.

Sunshine, 161.

Sunstroke, 331.

Superior oblique muscle, 301 .

Rectus muscle, 301 .

Supination, 352.

Swallowing, 210, 212.

Sweat, 151, 156.

Amount of, 156.

Cold, 156.

Composition of, 156 .

Gland, 151, 154.

Glands, number of, 381 .

Pores, 154. 
Sweating, effect on urine, 166.

Sweeping, 147.

In the sick-room, 344.

Swimming, 335.

Symmetry, 18, 19.

Sympathetic nerves, 34, 88, 89, 91 .

Synovia, 24.

Syringe in heart experiments, 65.

In pulse experiment, 80 .

Table of the bones, 380 .

Tag, 236.

Taking cold, 160, 232.

Tanner, fasting, 381 .

Taper, wax, 125.

Tapioca, 230.

Tartar emetic, 339.

Tartaric acid, 338.

Taste buds, 298 .

Sense of, 296.

Taylor, Bayard, hashish, 280.

Tea, 173, 174, 230.

Beef, 175.

Tear, Wear, and, Mitchell, 283.

Tears, 307.

Technique of Rest, Brackett, 47, 281.

Tetanus, 42.

Teeth, the, 199.

Arrangement, 200.

Care of, 202.

Decay of, 202.

Kinds, 200.

Milk, 201.

Nerves of, 254.

Time of appearance, 201.

Temperance drinks, 179.

Temperature affected by clothing, 160.

Affected by food, 160 .

Of body, 157 .

Regulation by skin, 157 .

Of the sick-room, 343 .

Sense, 285, 290.

Temporal muscle, 11, 204.
Tenderloin, 169.

T'endon, Achilles', 12

Tennis, 237.

Test tablets, Fehling's, 206.

Thein, 174.

Theobromin, 175.

Thirst, 267, 296.

Thompson, on alcohol, 182.

Thorax, cross section of, 110 .

Diagrams of, 108, 114.

Shape of, 352.

Ventral view of, 106.

Thoracic aorta, 69.

Duct, 220,222 .

Duct, injection of, 223.

Vertebra, 348.

Viscera, weight of, 386 .

Thyro-arytenoid muscle, 323.

Thyroid cartilage, 321.

Time of gastric digestion, 215, 386 .

Reaction, 268.

Tin, 340.

Tissue, connective, 15, 16.

Tissues, definition of; 5 .

Measurement of, 387.

Of the body, 386.

Oxidation in, 134.

Toadstools, 341 .

Tobacco, 191, 341.

Tokology, Stockham, 229.

Tomatoes, 230.

Tom Brown, 239.

Tongue, the, 198, 297.

Nerves of, 297.

Papillæ of, 297.

Sensitiveness of, 289.

Tooth, crown of, 199.

External parts, 199.

How to make sections of, 200.

Neck of, 199.

Picks, 202.

Powder, 202.

Pulp cavity of, 199, 200.

Root of, 199. 
Tooth, structure of, 200.

Touch corpuscle, 286.

Illusions of, 290.

Sense of, 286.

Touch-sensations, localization of, 288 .

Touching objects, 285.

Trachea, 55, 105.

Transfusion of blood, 103.

Transverse colon, 228.

Process, 348.

Triceps muscle, 10.

Trypsin, 218.

Tube, eustachian, 317.

Tuberculosis, 145.

Turbinated bones, 299.

Turpentine, 341 .

Typhoid-fever, 145, 172.

Tympanic membrane, 317.

Tympanum, membrane, 317.

Underclothing, changing, 161.

Underwear, 161.

Unorganized ferments, 206.

Unstriated muscle fibers, 76, 77.

Upsetting of boats, 335 .

Urea, 161.

Amount of, 248.

Ureter, 161.

Urinary capsule, 165.

Cone, 164.

Pyramid, 163.

Urine, 161.

Amount of, 166.

Composition of, 162.

Valve, mitral, 61.

Tricuspid, 60.

Valves of heart, 60,61 .

Of heart, action of, 73, 74 .

Of lacteals, 223, 224.

Semilunar, 61.

Of thoracic duct, 223.

Of veins, 50, 82, 83.

Vaso-constrictor nerves, 92 .
Vaso-dilator nerves, 92.

Vaso-motor nerves, 93.

Veal, 168.

Vegetable irritants, 340 .

Proteids, 169.

Vegetables, 371.

Vegetarians, 174.

Vein, gastric, 70 .

Innominate, 70 .

Mesenteric, 70.

Pancreatic, 70.

Portal, 70.

Postcaval, 57, 70.

Precaval, 57, 70.

Splenic, 70 .

Veins, 54, 82.

Bleeding from, 329 .

Cardiac, 60.

Coronary, 60 .

Distribution of, 69,71 .

Effect of pressure on, 83 .

Hepatic, 70.

Iliac, 70.

Jugular, 70.

Lymph, 98, 229.

Pulmonary, 58.

Renal, 70, 162.

Structure of, 79.

Subclavian, 70.

Valves of, $50,82,83$.

Ventilation, 137.

of the blood, 136.

Ventricle, 57, 60 .

Contraction of, 72 .

Of brain, 254.

Ventriloquist, 272.

Verdigris, 339.

Vermiform appendix, 228.

Vermilion, 339.

Vertebræ, 346.

Cervical, 349.

Lumbar, 350, 351.

Thoracic, 348.

Vesicles of ling, 105, 112, 129. 
Fiili $, 197,220,223$.

Structure of, 223, 224.

Virchow, on alcohol, 94.

Viscera, abdominal, weight of, 386 .

Thoracic, weight of, 386.

Vision center, 262.

Stereoscopic, 312.

Vital capacity, 118, 119.

Vitreous humor, 304.

Vitriol, blue, 206, 339.

White, 340.

Vocal cords, 322, 324, 325.

Voice, the, 320.

Cultivation of, 327 .

Loudness of, 325 .

In lower animals, 326 .

Quality of, 326.

And speech, 326, 327 .

Volition, 259, 261.

Vomiting, 336.

Walking, 25.

As exercise, 236, 237, 238, 265.

Waller, coasting proteid, 249.

Warm baths, prolonged, 234.

Blooded animals, 160.

Spots, 291.

Water, 170.

Effect of boiling, 172.

Hard, 171.

Loss of from body, 383 .

Rain, 170.

In tissues, 383.

Waste matter, 128, 149, 151, 161, 245.

Organic, 127.

Wear and Tear, Mitchell, 283.

Web, frog's, circulation in, 53, 54.

Weight during starvation, 383.

Of parts of body, 386 .
Wells and cesspools, 171 .

Suffocation in, 335 .

Well-water, 171.

Wheat, 170.

Cracked, 229.

Entire, flour, 170.

Whirlpool and living being, 245.

Whisky for snake-bites, 342 .

Whispering, 327.

In the sick-room, 344 .

White bread, 229.

Flour, 170.

Matter of spinal cord, 34,35 .

Vitriol, 340.

Wild game, 230.

Wilderness cure, 148.

Will, the, 253, 259.

Power, 264.

Williams, Chemistry of Cookery, 372.

Wilson and Sedgwick, 246.

Wind, 139.

Windpipe, 55.

Winking, 42.

Woodhull, on alcohol, 184.

Woodruff, on alcohol, 185.

Woollen clothing, 160.

Work and respiration, 133.

And rest of heart, 75 .

World, inorganic, 248.

Wounds, bleeding from, 328 .

From rusty nails, 337.

From thorns, 337.

Yawning, 117.

Yeast, 145.

Yellow-fever, 145.

Spot, 306.

Zine, 340. 




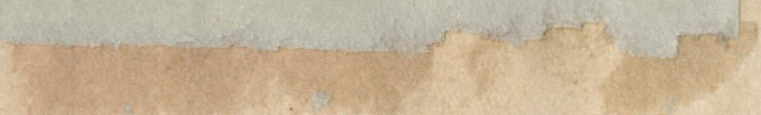

,

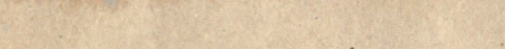




\section{QP36 212412}

C68

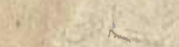

Colton

BIOLOGY WBRRRY

()

3.

UNIVERSITY OF CALIFORNIA LIBRARY

UNIVER

$\ldots+\infty$

$<$

$\therefore, \cdots \cdots$ 
